\title{
Selective, modular probes for thioredoxins enabled by rational tuning of a unique disulfide structure motif
}

Jan G. Felber ${ }^{1}$, Lukas Zeisel ${ }^{1}$, Lena Poczka ${ }^{1}$, Karoline Scholzen², Sander Busker ${ }^{2}$, Martin S. Maier ${ }^{1}$, Ulrike Theisen ${ }^{3}$, Christina Brandstädter ${ }^{4}$, Katja Becker ${ }^{4}$, Elias S. J. Arnér ${ }^{2,5}$, Julia Thorn-Seshold ${ }^{1}$, Oliver Thorn-Seshold ${ }^{1}$

\footnotetext{
${ }^{1}$ Department of Pharmacy, Ludwig Maximilians University Munich, Butenandtstr. 5-13, 81377 Munich, DE.

2 Department of Medical Biochemistry, Karolinska Institutet, Solnavägen 9, 17177 Stockholm, SE.

3 Institute of Pharmacology and Toxicology, Medical Center, University of Rostock, Schillingallee 70, 18057

Rostock, DE.

${ }^{4}$ Interdisciplinary Research Centre (IFZ), Justus Liebig University Giessen, Heinrich-Buff-Ring 26-32, 35392 Giessen, DE.

${ }^{5}$ Department of Selenoprotein Research, National Institute of Oncology, 1122 Budapest, HU.
}

\section{Author Contributions}

J.G.F. performed synthesis, analysis, chemoreductant and enzyme studies, coordinated data assembly and wrote the manuscript. L.Z. performed synthesis, analysis, and chemoreductant assays. L.P. and K.S. performed enzyme titration assays. S.B. and C.B. performed enzyme selectivity screenings. M.S.M. performed synthesis and analysis. U.T. performed early biological evaluations. K.B., E.S.J.A. and J.T.-S. supervised enzymatic selectivity screenings. O.T.-S. designed the concept and experiments, supervised all other experiments, coordinated data assembly and wrote the manuscript, with input from all co-authors.

1. Disulfide-based bioimaging and prodrug approaches..........................................4

2. Fluorescence properties ..............................................................................5

3. Relative kinetics of cyclisation ...................................................................

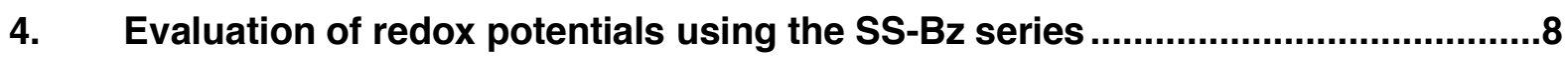

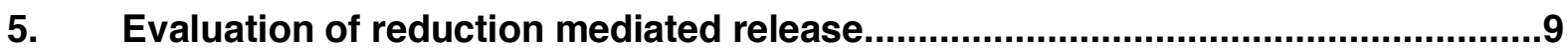

6. Enzyme specifity/activity study ......................................................................14

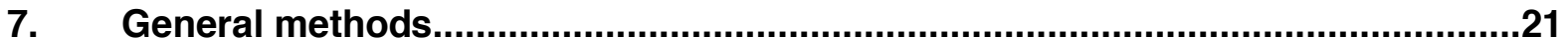

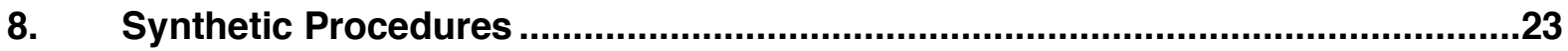

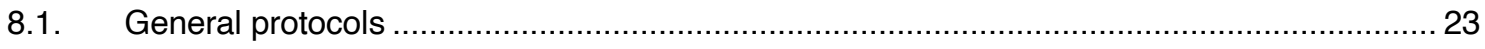

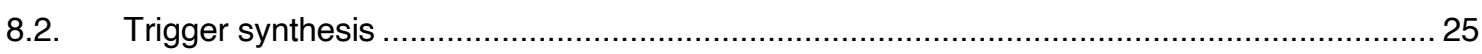

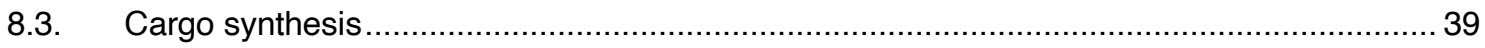

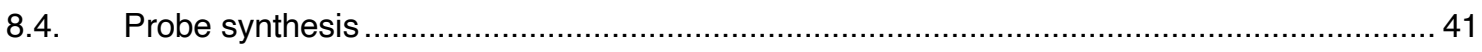

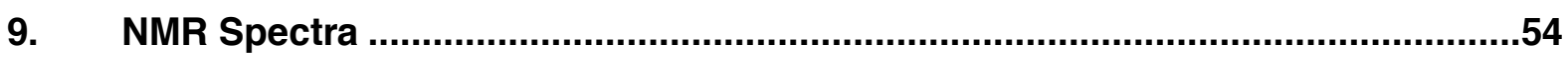

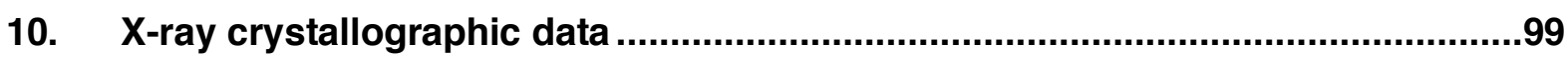

11. Supporting Information Bibliography.............................................................104 


\section{Abbreviations}

(HR)MS high resolution mass spectrometry

Ac

acetyl

ACE-Cl

1-chloroethyl chloroformate

$\mathrm{Ar}$

aromatic

Boc

tert-butyloxycarbonyl

BTC

triphosgene

$\mathrm{Bz}$

benzoyl

$\mathrm{CA}$

cysteamine

CCDC

cambridge crystallographic data centre

Cys

L-Cysteine

DAD

diode array detector

DBU

diazabicycloundecene

DCM

dichloromethane

$\mathrm{DDQ}$

1,2-dichloro-4,5-dicyano-1,4-benzoquinone

DIAD

diisopropyl azodicarboxylate

DIPEA

$N, N$-diisopropyl- $N$-ethyl-amine

DMF

$\mathrm{N}, \mathrm{N}$-dimethylformamide

DMSO

dimethylsulfoxide

DTT

dithiothreitol

EDTA

ethylendiaminetetraacetic acid

El

electron ionisation

em

emission

eq

equivalents

ESI

electron spray ionisation

ESIPT

excited state intramolecular proton transfer

Et

ethyl

ex

excitation

$\mathrm{F}$

(raw) fluorescence intensity

FCC

flash column chromatography

GR

glutathione reductase

Grx

glutaredoxin

$\mathrm{GSH}$

glutathione

GSSG

glutathione disulfide

HPLC

high pressure liquid chromatography 


\begin{tabular}{|c|c|}
\hline IG/IG-OH & $\begin{array}{l}\text { 2',7'-difluoro-3'-hydroxy-6'-isopropoxy-3H-spiro[isobenzofuran-1,9'- } \\
\text { xanthen]-3-one }\end{array}$ \\
\hline$i \operatorname{Pr}$ & iso-propyl \\
\hline$J$ & coupling constant \\
\hline $\mathrm{Me}$ & methyl \\
\hline MEDA & mercaptoethyl-dimethylamine \\
\hline MF/MF-OH & 3'-hydroxy-6'-methoxy-3H-spiro[isobenzofuran-1,9'-xanthen]-3-one \\
\hline $\mathrm{MR} / \mathrm{MR}-\mathrm{NH}_{2}$ & 3'-amino-6'-methoxy-3H-spiro[isobenzofuran-1,9'-xanthen]-3-one \\
\hline Ms & methanesulfonyl \\
\hline MW & microwave \\
\hline NAC & L-Acetyl L-Cysteine \\
\hline NADPH & nicotinamide adenine dinucleotide phosphate \\
\hline NMR & nuclear magnetic resonance \\
\hline $\mathrm{Ph}$ & phenyl \\
\hline PMB & 4-methoxybenzyl \\
\hline $\mathrm{PQ} / \mathrm{PQ}-\mathrm{OH}$ & 6-chloro-2-(5-chloro-2-hydroxyphenyl)quinazolin-4(3H)-one \\
\hline r.t. & room temperature \\
\hline $\mathrm{R}_{f}$ & retention factor \\
\hline$r f x$ & reflux \\
\hline Ser & L-Serine \\
\hline SS-Bz & disulfide containing benzamide series \\
\hline TCEP & tris(2-carboxyethyl)phosphine \\
\hline TE & tris(hydroxymethyl)aminomethan hydrochlorid (Tris-HCI)/EDTA \\
\hline TES & 2-[[1,3-dihydroxy-2-(hydroxymethyl)propan-2-yl]amino]ethanesulfonic acid \\
\hline TFA & trifluoroacetic acid \\
\hline THF & tetrahydrofuran \\
\hline TLC & thin layer chromatography \\
\hline TRP14 & thioredoxin-related protein of $14 \mathrm{kDa}$ \\
\hline $\operatorname{Trx}$ & thioredoxin \\
\hline TrxR & thioredoxin reductase \\
\hline Ts & 4-toluolsulfonyl \\
\hline U498C & selenocysteine (Sec498) to cysteine mutation \\
\hline xantphos & (9,9-dimethyl-9H-xanthene-4,5-diyl)bis(diphenylphosphane) \\
\hline
\end{tabular}




\section{Disulfide-based bioimaging and prodrug approaches}

a imaging agents: linear trigger

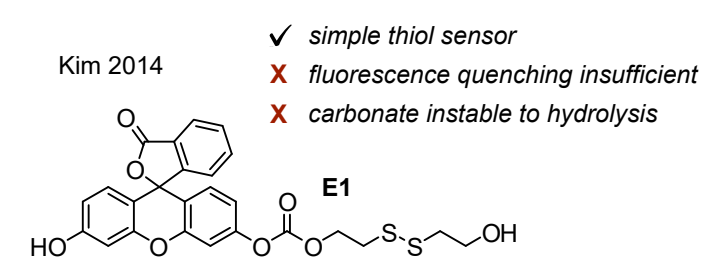

b prodrugs: linear trigger
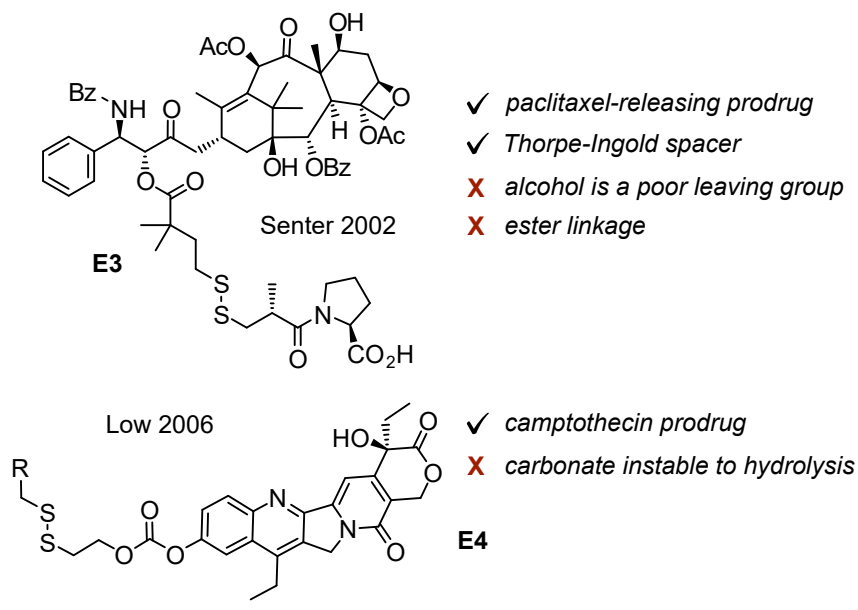

d prodrugs: cyclic trigger

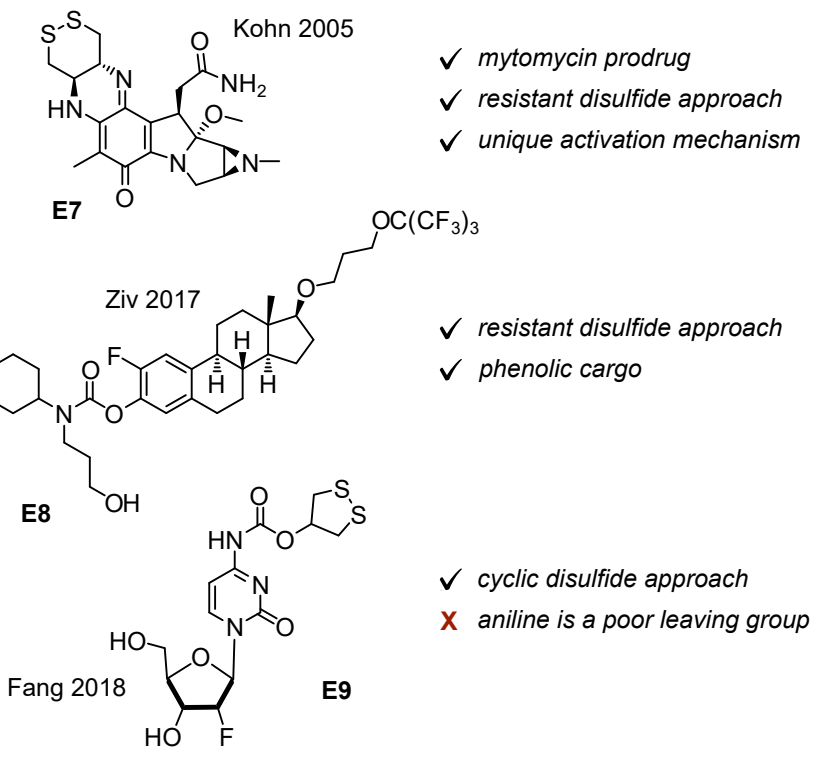

Figure S1 Disulfide-based bioimaging and prodrug approaches: representative recent examples from the literature. (a) linear disulfide based imaging agents for thiol sensing from $\mathrm{Kim}^{1}$ and $\mathrm{Cho}^{2}$. (b) linear disulfide based prodrug approaches from Senter ${ }^{3}$ and Low ${ }^{4}$. (c) cyclic disulfide based bioimaging from Fang ${ }^{5}$ and Tang 6 . (d) cyclic disulfide based prodrug approaches from Kohn ${ }^{7}, \mathrm{Ziv}^{8}$ and Fang ${ }^{9}$. 


\section{Fluorescence properties}

a fluorescence properties

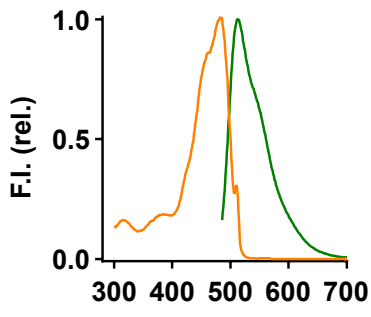

$\lambda(\mathrm{nm})$<smiles>COc1ccc2c(-c3ccccc3C(=O)[O-])c3ccc(=O)cc-3oc2c1</smiles>

MF-O-

483

512

29

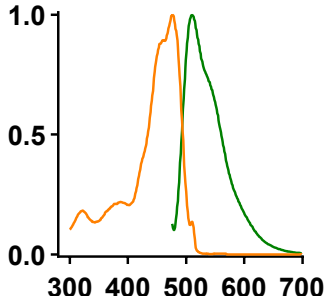

$\lambda(\mathrm{nm})$

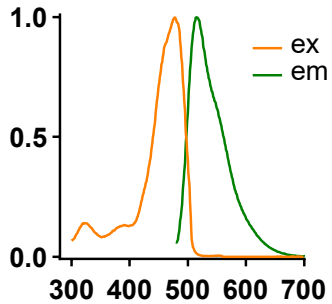

$\lambda(\mathrm{nm})$

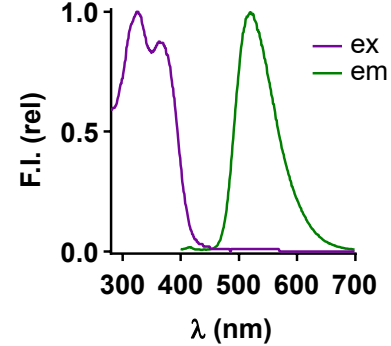<smiles>O=c1[nH]c(-c2cc(Cl)ccc2O)nc2ccc(Cl)cc12</smiles><smiles>COc1ccc2c(-c3ccccc3C(=O)[O-])c3ccc(=N)cc-3oc2c1</smiles>

PQ-OH

$325 / 365$

520

155

b calibration - linearity of fluorescence
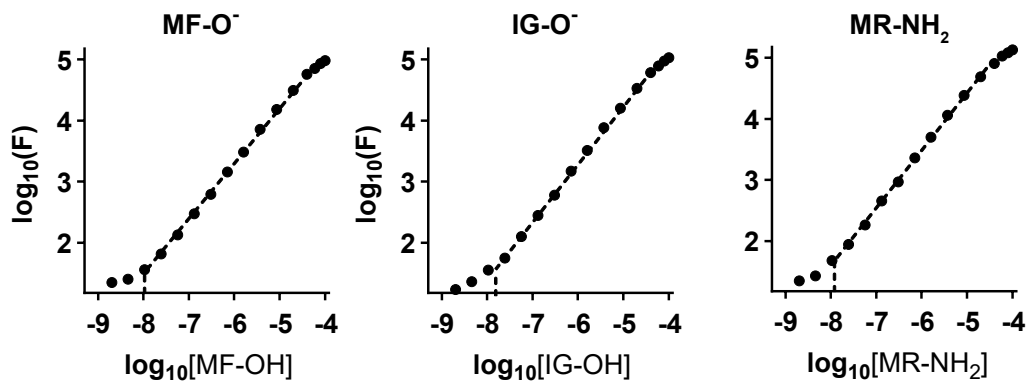

PQ-OH

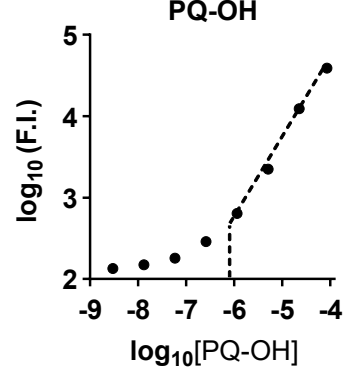

C fluorogenic probe activation - cargo release<smiles>CN(C(=O)Oc1ccc(Cl)cc1-c1nc2ccc(Cl)cc2c(=O)[nH]1)[C@H]1CCSSC1</smiles>
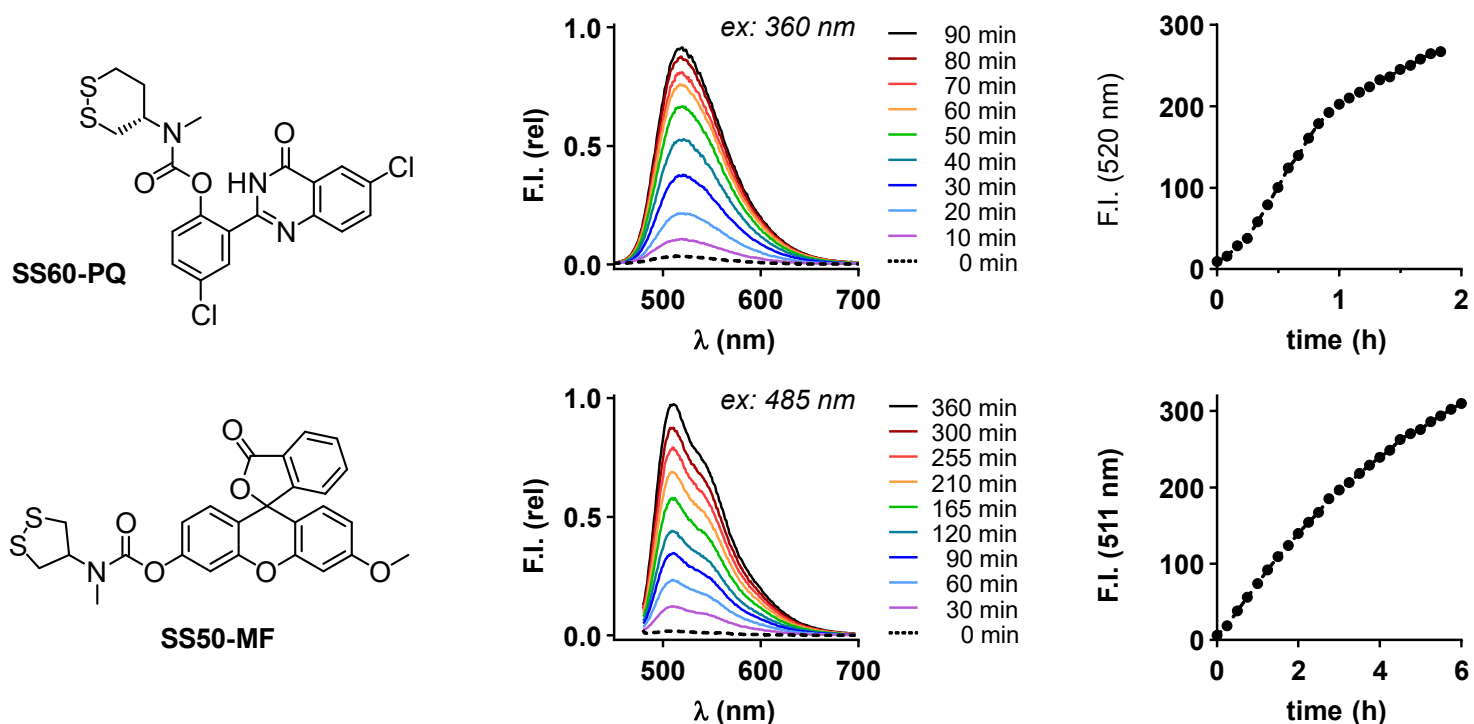

Figure $\mathbf{S 2}$ Photophysical properties of MF-O-, IG-O-, MR- $\mathbf{N H}_{2}$ and $\mathbf{P Q}-\mathbf{O H}$ (a) Excitation and emission spectra (1 $\mu \mathrm{M}$ in TE-buffer, $1 \%$ DMSO).; (b) Calibration: concentration-dependent fluorescence intensity (logarithmic scale $10^{-6}-1 \mathrm{mM}$ in TE-buffer, $1 \%$ DMSO. (c) Off-to-on fluorescence switch-on of SS60-PQ and SS50-MF (2 $\mu \mathrm{M}$ incubated with $1 \mathrm{mM}$ DTT): raw fluorescence intensities: no baseline subtraction (for intact probe). 


\section{Fluorophore selection}

Fluorogenic probes based on the soluble dyes MF-OH, IG-OH or $\mathbf{M R}-\mathbf{N H}_{2}$ should linearly release fluorescent signal upon release, by thiol cyclisation following trigger disulfide reduction. As substitutions of xanthene-based dyes only mildly affect fluorescence properties, MF-OH, IG-OH and $\mathbf{M R - \mathbf { N H } _ { 2 }}$ have similar fluorescence properties, with excitation maximum of $480 \mathrm{~nm}$ and emission band maximum of $510 \mathrm{~nm}$ and small Stokes shift of $30 \mathrm{~nm}$ (Fig. S2a), and they display a consistent linear response range (ca. $15 \mathrm{nM}-100 \mu \mathrm{M}$ ) on a standard platereader with substantially lower detection limit and linear range possible on a fluorimeter. PQ-OH is fluorescent only in the solid state, with excitation maximum $365 \mathrm{~nm}$ and emission band maximum of $520 \mathrm{~nm}$ (Fig. S2b). PQ-based probes therefore generate signal only after local accumulation of released PQ-OH passes its solubility limit, and their fluorescence signal timecourse additionally depends on kinetics of precipitation of PQ-OH. Since fluorescence in the assay is inhomogenous (suspended/sedimented solid) there is potential that the probes' fluorescence signal does not necessarily correspond to released PQ-OH concentration (e.g. up to solubility limit, no signal seen (can be corrected for in analysis, but we found this not necessary and did not do it); past the solubility limit, size-and-morphology-dependent Lambert-Beer shielding by variable optical path length through a solid particle or stack of particles can add nonlinearity - see discussion in ${ }^{10}$; etc). However, we cross-checked a variety of conditions and confirmed precise linear response from ca. $1-100 \mu \mathrm{M}$ (Fig S2b) on a standard fluorescence platereader, matching the cell-free PQ-OH precipitation threshold of just under $1 \mu \mathrm{M}$.

\section{Properties of cargo release of fluorogenic probes}

We next confirmed the true off-to-on switch character of SS60-PQ and SS50-MF as two model representatives and investigated their signal increase kinetics. At low concentrations $(1 \mu \mathrm{M}$ in buffered aq. solution) fluorescence intensity (at $511 \mathrm{~nm}$ and $520 \mathrm{~nm}$ respectively) was close to zero, whereas a constant increase of intensity in the spectra were measured upon incubation with a strong model reductant (1 mM DTT). Signal increase at the emission maximum matched expectations (Fig S2b), as a short delay of steep increase at the very start was observed only for the PQ-OH releasing probe (while the released amount reaches the precipitation threshold), whereas a linear increase with definite slope was detected for the MF-OH releasing probe due to continuous liberation of the dye. Significant differences on the release kinetics were visible as similar fluorescence intensities were accessible after $2 \mathrm{~h}$ for the PQ-OH releasing probe and after $6 \mathrm{~h}$ for the MF-OH releasing probe. 


\section{Relative kinetics of cyclisation}
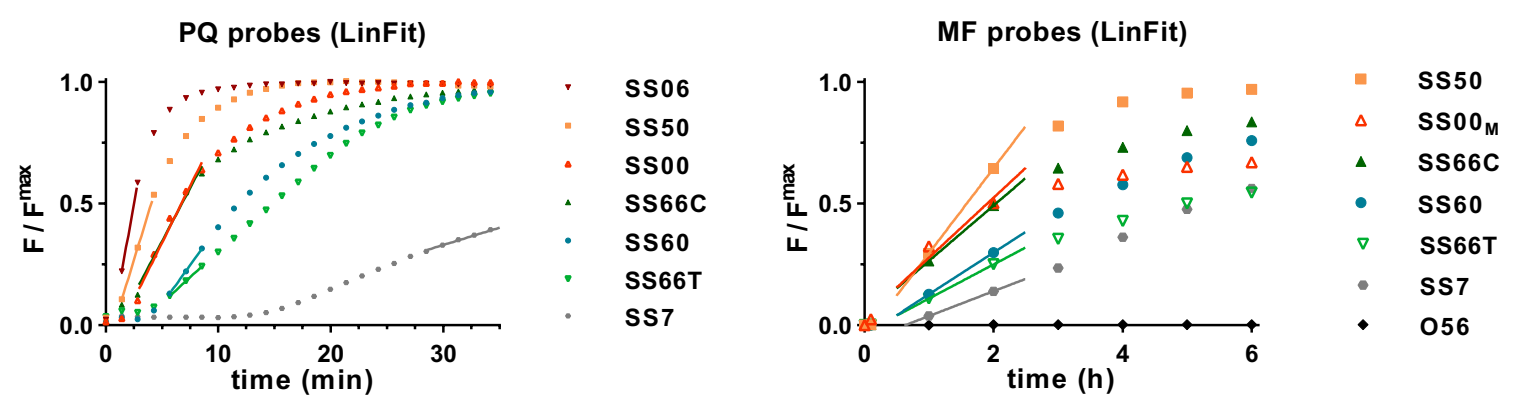

\begin{tabular}{c|cccccccc} 
& & SS00/ SS00M & SS06 & SS60 & SS66C & SS66T & SS50 \\
\hline $\begin{array}{c}\text { initial } \\
\text { slope }\end{array}$ & PQ & 0.093 & 0.255 & 0.064 & 0.088 & 0.043 & 0.150 & 0.014 \\
\hline \multirow{2}{*}{$\begin{array}{c}\text { relative } \\
\text { to SS60 }\end{array}$} & MP & 0.177 & $/$ & 0.157 & 0.257 & 0.131 & 0.338 & 0.073 \\
& MF & 1.44 & 3.96 & 1 & 1.36 & 0.66 & 2.33 & 0.22 \\
& & 1.13 & $/$ & 1 & 1.63 & 0.83 & 2.15 & 0.46
\end{tabular}

Figure S3: Linear fitting to initial release rates for relative rate comparison between triggers (see Fig 6b).

Approximate comparison of the cyclisation rates between different triggers (Fig 6b) was performed by linear fitting to the earliest linear region of initial signal generation timecourses upon treatment with excess of quantitative reductant tris(2-carboxyethyl)phosphine (TCEP) (Fig S3). The logic is that excess of TCEP should rapidly, quantitatively and irreversibly reduce the monomeric disulfide probes; and within a series, all elimination rates from the tetrahedral thiol cyclisation intermediate will be identical; so the relative linear fits should report on the relative rates of thiol cyclisation as long as the initial reduction is indeed rapid.

Relative trigger rates: (a) excellent agreement between the determined from each series of probes was found (Fig 6b, Fig S3) despite the very different actual release rates (that are modified by cargo elimination rate: faster for $\mathbf{P Q}$, slower for $\mathbf{M F}$ ). Other features of the relative rates that are of design interest include: (b) the rate of cyclisation of the SS50-type trigger is almost exactly twice that of SS00-type triggers, matching expectations as it has two rather than one cyclisable groups, but is otherwise identically disposed for cyclisation. The SS7-type trigger also has two cyclisable groups, but it is much slower than either SS50- or SS00-type trigger. We take this to reflect poor kinetics of TCEP reduction of the probe (note very slow reaction with DTT also in the equilibration tests); by analogy to previous work on SS50-type motifs ${ }^{11}$ we assign this to oligomerised probe with poor reductant access. (c) Annelation-preorganised SS06-type trigger cyclisation is 3 times faster than that of linear SS00-type triggers. (d) The post-reduction intermediates (C in Fig 2b) of SS06-, SS66C-, and SS66T-type motifs should be roughly similar in their speed to cyclise; so do the post-reduction intermediates of SS60- and SS00-type. As significant differences are seen between these sets, we conclude that SS66T- and SS66C-type triggers are ca. 3-6-fold slower to be reduced by TCEP than SS06-type; while the SS60-type trigger is only ca. $20 \%$ slower to be reduced by TCEP than linear SS00-type triggers.

Relevance to Mechanism: With TCEP, SS60-PQ has ca. 12 min release halftime and SS60-MF ca. 200 min. The other triggers similarly have a ca. 16-fold faster rate as PQ-conjugates across all triggers. We conclude that the major rate-limiting aspect for signal generation with TCEP is thiol cyclisation and cargo elimination; and that this takes minutes to hours. Therefore we are confident that on-reductant cyclisation of the intermediates $\mathbf{B}$ and $\mathbf{D}$ (if either would directly cyclise to release $\mathrm{PhOH}$ before full reduction to $\mathbf{C}$; Fig 2 ) is not a major contributor to released cargo quantities under expected lifetimes of the transthiolated intermediates (while we cannot estimate those lifetimes from our data, we note the work of Matile ${ }^{12}$ measuring halftimes of just $7 \mathrm{~ms}$ at $25^{\circ} \mathrm{C}$ of a comparable intramolecular thiol/disulfide transthiolation as would be needed to regenerate $A$ by retroaddition of $B / D$ ). 


\section{Evaluation of redox potentials using the SS-Bz series}

\section{General protocols}

HPLC-grade $\mathrm{MeOH}$ and TE buffer (50 mM Tris- $\mathrm{HCl}, 1 \mathrm{mM}$ EDTA, $\mathrm{pH}$ 7.4) were separately degassed by bubbling inert gas for $1 \mathrm{~h}$. Solutions of SS-Bz ${ }^{\text {ox }}(2 \mathrm{mM}$ or $5 \mathrm{mM}$ in degassed $\mathrm{MeOH})$ and DTT red $(2 \mathrm{mM}, 5 \mathrm{mM}$ or $10 \mathrm{mM}$ in degassed TE) were prepared freshly, then in a pre-dried HPLC vial $75 \mu \mathrm{L}$ of the SS-Bz ${ }^{\text {ox }}$ solution was mixed with $75 \mu \mathrm{L}$ of DTTred solution. Several HPLC based measurements were conducted with $10 \mu \mathrm{L}$ injecting volume at different timepoints. Relative ratio of SS-Bz ${ }^{\mathrm{ox}}$ and SS-Bz ${ }^{\text {red }}$, as well as DTT ${ }^{\text {red }}$ and DTT ${ }^{\text {ox }}$ calculated from the HPLC chromatogram areas.

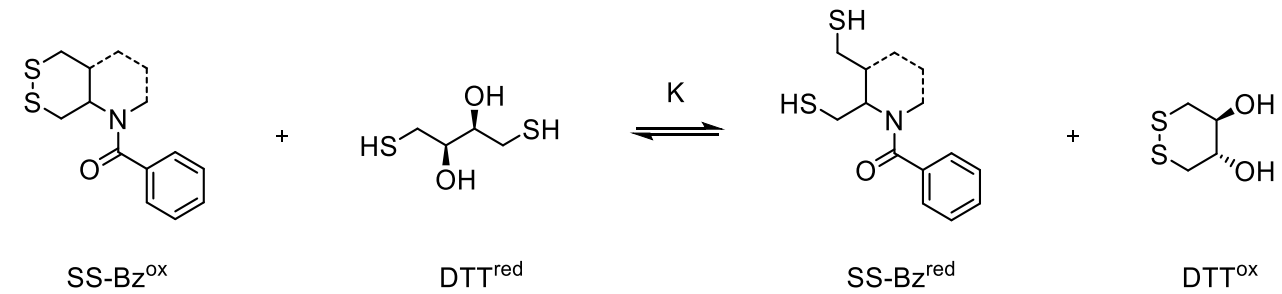

The HPLC-based measurement of relative reduction potentials is based on the fully reversible bimolecular reaction equilibrium between oxidized disulfide-type probes tagged with a UV active tracer (Bz-Series) and the dithiol-type reducing agent DTT. Due to strong polarity differences disulfide and dithiol species can easily be separated by standard HPLC methods and well quantified by integration of the peaks' UV absorption traces at $210 \mathrm{~nm}$ for DTT or $230 \mathrm{~nm}$ for SS-Bz derivatives. Absorption spectra and intensities of oxidized/reduced species of DTT and the SS-Bz derivative were compared at the respective wavelength to ensure the suitability of the measurement for quantification. The method can only be applied if no other reaction products or UV active species are detected.

(eq. 1)

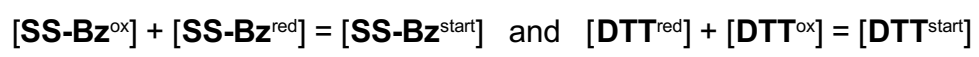

(eq. 2) for equimolar amounts:

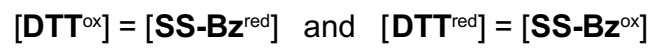

for equimolar amounts,

with (eq. 2):

with (eq. 1):

thus (eq. 3):

for non-equimolar amounts, with (eq. 1):

thus (eq. 4):

$$
\mathrm{E}_{[\mathrm{SS}-\mathrm{Bz}]}^{0}=\mathrm{E}_{[\mathrm{DTT}]}^{0}-\frac{\mathrm{R} \cdot \mathrm{T}}{\mathrm{n} \cdot \mathrm{F}} \ln \frac{\left[\mathrm{DTT}^{\mathrm{red}}\right] \cdot\left[\mathrm{SS}-\mathrm{Bz}^{\mathrm{ox}}\right]}{\left[\mathrm{DTT}^{\mathrm{ox}}\right] \cdot\left[\mathrm{SS}-\mathrm{Bz}^{\mathrm{red}}\right]}
$$

$E_{[S S-B z]}^{0}=E_{[D T T]}^{0}-\frac{R \cdot T}{n \cdot F} \ln \frac{\left[S S-B z^{\text {red }}\right]^{2}}{\left[S S-B z^{o x}\right]^{2}}$

$$
{E^{[S S-B z]}}^{0}=E_{[D T T]}^{0}-\frac{R \cdot T}{n \cdot F} \ln \frac{\left(\% S S-B z^{\text {ox }} \cdot\left[S S-B z^{\text {start }}\right]\right)^{2}}{\left(\% S S-B z^{\text {red }} \cdot\left[S S-B z^{\text {start }}\right]\right)^{2}}
$$

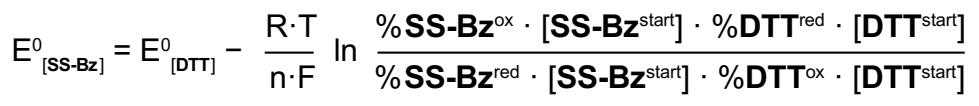

$\mathrm{E}_{\text {[SS-Bz] }}^{0}=\mathrm{E}_{[\text {[DT] }}^{0}-\frac{\mathrm{R} \cdot \mathrm{T}}{\mathrm{n} \cdot \mathrm{F}} \ln \frac{\% \mathrm{SS}-\mathrm{Bz}^{\mathrm{ox}} \cdot \% \mathrm{DTT}^{\mathrm{red}}}{\% \mathrm{SS}-\mathrm{Bz}^{\mathrm{red}} \cdot \% \mathrm{DTT}^{\mathrm{ox}}}$ 


\section{Evaluation of reduction mediated release}

\section{General protocol}

Cell-free in vitro assays were performed to evaluate the fluorogenic probes' performance based on reduction-mediated (chemical reductants) release of their fluorescent cargo. The molecular process was monitored by fluorescence intensity increase based on the off-to-on mechanism both for $\mathbf{P Q}-\mathbf{O H}_{(\mathrm{s})}$ (precipitation-based induction of ESIPT fluorescence monitored with ex/em 355bp10/520lp) and for MF-OH (aq), IG-OH (aq) and MR-NH $\mathbf{N}_{2}$ (aq) (conjugation-based induction of long-wavelength absorption and fluorescence monitored with ex/em 485bp10/520lp).

In a black 96 -well plate with black bottom, $80 \mu \mathrm{L}$ of a diluted solution $(12.5 \mu \mathrm{M}$ in aq. TE, $\mathrm{pH} 7.4,1.25 \%$ DMSO) of the PQ-, MF-, IG- or MR-based fluorogenic probe (to reach a final concentration of $10 \mu \mathrm{M}$ ) was mixed with $20 \mu \mathrm{L}$ of a solution of selected chemical reductants $(50 \mu \mathrm{M}$ to $50 \mathrm{mM}$ in aq. TE, pH 7.4 to reach $10 \mu \mathrm{M}$ to $10 \mathrm{mM}$ ). The reaction mixtures were incubated at $37^{\circ} \mathrm{C}$ and $100 \%$ humidity for $6 \mathrm{~h}$.

Timecourse measurements were conducted to determine kinetics of the reduction-mediated release. Data was interpreted by calculating the absolute, time-dependent fluorescence intensity normalised to the plateau signal observed in a reference maximum-fluorescence experiment $F^{\max }(\mathbf{t})$ that used incubation with an excess of tris(carboxyethyl)phosphine (TCEP) for PQ-probes. For MF-, IG- and MR-based probes the absolute, time-dependent fluorescence intensity was normalized according to a separate calibration experiment using the free, soluble fluorophores MF-OH, IG-OH and $\mathbf{M R}-\mathbf{N H}_{\mathbf{2}}$.

\section{Notes}

Since small differences in assay setup affect the kinetics in the early phase, for each graph we report TCEP control reduction assays that were run on the same plate under the same conditions, rather than averaged behaviour from other runs. The TCEP run kinetics thus differ slightly between different reduction series tests, but can be used to more accurately compare to outcomes in the series reported. Further, we noted that upon standing, both SS-50-type and SS-7-tpye probe stocks had reduced capacity to generate fluorescence in reductant assays. This may be attributed to the formation of ringopened and relatively hydrophobic polymer species with reduced accessibility to aqueous reductants, upon standing in concentrated solutions, as Houk and Whitesides observed for similar species. ${ }^{13}$ Consequently, their probe stocks were prepared freshly from the solids for all later assays. The linear or 6-membered disulfide probes did not display this instability.

\section{Chemoreductants}

\begin{tabular}{ccc} 
reductant & c $(\mathrm{mM})$ & eq. (reductant/probe) \\
\hline TCEP & $0.1-3$ & $10-300$ \\
DTT & $0.01-10$ & $1-1000$ \\
DTT & 1 & 100 \\
GSH & $0.01-10$ & $1-1000$ \\
GSSG, Ser & 1 & 100 \\
Cys, NAC & 1 & 100 \\
MEDA, CA & 1 & 100
\end{tabular}

TCEP: tris(carboxyethyl)phosphine, DTT: dithiothreitol (reduced), DTTox: dithiothreitol (oxidized), GSH: glutathione (reduced), GSSG: glutathione (oxidized), Cys: L-Cysteine, Ser: L-Serine, MEDA: mercaptoethyl-dimethylamine, CA: cysteamine and NAC: $N$-acetyl L-Cysteine. 
a chemoselectivity
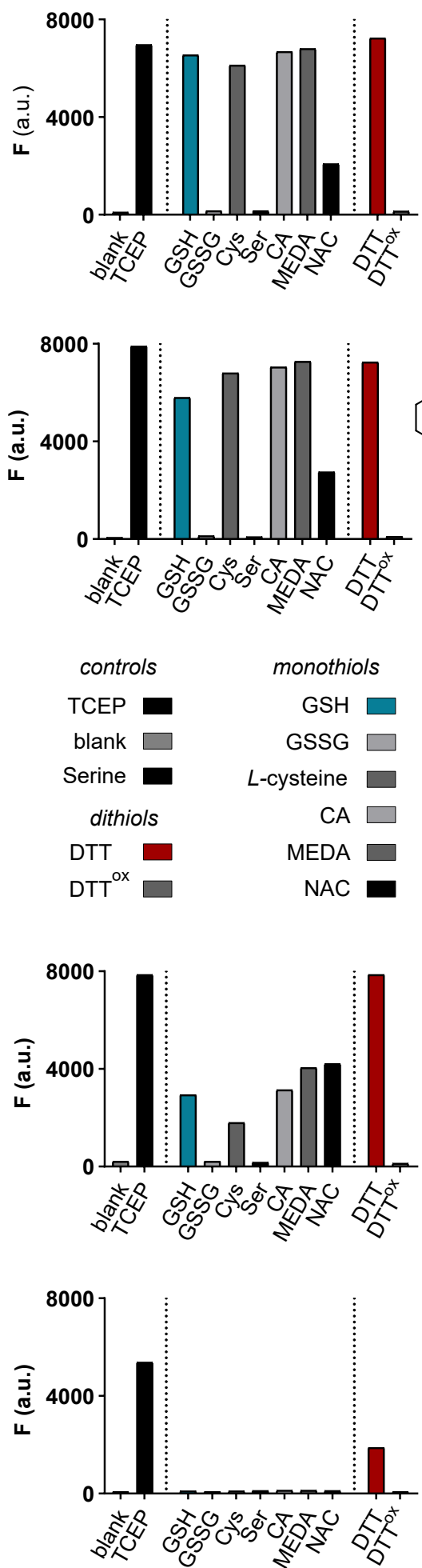

b

monothiol stability

GSH

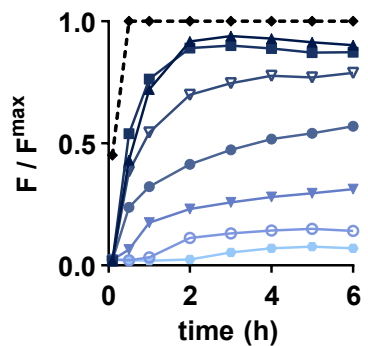

GSH

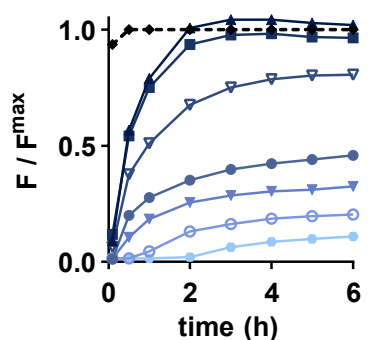

(ii) SS06-PQ

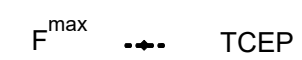

$$
\begin{array}{r}
\frac{e q(G S H)}{1000} \rightarrow \frac{[G S H]}{10 \mathrm{mM}} \\
300 \multimap 3 \mathrm{mM} \\
100 \multimap 1 \mathrm{mM} \\
30 \multimap 0.3 \mathrm{mM} \\
10 \multimap 0.1 \mathrm{mM} \\
3 \multimap 30 \mathrm{mM} \\
1 \multimap 10 \mathrm{mM}
\end{array}
$$

GSH<smiles>CCC(=O)N(C)C1CSSC1</smiles>

(iii) SS50-PQ

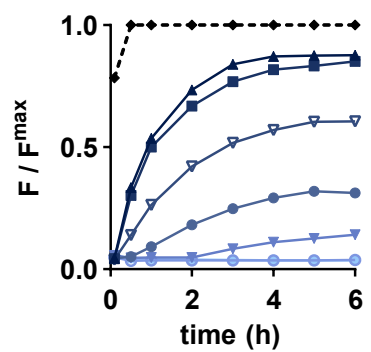

GSH

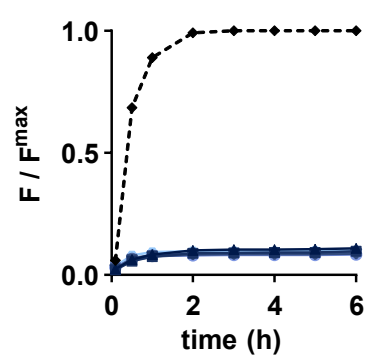

C dithiol activation

DTT

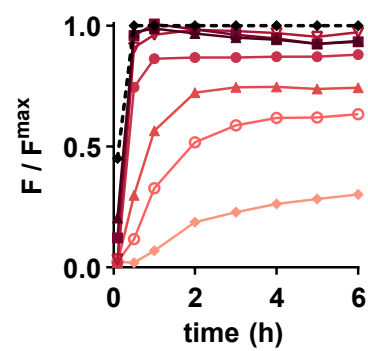

DTT

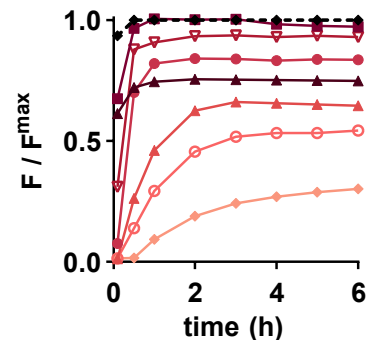

$F^{\max } \ldots \quad$ TCEP

$$
\begin{aligned}
& \frac{e q(D T T)}{1000} \leftarrow \frac{[D T T]}{10 \mathrm{mM}} \\
& 300 \rightarrow 3 \mathrm{mM} \\
& 100 \rightarrow 1 \mathrm{mM} \\
& 30 \rightarrow 0.3 \mathrm{mM} \\
& 10 \simeq \quad 0.1 \mathrm{mM} \\
& 3 \rightarrow 30 \mathrm{mM} \\
& 1 \div \quad 10 \mathrm{mM}
\end{aligned}
$$
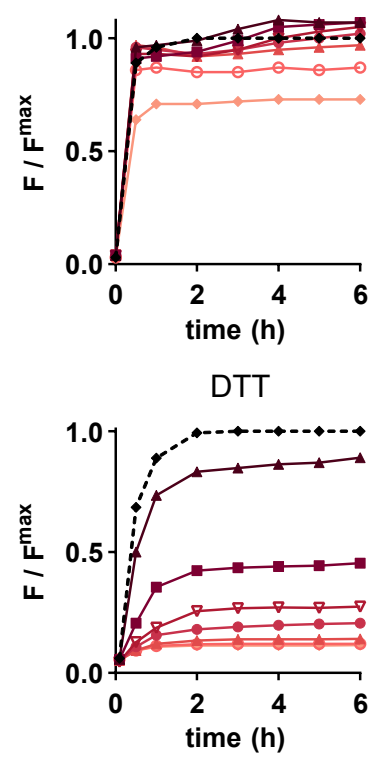

Fig. S4 Evaluation of reduction mediated cargo release of (i) SS00-PQ, (ii) SS06-PQ, (iii) SS50-PQ and (iv) SS7-PQ (10 $\mu \mathrm{M}$ in TE-buffer). (a) Fluorescence signal after $2 \mathrm{~h}$ upon incubation with the following reductants: TCEP $(100 \mu \mathrm{M})$, DTT (1 mM), DTT ${ }^{\text {ox }}(1 \mathrm{mM})$, GSH (1 mM), GSSG (1 mM), Cys (1 mM), Ser (1 mM), MEDA (1 mM), CA (1 mM) and NAC (1 mM) (b) Relative fluorescence signal 0-6 h upon incubation with (c) GSH and (d) DTT with increasing concentrations $(10 \mu \mathrm{M}, 30 \mu \mathrm{M}, 100 \mu \mathrm{M}, 300 \mu \mathrm{M}, 1 \mathrm{mM}, 3 \mathrm{mM}$ and $10 \mathrm{mM})$ with respect to TCEP $(100 \mu \mathrm{M})$. 
a chemoselectivity
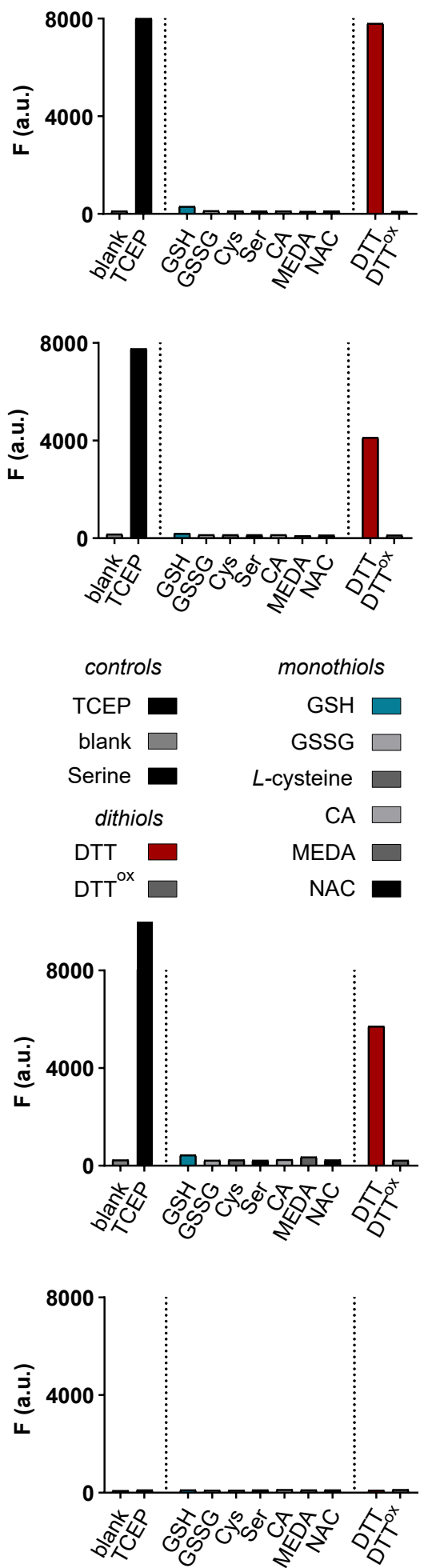

b monothiol stability

GSH<smiles>CC(C)C(=O)N(C)C1CCSSC1</smiles>

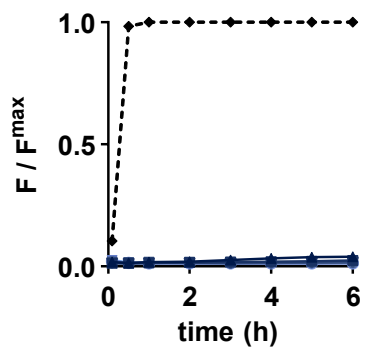

GSH<smiles>CCC(=O)N1CCCC2CSSCC21</smiles>

(vi) SS66C-PQ

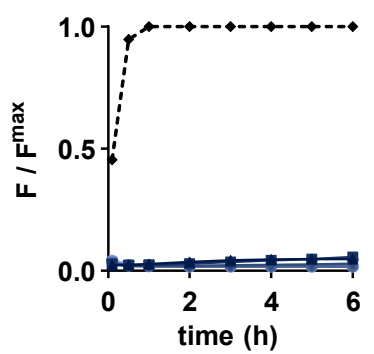

$F^{\max } \ldots \quad$ TCEP<smiles>COc1ccc(Cl)cc1-c1nc2ccc(Cl)cc2c(=O)[nH]1</smiles>

$\frac{e q(G S H)}{1000} \simeq \frac{[G S H]}{10 \mathrm{mM}}$

$300=3 \mathrm{mM}$

$100 \rightarrow 1 \mathrm{mM}$

$30 \rightarrow 0.3 \mathrm{mM}$

$10 \div 0.1 \mathrm{mM}$

$3=30 \mathrm{mM}$

$1=10 \mathrm{mM}$

GSH<smiles>CCC(=O)N1CCC[C@H]2CSSC[C@H]21</smiles>

(vii) SS66T-PQ

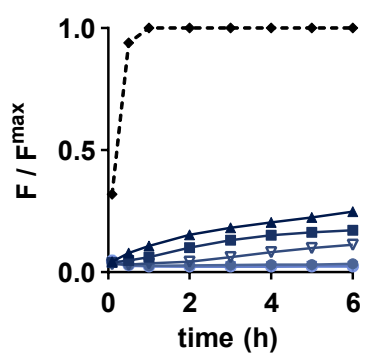

GSH

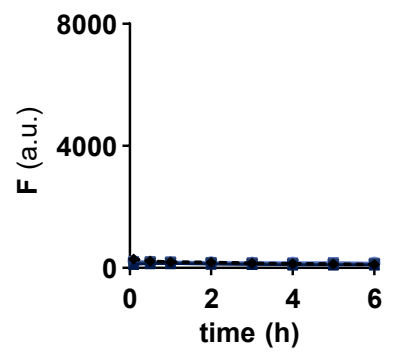

C dithiol activation

DTT

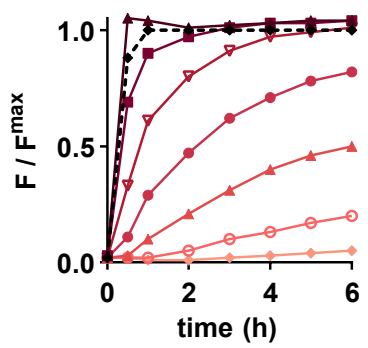

DTT

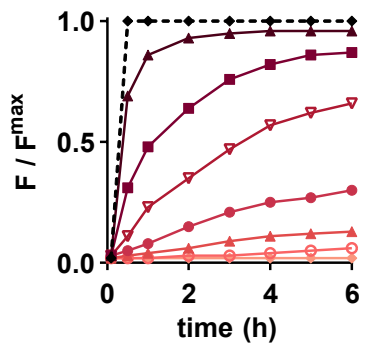

$\mathrm{F}^{\max } \ldots \quad$ TCEP

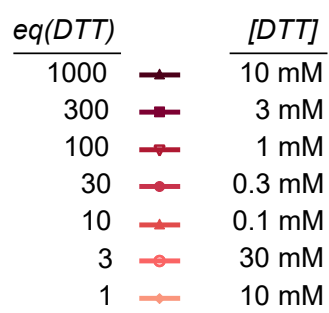

DTT

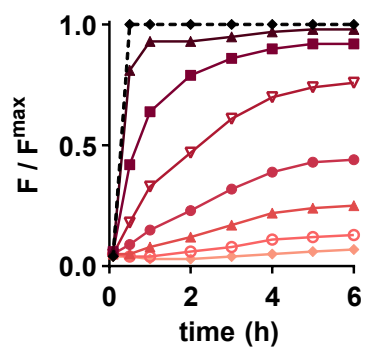

DTT

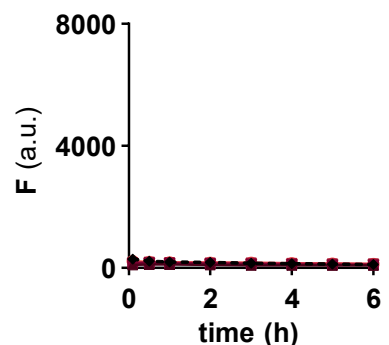

Fig. S5 Evaluation of reduction mediated cargo release of (v) SS60-PQ, (vi) SS66C-PQ, (vii) SS66T-PQ and (viii) O56-PQ (10 $\mu \mathrm{M}$ in TE-buffer). (a) Fluorescence signal after $2 \mathrm{~h}$ upon incubation with the following reductants: TCEP $(100 \mu \mathrm{M})$, DTT (1 mM), DTT ${ }^{\text {ox }}(1 \mathrm{mM}), \mathrm{GSH}(1 \mathrm{mM}), \mathrm{GSSG}(1 \mathrm{mM})$, Cys (1 mM), Ser (1 mM), MEDA (1 mM), $\mathrm{CA}(1 \mathrm{mM})$ and NAC (1 mM) (b) Relative fluorescence signal 0-6 h upon incubation with (c) GSH and (d) DTT with increasing concentrations $(10 \mu \mathrm{M}, 30 \mu \mathrm{M}, 100 \mu \mathrm{M}, 300 \mu \mathrm{M}, 1 \mathrm{mM}, 3 \mathrm{mM}$ and $10 \mathrm{mM})$ with respect to TCEP $(100 \mu \mathrm{M})$. 


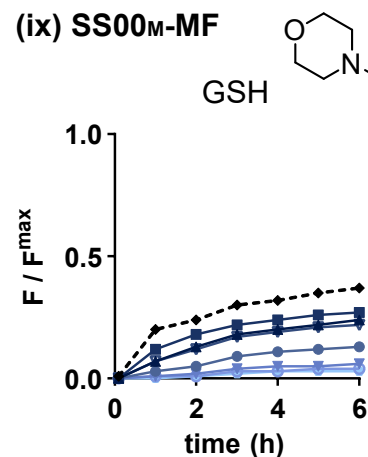

(x) SS50-MF

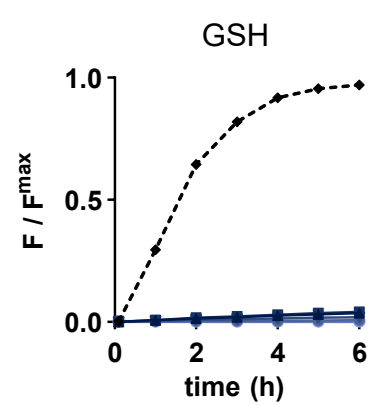

(xi) SS7-MF

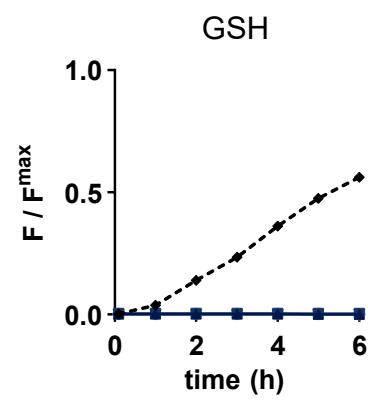

(xii) 056-MF

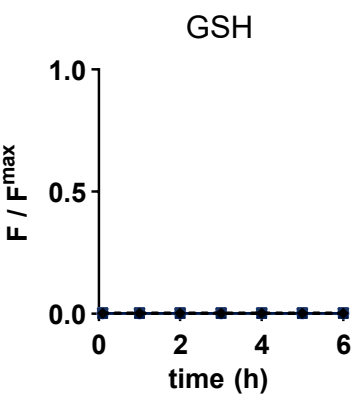

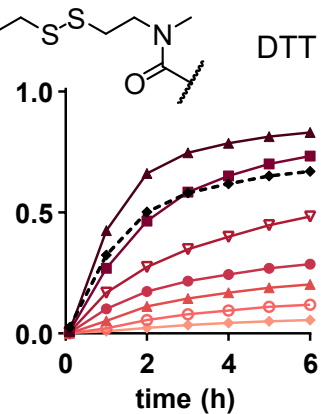<smiles>CNC1CSSC1</smiles>

O DTT

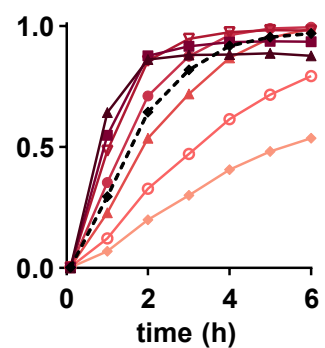

$\left\langle{ }_{N}^{S-S}\right.$
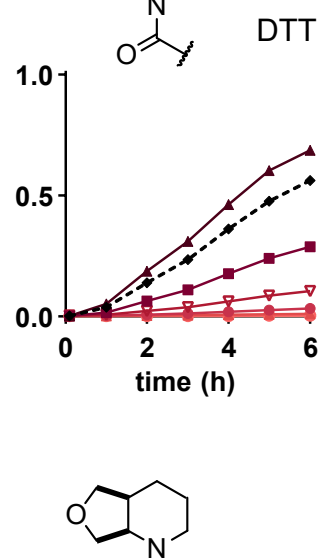

OY DTT

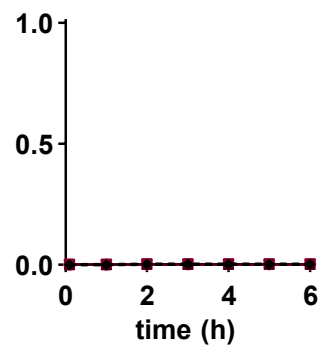

(xiii) SS60-MF
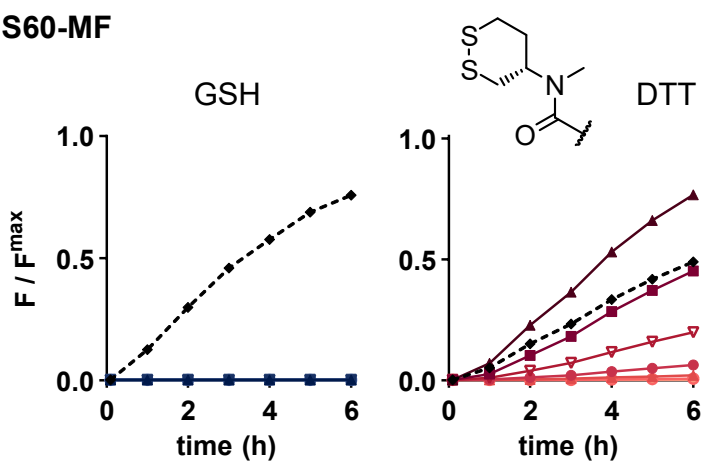

(xiv) SS66C-MF

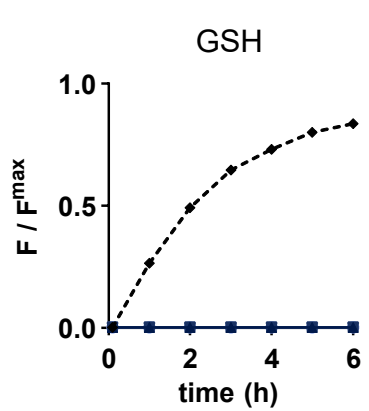

(1)

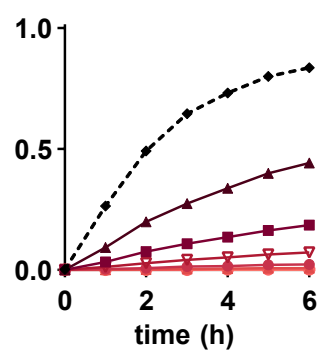

(xv) SS66T-MF

GSH
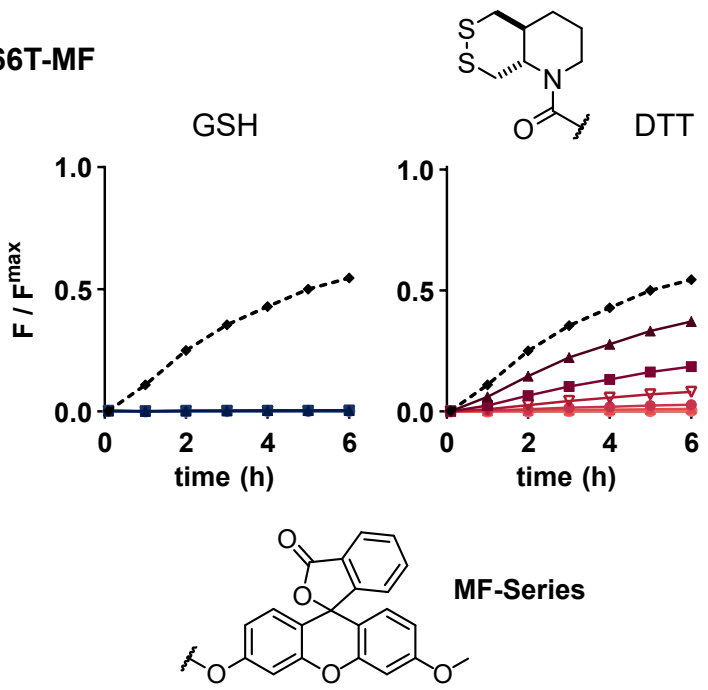

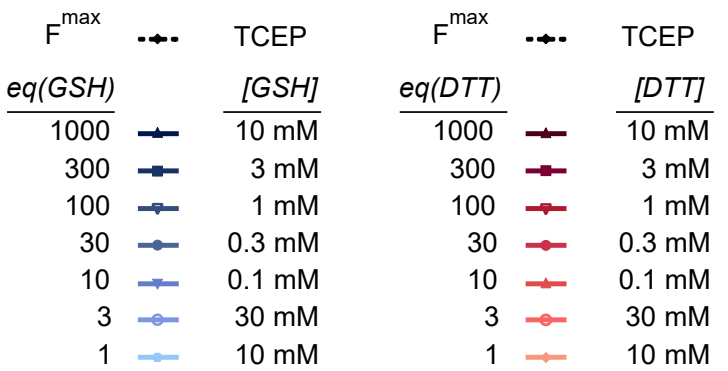

Fig. S6 Evaluation of reduction mediated cargo release of (ix) SS00M-MF, (x) SS50-MF, (xi) SS7-MF, (xii) O56-MF, (xiii) SS60-MF, (xiv) SS66C-MF and (xv) SS66T-MF (10 $\mu$ M in TE-buffer). Relative fluorescence signal (timecourse: 0-6 h) upon incubation with GSH and DTT with increasing concentrations $(10 \mu \mathrm{M}, 30 \mu \mathrm{M}$, $100 \mu \mathrm{M}, 300 \mu \mathrm{M}, 1 \mathrm{mM}, 3 \mathrm{mM}$ and $10 \mathrm{mM})$ with respect to TCEP $(100 \mu \mathrm{M})$ and normalized according to the calibration shown in Fig $\mathbf{S 2}$. 
(xvi) SS00-MF 2

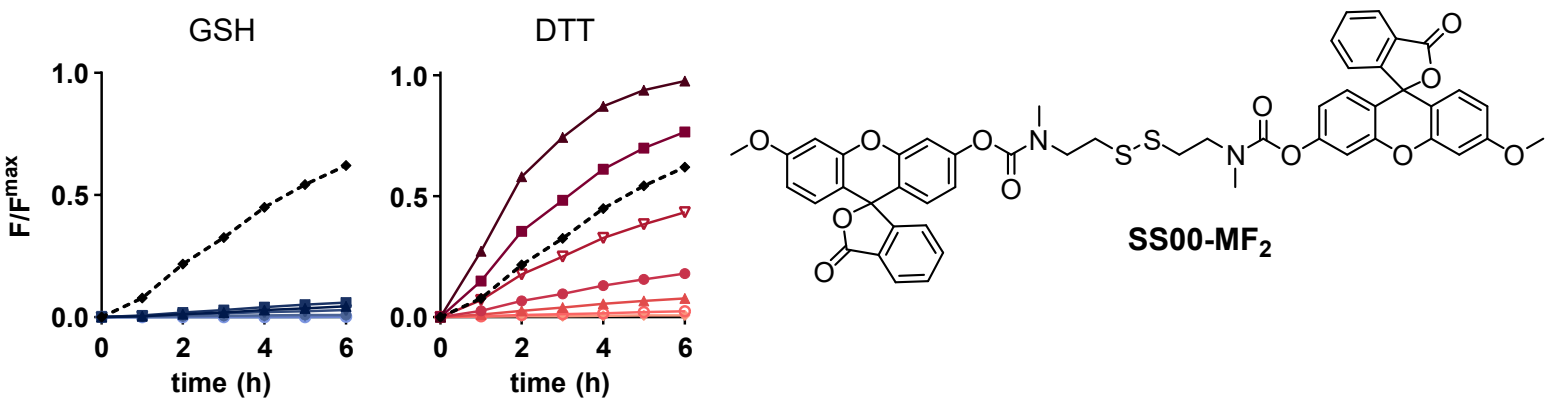

(xvii) SS60-IG

GSH

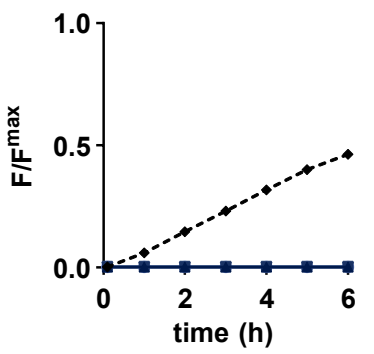

(xix) 056-IG

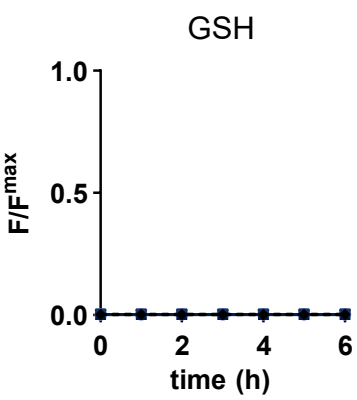

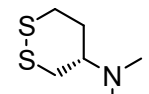
O DTT
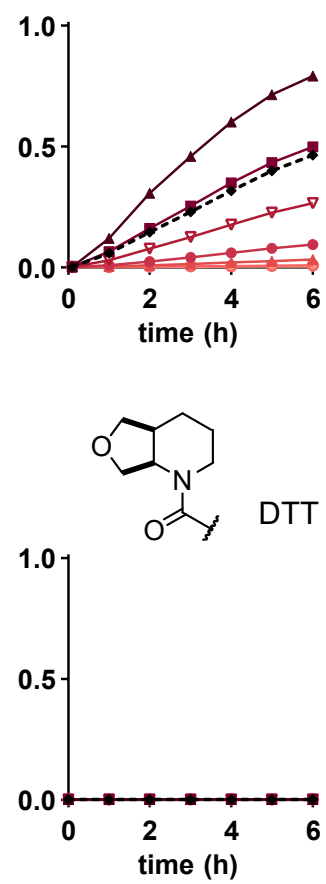

(xviii) SS66C-IG

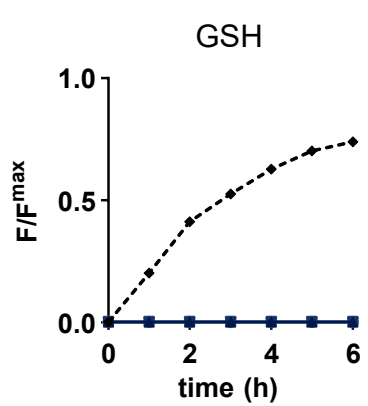

ș O DTT

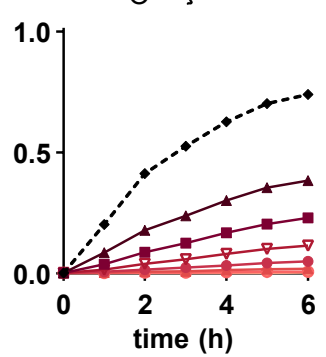

(xx) SS60-MR

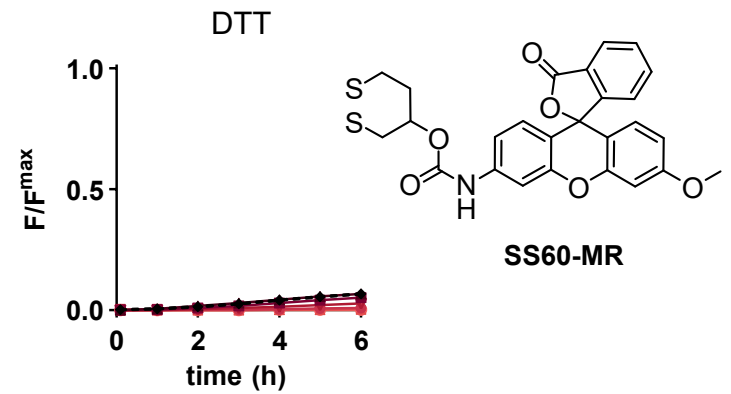

$$
\begin{aligned}
& \mathrm{F}^{\max } \text {... TCEP } \\
& \frac{e q(D T T)}{1000} \simeq \frac{[D T T]}{10 \mathrm{mM}} \\
& 300=3 \mathrm{mM} \\
& 100 \rightarrow 1 \mathrm{mM} \\
& 30 \rightarrow 0.3 \mathrm{mM} \\
& 10 \simeq 0.1 \mathrm{mM} \\
& 3=30 \mathrm{mM} \\
& 1 \div \quad 10 \mathrm{mM}
\end{aligned}
$$

Fig. S7 Evaluation of reduction mediated cargo release of (xvi) SS00-MF 2 , (xvii) SS60-IG, (xviii) SS66C-IG, (xix) 056-IG and (xx) SS60-MR (10 $\mu \mathrm{M}$ in TE-buffer). Relative fluorescence signal (timecourse: $0-6 \mathrm{~h})$ upon incubation with GSH and DTT with increasing concentrations $(10 \mu \mathrm{M}, 30 \mu \mathrm{M}, 100 \mu \mathrm{M}, 300 \mu \mathrm{M}, 1 \mathrm{mM}, 3 \mathrm{mM}$ and $10 \mathrm{mM})$ with respect to TCEP $(100 \mu \mathrm{M})$ and normalized according to the calibration shown in Fig S2. 


\section{Enzyme specifity/activity study}

\section{General protocol}

Cell-free in vitro enzyme specificity assays (bioreductants) were performed to evaluate the fluorogenic probes based on the reduction-mediated release of their fluorescent cargo after incubation with bioreductant cascade model systems. The molecular process was monitored by fluorescence intensity increase based on the off-to-on mechanism for $\mathbf{P Q} \mathbf{O H}_{(\mathrm{s})}$ (precipitation-based induction of ESIPT fluorescence monitored with ex/em 355bp10/520lp) or MF-OH (ex/em 485bp10/520lp).

In a black $96-$ Well Plate with black bottom, $50 \mu \mathrm{L}$ of a diluted solution $(20 \mu \mathrm{M}$ or $2 \mu \mathrm{M}$ in aq. TE, pH 7.4, $2 \%$ DMSO to reach final concentrations of $1 \mu \mathrm{M}$ and $10 \mu \mathrm{M}$ respectively) of the fluorogenic probe was mixed with $40 \mu \mathrm{L}$ of the selected oxidoreductase enzyme (TrxR1, TrxR2, GR to reach final concentrations of $20 \mathrm{nM}$ ) and/or its respective native substrate (Trx1, Trx2, TRP14, Grx1, Grx2 to reach final concentrations of $10 \mathrm{nM}$ to $10 \mu \mathrm{M}$ respectively). The biochemical reaction was started by addition of $10 \mu \mathrm{L}$ of a solution of $\beta$-NADPH ( $1 \mathrm{mM}$ in aq. TE, $\mathrm{pH} 7.4$ to reach final concentrations of $100 \mu \mathrm{M}$ ). The reaction mixtures were incubated at $37^{\circ} \mathrm{C}$ and $100 \%$ humidity for $6-12 \mathrm{~h}$.

Timecourse measurements were conducted to determine kinetics of the reduction-mediated release. Data was interpreted by calculating the absolute, time-dependent fluorescence intensity $\mathbf{F}(\mathbf{t})(\mathbf{a} . \mathbf{u}$.), that was (optionally for $P Q$-type probes) corrected by the background fluorescence $F^{\mathrm{NADPH}}(\mathbf{t})$ (a.u.) caused by autofluorescence of reduced $\beta$-NADPH. Results were compared to the expected maximumfluorescence during a reference experiment $F^{\max }(\mathbf{t})$ that used incubation with an excess of tris(carboxyethyl)phosphine (200 $\mu \mathrm{M}$ TCEP) for internal control.

\section{Bioreductants}

\begin{tabular}{ccc} 
assay factor & c $(\mu \mathrm{M})$ & eq. (reductant/Probe) \\
\hline NADPH & 100 & 10 \\
GSH & 100 & 10 \\
TrxR1, TrxR2 & 0.02 & 0.002 \\
Trx1, Trx2, TRP14 & $0.01-10$ & $0.001-1$ \\
GR & 0.02 & 0.002 \\
Grx1, Grx2 & $0.01-10$ & $0.001-1$
\end{tabular}

NADPH: $\beta$-Nicotinamide adenine dinucleotide phosphate, GSH: glutathione (reduced), TrxR1: recombinant human cytosolic TrxR, TrxR2: recombinant human mitochondrial isoform of TrxR, Trx1: recombinant human cytosolic thioredoxin (Trx), Trx2: recombinant human mitochondrial isoform of Trx, TRP14: recombinant human cytosolic thioredoxin-related protein of $14 \mathrm{kDa}$ (TRP14), GR: recombinant human glutathione reductase (GR), Grx1: recombinant human cytosolic glutaredoxin (Grx1), Grx2: recombinant human cytosolic isoform of Grx. 

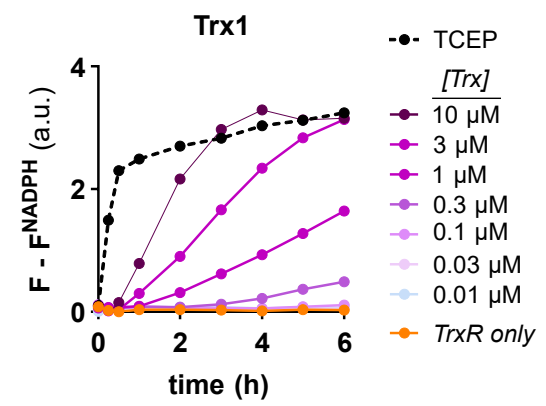

Grx1

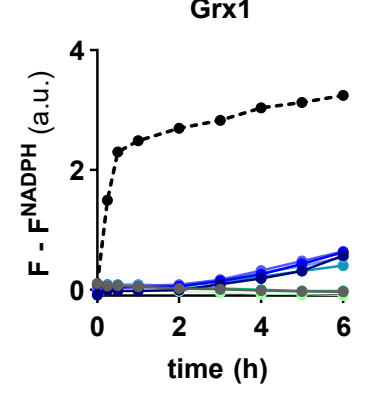

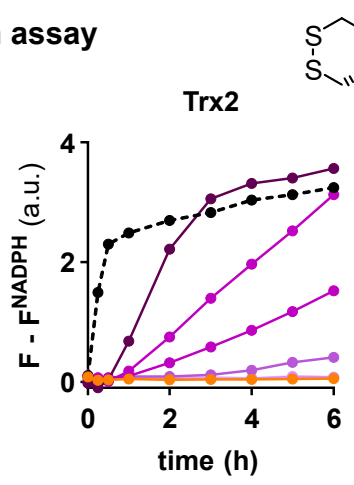

Grx2

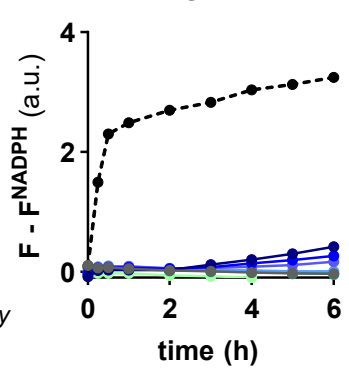

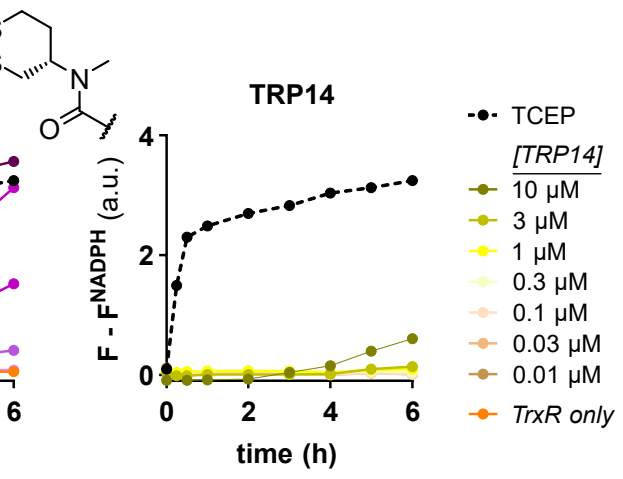

SS60-PQ

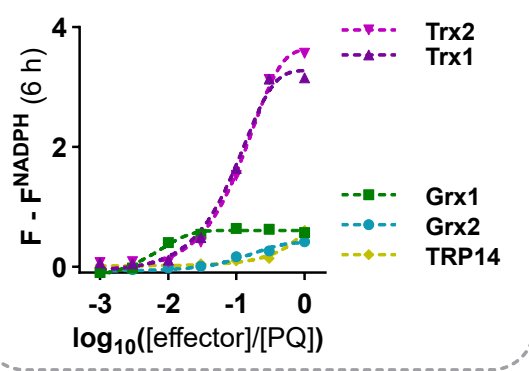

b

SS00-PQ: redox effector titration assay

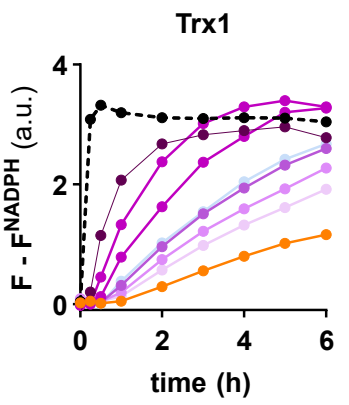

Grx1

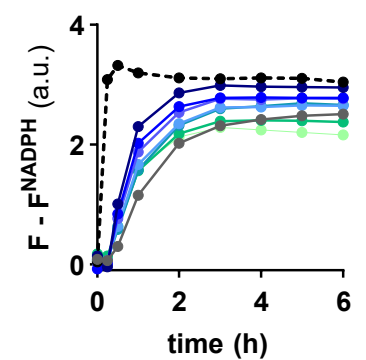

- - TCEP

$\rightarrow \frac{[T r x]}{10 \mu \mathrm{M}}$

- $3 \mu \mathrm{M}$

$\rightarrow 1 \mu \mathrm{M}$

$\rightarrow 0.3 \mu \mathrm{M}$

$-0.1 \mu \mathrm{M}$

$0.03 \mu \mathrm{M}$

$0.01 \mu \mathrm{M}$

$\rightarrow$ TrxR only
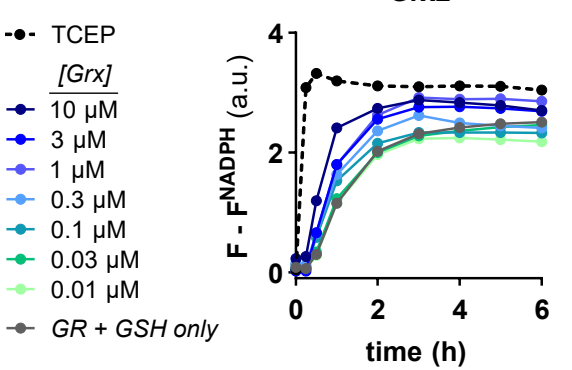

TRP14

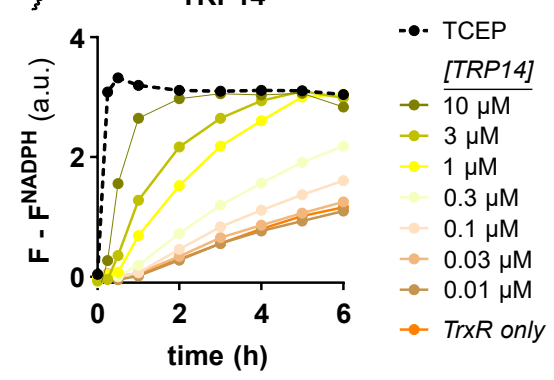

SS00-PQ

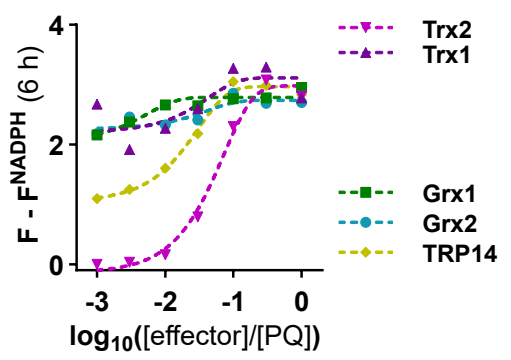

Fig. S8 Redox effector titration assay: (a) Timecourse fluorescence intensity ( $t=0-6 \mathrm{~h}$ ) of SS60-PQ (10 $\mu \mathrm{M})$ upon incubation with Trx1, Trx2, TRP14, Grx1 or Grx2 (0.01-10 $\mu \mathrm{M})$ as compared to a reference experiment: TCEP $(200 \mu \mathrm{M})$. All mixtures additionally containing $\beta$-NADPH $(100 \mu \mathrm{M})$ and the respective upstream system TrxR1, TrxR2 $(20 \mathrm{nM})$ or GR $(20 \mathrm{nM})+\mathrm{GSH}(100 \mu \mathrm{M})$. (b) Same evaluation for SS00-PQ (10 $\mu \mathrm{M})$. 


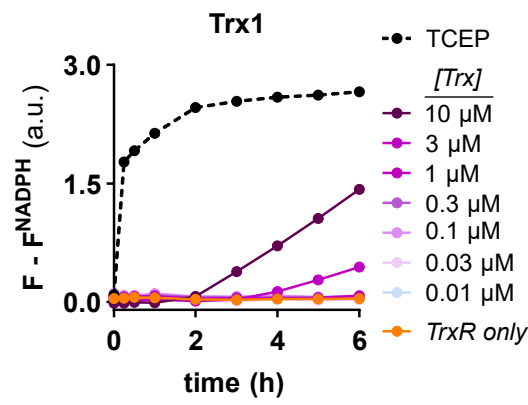

Grx1

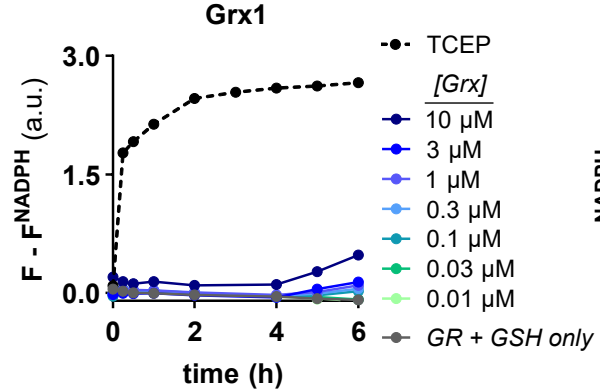

time (h)

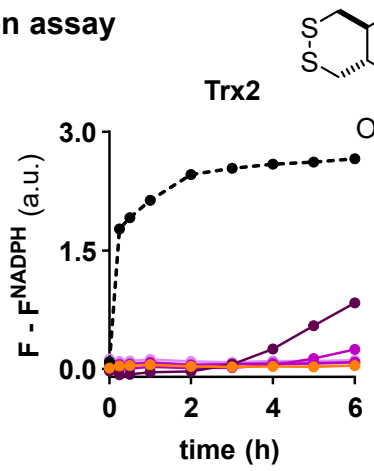

Grx2

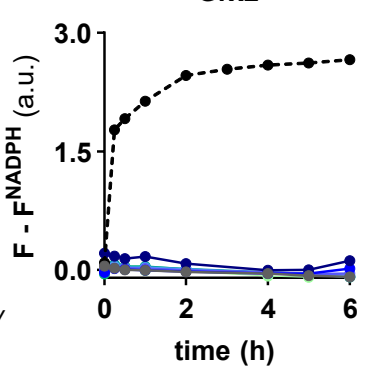

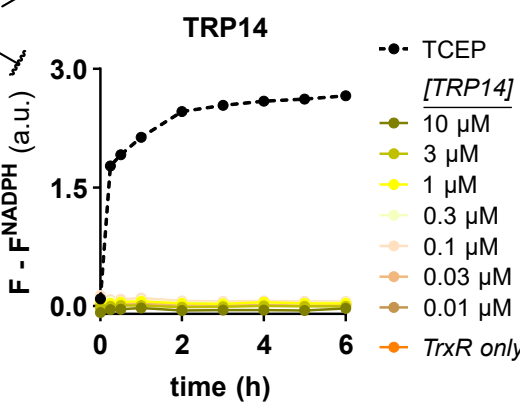

SS66T-PQ

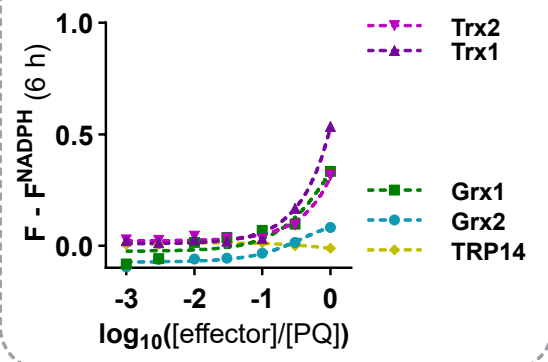

b SS66C-PQ: redox effector titration assay

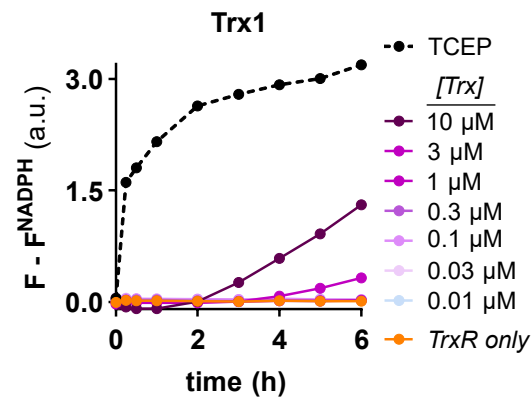

Grx1

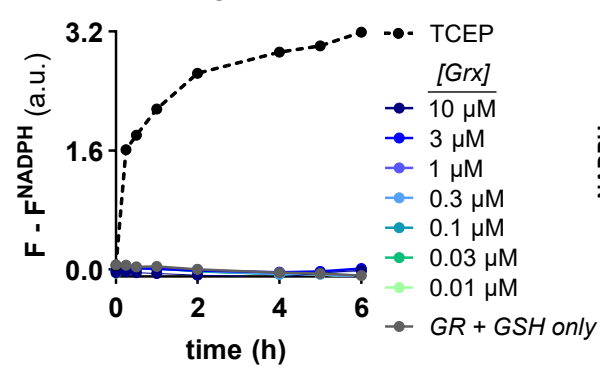

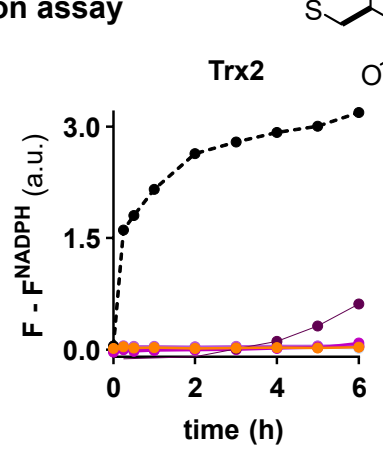

Grx2

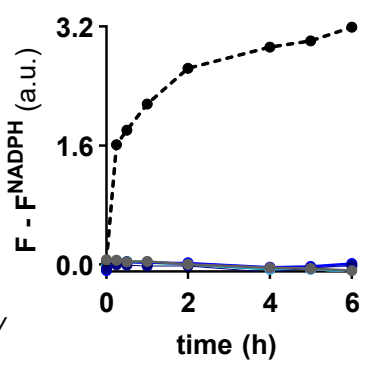

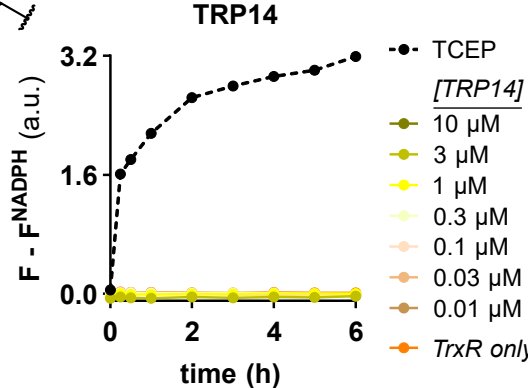

SS66C-PQ

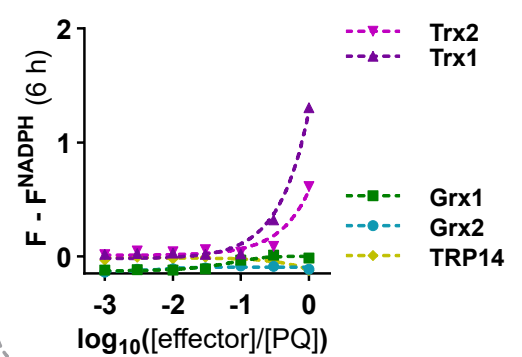

Fig. S9 Redox effector titration assay: (a) Timecourse fluorescence intensity ( $t=0-6 \mathrm{~h}$ ) of SS66T-PQ (10 $\mu \mathrm{M})$ upon incubation with Trx1, Trx2, TRP14, Grx1 or Grx2 (0.01-10 $\mu \mathrm{M})$ as compared to a reference experiment: TCEP $(200 \mu \mathrm{M})$. All mixtures additionally containing $\beta$-NADPH $(100 \mu \mathrm{M})$ and the respective upstream system TrxR1,

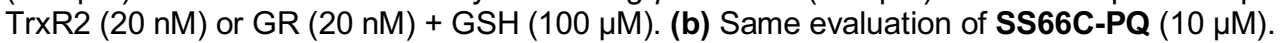




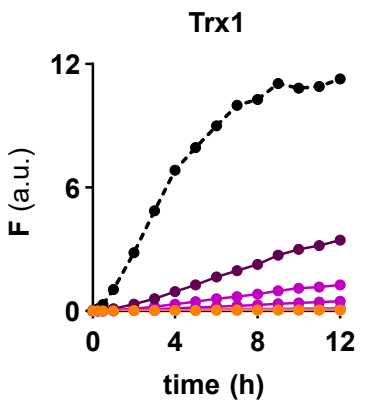

Grx1

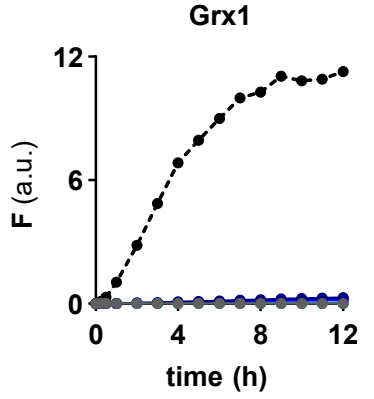

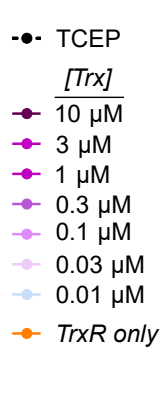

$\begin{aligned} & - \text { TCEP } \\ & {[\text { GrX] }} \\ \rightarrow & 10 \mu \mathrm{M} \\ \rightarrow & 3 \mu \mathrm{M} \\ \rightarrow & 1 \mu \mathrm{M} \\ \rightarrow & 0.3 \mu \mathrm{M} \\ \rightarrow & 0.1 \mu \mathrm{M} \\ \rightarrow & 0.03 \mu \mathrm{M} \\ \rightarrow & 0.01 \mu \mathrm{M} \\ \rightarrow & \text { GR }+ \text { GSH only }\end{aligned}$

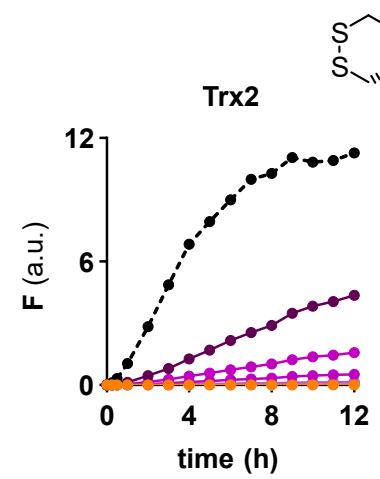

S'
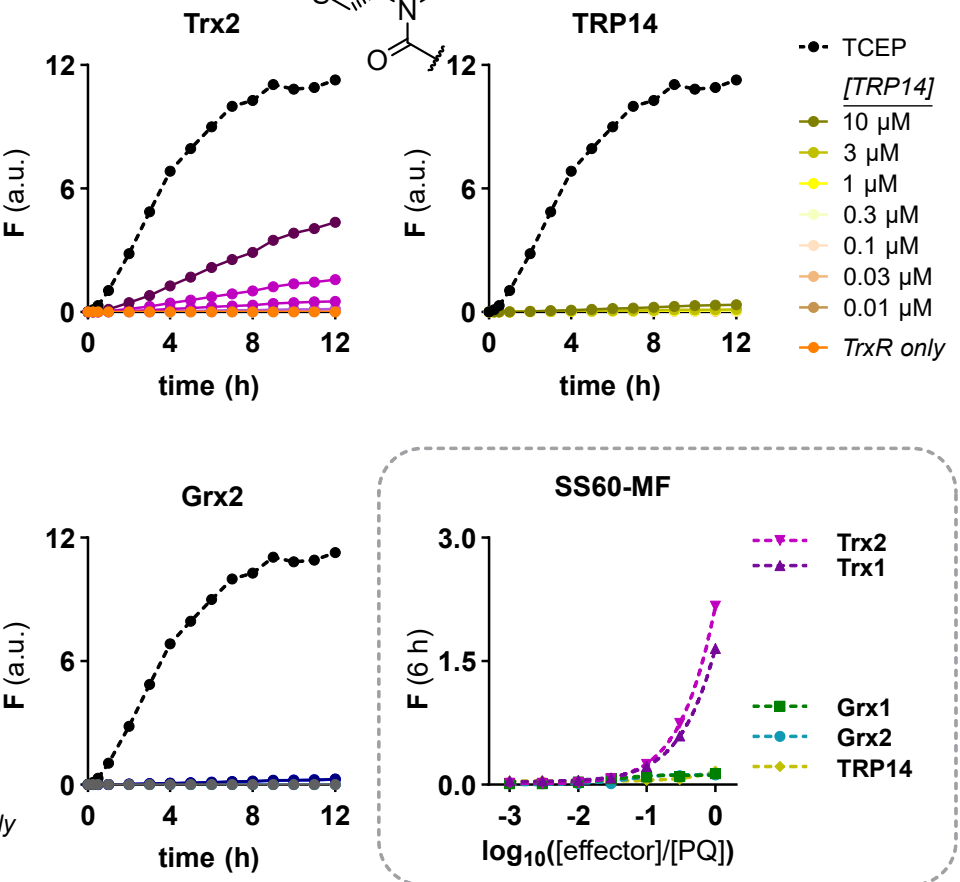

SS60-MF

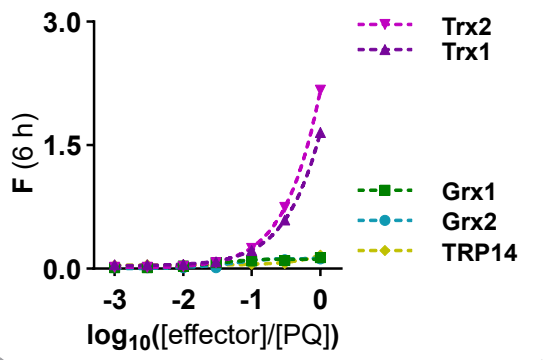

b

\section{SS00M-MF (10 $\mu \mathrm{M})$}

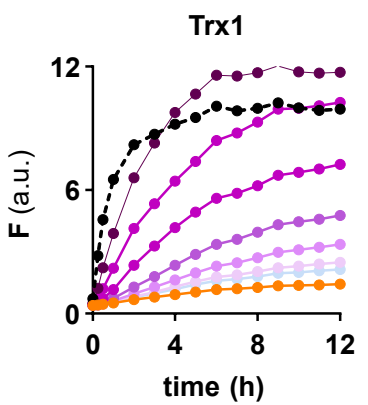

Grx1

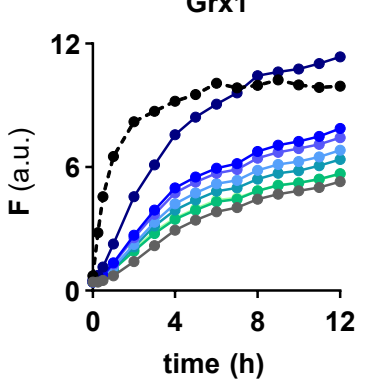

$$
\begin{aligned}
& \text { - - TCEP } \\
& \rightarrow \frac{[\operatorname{Tr} \mathrm{x}]}{10 \mu \mathrm{M}} \\
& \rightarrow 3 \mu \mathrm{M} \\
& \rightarrow 1 \mu \mathrm{M} \\
& \rightarrow 0.3 \mu \mathrm{M} \\
& -0.1 \mu \mathrm{M} \\
& 0.03 \mu \mathrm{M} \\
& -0.01 \mu \mathrm{M} \\
& \rightarrow \text { TrxR only }
\end{aligned}
$$

time (h)
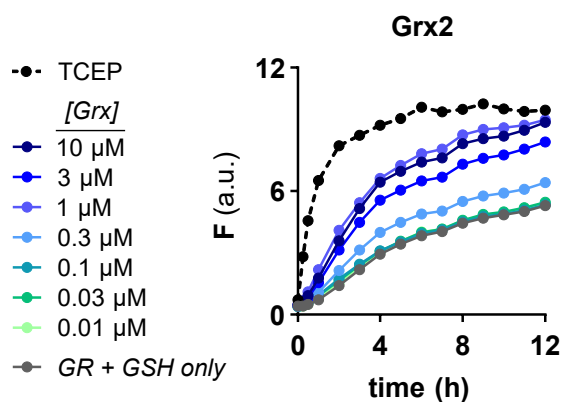
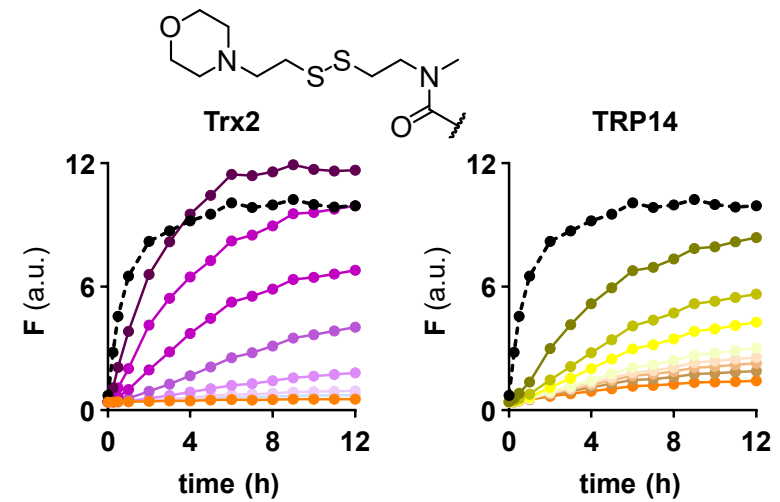

- TCEP

[TRP14]

$\rightarrow 3 \mu \mathrm{M}$

$1 \mu \mathrm{M}$

$0.3 \mu \mathrm{M}$

$0.1 \mu \mathrm{M}$

$-0.03 \mu \mathrm{M}$

$-0.01 \mu \mathrm{M}$

- TrxR only

Fig. S10 Redox effector titration assay: (a) Timecourse fluorescence intensity ( $t=0-6 \mathrm{~h}$ ) of SS60-MF (10 $\mu \mathrm{M})$ upon incubation with Trx1, Trx2, TRP14, Grx1 or Grx2 (0.01-10 $\mu \mathrm{M})$ as compared to a reference experiment: TCEP $(200 \mu \mathrm{M})$. All mixtures additionally containing $\beta$-NADPH $(100 \mu \mathrm{M})$ and the respective upstream system TrxR1, TrxR2 $(20 \mathrm{nM})$ or GR $(20 \mathrm{nM})+\mathrm{GSH}(100 \mu \mathrm{M})$. (b) Same evaluation of SS00m-MF $(10 \mu \mathrm{M})$. 

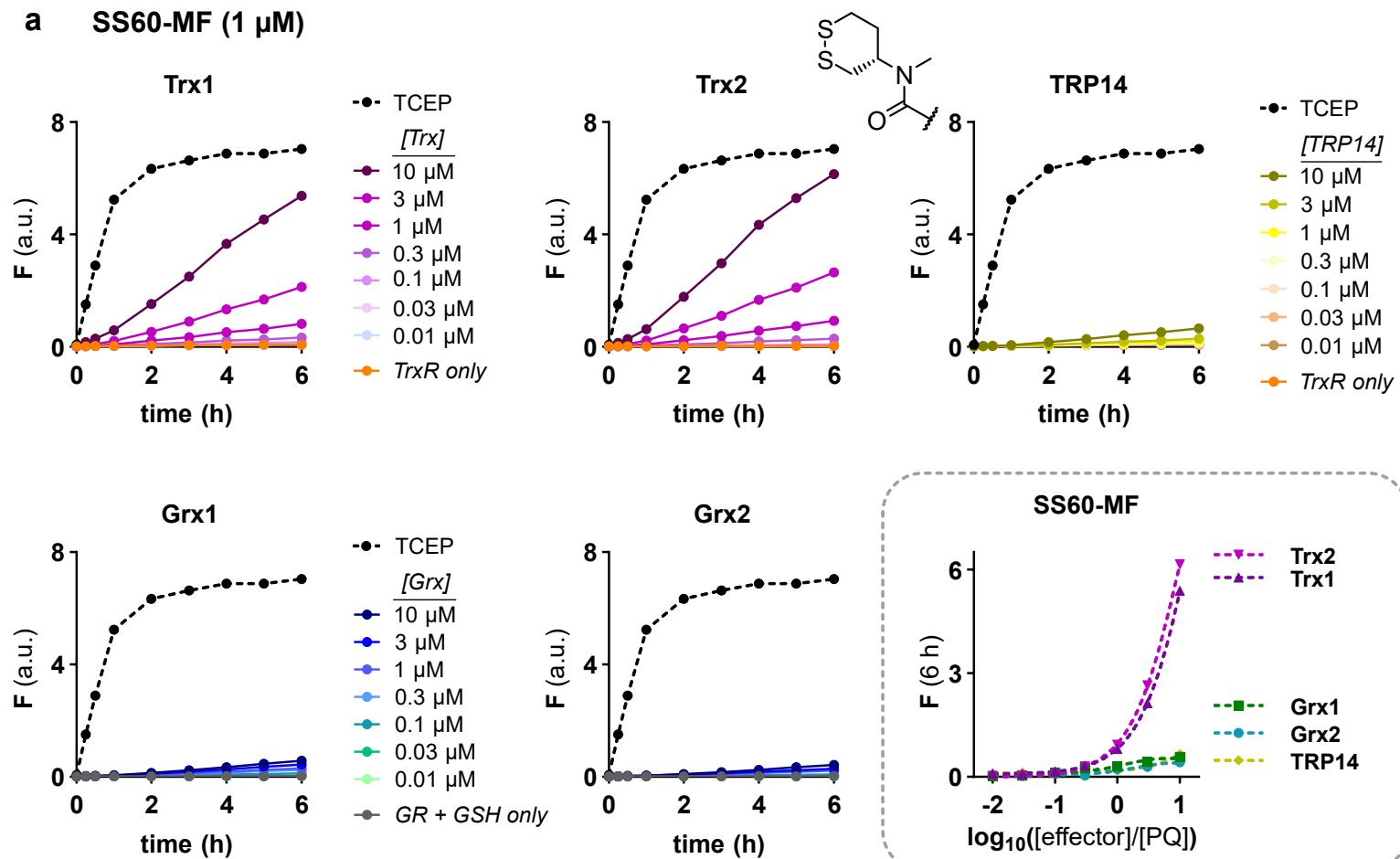

SS60-MF

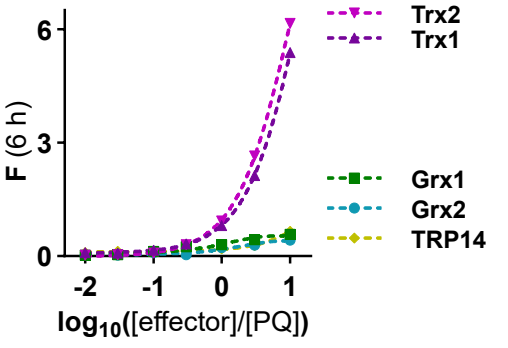

b

SS00M-MF (1 $\mu \mathrm{M})$
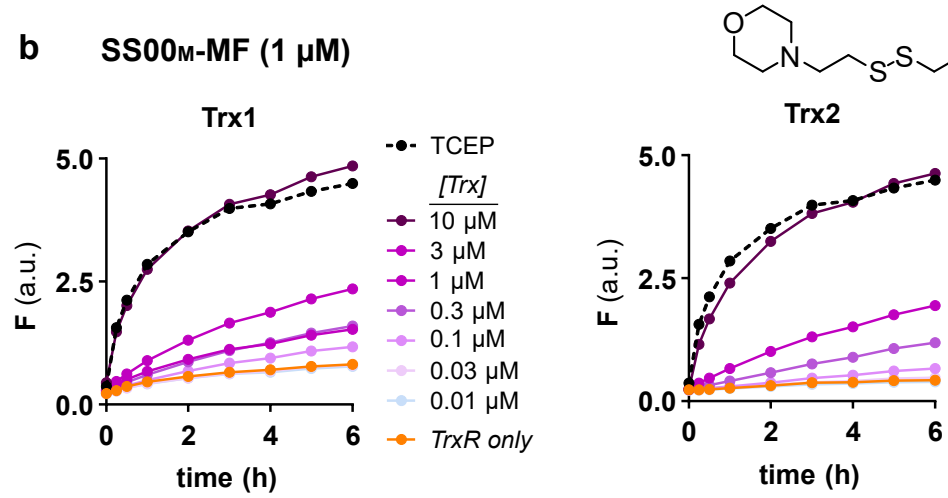

$\stackrel{\overbrace{}^{N}}{\overbrace{}^{\prime}}$

$\log _{10}([$ effector $] /[\mathrm{PQ}])$

Grx1

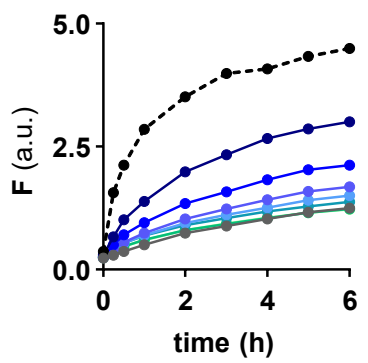

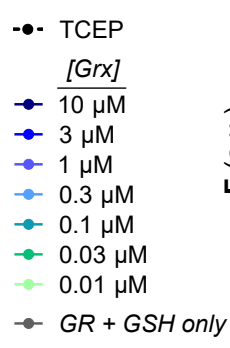

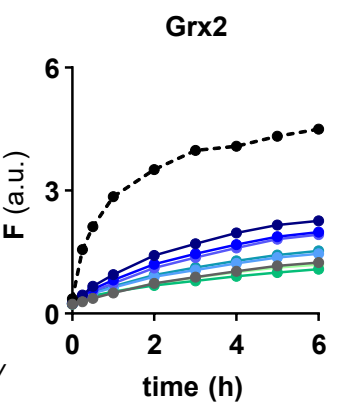

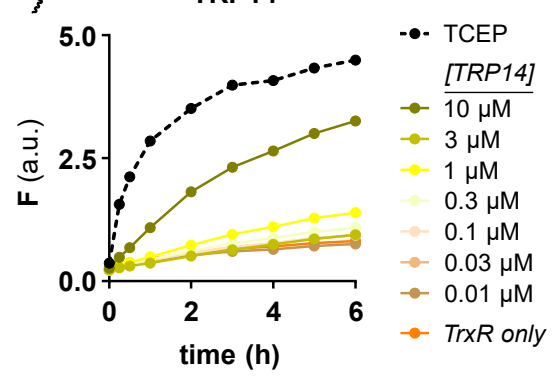

SSO0-MF

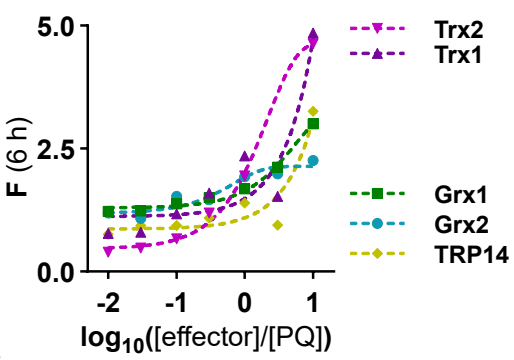

Fig. S11 Redox effector titration assay: (a) Timecourse fluorescence intensity ( $\mathrm{t}=0-6 \mathrm{~h}$ ) of SS60-MF (1 $\mu \mathrm{M})$ upon incubation with Trx1, Trx2, TRP14, Grx1 or Grx2 (0.01-10 $\mu \mathrm{M})$ as compared to a reference experiment: TCEP $(200 \mu \mathrm{M})$. All mixtures additionally containing $\beta$-NADPH $(100 \mu \mathrm{M})$ and the respective upstream system TrxR1, TrxR2 $(20 \mathrm{nM})$ or GR $(20 \mathrm{nM})+\mathrm{GSH}(100 \mu \mathrm{M})$. (b) Equal evaluation of SS00-MF $(1 \mu \mathrm{M})$. 

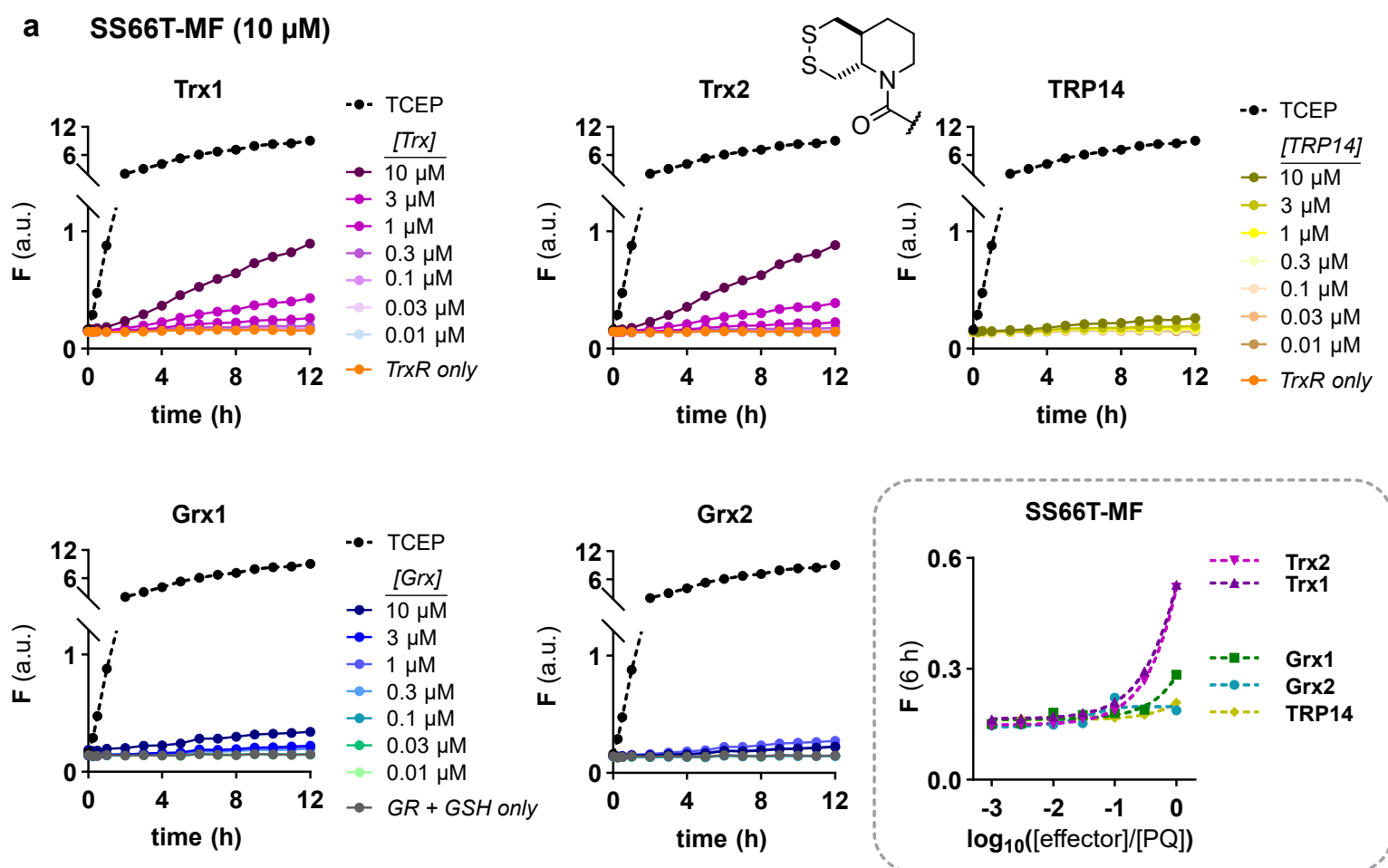

b SS66C-MF (10 $\mu \mathrm{M})$
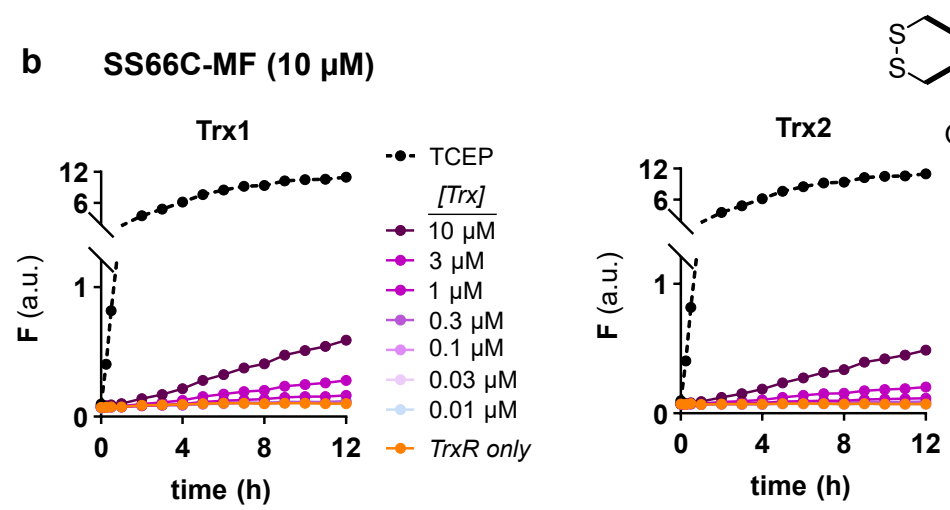<smiles>CC(C)C(=O)N1CCCC2CSCC21</smiles>
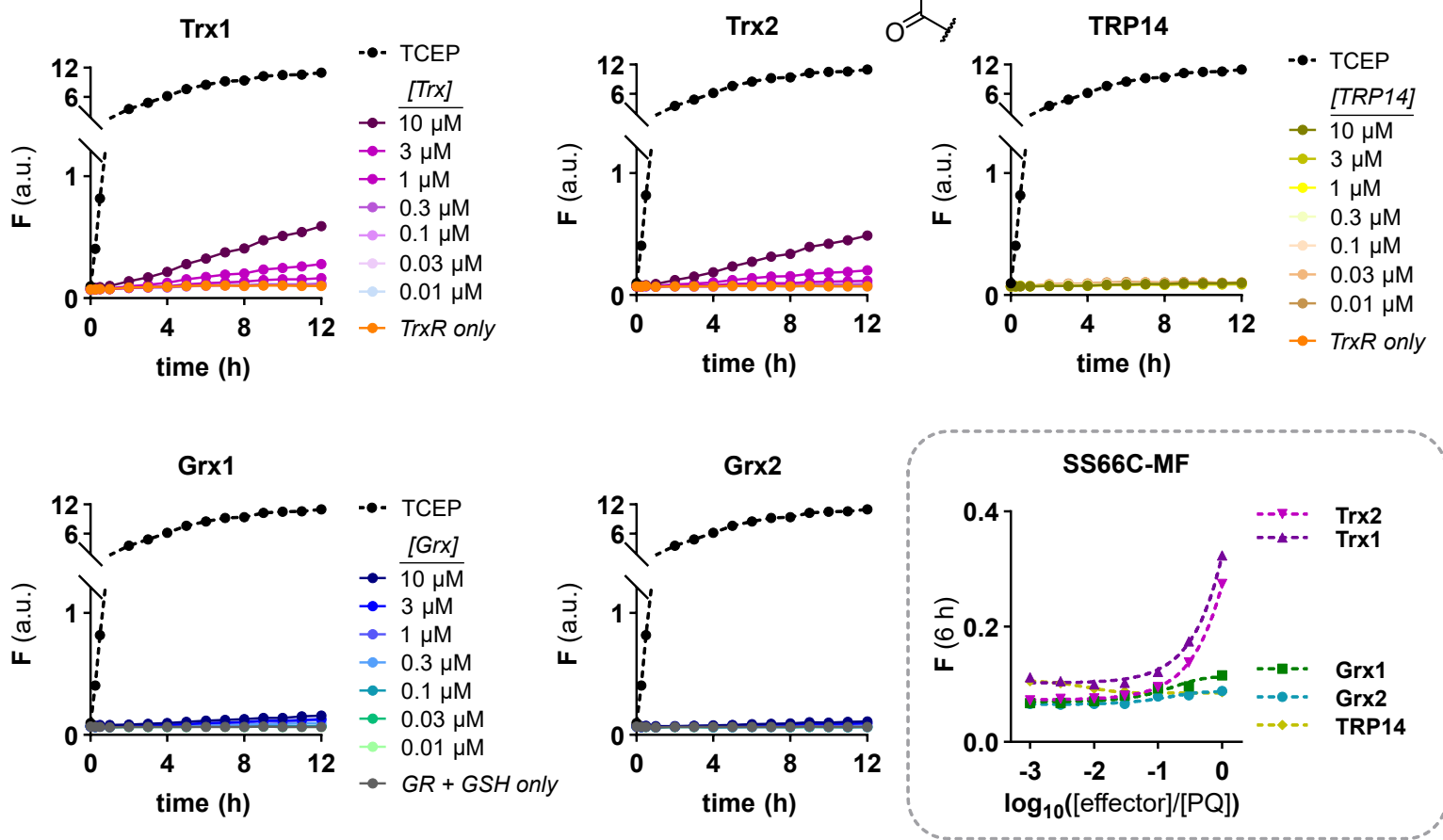

Fig. S12 Redox effector titration assay: (a) Timecourse fluorescence intensity $(t=0-6 \mathrm{~h})$ of SS66T-MF $(10 \mu \mathrm{M})$ upon incubation with Trx1, Trx2, TRP14, Grx1 or Grx2 (0.01-10 $\mu \mathrm{M})$ as compared to a reference experiment: TCEP $(200 \mu \mathrm{M})$. All mixtures additionally containing $\beta-\mathrm{NADPH}(100 \mu \mathrm{M})$ and the respective upstream system TrxR1, TrxR2 (20 nM) or GR (20 nM) + GSH (100 $\mu$ M). (b) Same evaluation of SS66C-MF $(10 \mu M)$. 

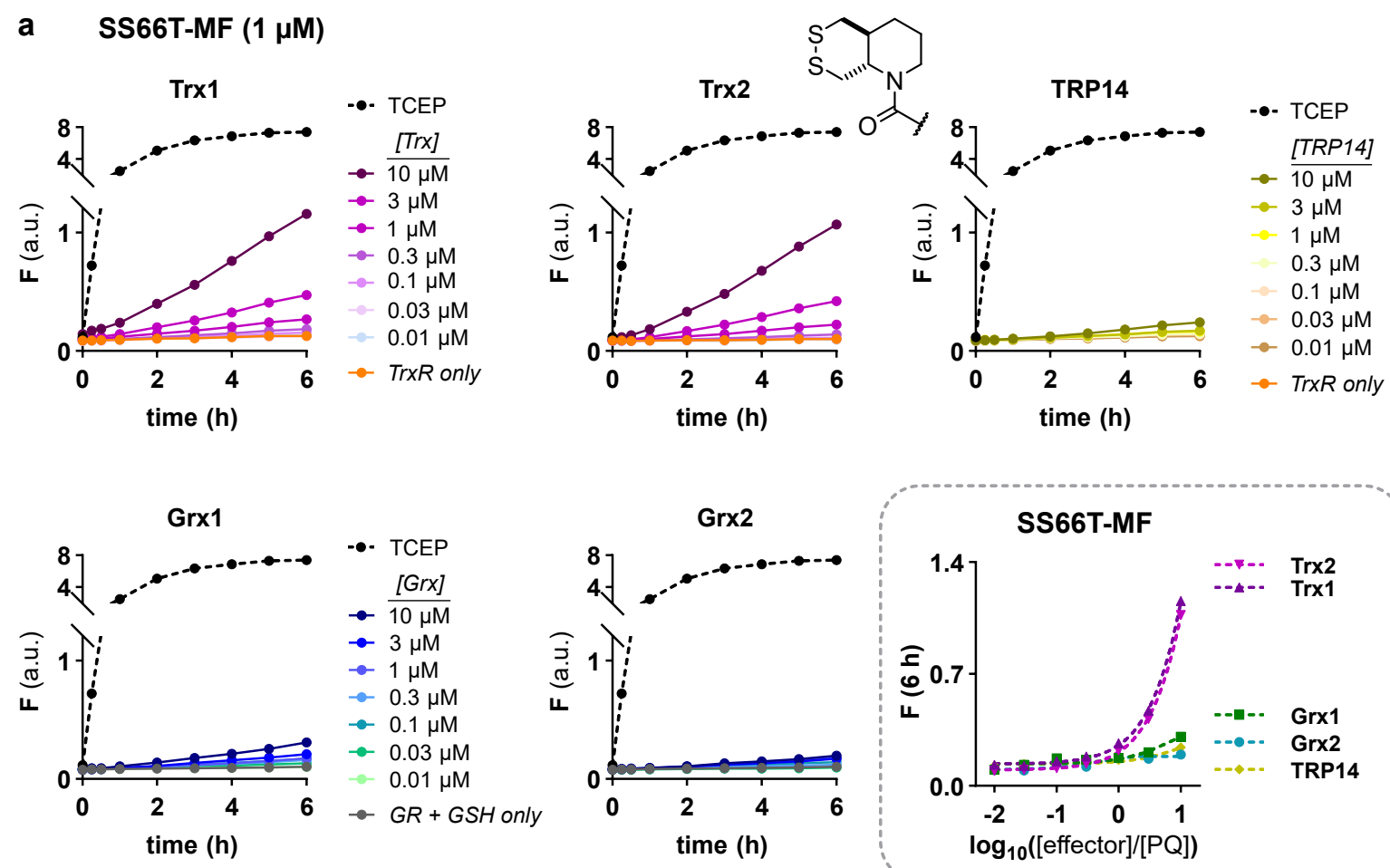

b

SS66C-MF (1 $\mu \mathrm{M})$
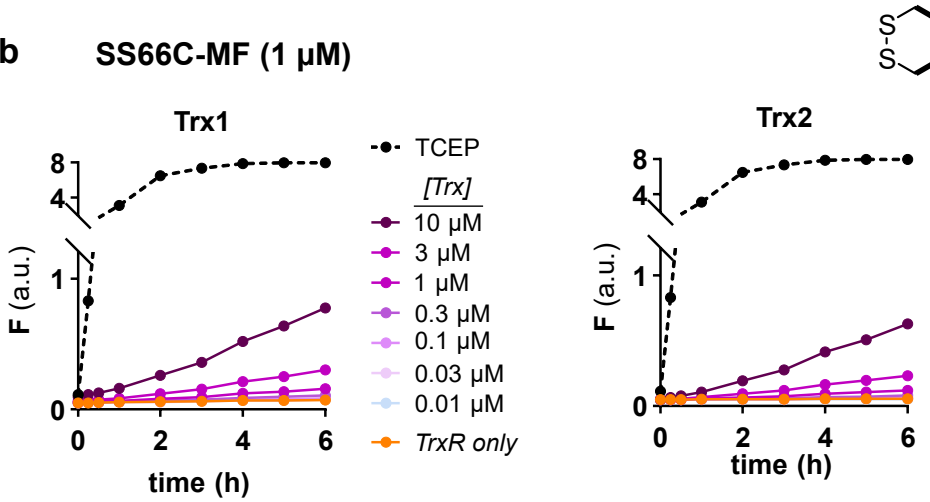<smiles>CCC(=O)N1CCCC2CSSCC21</smiles>
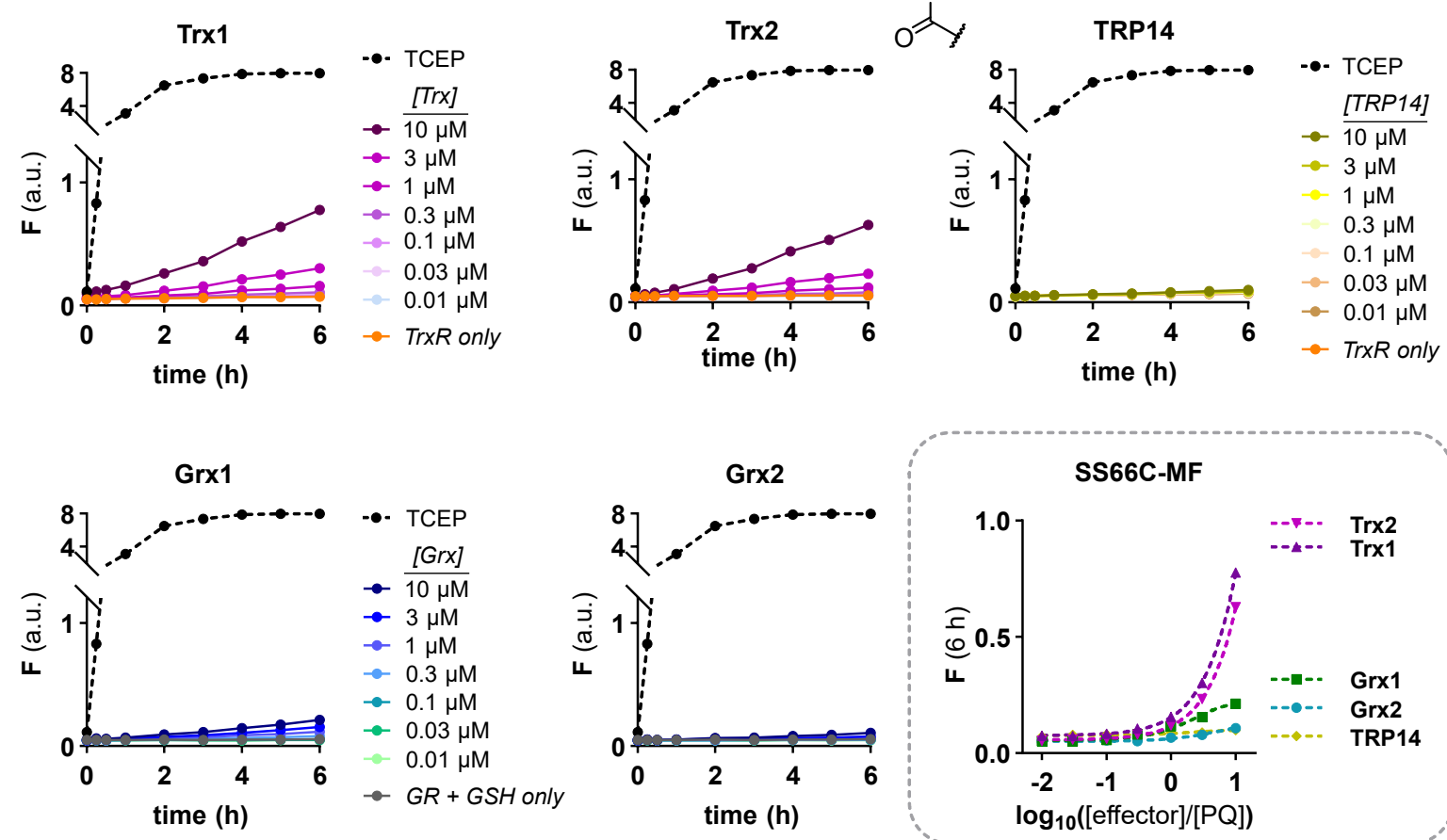

Fig. S13 Redox effector titration assay: (a) Timecourse fluorescence intensity ( $t=0-6 \mathrm{~h}$ ) of SS66T-MF ( $1 \mu \mathrm{M})$ upon incubation with Trx1, Trx2, TRP14, Grx1 or Grx2 (0.01-10 $\mu \mathrm{M})$ as compared to a reference experiment: TCEP $(200 \mu \mathrm{M})$. All mixtures additionally containing $\beta$-NADPH $(100 \mu \mathrm{M})$ and the respective upstream system TrxR1, TrxR2 $(20 \mathrm{nM})$ or GR $(20 \mathrm{nM})+\mathrm{GSH}(100 \mu \mathrm{M})$. (b) Same evaluation of SS66C-MF (1 $\mu \mathrm{M})$. 


\section{General methods}

\subsection{Enzyme biochemistry}

For early enzymatic activation profiling we used human natively isolated cytosolic thioredoxin 1 (hTrx 1$)$, native thioredoxin reductase 1 ( $\mathrm{hTrxR} 1$ ) natively isolated from human placenta, ${ }^{14}$ recombinant human thioredoxin reductase 1 point mutant (U498C mutant - hTrxRU498C) ${ }^{15}$ and recombinant human glutathione reductase $(\mathrm{hGR}) .^{16}$

For early redox effector titrations we used human recombinant thioredoxin 1 (Trx1) (lyophilized), recombinant human glutaredoxin 1 (Grx1) (lyophilized from $10 \mu \mathrm{L}$ TE-buffer, pH 7.5), human thioredoxin reductase (TrxR1) $(0.6 \mathrm{mg} / \mathrm{mL}$ in $50 \%$ glycerol/TE-buffer, $\mathrm{pH} 7.5)$ and baker's yeast glutathione reductase (GR) $(100 \mu \mathrm{M}$ in $50 \%$ glycerol/TE-buffer, $\mathrm{pH} 7.5)$ as obtained from IMCO Corp., Stockholm (Sweden).

For final redox effector titrations (including Fig 7, Fig S8-S13) we used recombinant human proteins expressed in E. coli: thioredoxin 1 (Trx1) in $50 \mathrm{mM}$ Tris, $2 \mathrm{mM}$ EDTA, pH 7.5, recombinant thioredoxin 2 (Trx2) in $100 \mathrm{mM}$ Tris, $500 \mathrm{mM} \mathrm{NaCl}, 2 \mathrm{mM}$ EDTA, 30\% glycerol, $\mathrm{pH}$ 7.5, thioredoxin-related protein 14 (TRP14) in $100 \mathrm{mM}$ Tris, $300 \mathrm{mM} \mathrm{NaCl}, 2 \mathrm{mM}$ EDTA, 30\% glycerol, pH 7.5, glutaredoxin 1 (Grx1) in TES buffer, 30\% glycerol, pH 7.5 and recombinant human glutaredoxin 2 (Grx2) in $100 \mathrm{mM}$ Tris, $2 \mathrm{mM}$ EDTA, pH 7.5, enabled by the upstream reductases selected from thioredoxin reductase 1 (TrxR1) in $100 \mathrm{mM}$ Tris, $2 \mathrm{mM}$ EDTA, 50\% glycerol, $\mathrm{pH}$ 7.5, thioredoxin reductase 2 (TrxR2) in in $100 \mathrm{mM}$ Tris, $2 \mathrm{mM}$ EDTA, $50 \%$ glycerol, pH 7.5 or glutathione reductase (GR) in in $50 \mathrm{mM}$ Tris, $100 \mathrm{mM} \mathrm{NaCl} 2 \mathrm{mM}$ EDTA, $50 \%$ glycerol, pH 7.5 .

\subsection{Fluorescence spectroscopy and detection}

Fluorescence spectroscopy measurements were conducted using a Varian Cary Eclipse fluorescence spectrophotometer from Agilent Technologies Inc., Santa Clara (USA) at $298 \mathrm{~K}$ either using a constant excitation mode (e.g. ex $355 \mathrm{~nm}$ ) with emission fluorescence intensity screening or alternatively a constant emission mode (e.g. em $520 \mathrm{~nm}$ ) with excitation efficiency screening mode. For exemplary timecourse measurements of emission spectra a constant excitation mode ( $355 \mathrm{~nm}$ for SS60-PQ and $485 \mathrm{~nm}$ for SS50-MF) was used.

Raw fluorescence readout of cell-free activity was performed either using a FluoStar Omega plate reader from BMG Labtech, Ortenburg (Germany), a Tecan Infinite M200Pro plate reader from Tecan, Maennedorf, Switzerland, set to exc: $355 \mathrm{bp} 10$ and em: $5201 \mathrm{p}$ or to ex: $485 \mathrm{bp} 10$ and em: $5201 \mathrm{p}$ recording fluorescence intensity.

\subsection{Laboratory techniques}

All solvents, reagents and building blocks were purchased from standard commercial sources. Anhydrous solvents obtained in septum-capped bottles and analytical grad or higher quality solvents were used without purification. Industrial grade solvents were distilled prior to use. Unless otherwise stated, reactions were performed at room temperature without precautions regarding air or moisture and were stirred using a magnetic Teflon ${ }^{\circledR}$-coated stir bars. Air or moisture sensitive reactions were conducted in dry Schlenk glassware.

Flash column chromatography (FCC) was performed on Geduran ${ }^{\circledR}$ Si 60 silica gel from Merck GmbH, Darmstadt (Germany) an optionally conducted using a Biotage ${ }^{\circledR}$ Select automated column chromatography system from Biotage $\mathrm{GmbH}$, Uppsala (Sweden). Unless stated otherwise, and thin layer chromatography (TLC) to monitor reactions and determine $R_{f}$ values was performed on silica coated aluminium sheets with fluorescent indicator (TLC Silica gel 60 F254 from Merck GmbH, Darmstadt, Germany) with visualisation by UV irradiation $(254 \mathrm{~nm} / 360 \mathrm{~nm})$ or staining with $\mathrm{KMnO}_{4}$ solution (3.0 $\mathrm{g} \mathrm{KMnO}_{4}, 20 \mathrm{~g} \mathrm{~K}_{2} \mathrm{CO}_{3}, 0.30 \mathrm{~g} \mathrm{KOH}, 0.30 \mathrm{~L} \mathrm{H}_{2} \mathrm{O}$ ).

\subsection{Analytical methods}

High resolution mass spectrometry (HRMS) was conducted either using a Thermo Finnigan LTQ FT Ultra FourierTransform ion cyclotron resonance spectrometer from ThermoFisher Scientific GmbH, Dreieich (Germany) applying electron spray ionisation (ESI) with a spray capillary voltage of $4 \mathrm{kV}$ at temperature $250^{\circ} \mathrm{C}$ with a method dependent range from 50 to $2000 \mathrm{u}$ or a Finnigan MAT 95 from Thermo Fisher Scientific, Dreieich (Germany) applying electron ionisation (EI) at a source temperature 
of $250{ }^{\circ} \mathrm{C}$ and an electron energy of $70 \mathrm{eV}$ with a method dependent range from 40 to $1040 \mathrm{u}$. All reported $\mathrm{m} / \mathrm{z}$ values refer to positive ionization mode, unless stated otherwise.

Nuclear magnetic resonance (NMR) spectroscopy was performed using a Bruker Avance (600/150 MHz, with TCl cryoprobe) or a Bruker Avance III HD Biospin $(400 / 100 \mathrm{MHz}$, with BBFO cryoprobeTM) from Bruker Corp., Billerica (USA) either at $400 \mathrm{MHz}$ or $500 \mathrm{MHz}$. ${ }^{19} \mathrm{~F}$-NMR spectra were recorded on a Bruker Avance III spectrometer (400 MHz for ${ }^{1} \mathrm{H} ; 377 \mathrm{MHz}$ for $\left.{ }^{19} \mathrm{~F}\right)$. NMR-spectra were measured at $298 \mathrm{~K}$, unless stated otherwise, and were analysed with the program MestreNova 12 developed by MestreLab Ltd., Santiago de Compostela (Spain). ${ }^{1} \mathrm{H}-\mathrm{NMR}$ spectra chemical shifts $(\delta)$ in parts per million (ppm) relative to tetramethylsilane $(\delta=0 \mathrm{ppm})$ are reported using the residual protic solvent $\left(\mathrm{CHCl}_{3}\right.$ in $\mathrm{CDCl}_{3}: \delta=7.26 \mathrm{ppm}$, DMSO-d $\mathrm{d}_{5}$ in DMSO-d 6 : $\delta=2.50 \mathrm{ppm}, \mathrm{CHD}_{2} \mathrm{OD}$ in $\mathrm{CD}_{3} \mathrm{OD}$ : $\delta=3.31 \mathrm{ppm}$ ) as an internal reference. For ${ }^{13} \mathrm{C}$-NMR spectra, chemical shifts in ppm relative to tetramethylsilane $(\delta=0 \mathrm{ppm})$ are reported using the central resonance of the solvent signal $\left(\mathrm{CDCl}_{3}\right.$ : $\delta=77.16 \mathrm{ppm}$, DMSO-d 6 : $\delta=39.52 \mathrm{ppm}, \mathrm{CD}_{3} \mathrm{OD}: \delta=49.00 \mathrm{ppm}$ ) as an internal reference. For ${ }^{1} \mathrm{H}-\mathrm{NMR}$ spectra in addition to the chemical shift the following data is reported in parenthesis: multiplicity, coupling constant(s), number of hydrogen atoms and, if available, assignment. The abbreviations for multiplicities and related descriptors are $s=$ singlet, $d=$ doublet, $t=$ triplet, $q=$ quartet, $\mathrm{p}=$ pentuplet, hept $=$ heptet or combinations thereof, $\mathrm{m}=$ multiplet and $\mathrm{br}=$ broad. The numbering scheme used for the assignments is specified in each case in a figure depicting the respective molecular structure and does not follow any convention. The reported assignments are supported by 2D-NMR experiments (HMBC, HSQC, COSY). Where known products matched literature analysis data, only selected data acquired are reported.

Analytical high pressure liquid chromatography (HPLC) analysis was conducted either using an Agilent 1100 system from Agilent Technologies Corp., Santa Clara (USA) equipped with a DAD detector and a Hypersil Gold HPLC column from ThermoFisher Scientific GmbH, Dreieich (Germany) or a Agilent 1200 SL system Agilent Technologies Corp., Santa Clara (USA) equipped with a DAD detector, a Hypersil Gold HPLC column from ThermoFisher Scientific GmbH, Dreieich (Germany) and consecutive low-resolution mass detection using a LC/MSD IQ mass spectrometer applying ESI from Agilent Technologies Corp., Santa Clara (USA). For both systems mixtures of water (analytical grade, $0.1 \%$ formic acid) and MeCN (analytical grade, $0.1 \%$ formic acid) were used as eluent systems.

Single crystal X-Ray crystallography was conducted using a Bruker D8 Venture TXS system from Bruker Corp., Billerica (USA) equipped with a multilayer mirror monochromator and a Mo Ka rotating anode X-ray tube $(\lambda=0.71073 \AA)$. The frames were integrated with the Bruker SAINT software package (Bruker, 2012. SAINT. Bruker AXS Inc., Madison, Wisconsing, USA). Data were corrected for absorption effects using the Multi-Scan method (SADABS) (Sheldrick, G. M., 1996. SADABS. University of Goettingen, Germany). The structures were solved and refined using the Bruker SHELXTL Software Package. ${ }^{17}$ The figures have been drawn at the $50 \%$ ellipsoid probability level. ${ }^{18}$ Crystallographic data have been deposited with the Cambridge Crystallographic Data Centre, CCDC, 12 Union Road, Cambridge CB21EZ, UK. Copies of the data can be obtained free of charge on quoting the depository numbers CCDC 2072440 (5), 2072441 (66), 2072442 (65), 2072438 (67), 2072439 (75). (https://www.ccdc.cam.ac.uk/structures/). 


\section{Synthetic Procedures}

\subsection{General protocols}

General protocol A: Amine protection with di-tert-butyl dicarboxylate. (N-Boc protection)

$$
\underset{\mathrm{H}}{\stackrel{\mathrm{Boc}_{2} \mathrm{O}, \mathrm{NEt}_{3}}{\text { dioxane/ } \mathrm{H}_{2} \mathrm{O} \text {, r.t. }}}
$$

To a $0.25-0.5 \mathrm{M}$ solution of the primary or secondary amine (1.0 eq.) in a mixture of dioxane/water (1:1) was added $\mathrm{NEt}_{3}$ (1.3 eq per amino group) and a $0.5 \mathrm{M}$ solution of di-tert-butyl dicarbonate (1.0 eq. per amino group) in 1,4-dioxane and the resulting biphasic solution was stirred at r.t. for $15 \mathrm{~h}$. The reaction mixture was acidified to $\mathrm{pH}<4$ with $2 \mathrm{M}$ aq. $\mathrm{HCl}$ and diluted with EtOAc. The organic phase was separated, washed with sat. aq. $\mathrm{NaCl}$ and the combined aqueous layers were extracted with EtOAc (3x). The combined organic layers were dried over $\mathrm{Na}_{2} \mathrm{SO}_{4}$ and concentrated to afford the desired $\mathrm{N}$-Boc protected amine. If necessary, the crude product was further purified by FCC or recrystallization. ${ }^{19,20}$

General protocol B: Reduction of dicarboxylic acid diesters with $\mathrm{LiAlH}_{4}$.<smiles>CCC1OC(=O)N(C(C)C)C1COC</smiles><smiles>CCOC(=O)OCC</smiles>

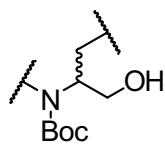

To a $0.2 \mathrm{M}$ suspension of $\mathrm{LiAlH}_{4}$ (3.0 eq.) in anhydrous $\mathrm{Et}_{2} \mathrm{O}$ or THF under nitrogen atmosphere at $0{ }^{\circ} \mathrm{C}$ was dropwise added a $0.2 \mathrm{M}$ solution of dimethyl dicarboxylate $\left(1.0\right.$ eq.) in anhydrous $\mathrm{Et}_{2} \mathrm{O}$ with vigorous stirring. The reaction mixture was stirred for $30 \mathrm{~min}$ at $0{ }^{\circ} \mathrm{C}$, was then allowed to warm to r.t. and was further stirred at r.t. for $1 \mathrm{~h}$, before water $(1 \mathrm{~mL}$ per $1 \mathrm{mmol}$ substrate) was carefully added at $0{ }^{\circ} \mathrm{C}$. This was followed by addition of $2 \mathrm{M}$ aq. $\mathrm{NaOH}$ until the grey suspension cleared and a colourless solid formed. Then, $\mathrm{Na}_{2} \mathrm{SO}_{4}(20 \mathrm{~g}$ per $1 \mathrm{mmol}$ substrate) was added, the mixture was filtered and the solids were washed with EtOAc. The filtrate was concentrated under reduced pressure to afford the desired product, which was used without further purification.

General protocol C: Thioacetate formation by Mitsunobu reaction.<smiles>CC(C)[N+]([O-])=CC1CCCOC1</smiles>

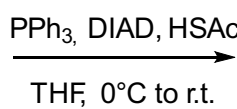

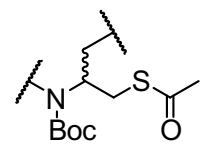

To $\mathrm{PPh}_{3}$ (1.2 eq. per hydroxy group) in anhydrous THF $(0.25 \mathrm{M})$ under nitrogen atmosphere was dropwise added DIAD (1.25 eq. per hydroxy group) at $0{ }^{\circ} \mathrm{C}$. After the reaction mixture had stirred for 30 min, a $0.3 \mathrm{M}$ solution of the alcohol or diol (1.0 eq.) in anhydrous THF and successively neat HSAc (1.25 eq. per hydroxy group) were added dropwise was added dropwise and immediate change of colour was observed. The reaction mixture was further stirred at $0{ }^{\circ} \mathrm{C}$ for $1 \mathrm{~h}$, was then allowed to warm to r.t. and was further stirred for $15 \mathrm{~h}$. All volatiles were removed under reduced pressure and the residue was purified by FCC.

General protocol D: Monomethylation of N-Boc protected amines.

$$
\sim_{\mathrm{H}}^{\mathrm{Boc}} \underset{\mathrm{DMF}, 0^{\circ} \mathrm{C} \text { to r.t. }}{\stackrel{\mathrm{NaH}, \mathrm{Mel}}{\longrightarrow}}
$$

To a $0.02 \mathrm{M}$ solution of the $\mathrm{N}$-Boc amine (1.0 eq.) in anhydrous DMF under nitrogen atmosphere at $0{ }^{\circ} \mathrm{C}$ was portionwise added $60 \% \mathrm{NaH}$ dispersion in mineral oil (1.1 eq. per amino group), followed by iodomethane (1.2 eq. per amino group). The reaction mixture was stirred at $0^{\circ} \mathrm{C}$ for $30 \mathrm{~min}$, was then allowed to warm to r.t. and stirred until complete reaction turnover was confirmed by thin layer chromatography. The mixture was diluted with EtOAc and washed with sat. aq. $\mathrm{NaCl}(2 \times)$. The combined aqueous layers were extracted with EtOAc $(3 x)$ and the combined organic layers were dried over $\mathrm{Na}_{2} \mathrm{SO}_{4}$. filtered and concentrated under reduced pressure to afford the crude product as a colourless oil/solid, which was further purified by FCC. ${ }^{21}$ 
General protocol E: Thioester cleavage and dithiol oxidation.

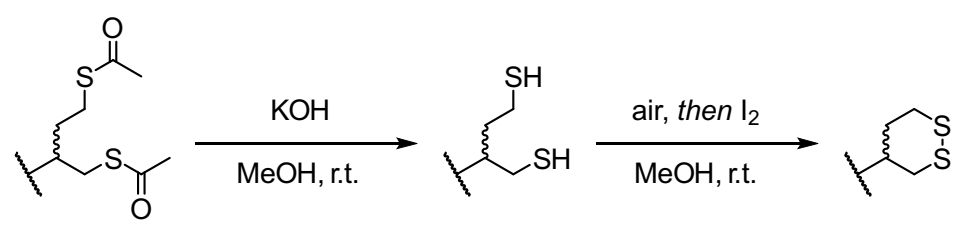

The (bis-)ethanthioate (1.0 eq.) was dissolved in a $0.17 \mathrm{M}$ methanolic $\mathrm{KOH}$ solution (4.0 eq.) and mixture was stirred open to air for $15 \mathrm{~h}$. Then, the mixture was concentrated under reduced pressure, diluted with water and extracted with EtOAc (3x). The combined organic layers were dried over $\mathrm{Na}_{2} \mathrm{SO}_{4}$ and concentrated under reduced pressure to afford the desired product, which was used without further purification. In case of incomplete oxidation of the intermediate thiol/dithiol, a $0.5 \mathrm{M}$ solution of $\mathrm{I}_{2}$ in $\mathrm{MeOH}$ was added dropwise until a permanently coloured solution was observed, before the reaction mixture was concentrated. Dilute aq. $\mathrm{Na}_{2} \mathrm{~S}_{2} \mathrm{O}_{3}$ was used to quench excess of $\mathrm{I}_{2}$ during the aq. workup. ${ }^{22}$

General protocol F: Amine deprotection.

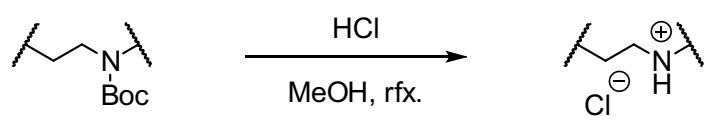

To a $0.05 \mathrm{M}$ solution of the $\mathrm{N}$-Boc amine (1.0 eq.) in anhydrous $\mathrm{MeOH}$ was added a $1.25 \mathrm{M}$ methanolic solution of $\mathrm{HCl}(10$ eq.) and the mixture was heated to reflux for $2 \mathrm{~h}$. Then, all volatiles were removed under reduced pressure and the remaining hydrochloride salt was used without further purification.

General protocol G: Chloroformate formation with triphosgene and carbamate coupling.

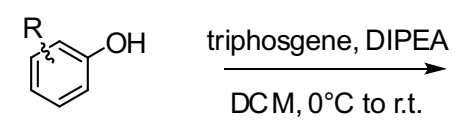<smiles>O=C(Cl)Oc1ccccc1</smiles>

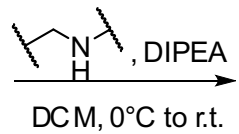<smiles>[R]c1cccc(OC(=O)N(CC)CC(C)C)c1</smiles>

Step 1: To a $0.04 \mathrm{M}$ solution of the phenol (1.0 eq.) in anhydrous DCM under nitrogen atmosphere at $0{ }^{\circ} \mathrm{C}$ was added a $0.2 \mathrm{M}$ solution of triphosgene (BTC) (1.2 eq.) in anhydrous DCM, followed by a $0.1 \mathrm{M}$ solution of DIPEA (4 eq.) in anhydrous DCM. The resulting mixture was stirred at $0{ }^{\circ} \mathrm{C}$ for $30 \mathrm{~min}$, was then allowed to warm to r.t. and was further stirred for $1.5 \mathrm{~h}$. All volatiles were evaporated by applying high vacuum to afford the corresponding chloroformate derivative was redissolved in anhydrous DCM to obtain a $0.015 \mathrm{M}$ solution of the corresponding chloroformate intermediate (assuming quantitative conversion of the phenol substrate). The volatiles were collected in a liquid nitrogen trap and residual phosgene was discharged by subsequently adding a $2 \mathrm{M}$ aq. $\mathrm{NaOH}$ and piperidine.

Step 2: To a $0.1 \mathrm{M}$ suspension of the secondary amine or its hydrochloride salt (1.0 eq.) in anhydrous DCM was added DIPEA (1.2 eq.). The resulting solution was added dropwise at $0{ }^{\circ} \mathrm{C}$ to the $0.015 \mathrm{M}$ chloroformate solution ( 1.0 eq.) obtained in step 1 and the mixture was stirred at $0{ }^{\circ} \mathrm{C}$ for 15 min before additional DIPEA (1.0 eq.) was added, then mixture was then allowed to warm to r.t. and was further stirred for $1.5 \mathrm{~h}$. After stirring at r.t. for $45 \mathrm{~min}$, sat. aq. $\mathrm{NaHCO}_{3}$ was added. The mixture was diluted with DCM and the layers were separated. The organic layer was washed with sat. aq. $\mathrm{NaCl}$ and the combined aq. layers were extracted with DCM $(2 \times)$. The combined organic layers were dried over $\mathrm{Na}_{2} \mathrm{SO}_{4}$, filtered and concentrated under reduced pressure to afford the crude product as a coloured solid. The residue was purified by FCC.

General protocol H: Amide coupling with benzoyl chloride.<smiles>O=C(Cl)c1ccccc1</smiles>

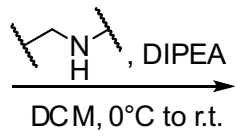<smiles>CC(C)CN(C(=O)c1ccccc1)C(C)C</smiles>

To a $0.1 \mathrm{M}$ suspension of the secondary amine or its hydrochloride (1.0 eq.) in anhydrous DCM were added $\mathrm{NEt}_{3}(2.0$ eq.) and benzoyl chloride (2.0 eq.). The mixture was stirred for $2 \mathrm{~h}$, concentrated under reduced pressure and the residue was purified by FCC. 


\subsection{Trigger synthesis}

\section{Annelated SS66-type precursor}

\section{dimethyl cis-piperidine-2,3-dicarboxylate (13)}

To a solution of dimethyl pyridine-2,3-dicarboxylate 1 (8.00 g, $41.0 \mathrm{mmol}$ ) in $\mathrm{MeOH}\left(35 \mathrm{~mL}\right.$ ) was added $96 \% \mathrm{H}_{2} \mathrm{SO}_{4}$ $(0.22 \mathrm{~mL}, 4.1 \mathrm{mmol})$ and $10 \% \mathrm{Pd} / \mathrm{C}(250 \mathrm{mg}, 0.235 \mathrm{mmol})$. The mixture was stirred under hydrogen atmosphere (40 bar) for $15 \mathrm{~h}$, filtered over Celite ${ }^{\circledR}$ and concentrated to afford $13\left(8.09 \mathrm{~g}, 40.2 \mathrm{mmol} 98 \%\right.$ ) as a colourless oil. ${ }^{23}$

TLC $\mathrm{R}_{f}=0.50$ (DCM:MeOH, 50:1). HRMS (ESI): $\mathrm{C}_{9} \mathrm{H}_{16} \mathrm{NO}_{4}{ }^{+}:[\mathrm{M}+\mathrm{H}]^{+}$calc. 202.10738, found 202.10734.

${ }^{1} \mathrm{H}-N M R\left(400 \mathrm{MHz}, \mathrm{CDCl}_{3}\right): \delta(\mathrm{ppm})=3.73(\mathrm{~s}, 3 \mathrm{H}), 3.68(\mathrm{~s}, 3 \mathrm{H}), 3.63(\mathrm{~d}, J=3.5 \mathrm{~Hz}, 1 \mathrm{H}), 3.10-2.95(\mathrm{~m}, 2 \mathrm{H})$, $2.76-2.62(\mathrm{~m}, 1 \mathrm{H}), 2.22-2.11(\mathrm{~m}, 1 \mathrm{H}), 2.07(\mathrm{~s}, 1 \mathrm{H}), 1.84-1.71(\mathrm{~m}, 1 \mathrm{H}), 1.61-1.41(\mathrm{~m}, 2 \mathrm{H}) \cdot{ }^{13} \mathrm{C}-\mathrm{NMR}(101 \mathrm{MHz}$, $\left.\mathrm{CDCl}_{3}\right) \delta(\mathrm{ppm})=173.3(\mathrm{C}=\mathrm{O}), 172.6(\mathrm{C}=\mathrm{O}), 59.5(\mathrm{CH}), 52.2\left(\mathrm{CH}_{3}\right), 52.0\left(\mathrm{CH}_{3}\right), 45.4\left(\mathrm{CH}_{2}\right), 42.3(\mathrm{CH}), 26.1\left(\mathrm{CH}_{2}\right)$, $23.5\left(\mathrm{CH}_{2}\right)$.

\section{dimethyl cis-N-Boc-piperidine-2,3-dicarboxylate (2)}<smiles>[2H]C([2H])(C)OC(=O)N1CCC[C@@H](C(=O)OC)C1(C(=O)OC)C(=O)OC</smiles>

Prepared from $13(3.00 \mathrm{~g}, 14.9 \mathrm{mmol})$ according to general protocol A. Compound 2 (4.43 g, $14.7 \mathrm{mmol}, 99 \%)$ was obtained as a colourless solid after FCC (isohexane/EtOAc).

TLC $\mathrm{R}_{f}=0.35$ (isohexane:EtOAc, 2:1). HRMS (ESI $\left.{ }^{+}\right): \mathrm{C}_{14} \mathrm{H}_{23} \mathrm{NNaO}_{6}{ }^{+}[\mathrm{M}+\mathrm{Na}]^{+}$: calc. 324.14176, found 324.14165. Individual rotamers were observed by NMR spectroscopy at $298 \mathrm{~K}$ and time-averaged spectra were obtained at $373 \mathrm{~K}$.

${ }^{1} \mathrm{H}-\mathrm{NMR}\left(400 \mathrm{MHz}\right.$, tetrachlorethane- $\left.d_{2}, 373 \mathrm{~K}\right): \delta(\mathrm{ppm})=5.32(\mathrm{~s}, 1 \mathrm{H}, 1-\mathrm{H}), 4.05-3.87\left(\mathrm{~m}, 1 \mathrm{H}, 5-\mathrm{H}_{\mathrm{A}}\right), 3.68(\mathrm{~s}$, $3 \mathrm{H}, 8-\mathrm{H}), 3.66(\mathrm{~s}, 3 \mathrm{H}, 9-\mathrm{H}), 2.85-2.71\left(\mathrm{~m}, 1 \mathrm{H}, 5-\mathrm{H}_{\mathrm{B}}\right), 2.59(\mathrm{ddd}, J=12.7,5.0,3.6 \mathrm{~Hz}, 1 \mathrm{H}, 2-\mathrm{H}), 2.11-1.92(\mathrm{~m}$, $\left.1 \mathrm{H}, 3-\mathrm{H}_{\mathrm{A}}\right), 1.74-1.66\left(\mathrm{~m}, 1 \mathrm{H}, 4-\mathrm{H}_{\mathrm{A}}\right), 1.61\left(\mathrm{td}, J=13.3,3.9 \mathrm{~Hz}, 1 \mathrm{H}, 3-\mathrm{H}_{\mathrm{B}}\right), 1.45(\mathrm{~s}, 9 \mathrm{H}, 12-\mathrm{H}), 1.44-1.36(\mathrm{~m}, 1 \mathrm{H}$, $\left.4-\mathrm{H}_{B}\right)$.

${ }^{13}$ C-NMR (101 MHz, tetrachlorethane- $\left.d_{2}, 373 \mathrm{~K}\right): \delta(\mathrm{ppm})=171.7(C=\mathrm{O}, \mathrm{C} 6), 170.3(C=\mathrm{O}, \mathrm{C} 7), 155.0(C=\mathrm{O}, \mathrm{C} 10)$, $80.5\left(\mathrm{C}\left(\mathrm{CH}_{3}\right)_{3}, \mathrm{C} 11\right), 55.9(\mathrm{CH}, \mathrm{C} 1), 51.9\left(\mathrm{CH}_{3}, \mathrm{C} 9\right), 51.7\left(\mathrm{CH}_{3}, \mathrm{C} 8\right), 43.5(\mathrm{CH}, \mathrm{C} 2), 41.3\left(\mathrm{CH}_{2}, \mathrm{C} 5\right), 28.5\left(\mathrm{C}(\mathrm{CH})_{3}\right.$, C12), $24.3\left(\mathrm{CH}_{2}, \mathrm{C} 4\right), 22.6\left(\mathrm{CH}_{2}, \mathrm{C} 3\right)$. 


\section{SS66T-type precursor}

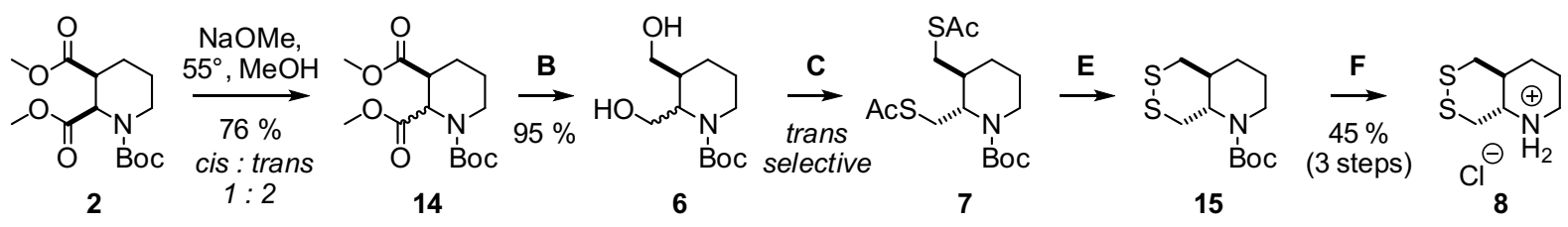

trans-hexahydro-[1,2]dithiino[4,5-b]pyridine hydrochloride (8)

Step 1: A mixture of 2 (5.33 g, $17.7 \mathrm{mmol}, 1.0$ eq.), anhydrous $\mathrm{MeOH}(50 \mathrm{~mL})$ and $\mathrm{NaOMe}(1.15 \mathrm{~g}, 21.2 \mathrm{mmol}$, 1.2 eq.) was stirred under nitrogen atmosphere at $55^{\circ} \mathrm{C}$ for $15 \mathrm{~h}$, before $\mathrm{AcOH}$ (1.52 mL, $26.5 \mathrm{mmol}, 1.5$ eq.) was added and all volatiles were removed under reduced pressure. The residue was suspended in DCM and filtered. The solids were rinsed with a small amount of DCM and discarded. The filtrate was diluted with hexane and directly purified by FCC (isohexane:EtOAc, 4:1) to afford a cis/trans mixture of $N$-Boc dimethyl piperidine-2,3dicarboxylate $14(4.08 \mathrm{~g}, 13.5 \mathrm{mmol}, 76 \% ; 62 \%$ trans) as a yellowish oil. The cis/trans ratio was determined to be 0.6 (cis): 1.0 (trans) by ${ }^{1} \mathrm{H}-\mathrm{NMR}$ spectroscopy $\left(\mathrm{CDCl}_{3}\right)$ at $298 \mathrm{~K}$ based on the intensity of the signals at 5.50 and $5.26 \mathrm{ppm}$ (cis) and the signal at $5.40 \mathrm{ppm}$ (trans).

Step 2: To a suspension of $\mathrm{LiAlH}_{4}(1.54 \mathrm{~g}, 40.6 \mathrm{mmol}, 3.0$ eq. $)$ in anhydrous THF $(80 \mathrm{~mL})$ at $0{ }^{\circ} \mathrm{C}$ under nitrogen atmosphere was dropwise added a solution of the material obtained in step 1 (4.08 g, $13.5 \mathrm{mmol}, 1.0 \mathrm{eq}$.) in anhydrous THF $(5.5 \mathrm{~mL})$. The mixture was stirred for $30 \mathrm{~min}$, the cooling bath was removed and stirring continued for $2 \mathrm{~h}$ at r.t. . The mixture as again cooled to $0^{\circ} \mathrm{C}$, then EtOAc $(5 \mathrm{~mL})$, MeOH $(5 \mathrm{~mL})$, water $(10 \mathrm{~mL})$ and $2 \mathrm{M} \mathrm{aq}$. $\mathrm{NaOH}(10 \mathrm{~mL})$ were added carefully in the specified order. The cooling bath was removed and the mixture was stirred at r.t. for $1 \mathrm{~h}$, before being filtered. The solids were washed with a small amount of THF and discarded. The filtrate was evaporated under reduced pressure, $\mathrm{Et}_{2} \mathrm{O}(50 \mathrm{~mL})$ and $\mathrm{Na}_{2} \mathrm{SO}_{4}(30 \mathrm{~g})$ were added and the mixture was filtered. The solids were washed with $\mathrm{Et}_{2} \mathrm{O}$ and discarded. The filtrate was evaporated under reduced pressure and remaining yellowish oil containing $6(3.16 \mathrm{~g}, 12.9 \mathrm{mmol}, 95 \%)$ was used in the next step without further purification.

Step 3: The material obtained in step $2(2.35 \mathrm{~g}, 9.58 \mathrm{mmol})$ was treated according to general protocol C. The diol solution and the HSAc were premixed and added to the reaction mixture simultaneously instead of successively. Instead of purification by FCC the residue obtained after removal of the volatiles was suspended in a mixture of $\mathrm{Et}_{2} \mathrm{O} /$ isohexane $(1: 2,150 \mathrm{~mL})$ and filtered. The solids were washed with additional $\mathrm{Et}_{2} \mathrm{O} /$ hexane $(1 / 2,60 \mathrm{~mL})$ and discarded. The filtrate was evaporated under reduced pressure, isohexane $(50 \mathrm{~mL})$ was added and the mixture was filtered again. The solids were washed with a small amount of isohexane and discarded. The filtrate was evaporated under reduced pressure and the remaining yellow oil containing $7(4.93 \mathrm{~g})$ was used in the next step without purification.

Step 4: The material obtained in step 3 was dissolved in $\mathrm{MeOH}(200 \mathrm{~mL})$ and $\mathrm{KOH}(1.68 \mathrm{~g}, 29.9 \mathrm{mmol})$ was added. The mixture was stirred open to air for $15 \mathrm{~h}$, before AcOH $(1.72 \mathrm{~mL}, 30.1 \mathrm{mmol})$ was added and all volatiles were removed under reduced pressure. The residue was suspended in EtOAc $(40 \mathrm{~mL})$ and filtered. The solids were washed with additional EtOAc $(2 \times 20 \mathrm{~mL})$ and discarded. The filtrate was evaporated under reduced pressure and the residue was partially purified by FCC (isohexane:EtOAc, 10:1) to remove tetrahydrofuran $\mathbf{5}$, which afforded a yellowish oil $(1.50 \mathrm{~g})$ containing 15<smiles>C1CCC2N[Hg]CCC2CSSC1</smiles>

Step 5: To the impure 15 obtained in step 4 was added a $4 \mathrm{M}$ solution of $\mathrm{HCl}$ in dioxane $(6.0 \mathrm{~mL}, 24 \mathrm{mmol})$. The mixture was stirred for $2 \mathrm{~h}$, before $\mathrm{Et}_{2} \mathrm{O}(50 \mathrm{~mL})$ was added and stirring was continued for several minutes. The precipitate was collected, washed with additional $\mathrm{Et}_{2} \mathrm{O}$ and dried to afford 8 ( $908 \mathrm{mg}, 4.29 \mathrm{mmol}, 45 \%$ over 3 steps) as a white solid.

${ }^{1} \mathrm{H}-\mathrm{NMR}\left(400 \mathrm{MHz}, \mathrm{DMSO}-\mathrm{d}_{6}\right): 9.34(\mathrm{~d}, \mathrm{~J}=20.9 \mathrm{~Hz}, 2 \mathrm{H}, 8-\mathrm{H}), 3.29-3.16\left(\mathrm{~m}, 2 \mathrm{H}, 1-\mathrm{H} ; 5-\mathrm{H}_{\mathrm{A}}\right), 3.11$ (dd, $\mathrm{J}=13.0$, $\left.3.1 \mathrm{~Hz}, 1 \mathrm{H}, 7-\mathrm{H}_{\mathrm{A}}\right), 3.00\left(\mathrm{dd}, J=12.9,11.3 \mathrm{~Hz}, 1 \mathrm{H}, 7-\mathrm{H}_{\mathrm{B}}\right), 2.97-2.84\left(\mathrm{~m}, 2 \mathrm{H}, 5-\mathrm{H}_{\mathrm{B}} ; 6-\mathrm{H}_{\mathrm{A}}\right), 2.82(\mathrm{dd}, J=13.8$, $\left.10.9 \mathrm{~Hz}, 1 \mathrm{H}, 6-\mathrm{H}_{\mathrm{B}}\right), 2.01$ (tdd, $\left.J=10.9,7.4,3.3 \mathrm{~Hz}, 1 \mathrm{H}, 2-\mathrm{H}\right), 1.82-1.69(\mathrm{~m}, 2 \mathrm{H}, 4-\mathrm{H}), 1.64(\mathrm{~d}, J=13.1 \mathrm{~Hz}, 1 \mathrm{H}$, $\left.3-\mathrm{H}_{\mathrm{A}}\right), 1.34\left(\mathrm{dt}, J=21.0,12.4 \mathrm{~Hz}, 1 \mathrm{H}, 3-\mathrm{H}_{\mathrm{A}}\right)$.

${ }^{13} \mathrm{C}-\mathrm{NMR}$ (101 MHz, DMSO-d $\left.\mathrm{d}_{6}\right): 59.4(\mathrm{CH}, \mathrm{C} 1), 43.9\left(\mathrm{CH}_{2}, \mathrm{C} 5\right), 40.2(\mathrm{CH}, \mathrm{C} 2) 38.6\left(\mathrm{CH}_{2}, \mathrm{C} 6\right), 34.2\left(\mathrm{CH}_{2}, \mathrm{C} 7\right), 28.8$ $\left(\mathrm{CH}_{2}, \mathrm{C} 3\right), 21.9\left(\mathrm{CH}_{2}, \mathrm{C} 4\right)$.

During the experiments that were performed while establishing the synthetic sequence described above, pure samples of $\mathbf{7}$ and $\mathbf{1 5}$ have been obtained. The analytical data for these compounds is reported in the following. 
trans-N-Boc 2,3-bis((acetylthio)methyl)piperidine (7)<smiles>CC(=O)SCC1CCCN(C(=O)OC(C)(C)C)[C@H]1CSC(C)=O</smiles>

TLC $R_{f}=0.50$ (isohexane:EtOAc, 3:1). $\mathrm{HRMS}\left(\mathrm{ESI}^{+}\right): \mathrm{C}_{16} \mathrm{H}_{27} \mathrm{NNaO}_{4} \mathrm{~S}_{2}{ }^{+}[\mathrm{M}+\mathrm{Na}]^{+}$: calc. 384.12737, found 384.12765.

${ }^{1} \mathrm{H}-\mathrm{NMR}\left(400 \mathrm{MHz}, \mathrm{CDCl}_{3}\right): \delta(\mathrm{ppm})=4.26(\mathrm{~s}, 1 \mathrm{H}, 1-\mathrm{H}), 4.15-3.80\left(\mathrm{~m}, 1 \mathrm{H}, 7-\mathrm{H}_{\mathrm{A}}\right), 3.33-3.13\left(\mathrm{~m}, 1 \mathrm{H}, 6-\mathrm{H}_{\mathrm{A}}\right), 3.06$ $\left(\mathrm{s}, 1 \mathrm{H}, 6-\mathrm{H}_{\mathrm{B}}\right), 2.99\left(\mathrm{dd}, J=13.7,6.4 \mathrm{~Hz}, 1 \mathrm{H}, 5-\mathrm{H}_{\mathrm{A}}\right), 2.88\left(\mathrm{dd}, J=13.7,8.4 \mathrm{~Hz}, 1 \mathrm{H}, 5-\mathrm{H}_{\mathrm{B}}\right), 2.73\left(\mathrm{~s}, 1 \mathrm{H}, 7-\mathrm{H}_{\mathrm{B}}\right), 2.33$ $(\mathrm{s}, 3 \mathrm{H}, 10-\mathrm{H}), 2.32(\mathrm{~s}, 3 \mathrm{H}, 11-\mathrm{H}), 1.89-1.74\left(\mathrm{~m}, 2 \mathrm{H}, 2-\mathrm{H} ; 3-\mathrm{H}_{\mathrm{A}}\right), 1.70-1.52\left(\mathrm{~m}, 2 \mathrm{H}, 3-\mathrm{H}_{\mathrm{B}} ; 4-\mathrm{H}_{\mathrm{A}}\right), 1.45(\mathrm{~s}, 9 \mathrm{H}$, $14-\mathrm{H}), 1.44-1.39\left(\mathrm{~m}, 1 \mathrm{H}, 4-\mathrm{H}_{\mathrm{B}}\right)$.

${ }^{13}$ C-NMR (101 MHz, $\left.\mathrm{CDCl}_{3}\right): \delta(\mathrm{ppm})=195.7(\mathrm{C}=\mathrm{O}, \mathrm{C} 8), 195.3(\mathrm{C}=\mathrm{O}, \mathrm{C} 9), 155.4(\mathrm{C}=\mathrm{O}, \mathrm{C} 12), 80.0\left(\left(\mathrm{C}\left(\mathrm{CH}_{3}\right)_{3}\right.\right.$, C13), 52.6 ( $\mathrm{CH}, \mathrm{C} 1), 37.7\left(\mathrm{CH}_{2}, \mathrm{C} 7\right), 36.3(\mathrm{CH}, \mathrm{C} 2), 31.4\left(\mathrm{CH}_{2}, \mathrm{C} 5\right), 30.8\left(\mathrm{CH}_{3}, \mathrm{C} 10\right), 30.7\left(\mathrm{CH}_{3}, \mathrm{C} 11\right), 29.8\left(\mathrm{CH}_{2}\right.$, C6), $28.5\left(\mathrm{CH}_{3}, \mathrm{C} 14\right), 23.1\left(\mathrm{CH}_{2}, \mathrm{C} 3\right), 19.9\left(\mathrm{CH}_{2}, \mathrm{C} 4\right)$.

trans-N-Boc hexahydro-[1,2]dithiino[4,5-b]pyridine (15)<smiles>CC(C)(C)OC(=O)N1CCCC2CSCC21</smiles>

TLC $R_{f}=0.72$ (isohexane:EtOAc, 4:1). HRMS $\left(\mathrm{ESI}^{+}\right.$): $\mathrm{C}_{12} \mathrm{H}_{22} \mathrm{NNaO}_{2} \mathrm{~S}_{2}{ }^{+}\left[\mathrm{M}^{-} \mathrm{C}_{4} \mathrm{H}_{8}\right]^{+}$: calc. 220.04605, found 220.04617.

${ }^{1} \mathrm{H}-\mathrm{NMR}\left(500 \mathrm{MHz}, \mathrm{CDCl}_{3}\right): \delta(\mathrm{ppm})=3.75$ (ddd, $J=14.0,7.4,2.0 \mathrm{~Hz}, 1 \mathrm{H}, 5-\mathrm{H}_{\mathrm{A}}$ ), 3.64 (ddd, $J=10.9,7.7,5.6 \mathrm{~Hz}$, $1 \mathrm{H}, 1-\mathrm{H}), 3.06$ (ddd, $\left.J=14.0,11.2,5.8 \mathrm{~Hz}, 1 \mathrm{H}, 5-\mathrm{H}_{\mathrm{B}}\right), 2.98\left(\mathrm{~d}, J=2.1 \mathrm{~Hz}, 1 \mathrm{H}, 7-\mathrm{H}_{\mathrm{A}}\right), 2.97\left(\mathrm{~s}, 1 \mathrm{H}, 7-\mathrm{H}_{\mathrm{B}}\right), 2.88$ (dd, $\left.J=13.5,10.8 \mathrm{~Hz}, 1 \mathrm{H}, 6-\mathrm{H}_{\mathrm{A}}\right), 2.75\left(\mathrm{dd}, J=13.5,2.8 \mathrm{~Hz}, 1 \mathrm{H}, 6-\mathrm{H}_{\mathrm{B}}\right), 2.03(\mathrm{dtt}, J=13.7,10.8,2.9 \mathrm{~Hz}, 1 \mathrm{H}, 2-\mathrm{H}), 1.84$ (ddddd, $\left.J=13.0,11.3,9.3,7.4,1.7 \mathrm{~Hz}, 1 \mathrm{H}, 4-\mathrm{H}_{\mathrm{A}}\right), 1.70-1.63\left(\mathrm{~m}, 1 \mathrm{H}, 4-\mathrm{H}_{\mathrm{B}}\right), 1.56$ (dddd, $J=12.8,9.7,2.9,1.9 \mathrm{~Hz}$, $\left.1 \mathrm{H}, 3-\mathrm{H}_{\mathrm{A}}\right), 1.45(\mathrm{~s}, 9 \mathrm{H}, 10-\mathrm{H}), 1.35-1.19\left(\mathrm{~m}, 1 \mathrm{H}, 3-\mathrm{H}_{\mathrm{B}}\right)$.

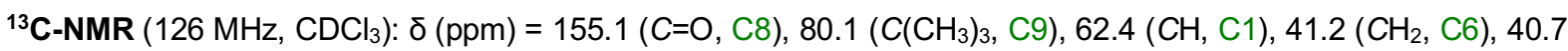
$(\mathrm{CH}, \mathrm{C} 2), 38.2\left(\mathrm{CH}_{2}, \mathrm{C} 5\right), 37.3\left(\mathrm{CH}_{2}, \mathrm{C} 7\right), 28.6\left(\mathrm{CH}_{3}, \mathrm{C} 10\right), 27.1\left(\mathrm{CH}_{2}, \mathrm{C} 3\right), 23.1\left(\mathrm{CH}_{2}, \mathrm{C} 4\right)$. 


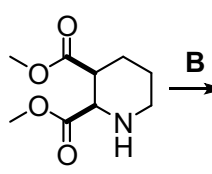

13<smiles>OCC1CCCNC1CO</smiles>

9

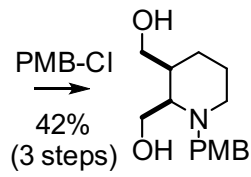

10<smiles>[R5][R15]([H])([H])N1CCCC(COC)C1CCl</smiles>

17

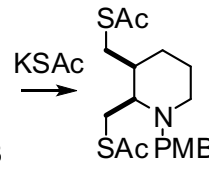

11

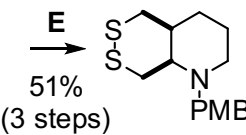

12

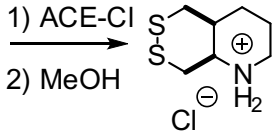

18

cis-N-(4-methoxybenzyl)-2,3-bis(hydroxymethyl)piperidine (10)<smiles>COc1ccc(CN2CCC[C@@H](CO)[C@H]2CO)cc1</smiles>

Step 1: To dimethyl pyridine-2,3-dicarboxylate $1(4.11 \mathrm{~g}, 21.1 \mathrm{mmol})$ in $\mathrm{MeOH}(30 \mathrm{~mL})$ was added $96 \% \mathrm{H}_{2} \mathrm{SO}_{4}$ $(0.11 \mathrm{~mL}, 2.1 \mathrm{mmol})$ and $10 \% \mathrm{Pd} / \mathrm{C}(125 \mathrm{mg}, 0.105 \mathrm{mmol})$. The mixture was stirred under hydrogen atmosphere (85 bar) for $15 \mathrm{~h}$, filtered over Celite ${ }^{\circledR}$ and concentrated to afford 13 as a colourless oil, which was used in the next step without purification.

Step 2: The material obtained in step 1 was mixed with anhydrous THF $(20 \mathrm{~mL})$, which dissolved most material. The solution was added dropwise to a suspension of $\mathrm{LiAlH}_{4}(2.40 \mathrm{~g}, 63.3 \mathrm{mmol}, 3.0 \mathrm{eq}$. $)$ in anhydrous THF $(125 \mathrm{~mL})$ at $0{ }^{\circ} \mathrm{C}$ under nitrogen atmosphere. Then, the remaining undissolved material from step 1 (a THFinsoluble, viscous mass) was added neat to the $\mathrm{LiAlH}_{4}$ suspension. The mixture was allowed to warm to r.t. and was further stirred at r.t. for $15 \mathrm{~h}$, was then cooled to $0{ }^{\circ} \mathrm{C}$ and EtOAc $(5 \mathrm{~mL}), \mathrm{MeOH}(5 \mathrm{~mL})$, water $(10 \mathrm{~mL})$ and $2 \mathrm{M}$ aq. $\mathrm{NaOH}(10 \mathrm{~mL})$ were added carefully in the specified order. The cooling bath was removed and the mixture was stirred at r.t. for $1 \mathrm{~h}$, before being filtered. The solids were washed with THF $(2 \times 30 \mathrm{~mL})$ and discarded. The filtrate was evaporated under reduced pressure. The residue as taken up in THF (30 mL) and $\mathrm{MeOH}(10 \mathrm{~mL})$. After drying over $\mathrm{Na}_{2} \mathrm{SO}_{4}$ all volatiles were removed under reduced pressure and the residue containing $\mathbf{9}$ was directly used in the next step without further purification.

Step 3: To the material obtained in step 2 were added $\mathrm{EtOH}(45 \mathrm{~mL}), \mathrm{K}_{2} \mathrm{CO}_{3}(7.29 \mathrm{~g}, 52.8 \mathrm{mmol}, 2.5$ eq.) and PMB-Cl (4.29 mL, $31.7 \mathrm{mmol}, 1.5$ eq.). After the mixture had stirred at r.t. for $15 \mathrm{~h}$, the solids were filtered off, washed with $\mathrm{EtOH}$ and discarded. The filtrate was evaporated under reduced pressure, $2 \mathrm{M}$ aq. $\mathrm{HCl}(60 \mathrm{~mL}) \mathrm{was}$ added and the mixture was washed with $\mathrm{DCM}(3 \times 30 \mathrm{~mL})$. The organic washings were discarded, the aqueous solution was basified with $2 \mathrm{M}$ aq. $\mathrm{NaOH}(70 \mathrm{~mL})$ and extracted with DCM $(2 \times 60 \mathrm{~mL})$. The extracts were dried over $\mathrm{Na}_{2} \mathrm{SO}_{4}$ and evaporated under reduced pressure. The residue was purified by FCC (DCM:MeOH:conc. $\mathrm{NH}_{3}$, 95:5:0.5 to $90: 10: 1)$ to afford $10(2.36 \mathrm{~g}, 8.89 \mathrm{mmol}, 42 \%$ over 3 steps $)$ as a faint orange oil.

TLC $\mathrm{R}_{f}=0.20$ (EtOAc). HRMS $\left(\mathrm{ESI}^{+}\right): \mathrm{C}_{15} \mathrm{H}_{24} \mathrm{NO}_{3}{ }^{+}[\mathrm{M}+\mathrm{H}]^{+}$: calc. 266.17507, found 266.17468.

${ }^{1} \mathrm{H}-N M R\left(400 \mathrm{MHz}, \mathrm{CDCl}_{3}\right): \delta(\mathrm{ppm})=7.24(\mathrm{~d}, J=8.5 \mathrm{~Hz}, 2 \mathrm{H}, 10-\mathrm{H}), 6.86(\mathrm{~d}, J=8.6 \mathrm{~Hz}, 2 \mathrm{H}, 11-\mathrm{H}), 3.86(\mathrm{~d}$, $\left.J=13.1 \mathrm{~Hz}, 1 \mathrm{H}, 8-\mathrm{H}_{\mathrm{A}}\right), 3.81\left(\mathrm{t}, J=9.5 \mathrm{~Hz}, 1 \mathrm{H}, 6-\mathrm{H}_{\mathrm{A}}\right), 3.79(\mathrm{~s}, 3 \mathrm{H}, 13-\mathrm{H}), 3.71\left(\mathrm{~d}, J=13.1 \mathrm{~Hz}, 1 \mathrm{H}, 8-\mathrm{H}_{\mathrm{B}}\right), 3.67-$ $3.52\left(\mathrm{~m}, 3 \mathrm{H}, 6-\mathrm{H}_{\mathrm{B}} ; 7-\mathrm{H}\right), 2.98-2.90(\mathrm{~m}, 1 \mathrm{H}, 1-\mathrm{H}), 2.74\left(\mathrm{ddd}, J=13.6,10.6,3.3 \mathrm{~Hz}, 1 \mathrm{H}, 5-\mathrm{H}_{\mathrm{A}}\right), 2.49(\mathrm{dd}, J=13.5$, $\left.3.9 \mathrm{~Hz}, 1 \mathrm{H}, 5-\mathrm{H}_{\mathrm{B}}\right), 2.17(\mathrm{dd}, J=10.5,4.4 \mathrm{~Hz}, 1 \mathrm{H}, 2-\mathrm{H}), 1.74-1.60(\mathrm{~m}, 2 \mathrm{H}, 3-\mathrm{H}), 1.54-1.37(\mathrm{~m}, 2 \mathrm{H}, 4-\mathrm{H})$.

${ }^{13} \mathrm{C}$-NMR $(101 \mathrm{MHz}, \mathrm{CDCl}): \delta(\mathrm{ppm})=159.0\left(C_{\mathrm{Ar}}, \mathrm{C} 9\right), 130.8\left(C_{\mathrm{Ar}}, \mathrm{C} 12\right), 130.1\left(C_{\mathrm{Ar}} \mathrm{H}, \mathrm{C} 10\right), 114.0\left(C_{\mathrm{Ar}} \mathrm{H}, \mathrm{C} 11\right)$, $64.9\left(\mathrm{CH}_{2}, \mathrm{C} 8\right), 60.8(\mathrm{CH}, \mathrm{C} 1), 57.5\left(\mathrm{CH}_{2}, \mathrm{C} 6\right), 57.3\left(\mathrm{CH}_{2}, \mathrm{C} 7\right), 55.4\left(\mathrm{CH}_{2}, \mathrm{C} 13\right), 46.4\left(\mathrm{CH}_{2}, \mathrm{C} 5\right), 36.4(\mathrm{CH}, \mathrm{C} 2)$, $25.0\left(\mathrm{CH}_{2}, \mathrm{C} 3\right), 20.9\left(\mathrm{CH}_{2}, \mathrm{C} 4\right)$. 


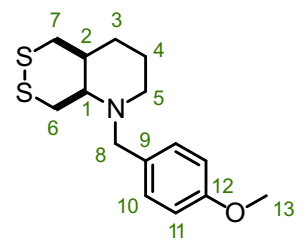

Step 1: To a solution of 10 (1.11 g, $4.18 \mathrm{mmol}, 1.0$ eq.) and $\mathrm{Et}_{3} \mathrm{~N}(2.92 \mathrm{~mL}, 20.9 \mathrm{mmol}, 5.0$ eq.) in anhydrous DCM $(45 \mathrm{~mL})$ at $0{ }^{\circ} \mathrm{C}$ under nitrogen atmosphere, was dropwise added $\mathrm{MsCl}(0.664 \mathrm{~mL}, 8.58 \mathrm{mmol}, 2.05$ eq. $)$. The mixture was stirred for $1 \mathrm{~h}$, then the cooling bath was removed. After the mixture had stirred at r.t. for $1 \mathrm{~h}$, it was washed with water $(50 \mathrm{~mL})$ and the aqueous washings were extracted with $\mathrm{DCM}(2 \times 20 \mathrm{~mL})$. The combined organic layers were dried over $\mathrm{Na}_{2} \mathrm{SO}_{4}$, evaporated under reduced pressure and the residue containing 17 was directly used in the next step without purification.

Step 2: The material obtained in step 1 was dissolved in anhydrous DMF (85 mL), KSAc (1.43 g, $12.5 \mathrm{mmol}$, 3.0 eq.) was added and the mixture was stirred at r.t. for $15 \mathrm{~h}$. Then, the mixture was evaporated under reduced pressure and the residue was suspended in $\mathrm{Et}_{2} \mathrm{O}(50 \mathrm{~mL})$. The solids were filtered off, washed with additional $\mathrm{Et}_{2} \mathrm{O}$ $(4 \times 10 \mathrm{~mL})$ and discarded. The filtrate was evaporated under reduced pressure and the residue was partially purified by FCC (isohexane:EtOAc, 4:1), which afforded an orange oil (922 mg) containing 11 that was used in the next step without further purification.

Step 3: The material obtained in step 2 was dissolved in $\mathrm{MeOH}(50 \mathrm{~mL}), \mathrm{KOH}(420 \mathrm{mg})$ was added and the mixture was stirred open to air for $72 \mathrm{~h}$. Then, $\mathrm{AcOH}(0.5 \mathrm{~mL})$ was added, all volatiles were removed under reduced pressure and the residue was suspended in EtOAc $(20 \mathrm{~mL})$. The solids were filtered off, washed with additional EtOAc $(2 \times 10 \mathrm{~mL})$ and discarded. The filtrate was evaporated under reduced pressure and the residue was purified by FCC ((isohexane:EtOAc, 6:1) to afford 12 (629 mg, $2.13 \mathrm{mmol}, 51 \%$ over 3 steps) as a yellowish oil.

TLC $\mathrm{R}_{f}=0.47$ (isohexane:EtOAc, 4:1). HRMS (ESI ${ }^{+}$): $\mathrm{C}_{15} \mathrm{H}_{22} \mathrm{NOS}_{2}{ }^{+}[\mathrm{M}+\mathrm{H}]^{+}$: calc. 296.11373, found 296.11341.

${ }^{1} \mathrm{H}-N M R\left(400 \mathrm{MHz}, \mathrm{DMSO}_{-} \mathrm{d}_{6}\right): \delta(\mathrm{ppm})=7.22(\mathrm{~d}, J=8.2 \mathrm{~Hz}, 2 \mathrm{H}, 10-\mathrm{H}), 6.87(\mathrm{~d}, J=8.5 \mathrm{~Hz}, 2 \mathrm{H}, 11-\mathrm{H}), 3.73(\mathrm{~s}$, $3 \mathrm{H}, 13-\mathrm{H}), 3.66\left(\mathrm{~d}, J=12.5 \mathrm{~Hz}, 1 \mathrm{H}, 8-\mathrm{H}_{\mathrm{A}}\right), 3.52\left(\mathrm{~d}, J=12.8 \mathrm{~Hz}, 1 \mathrm{H}, 8-\mathrm{H}_{\mathrm{B}}\right), 3.40-3.31\left(\mathrm{~m}, 1 \mathrm{H}, 6-\mathrm{H}_{\mathrm{A}}\right), 3.25(\mathrm{~d}$, $\left.J=13.0 \mathrm{~Hz}, 1 \mathrm{H}, 5-\mathrm{H}_{\mathrm{A}}\right), 2.94(\mathrm{~d}, J=9.6 \mathrm{~Hz}, 1 \mathrm{H}, 1-\mathrm{H}), 2.84\left(\mathrm{~d}, J=12.9 \mathrm{~Hz}, 1 \mathrm{H}, 5-\mathrm{H}_{\mathrm{B}}\right), 2.70(\mathrm{dd}, J=12.3,7.1 \mathrm{~Hz}$, $\left.1 \mathrm{H}, 6-\mathrm{H}_{\mathrm{B}}\right), 2.49-2.32(\mathrm{~m}, 2 \mathrm{H}, 7-\mathrm{H}), 2.25-2.14(\mathrm{~m}, 1 \mathrm{H}, 2-\mathrm{H}), 2.09\left(\mathrm{~d}, J=10.9 \mathrm{~Hz}, 1 \mathrm{H}, 4-\mathrm{H}_{\mathrm{A}}\right), 1.69-1.56(\mathrm{~m}, 1 \mathrm{H}$, $\left.3-\mathrm{H}_{\mathrm{A}}\right), 1.56-1.48\left(\mathrm{~m}, 1 \mathrm{H}, 3-\mathrm{H}_{\mathrm{B}}\right), 1.34\left(\mathrm{~d}, J=11.8 \mathrm{~Hz}, 1 \mathrm{H}, 4-\mathrm{H}_{\mathrm{B}}\right) .{ }^{13} \mathrm{C}-\mathrm{NMR}\left(101 \mathrm{MHz}, \mathrm{DMSO}-\mathrm{d}_{6}\right): \delta(\mathrm{ppm})=158.2$ $\left(C_{\mathrm{Ar}}, \mathrm{C} 9\right), 131.1\left(\mathrm{C}_{\mathrm{Ar}}, \mathrm{C} 12\right), 129.5\left(\mathrm{C}_{\mathrm{Ar}} \mathrm{H}, \mathrm{C} 10\right), 113.6\left(\mathrm{C}_{\mathrm{Ar}} \mathrm{H}, \mathrm{C} 11\right), 58.2(\mathrm{CH}, \mathrm{C} 1), 56.5\left(\mathrm{CH}_{2}, \mathrm{C} 8\right), 55.0\left(\mathrm{CH}_{3}, \mathrm{C} 13\right)$, $45.0\left(\mathrm{CH}_{2}, \mathrm{C} 7\right), 41.8\left(\mathrm{CH}_{2}, \mathrm{C} 5\right), 36.8(\mathrm{CH}, \mathrm{C} 2), 25.0\left(\mathrm{CH}_{2}, \mathrm{C} 3 ; 6-\mathrm{H}\right), 22.6\left(\mathrm{CH}_{2}, \mathrm{C} 4\right)$.

During the experiments that were performed while establishing the synthetic sequence described above, a pure sample of $\mathbf{1 1}$ has been obtained and the analytical data for this compound is reported in the following.

cis-N-((1-(4-methoxybenzyl)piperidine-2,3-diyl)bis(methylene)) diethanethioate (11)<smiles>COc1ccc(CN2CCCC(COC(C)=O)C2CSC(C)=O)cc1</smiles>

TLC $\mathrm{R}_{f}=0.36$ (isohexane:EtOAc, 4:1). $\mathrm{HRMS}\left(\mathrm{ESI}^{+}\right): \mathrm{C}_{19} \mathrm{H}_{28} \mathrm{NO}_{3} \mathrm{~S}_{2}{ }^{+}[\mathrm{M}+\mathrm{H}]^{+}$: calc. 382.15051, found 382.15002.

${ }^{1} \mathrm{H}-\mathrm{NMR}\left(400 \mathrm{MHz}, \mathrm{CDCl}_{3}\right): \delta(\mathrm{ppm})=7.24(\mathrm{~d}, J=8.4 \mathrm{~Hz}, 2 \mathrm{H}, 14-\mathrm{H}), 6.84(\mathrm{~d}, J=8.6 \mathrm{~Hz}, 2 \mathrm{H}, 15-\mathrm{H}), 3.80(\mathrm{~s}, 3 \mathrm{H}$, $17-\mathrm{H}), 3.67(\mathrm{~s}, 2 \mathrm{H}, 12-\mathrm{H}), 3.25$ (dd, $\left.J=13.6,8.0 \mathrm{~Hz}, 1 \mathrm{H}, 6-\mathrm{H}_{\mathrm{A}}\right), 2.99\left(\mathrm{dd}, J=13.6,5.4 \mathrm{~Hz}, 1 \mathrm{H}, 6-\mathrm{H}_{\mathrm{B}}\right), 2.91$ (dt, $J=8.5,4.5 \mathrm{~Hz}, 1 \mathrm{H}, 1-\mathrm{H}), 2.88-2.77\left(\mathrm{~m}, 2 \mathrm{H}, 7-\mathrm{H}_{\mathrm{A}}\right), 2.60\left(\mathrm{td}, J=12.3,11.3,3.2 \mathrm{~Hz}, 1 \mathrm{H}, 5-\mathrm{H}_{\mathrm{A}}\right), 2.44(\mathrm{~d}, J=13.3 \mathrm{~Hz}$, $\left.1 \mathrm{H}, 5-\mathrm{H}_{\mathrm{B}}\right), 2.33(\mathrm{~s}, 3 \mathrm{H}, 10-\mathrm{H}), 2.33(\mathrm{~s}, 3 \mathrm{H}, 11-\mathrm{H}), 2.13-2.00(\mathrm{~m}, 1 \mathrm{H}, 2-\mathrm{H}), 1.75-1.65\left(\mathrm{~m}, 1 \mathrm{H}, 3-\mathrm{H}_{\mathrm{A}}\right), 1.63-1.53$ $\left(\mathrm{m}, 1 \mathrm{H}, 4-\mathrm{H}_{\mathrm{A}}\right), 1.48-1.40\left(\mathrm{~m}, 1 \mathrm{H}, 4-\mathrm{H}_{\mathrm{B}}\right), 1.40-1.30\left(\mathrm{~m}, 1 \mathrm{H}, 3-\mathrm{H}_{\mathrm{B}}\right)$.

${ }^{13} \mathrm{C}-\mathrm{NMR}\left(101 \mathrm{MHz}, \mathrm{CDCl}_{3}\right): \delta(\mathrm{ppm})=196.4(C=\mathrm{O}, \mathrm{C} 8), 195.8(\mathrm{C}=\mathrm{O}, \mathrm{C9}), 158.7\left(C_{\mathrm{Ar}}, \mathrm{C} 13\right), 131.8\left(C_{\mathrm{Ar}}, \mathrm{C} 16\right)$, 129.8 ( $\left.\mathrm{C}_{\mathrm{Ar}} \mathrm{H}, \mathrm{C} 14\right), 113.7\left(\mathrm{C}_{\mathrm{Ar}} \mathrm{H}, \mathrm{C} 15\right), 61.0(\mathrm{CH}, \mathrm{C} 1), 57.8\left(\mathrm{CH}_{2}, \mathrm{C} 12\right), 55.4\left(\mathrm{CH}_{3}, \mathrm{C} 17\right), 45.3\left(\mathrm{CH}_{2}, \mathrm{C} 5\right), 37.6(\mathrm{CH}$, $\mathrm{C} 2), 32.0\left(\mathrm{CH}_{2}, \mathrm{C} 7\right), 30.8\left(\mathrm{CH}_{3}, \mathrm{C} 10\right), 30.7\left(\mathrm{CH}_{3}, \mathrm{C} 11\right), 26.0\left(\mathrm{CH}_{2}, \mathrm{C} 6\right), 25.5\left(\mathrm{CH}_{2}, \mathrm{C} 3\right), 22.3\left(\mathrm{CH}_{2}, \mathrm{C} 4\right)$. 
<smiles>C1CCC2NNCCC2CSSC1</smiles>

To a solution of 12 (494 mg, $1.67 \mathrm{mmol}, 1.0$ eq.) in anhydrous THF (5 mL) at $0^{\circ} \mathrm{C}$ was dropwise added 1-chloroethyl chloroformate (ACE-Cl) $(362 \mu \mathrm{L}, 3.34 \mathrm{mmol}, 2.0 \mathrm{eq}$.). The mixture was warmed to r.t. and stirred for three days, before $\mathrm{Et}_{2} \mathrm{O}(30 \mathrm{~mL})$ was added and the mixture was washed with $2 \mathrm{M}$ aq. $\mathrm{HCl}(25 \mathrm{~mL})$. The aq. washings were extracted with $\mathrm{Et}_{2} \mathrm{O}(2 \times 30 \mathrm{~mL})$, basified by addition of $2 \mathrm{M}$ aq. $\mathrm{NaOH}(30 \mathrm{~mL})$ and unreacted $12(89 \mathrm{mg}, 0.30 \mathrm{mmol}$, $18 \%$ ) was recovered after extracting the basified extracts with $\mathrm{Et}_{2} \mathrm{O}$, drying over $\mathrm{MgSO}_{4}$ and removal of the solvent under reduced pressure. The extracts that were obtained before basifying the aq. washings were dried over $\mathrm{MgSO}_{4}$ and evaporated under reduced pressure. The residue was purified by FCC (DCM/isohexane), collecting a fraction with $\mathrm{R}_{\mathrm{f}}=0.5(\mathrm{DCM})$. To the resulting colourless oil was added anhydrous $\mathrm{MeOH}(50 \mathrm{~mL})$, the mixture was stirred at reflux for $1 \mathrm{~h}$ and evaporated under reduced pressure. The residue was washed with $\mathrm{Et}_{2} \mathrm{O}$ to obtain 18 (207 mg, $0.977 \mathrm{mmol}, 58 \%$ ) as an off-white solid.

Broad signals were observed by NMR spectroscopy at $298 \mathrm{~K}$, which was attributed to a slow interconversion between conformers, and sharp signals were observed by measurements at $373 \mathrm{~K}$.

${ }^{1} \mathrm{H}-\mathrm{NMR}\left(400 \mathrm{MHz}, \mathrm{DMSO}-\mathrm{d}_{6}, 373 \mathrm{~K}\right): \delta(\mathrm{ppm})=9.55(\mathrm{~s}, 2 \mathrm{H}), 3.66(\mathrm{dt}, \mathrm{J}=6.7,3.2 \mathrm{~Hz}, 1 \mathrm{H}), 3.51(\mathrm{dd}, \mathrm{J}=13.9$, $7.6 \mathrm{~Hz}, 1 \mathrm{H}), 3.29(\mathrm{~s}, 1 \mathrm{H}), 3.14(\mathrm{~d}, \mathrm{~J}=12.9 \mathrm{~Hz}, 2 \mathrm{H}), 3.04-2.85(\mathrm{~m}, 1 \mathrm{H}), 2.35(\mathrm{~s}, 1 \mathrm{H}), 1.91(\mathrm{~s}, 1 \mathrm{H}), 1.82$ (ddq, $J=12.6,8.4,4.3 \mathrm{~Hz}, 1 \mathrm{H}), 1.76-1.65(\mathrm{~m}, 1 \mathrm{H}), 1.61(\mathrm{td}, J=9.2,4.5 \mathrm{~Hz}, 1 \mathrm{H})$.

\section{O56-type precursor and side products}<smiles></smiles>

2

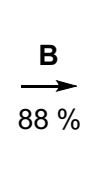

$\overrightarrow{88 \%}$

(1)<smiles>O=C(O)C1CCCN(C(=O)OCc2ccccc2)C1CO</smiles>

3

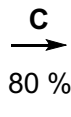

$80 \%$

cis-N-Boc 2,3-bis(hydroxymethyl)piperidine (3)<smiles>O=C(O)c1ccccc1</smiles>

5

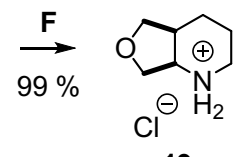

19<smiles>[2H]C(C)(C)OC(=O)N1CCC[C@@H](CO)[C@H]1CO</smiles>

Prepared from $2(10.7 \mathrm{~g}, 35.5 \mathrm{mmol})$ according to general protocol B. Compound $3(7.7 \mathrm{~g}, 31.4 \mathrm{mmol}, 88 \%)$ was obtained as a colourless oil and used without further purification.

TLC $\mathrm{R}_{f}=0.30$ (EtOAc). HRMS (El): $\mathrm{C}_{11} \mathrm{H}_{20} \mathrm{NO}_{3}{ }^{\cdot}\left[\mathrm{M}-\mathrm{CH}_{3} \mathrm{O}-\mathrm{e}\right]$ ': calc. 214.1438, found 214.1435. ${ }^{1} \mathrm{H}-\mathrm{NMR}(400 \mathrm{MHz}$, $\left.\mathrm{CDCl}_{3}\right): \delta(\mathrm{ppm})=4.41(\mathrm{~s}, 1 \mathrm{H}, 1-\mathrm{H}), 3.93\left(\mathrm{~s}, 1 \mathrm{H}, 5-\mathrm{H}_{\mathrm{A}}\right), 3.86\left(\mathrm{dd}, J=11.1,7.3 \mathrm{~Hz}, 1 \mathrm{H}, 7-\mathrm{H}_{\mathrm{A}}\right), 3.69-3.52(\mathrm{~m}, 3 \mathrm{H}$, $\left.6-\mathrm{H} ; 7-\mathrm{H}_{\mathrm{B}}\right), 3.08(\mathrm{~s}, 2 \mathrm{H}, 13-\mathrm{H} ; 14-\mathrm{H}), 2.73\left(\mathrm{~s}, 1 \mathrm{H}, 5-\mathrm{H}_{\mathrm{B}}\right), 1.93(\mathrm{dq}, J=7.9,3.7 \mathrm{~Hz}, 1 \mathrm{H}, 2-\mathrm{H}), 1.92-1.80(\mathrm{~m}, 1 \mathrm{H}$, 2-H), $1.69\left(\mathrm{~m}, 1 \mathrm{H}, 4-\mathrm{H}_{\mathrm{A}}\right), 1.63-1.52\left(\mathrm{~m}, 1 \mathrm{H}, 3-\mathrm{H}_{\mathrm{A}}\right), 1.45(\mathrm{~s}, 9 \mathrm{H}, 12-\mathrm{H}), 1.40\left(\mathrm{~m}, 2 \mathrm{H}, 3-\mathrm{H}_{\mathrm{B}} ;{ }^{4}-\mathrm{H}_{\mathrm{B}}\right) .{ }^{13} \mathrm{C}-\mathrm{NMR}$ $\left.\left(101 \mathrm{MHz}, \mathrm{CDCl}_{3}\right): \delta(\mathrm{ppm})=156.0(\mathrm{C}=\mathrm{O}, \mathrm{C} 10), 80.1\left(\mathrm{C}_{(\mathrm{CH}}\right)_{3}, \mathrm{C} 11\right), 68.1\left(\mathrm{CH}_{2}, \mathrm{C} 5\right), 64.6\left(\mathrm{CH}_{2}, \mathrm{C}\right), 59.6\left(\mathrm{CH}_{2}\right.$,

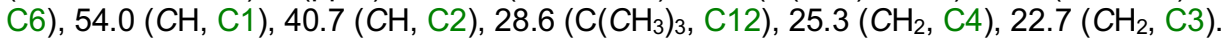

\section{cis-(3-oxohexahydro-3H-oxazolo[3,4-a]pyridin-8-yl)methyl methanesulfonate (4)}<smiles>CS(=O)(=O)OC[C@H]1CCCN2C(=O)OC[C@@H]12</smiles>

To a solution of 3 (372 mg, $1.51 \mathrm{mmol}, 1.0$ eq.) in anhydrous DCM (38 mL) at $0{ }^{\circ} \mathrm{C}$ under nitrogen atmosphere were added $\mathrm{Et}_{3} \mathrm{~N}$ (1.05 mL, $7.57 \mathrm{mmol}, 5.0$ eq.) and $\mathrm{MsCl}(0.29 \mathrm{~mL}, 3.79 \mathrm{mmol}, 2.5$ eq.). The mixture was stirred for $1 \mathrm{~h}$, then warmed to r.t. and stirred for further $12 \mathrm{~h}$, before washing with water $(50 \mathrm{~mL})$. The aqueous washings were extracted with $\mathrm{DCM}(2 \times 50 \mathrm{~mL})$ and the combined organic layers were dried over $\mathrm{Na}_{2} \mathrm{SO}_{4}$ and evaporated under reduced pressure. The residue was purified by FCC (isohexane/EtOAc) to afford 4 (336 mg, $1.35 \mathrm{mmol}$, $89 \%)$ as a colourless oil.

TLC $R_{f}=0.26$ (isohexane:EtOAc, 2:1). 
${ }^{1} \mathrm{H}-\mathrm{NMR}\left(400 \mathrm{MHz}, \mathrm{CDCl}_{3}\right): \delta(\mathrm{ppm})=4.42\left(\mathrm{dd}, J=10.6,7.1 \mathrm{~Hz}, 1 \mathrm{H}, 6-\mathrm{H}_{\mathrm{A}}\right), 4.35\left(\mathrm{t}, J=9.0 \mathrm{~Hz}, 1 \mathrm{H}, 7-\mathrm{H}_{\mathrm{A}}\right), 4.32-$ $4.25\left(\mathrm{~m}, 2 \mathrm{H}, 6-\mathrm{H}_{\mathrm{B}} ; 7-\mathrm{H}_{\mathrm{B}}\right), 3.94-3.87\left(\mathrm{~m}, 2 \mathrm{H}, 1-\mathrm{H} ; 5-\mathrm{H}_{\mathrm{A}}\right), 3.05(\mathrm{~s}, 3 \mathrm{H}, 9-\mathrm{H}), 2.90(\mathrm{ddd}, \mathrm{J}=13.1,10.9,5.4 \mathrm{~Hz}, 1 \mathrm{H}$, $\left.5-\mathrm{H}_{\mathrm{B}}\right), 2.30(\mathrm{tq}, \mathrm{J}=6.8,3.6 \mathrm{~Hz}, 1 \mathrm{H}, 2-\mathrm{H}), 2.14-2.02\left(\mathrm{~m}, 1 \mathrm{H}, 4-\mathrm{H}_{\mathrm{A}}\right), 1.76-1.48\left(\mathrm{~m}, 3 \mathrm{H}, 3-\mathrm{H} ; 4-\mathrm{H}_{\mathrm{B}}\right)$.

${ }^{13} \mathrm{C}-\mathrm{NMR}\left(101 \mathrm{MHz}, \mathrm{CDCl}_{3}\right): \delta(\mathrm{ppm})=157.3(\mathrm{C}=\mathrm{O}, \mathrm{C} 8), 67.3\left(\mathrm{CH}_{2}, \mathrm{C} 6\right), 64.5\left(\mathrm{CH}_{2}, \mathrm{C} 7\right), 56.4(\mathrm{CH}, \mathrm{C} 1), 41.6\left(\mathrm{CH}_{2}\right.$, C5), $37.6\left(\mathrm{CH}_{3}, \mathrm{C} 9\right), 34.9(\mathrm{CH}, \mathrm{C} 2), 26.0\left(\mathrm{CH}_{2}, \mathrm{C} 4\right), 19.1\left(\mathrm{CH}_{2}, \mathrm{C} 3\right)$.

\section{cis-N-Boc hexahydrofuro[3,4-b]pyridine (5)}<smiles>CC(C)(C)OC(=O)N1CCCC2COCC21</smiles>

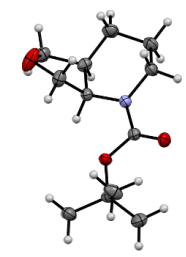

Prepared from $3(1.77 \mathrm{~g}, 7.14 \mathrm{mmol}$ ) according to general protocol C. Different concentrations than in the general protocol were employed: $0.11 \mathrm{M}$ for the $\mathrm{PPh}_{3}$ solution and $0.36 \mathrm{M}$ for the substrate solution. Before being concentrated, the reaction mixture was filtered to remove precipitated $\mathrm{S}=\mathrm{PPh}_{3}$ and $\mathrm{O}=\mathrm{PPh}_{3} . \mathbf{5}(1.3 \mathrm{~g}, 5.72 \mathrm{mmol}$, $80 \%$ ) was obtained as a colourless oil after FCC (isohexane/EtOAc). Crystals suitable for single crystal X-Ray crystallography were prepared by slow evaporation of a solution in DCM.

TLC $R_{f}=0.43$ (isohexane:EtOAc, 2:1). HRMS $\left(E S I^{+}\right): \mathrm{C}_{7} \mathrm{H}_{14} \mathrm{NO}^{+}[\mathrm{M}+\mathrm{H}]^{+}$: calc. 128.10699, found 128.10700. ${ }^{1} \mathrm{H}-N M R\left(400 \mathrm{MHz}, \mathrm{CDCl}_{3}\right): \delta(\mathrm{ppm})=4.65(\mathrm{~s}, 1 \mathrm{H}, 1-\mathrm{H}), 4.03-3.78\left(\mathrm{~m}, 3 \mathrm{H}, 3-\mathrm{H}_{\mathrm{A}} ; 4-\mathrm{H}_{\mathrm{A}} ; 5-\mathrm{H}_{\mathrm{A}}\right), 3.64(\mathrm{dd}, \mathrm{J}=8.7$, $\left.1.3 \mathrm{~Hz}, 1 \mathrm{H}, 5-\mathrm{H}_{\mathrm{B}}\right), 3.65\left(\mathrm{t}, J=8.6 \mathrm{~Hz}, 1 \mathrm{H}, 4-\mathrm{H}_{\mathrm{B}}\right), 2.74\left(\mathrm{t}, J=12.1 \mathrm{~Hz}, 1 \mathrm{H}, 3-\mathrm{H}_{\mathrm{B}}\right), 2.20-2.07(\mathrm{~m}, 1 \mathrm{H}, 2-\mathrm{H}), 1.80-$ $1.72\left(\mathrm{~m}, 1 \mathrm{H}, 7-\mathrm{H}_{\mathrm{A}}\right), 1.69\left(\mathrm{~s}, 1 \mathrm{H}, 6-\mathrm{H}_{\mathrm{A}}\right), 1.45(\mathrm{~s}, 9 \mathrm{H}, 10-\mathrm{H}), 1.43-1.39\left(\mathrm{~m}, 2 \mathrm{H}, 6-\mathrm{H}_{\mathrm{B}} ; 7-\mathrm{H}_{\mathrm{B}}\right) .{ }^{13} \mathrm{C}-\mathrm{NMR}(101 \mathrm{MHz}$,

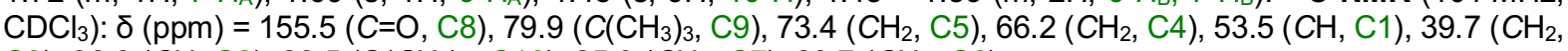
C3), $36.0(\mathrm{CH}, \mathrm{C} 2), 28.5\left(\mathrm{C}\left(\mathrm{CH}_{3}\right)_{3}, \mathrm{C} 10\right), 25.3\left(\mathrm{CH}_{2}, \mathrm{C} 7\right), 23.7\left(\mathrm{CH}_{2}, \mathrm{C} 6\right)$.

\section{cis-octahydrofuro[3,4-b]pyridine hydrochloride (19)}

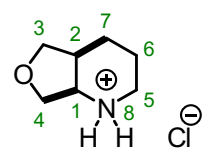

Prepared from 5 (145 mg, $0.64 \mathrm{mmol}$ ) according to general protocol $\mathbf{F}$ using a larger amount of $\mathrm{HCl}(15 \mathrm{eq}$.) to afford 19 (104 mg, $0.64 \mathrm{mmol}$, quant.) as a colourless crystalline solid.

${ }^{1} \mathrm{H}-N M R\left(500 \mathrm{MHz}, \mathrm{DMSO}-\mathrm{d}_{6}\right): \delta(\mathrm{ppm})=9.95\left(\mathrm{~s}, 1 \mathrm{H}, 8-\mathrm{H}_{\mathrm{A}}\right), 8.63\left(\mathrm{~s}, 1 \mathrm{H}, 8-\mathrm{H}_{\mathrm{B}}\right), 3.89-3.78\left(\mathrm{~m}, 3 \mathrm{H}, 3-\mathrm{H} ; 4-\mathrm{H}_{\mathrm{A}}\right)$, $3.71(\mathrm{q}, J=5.0,4.6 \mathrm{~Hz}, 1 \mathrm{H}, 1-\mathrm{H}), 3.66\left(\mathrm{dd}, J=9.7,8.3 \mathrm{~Hz}, 1 \mathrm{H}, 4-\mathrm{H}_{\mathrm{B}}\right), 3.07\left(\mathrm{~d}, J=11.9 \mathrm{~Hz}, 1 \mathrm{H}, 5-\mathrm{H}_{\mathrm{A}}\right), 2.83(\mathrm{~d}$, $\left.J=11.3 \mathrm{~Hz}, 1 \mathrm{H}, 5-\mathrm{H}_{\mathrm{A}}\right), 2.57-2.51(\mathrm{~m}, 1 \mathrm{H}, 2-\mathrm{H}), 1.83-1.72\left(\mathrm{~m}, 1 \mathrm{H}, 6-\mathrm{H}_{\mathrm{A}}\right), 1.64$ (dddd, $J=17.8,15.5,12.8,7.6 \mathrm{~Hz}$, $\left.3 \mathrm{H}, 6-\mathrm{H}_{\mathrm{B}} ; 7-\mathrm{H}\right) .{ }^{13} \mathrm{C}-\mathrm{NMR}\left(126 \mathrm{MHz}, \mathrm{DMSO}-\mathrm{d}_{6}\right): \delta(\mathrm{ppm})=69.9\left(\mathrm{CH}_{2}, \mathrm{C} 3\right), 68.1\left(\mathrm{CH}_{2}, \mathrm{C} 4\right), 55.3(\mathrm{CH}, \mathrm{C} 1), 41.5$ $\left(\mathrm{CH}_{2}, \mathrm{C} 5\right), 35.0(\mathrm{CH}, \mathrm{C} 2), 19.5\left(\mathrm{CH}_{2}, \mathrm{C} 6\right), 17.1\left(\mathrm{CH}_{2}, \mathrm{C} 7\right)$. 
SS60-type precursor: initial Mitsunobu route

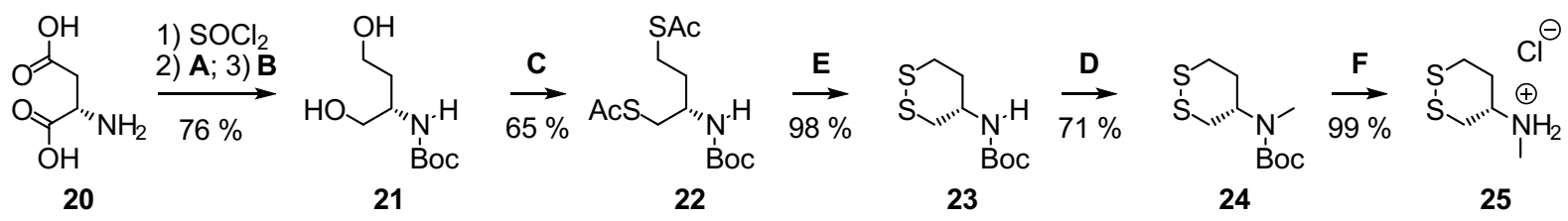

(S)-N-Boc-2-aminobutane-1,4-diol (21) $)^{22}$

Step 1: To a solution of $L$-aspartic acid $20(10.0 \mathrm{~g}, 75.1 \mathrm{mmol})$ in anhydrous $\mathrm{MeOH}(150 \mathrm{~mL})$ at $0{ }^{\circ} \mathrm{C}$ was dropwise added $\mathrm{SOCl}_{2}(16.2 \mathrm{~mL}, 223.3 \mathrm{mmol})$. The mixture was warmed to $50{ }^{\circ} \mathrm{C}$ and stirred at that temperature for $2 \mathrm{~h}$, before the volatiles were removed under reduced pressure. The residue was used in the next step without purification.

Step 2: The material obtained in step 1 was treated according to general protocol A. Different reaction conditions than in the general procedure were used: the reaction mixture was heated to $50{ }^{\circ} \mathrm{C}$ for $5 \mathrm{~h}$ instead of stirring at room temperature overnight. Dimethyl $N$-Boc- $L$-aspartate $(16.4 \mathrm{~g}, 62.8 \mathrm{mmol}, 84 \%$ over 2 steps) was obtained as a colourless solid after recrystallization from $\mathrm{MeOH}$.

Step 3: The material obtained in step $2(5.00 \mathrm{~g}, 19.1 \mathrm{mmol})$ was treated according to general protocol B. Compound 21 (3.55 g, $17.3 \mathrm{mmol}, 91 \%$ ) was obtained as a colourless solid after recrystallization from $\mathrm{Et}_{2} \mathrm{O}^{22}$

TLC $\mathrm{R}_{f}=0.29$ (isohexane:EtOAc, 1:1). HRMS $\left(\mathrm{ESI}^{+}\right): \mathrm{C}_{9} \mathrm{H}_{20} \mathrm{NO}_{4}{ }^{+}[\mathrm{M}+\mathrm{H}]^{+}$: calc. 206.1387, found 206.1384. ${ }^{1} \mathrm{H}-\mathrm{NMR}$ $\left(400 \mathrm{MHz}, \mathrm{CDCl}_{3}\right): \delta(\mathrm{ppm})=5.10(\mathrm{~d}, J=8.6 \mathrm{~Hz}, 1 \mathrm{H},-\mathrm{NH}), 3.83(\mathrm{q}, J=5.0 \mathrm{~Hz}, 1 \mathrm{H}), 3.71(\mathrm{~d}, J=11.9 \mathrm{~Hz}, 3 \mathrm{H}), 3.62$ (dd, $J=11.0,4.6 \mathrm{~Hz}, 1 \mathrm{H}), 3.51(\mathrm{~s}, 1 \mathrm{H}, \mathrm{OH}), 3.05(\mathrm{~s}, 1 \mathrm{H}, \mathrm{OH}), 1.80$ (ddt, $J=13.9,9.3,4.7 \mathrm{~Hz}, 1 \mathrm{H}), 1.61$ (tt, $J=9.0$, 7.3, $5.6 \mathrm{~Hz}, 1 \mathrm{H}), 1.44(\mathrm{~s}, 9 \mathrm{H})$.

\section{(S)-N-Boc-2-amino-1,4-bis(acetylthio)butane(22) ${ }^{22}$}

Prepared from $21(2.00 \mathrm{~g}, 9.7 \mathrm{mmol})$ according to general protocol C. Compound $22(2.01 \mathrm{~g}, 6.3 \mathrm{mmol}, 65 \%)$ was obtained as a colourless solid after $\mathrm{FCC}\left(n\right.$-pentane/Et $\left.\mathrm{E}_{2} \mathrm{O}\right){ }^{22}$

TLC $\mathrm{R}_{f}=0.13$ (isohexane:EtOAc, 1:1). HRMS $\left.(E S)^{+}\right): \mathrm{C}_{13} \mathrm{H}_{23} \mathrm{NNaO}_{4} \mathrm{~S}_{2}{ }^{+}[\mathrm{M}+\mathrm{Na}]^{+}$: calc. 344.09607, found 344.09613. ${ }^{1} \mathrm{H}-\mathrm{NMR}\left(400 \mathrm{MHz}, \mathrm{CDCl}_{3}\right): \delta(\mathrm{ppm})=4.58(\mathrm{~d}, J=9.0 \mathrm{~Hz}, 1 \mathrm{H},-\mathrm{NH}), 3.89-3.74(\mathrm{~m}, 1 \mathrm{H}), 3.08(\mathrm{td}$, $J=16.5,15.2,6.0 \mathrm{~Hz}, 2 \mathrm{H}), 2.98(\mathrm{ddd}, J=14.0,8.6,5.5 \mathrm{~Hz}, 1 \mathrm{H}), 2.82(\mathrm{ddd}, J=13.7,8.6,7.0 \mathrm{~Hz}, 1 \mathrm{H}), 2.35(\mathrm{~s}, 3 \mathrm{H}$ ), $2.32(\mathrm{~s}, 3 \mathrm{H}), 1.79$ (dddd, $J=13.5,8.6,7.0,4.7 \mathrm{~Hz}, 1 \mathrm{H}), 1.74-1.63(\mathrm{~m}, 1 \mathrm{H}), 1.43(\mathrm{~s}, 9 \mathrm{H})$.

\section{(S)-N-Boc-1,2-dithian-4-amine (23) ${ }^{22}$}

Prepared from 22 (501 mg, $1.56 \mathrm{mmol}$ ) according to general protocol E. Compound 23 (360 mg, $1.53 \mathrm{mmol}, 98 \%$ ) was obtained as a colourless solid. ${ }^{22}$

TLC $\mathrm{R}_{f}=0.57$ (n-pentane:Et $\left.{ }_{2} \mathrm{O}, 2: 1\right)$. HRMS $\left(\mathrm{ESI}^{+}\right): \mathrm{C}_{9} \mathrm{H}_{17} \mathrm{NO}_{2} \mathrm{~S}_{2}{ }^{+}[\mathrm{M}+\mathrm{H}]^{+}$: calc. 235.0701, found 235.0694. ${ }^{1} \mathrm{H}-\mathrm{NMR}$ $\left(500 \mathrm{MHz}, \mathrm{DMSO}-\mathrm{d}_{6}\right): \delta(\mathrm{ppm})=7.08(\mathrm{~d}, J=8.1 \mathrm{~Hz}, 1 \mathrm{H}), 3.56-3.44(\mathrm{~m}, 1 \mathrm{H}), 3.11-3.01(\mathrm{~m}, 1 \mathrm{H}), 2.95(\mathrm{t}$, $J=12.3 \mathrm{~Hz}, 1 \mathrm{H}), 2.87(\mathrm{dd}, J=12.9,3.2 \mathrm{~Hz}, 1 \mathrm{H}), 2.60(\mathrm{dd}, J=13.0,10.5 \mathrm{~Hz}, 1 \mathrm{H}), 2.06(\mathrm{dd}, J=13.3,3.7 \mathrm{~Hz}, 1 \mathrm{H})$, $1.64(\mathrm{dd}, J=14.4,11.2 \mathrm{~Hz}, 1 \mathrm{H}), 1.38(\mathrm{~s}, 9 \mathrm{H})$.

\section{(S)-N-Boc-N-methyl-1,2-dithian-4-amine (24)}<smiles>CN(C(=O)OC(C)(C)C)C1CCSCC1</smiles>

Prepared from $23(400 \mathrm{mg}, 1.70 \mathrm{mmol})$ according to general protocol D. Compound 24 (301 mg, $1.21 \mathrm{mmol}$, $71 \%$ ) was obtained as a colourless solid after purification by FCC (isohexane/EtOAc).

TLC $R_{f}=0.74$ (n-pentane:Et ${ }_{2} \mathrm{O}, 2: 1$ ). HRMS (EI): $\mathrm{C}_{10} \mathrm{H}_{19} \mathrm{NO}_{2} \mathrm{~S}_{2}$ [M-e]: calc. 249.0852, found 249.0867.

${ }^{1} \mathrm{H}-\mathrm{NMR}\left(400 \mathrm{MHz}, \mathrm{CDCl}_{3}\right): \delta(\mathrm{ppm})=4.48-3.88(\mathrm{~m}, 1 \mathrm{H}, 1-\mathrm{H}), 3.18\left(\mathrm{t}, \mathrm{J}=13.0 \mathrm{~Hz}, 1 \mathrm{H}, 3-\mathrm{H}_{\mathrm{A}}\right), 2.99(\mathrm{t}, \mathrm{J}=15.4 \mathrm{~Hz}$, $\left.2 \mathrm{H}, 2-\mathrm{H}_{\mathrm{A}} ; 3-\mathrm{H}_{\mathrm{B}}\right), 2.76(\mathrm{~s}, 3 \mathrm{H}, 5-\mathrm{H}), 2.66\left(\mathrm{dd}, J=12.8,3.2 \mathrm{~Hz}, 1 \mathrm{H}, 2-\mathrm{H}_{\mathrm{B}}\right), 2.09\left(\mathrm{~d}, J=13.1 \mathrm{~Hz}, 1 \mathrm{H}, 4-\mathrm{H}_{\mathrm{A}}\right), 2.05-$ $1.92\left(\mathrm{~m}, 1 \mathrm{H}, 4-\mathrm{H}_{\mathrm{B}}\right), 1.46(\mathrm{~s}, 9 \mathrm{H}, 8-\mathrm{H}) .{ }^{13} \mathrm{C}-\mathrm{NMR}\left(101 \mathrm{MHz}, \mathrm{CDCl}_{3}\right): \delta(\mathrm{ppm})=155.2(\mathrm{C}=\mathrm{O}, \mathrm{C} 6), 80.2\left(\mathrm{C}\left(\mathrm{CH}_{3}\right)_{3}, \mathrm{C} 7\right)$, 55.9, $53.3(\mathrm{CH}, \mathrm{C} 1), 36.4\left(\mathrm{CH}_{2}, \mathrm{C} 3\right), 36.1\left(\mathrm{CH}_{2}, \mathrm{C} 2\right), 32.9\left(\mathrm{CH}_{2}, \mathrm{C} 4\right), 29.3\left(\mathrm{CH}_{3}, \mathrm{C} 5\right), 28.6\left(\mathrm{C}\left(\mathrm{CH}_{3}\right)_{3}, \mathrm{C} 8\right)$. 


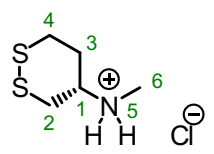

Prepared from $24(92 \mathrm{mg}, 0.37 \mathrm{mmol})$ according to general protocol $\mathbf{F}$ using a larger amount of $\mathrm{HCl}$ (15 eq.). 25 (68 mg, $0.37 \mathrm{mmol}$, quant.) was obtained as a colourless solid.

${ }^{1} \mathrm{H}-\mathrm{NMR}\left(500 \mathrm{MHz}, \mathrm{DMSO}-\mathrm{d}_{6}\right): \delta(\mathrm{ppm})=9.34(\mathrm{~s}, 2 \mathrm{H}, 5-\mathrm{H}), 3.32-3.25(\mathrm{~m}, 2 \mathrm{H}, 1-\mathrm{H} ; 2-\mathrm{H}), 3.16$ (ddd, $J=13.9,5.4$, $\left.3.2 \mathrm{~Hz}, 1 \mathrm{H}, 2-\mathrm{H}_{\mathrm{B}}\right), 3.03-2.90(\mathrm{~m}, 2 \mathrm{H}, 3-\mathrm{H}), 2.53(\mathrm{~s}, 3 \mathrm{H}, 6-\mathrm{H}), 2.45-2.37\left(\mathrm{~m}, 1 \mathrm{H}, 4-\mathrm{H}_{\mathrm{A}}\right), 1.86(\mathrm{q}, J=11.7 \mathrm{~Hz}, 1 \mathrm{H}$, $\left.4-\mathrm{H}_{\mathrm{B}}\right) .{ }^{13} \mathrm{C}-\mathrm{NMR}\left(126 \mathrm{MHz}, \mathrm{DMSO}-\mathrm{d}_{6}\right): \delta(\mathrm{ppm})=55.8(\mathrm{CH}, \mathrm{C} 1), 33.0\left(\mathrm{CH}_{2}, \mathrm{C} 2\right), 32.9\left(\mathrm{CH}_{2}, \mathrm{C} 3\right), 30.0\left(\mathrm{CH}_{2}, \mathrm{C} 4\right)$, $28.9\left(\mathrm{CH}_{3}, \mathrm{C} 6\right)$.

\section{SS60-type precursor: Scale-up route}

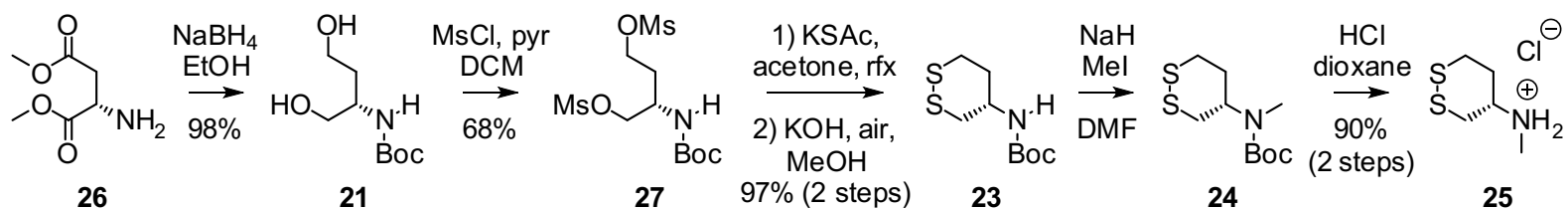

(S)-N-Boc 2-aminobutane-1,4-diol (21)

To a solution of $N$-Boc $L$-aspartic acid dimethyl ester 26 (17.4 g, $66.6 \mathrm{mmol}, 1.0$ eq.) in anhydrous EtOH (260 mL) at $0{ }^{\circ} \mathrm{C}$ was carefully added $\mathrm{NaBH}_{4}(20.2 \mathrm{~g}, 533 \mathrm{mmol}, 8 \mathrm{eq}$.) in portions, the resulting mixture was allowed to warm to r.t. and further stirred for $15 \mathrm{~h}$. Then, the mixture was heated to reflux for $3 \mathrm{~h}$. After cooling to r.t., sat. aq. $\mathrm{NaCl}$ $(250 \mathrm{~mL})$ was added, the mixture was stirred for several minutes and filtered. The solids were washed with $\mathrm{Et}_{2} \mathrm{O}$ $(4 \times 50 \mathrm{~mL})$ and discarded. The layers were separated and the aq. layer was extracted with additional $\mathrm{Et}_{2} \mathrm{O}$ $(2 \times 75 \mathrm{~mL})$. The combined organic layers were dried over $\mathrm{MgSO}_{4}$, filtered and the solvent was removed under reduced pressure. After azeotropic drying of the residue with toluene, $21(13.4 \mathrm{~g}, 65.3 \mathrm{mmol}, 98 \%)$ was obtained as a white solid.

\section{(S)-N-Boc 2-aminobutane-1,4-diyl dimethanesulfonate (27)}

To a mixture of 21 (4.86 g, $23.7 \mathrm{mmol}, 1.0$ eq.), anhydrous DCM (50 mL) and pyridine (9.56 mL, $118 \mathrm{mmol}, 5.0$ eq.) at $0{ }^{\circ} \mathrm{C}$ was dropwise added $\mathrm{MsCl}(4.03 \mathrm{~mL}, 52.1 \mathrm{mmol}$. 2.2 eq.). The mixture was warmed to r.t. and was further stirred at r.t. for $15 \mathrm{~h}$, was then diluted with $\mathrm{DCM}(25 \mathrm{~mL})$, washed with $2 \mathrm{M}$ aq. $\mathrm{HCl}(100 \mathrm{~mL}$ and $50 \mathrm{~mL})$ and water $(50 \mathrm{~mL})$, dried over $\mathrm{MgSO}_{4}$, filtered and concentrated under reduced pressure. The residue was suspended in DCM (50 mL) and filtered. The solids were dried to obtain $27(3.30 \mathrm{~g}, 8.85 \mathrm{mmol}, 37 \%)$ as a white solid. The filtrate was diluted with toluene $(25 \mathrm{~mL})$, the $\mathrm{DCM}$ was evaporated under reduced pressure and the remainder kept at r.t. for $1 \mathrm{~h}$. The resulting precipitate was collected, washed with toluene $(25 \mathrm{ml}$ and $10 \mathrm{~mL})$ followed by a small amount of hexane and dried to afford additional $27(2.64 \mathrm{~g}, 7.30 \mathrm{mmol}, 31 \%)$ as an off-white solid.

\section{(S)-N-Boc 1,2-dithian-4-amine (23)}

Step 1: A mixture of 27 (3.00 g, $8.30 \mathrm{mmol}, 1.0$ eq.), KSAc (2.84 g, $24.9 \mathrm{mmol}, 3.0$ eq.) and acetone (85 mL) was heated to reflux for $2 \mathrm{~h}$. Then, all volatiles were evaporated under reduced pressure. DCM $(75 \mathrm{~mL})$ and sat. aq. $\mathrm{NaHCO}_{3}(75 \mathrm{~mL})$ were added to the residue, the layers were separated and the aqueous layer was extracted with additional DCM $(2 \times 25 \mathrm{~mL})$. The combined organic layers were washed with water $(100 \mathrm{~mL})$, dried over $\mathrm{MgSO}_{4}$, filtered and the solvent was removed under reduced pressure. The residue was directly used without purification in the next step.

Step 2: The material obtained in step 1 was dissolved in a $0.15 \mathrm{M}$ solution of $\mathrm{KOH}$ in $\mathrm{MeOH}(170 \mathrm{~mL})$. After stirring open to air for $15 \mathrm{~h}, \mathrm{AcOH}(1.53 \mathrm{~mL}, 26.8 \mathrm{mmol})$ was added, all volatiles were evaporated under reduced pressure and the residue was taken up in $\mathrm{EtOAc}(100 \mathrm{~mL})$ and sat. aq. $\mathrm{NaCl}(60 \mathrm{~mL})$. The layers were separated and the aq. layer was extracted with EtOAc $(30 \mathrm{~mL})$. The combined organic layers were dried over $\mathrm{MgSO}_{4}$, filtered and evaporated under reduced pressure. The residue was dissolved in EtOAc $(10 \mathrm{~mL})$ and hexane $(30 \mathrm{~mL})$ was added. The solution was filtered over silica $(6 \mathrm{~g})$ and the silica was washed with additional isohexane/EtOAc $(3: 1,80 \mathrm{~mL})$. Removal of the solvent under reduced pressure afforded $23(1.89 \mathrm{~g}, 8.03 \mathrm{mmol}, 97 \%$ over 2 steps) as a faint yellow solid.

\section{(S)-N-methyl-1,2-dithian-4-amine hydrochloride (25)}

Step 1: To a mixture of $60 \% \mathrm{NaH}$ mineral oil suspension $(0.408 \mathrm{mg}, 10.2 \mathrm{mmol}, 1.5 \mathrm{eq}$.) and anhydrous DMF $(25 \mathrm{~mL})$ at $0^{\circ} \mathrm{C}$ under nitrogen atmosphere was dropwise added a solution of $23(1.60 \mathrm{~g}, 6.8 \mathrm{mmol}, 1.0 \mathrm{eq}$.) in anhydrous DMF ( $5 \mathrm{~mL})$. After stirring for $30 \mathrm{~min}$, Mel $(635 \mu \mathrm{L}, 10.2 \mathrm{mmol}, 1.5 \mathrm{eq}$.) was added and stirring was continued for $4 \mathrm{~h}$ with warming to r.t., before the mixture was carefully poured into sat. aq. $\mathrm{NH}_{4} \mathrm{Cl}(60 \mathrm{~mL})$ and extracted with EtOAc $(3 \times 40 \mathrm{~mL})$. The organic extracts were washed with water $(2 \times 60 \mathrm{~mL})$ and sat. aq. $\mathrm{NaCl}$ 
$(60 \mathrm{~mL})$, dried over $\mathrm{MgSO}_{4}$, filtered and the solvent was removed under reduced pressure. The residue containing 24 was directly used without purification in the next step.

Step 2: To the material obtained in step 1 was added a $4 \mathrm{M}$ solution of $\mathrm{HCl}$ in dioxane $(7.5 \mathrm{~mL})$. The mixture was stirred for $3.5 \mathrm{~h}$, before $\mathrm{Et}_{2} \mathrm{O}(30 \mathrm{~mL})$ was added and the mixture was filtered. The solids were washed with $\mathrm{Et}_{2} \mathrm{O}$ $(2 \times 15 \mathrm{~mL})$ and dried to afford $25(1.20 \mathrm{~g}, 6.46 \mathrm{mmol}, 90 \%$ over 2 steps $)$ as a faint brownish-grey solid.

\section{Inverted SS60-type precursor}

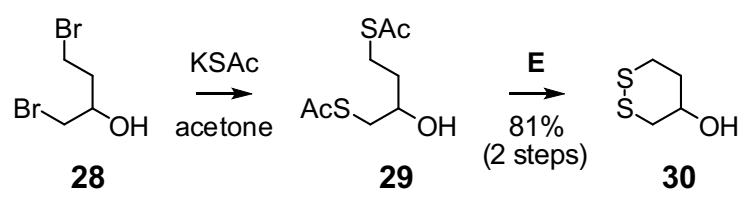

\section{1,2-dithian-4-ol (30)}<smiles>CC1CCCSS1</smiles>

Step 1: To a mixture of KSAc $(2.95 \mathrm{~g}, 25.9 \mathrm{mmol}, 3.0$ eq.) and acetone $(85 \mathrm{~mL})$ under nitrogen atmosphere was added 1,4-dibromo-2-butanol $28(2.00 \mathrm{~g}, 8.62 \mathrm{mmol}, 1.0$ eq.). The mixture was stirred in the dark for $15 \mathrm{~h}$, then filtered. The solids were washed with acetone and discarded. The filtrate was evaporated under reduced pressure and the residue was taken up in $\mathrm{DCM}(75 \mathrm{~mL})$ and sat. aq. $\mathrm{NaHCO}_{3}(75 \mathrm{~mL})$. The layers were separated and the aq. layer was extracted with DCM $(2 \times 25 \mathrm{~mL})$. The combined organic layers were washed with water $(100 \mathrm{~mL})$, dried over $\mathrm{MgSO}_{4}$, filtered and the solvent was removed under reduced pressure. The residue containing 29 was directly used without purification in the next step.

Step 2: To the material obtained in step 1 were added $\mathrm{MeOH}(170 \mathrm{~mL})$ and $\mathrm{KOH}(1.43 \mathrm{~g}, 25.5 \mathrm{mmol})$. The mixture was stirred open to air for $15 \mathrm{~h}$, before $\mathrm{AcOH}(1.53 \mathrm{~mL}, 26.8 \mathrm{mmol})$ was added and all volatiles were removed under reduced pressure. The residue was suspended in DCM $(10 \mathrm{~mL})$ and filtered. The solids were washed with additional DCM $(2 \times 10 \mathrm{~mL})$ and discarded. The filtrate was evaporated under reduced pressure and the residue was purified by FCC (isohexane:EtOAc, 2:1) to afford 30 (951 mg, $6.98 \mathrm{mmol}, 81 \%)$ as an off-white solid.

TLC $\mathrm{R}_{f}=0.41$ (isohexane:EtOAc, 1:1). HRMS (ESI): $\mathrm{C}_{4} \mathrm{H}_{7} \mathrm{OS}_{2}^{-}[\mathrm{M}-\mathrm{H}]^{-}$calc. 134.99438, found 134.99247.

${ }^{1} \mathrm{H}-N M R(400 \mathrm{MHz}$, DMSO-d 6 ): $\delta(\mathrm{ppm})=5.18(\mathrm{~d}, J=5.0 \mathrm{~Hz}, 1 \mathrm{H}, 5-\mathrm{H}), 3.54(\mathrm{td}, J=10.0,5.4 \mathrm{~Hz}, 1 \mathrm{H}, 1-\mathrm{H}), 3.05$ (dt, $\left.J=13.8,4.1 \mathrm{~Hz}, 1 \mathrm{H}, 2-\mathrm{H}_{\mathrm{A}}\right), 2.98-2.85\left(\mathrm{~m}, 2 \mathrm{H}, 2-\mathrm{H}_{\mathrm{B}}, 4-\mathrm{H}_{\mathrm{A}}\right), 2.56\left(\mathrm{dd}, J=12.9,10.0 \mathrm{~Hz}, 1 \mathrm{H}, 4-\mathrm{H}_{\mathrm{B}}\right), 2.16(\mathrm{~d}$, $\left.J=13.4 \mathrm{~Hz}, 1 \mathrm{H}, 3-\mathrm{H}_{\mathrm{B}}\right), 1.60-1.44\left(\mathrm{~m}, 1 \mathrm{H}, 3-\mathrm{H}_{\mathrm{B}}\right) .{ }^{13} \mathrm{C}-\mathrm{NMR}\left(101 \mathrm{MHz}, \mathrm{DMSO}-\mathrm{d}_{6}\right): \delta(\mathrm{ppm})=67.1(\mathrm{CH}, \mathrm{C} 1)$, $39.2\left(\mathrm{CH}_{2}, \mathrm{C} 2\right), 36.8\left(\mathrm{CH}_{2}, \mathrm{C} 4\right), 34.3\left(\mathrm{CH}_{2}, \mathrm{C} 3\right)$. 
Symmetric linear disulfide SS00-type probe precursor

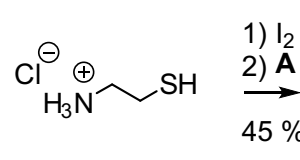

31

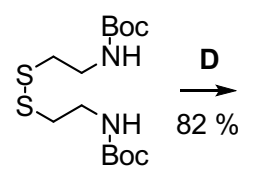

32

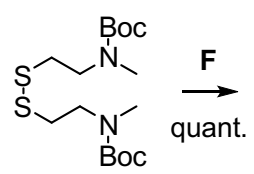

33

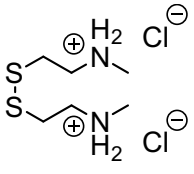

34

$N$-Boc, $N$ '-Boc 2,2'-disulfanediylbis(ethan-1-amine) (32) ${ }^{24}$

Step 1: To a solution of cysteamine hydrochloride $31(1.00 \mathrm{~g}, 8.80 \mathrm{mmol})$ and $\mathrm{KOH}(1.16 \mathrm{~g}, 17.6 \mathrm{mmol})$ in $\mathrm{MeOH}$ $(30 \mathrm{~mL})$ was dropwise added a $0.5 \mathrm{M}$ solution of $\mathrm{I}_{2}$ in $\mathrm{MeOH}$ until a permanently coloured solution was obtained. The volatiles were removed under reduced pressure and sat. aq. $\mathrm{NaCl}$ and a small amount of aq. $\mathrm{Na}_{2} \mathrm{~S}_{2} \mathrm{O}_{3} \mathrm{were}$ added. The mixture was extracted with $\mathrm{DCM}$, dried over $\mathrm{Na}_{2} \mathrm{SO}_{4}$, filtered and concentrated under reduced pressure. The residue was directly in the next step without further purification.

Step 2: The material obtained in step 1 was treated according to general protocol A. Compound $\mathbf{3 2}$ with minor impurities $(0.70 \mathrm{~g}, 2.00 \mathrm{mmol}, 45 \%)$ was obtained as colourless solid after FCC ( $n$-pentane/Et $\left.{ }_{2} \mathrm{O}\right)$. A pure sample of 32 was obtained by recrystallization from methanol. ${ }^{24}$

TLC $\mathrm{R}_{f}=0.29$ (isohexane:EtOAc, 3:1). HRMS $\left(E S I^{+}\right): \mathrm{C}_{14} \mathrm{H}_{28} \mathrm{NNa}_{2} \mathrm{O}_{4} \mathrm{~S}_{2}{ }^{+}[\mathrm{M}+\mathrm{Na}]^{+}$: calc. 375.13827, found 375.13825. ${ }^{1} \mathrm{H}-\mathrm{NMR}\left(400 \mathrm{MHz}, \mathrm{CDCl}_{3}\right): \delta(\mathrm{ppm})=5.01(\mathrm{~s}, 2 \mathrm{H}), 3.45(\mathrm{q}, J=6.3 \mathrm{~Hz}, 4 \mathrm{H}), 2.79(\mathrm{t}, J=6.4 \mathrm{~Hz}, 4 \mathrm{H})$, $1.45(\mathrm{~s}, 18 \mathrm{H}) .{ }^{13} \mathrm{C}-\mathrm{NMR}\left(101 \mathrm{MHz}, \mathrm{CDCl}_{3}\right): \delta(\mathrm{ppm}) 155.8(\mathrm{C}=\mathrm{O}), 79.6\left(\mathrm{C}_{\left.\left(\mathrm{CH}_{3}\right)_{3}\right),}, 39.3\left(\mathrm{CH}_{2}\right), 38.5\left(\mathrm{CH}_{2}\right), 28.4\right.$ $\left(\mathrm{C}\left(\mathrm{CH}_{3}\right)_{3}\right)$.

$N$-Boc, $N$ '-Boc 2,2'-disulfanediylbis( $N$-methylethan-1-amine) (33)<smiles>CCCCC(C)N(C)C(=O)OC(C)(C)C</smiles>

Prepared from $32(0.78 \mathrm{~g}, 2.21 \mathrm{mmol})$ according to general protocol D. Compound $33(0.69 \mathrm{~g}, 1.82 \mathrm{mmol}, 82 \%)$ was obtained as a colourless solid after FCC (isohexane/EtOAc).

TLC $R_{f}=0.5$ (isohexane:EtOAc, 3:1). HRMS $\left(E S I^{+}\right): \mathrm{C}_{16} \mathrm{H}_{32} \mathrm{~N}_{2} \mathrm{O}_{4} \mathrm{~S}_{2} \mathrm{Na}^{+}:[\mathrm{M}+\mathrm{Na}]^{+}$calc. 403.16957, found 403.16964. ${ }^{1} \mathrm{H}-\mathrm{NMR}\left(400 \mathrm{MHz}, \mathrm{CDCl}_{3}\right): \delta(\mathrm{ppm})=3.50(\mathrm{t}, J=7.2 \mathrm{~Hz}, 4 \mathrm{H}, 1-\mathrm{H}), 2.89(\mathrm{~s}, 6 \mathrm{H}, 3-\mathrm{H}), 2.81(\mathrm{~s}, 4 \mathrm{H}$, 2-H), $1.46(\mathrm{~s}, 18 \mathrm{H}, 6-\mathrm{H}) .{ }^{13} \mathrm{C}-\mathrm{NMR}(101 \mathrm{MHz}, \mathrm{CDCl} 3): \delta(\mathrm{ppm})=155.4(\mathrm{C}=\mathrm{O}, \mathrm{C} 4), 79.6\left(\mathrm{C}\left(\mathrm{CH}_{3}\right)_{3}, \mathrm{C} 5\right), 48.3\left(\mathrm{CH}_{2}\right.$,

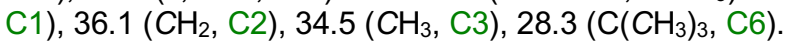

\section{2,2'-disulfanediylbis( $N$-methylethan-1-amine) dihydrochloride (34)}

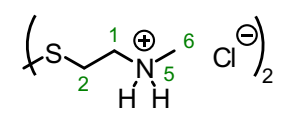

Prepared from $33(0.69 \mathrm{mg}, 1.82 \mathrm{mmol})$ according to general protocol $\mathbf{F}$ using a larger amount of $\mathrm{HCl}$ (15 eq.). $34(0.46 \mathrm{mg}, 1.82 \mathrm{mmol}$, quant.) was obtained as a colourless solid.

${ }^{1} \mathrm{H}-\mathrm{NMR}\left(400 \mathrm{MHz}, \mathrm{DMSO}-\mathrm{d}_{6}\right): \delta(\mathrm{ppm})=9.27(\mathrm{~s}, 4 \mathrm{H}, 5-\mathrm{H}), 3.19(\mathrm{~d}, J=7.4 \mathrm{~Hz}, 4 \mathrm{H}, 1-\mathrm{H}), 3.09(\mathrm{dd}, J=8.1,5.7 \mathrm{~Hz}$, $4 \mathrm{H}, 2-\mathrm{H}), 2.56(\mathrm{~s}, 6 \mathrm{H}, 6-\mathrm{H}) .{ }^{13} \mathrm{C}-\mathrm{NMR}\left(101 \mathrm{MHz}, \mathrm{DMSO}-\mathrm{d}_{6}\right): \delta(\mathrm{ppm})=47.0\left(\mathrm{CH}_{2}, \mathrm{C} 1\right), 32.4\left(\mathrm{CH}_{2}, \mathrm{C} 2\right), 32.3\left(\mathrm{CH}_{3}\right.$, C6).

\section{Unsymmetric linear disulfide SSOOM-type precursor}

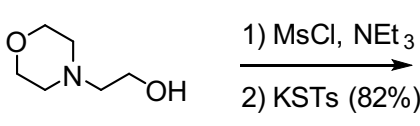

35<smiles>Cc1ccc(S(=O)(=O)CCN2CCOCC2)cc1</smiles>

36<smiles>CNCCO</smiles>

37

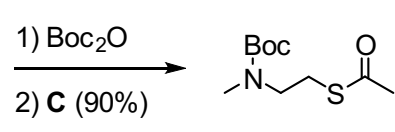

38<smiles>CNCCSSCCN1CCOCC1</smiles> 


\section{S-(2-morpholinoethyl) 4-methylbenzenesulfonothioate (36)}

Step 1: $\mathrm{Et}_{3} \mathrm{~N}(5.58 \mathrm{~mL}, 40.0 \mathrm{mmol})$ was added to a solution of 2-morpholinoethanol 35 (3.50 g, $\left.26.7 \mathrm{mmol}\right)$ in anhydrous DCM $(100 \mathrm{~mL})$ under $\mathrm{N}_{2}$-atmosphere. The mixture was cooled to $0^{\circ} \mathrm{C}$ and $\mathrm{MsCl}(2.48 \mathrm{~mL}, 32.0 \mathrm{mmol})$ was added dropwise. After stirring for $15 \mathrm{~min}$, the mixture was washed with sat. aq. $\mathrm{NaHCO}_{3}(100 \mathrm{~mL})$ and dried over $\mathrm{Na}_{2} \mathrm{SO}_{4}$ and filtered. The volatiles were evaporated under reduced pressure and the residue was used in the next step without purification.

Step 2: To the material obtained in step 1 were added acetone $(100 \mathrm{~mL})$ and potassium $p$-toluenethiosulfonate (KSTs) $(6.04 \mathrm{~g}, 26.7 \mathrm{mmol})$. The mixture was warmed to reflux for $1.25 \mathrm{~h}$, cooled to r.t., further stirred for $15 \mathrm{~h}$ and before being filtered. The solids were washed with additional acetone and discarded. The filtrate was concentrated under reduced pressure to remove all acetone and the residue was taken up in EtOAc $(50 \mathrm{~mL})$. The mixture was filtered over silica $(15 \mathrm{~g})$ and the silica was washed with additional EtOAc $(75 \mathrm{~mL})$. The volatiles were removed under reduced to obtain thiosulfonate 36 as a yellowish oil $(6.59 \mathrm{~g}, 21.9 \mathrm{mmol}, 82 \%$ over 2 steps), which was used without further purification.

${ }^{1} \mathrm{H}-\mathrm{NMR}\left(400 \mathrm{MHz}, \mathrm{CDCl}_{3}\right): \delta(\mathrm{ppm})=7.81(\mathrm{~d}, \mathrm{~J}=8.4 \mathrm{~Hz}, 2 \mathrm{H}), 7.34(\mathrm{~d}, J=8.2 \mathrm{~Hz}, 2 \mathrm{H}), 3.67-3.62(\mathrm{~m}, 4 \mathrm{H}), 3.13$ $(\mathrm{t}, J=6.9 \mathrm{~Hz}, 2 \mathrm{H}), 2.61(\mathrm{t}, J=6.9 \mathrm{~Hz}, 2 \mathrm{H}), 2.45(\mathrm{~s}, 3 \mathrm{H}), 2.42-2.36(\mathrm{~m}, 4 \mathrm{H})$.

The spectroscopic data matched previously reported values. ${ }^{25}$

\section{S-(2-((tert-butoxycarbonyl)(methyl)amino)ethyl) ethanethioate (38)}

Step 1: 2-methylaminoethanol $37(2.82 \mathrm{~g}, 37.6 \mathrm{mmol})$ was dissolved in THF $(20 \mathrm{~mL})$ and placed in water bath at r.t.. $\mathrm{Boc}_{2} \mathrm{O}(8.21 \mathrm{~g}, 37.6 \mathrm{mmol})$ was weighed into in a vial, molten and dropwise added to the solution of 2-methylaminoethanol. Then, the remaining material in the vial was dissolved in THF $(5 \mathrm{~mL})$ and also added to the reaction mixture. After stirring at r. t. for $1.5 \mathrm{~h}$, the volatiles were evaporated and the residue was taken forward to the next step without purification.

Step 2: A solution of material obtained in step $1(37.6 \mathrm{mmol})$ was treated according to general procedure $\mathbf{C}$. The volatiles were removed under reduced pressure and the residue was suspended in $\mathrm{Et}_{2} \mathrm{O}(50 \mathrm{~mL})$, followed by the addition of isohexane $(100 \mathrm{~mL})$. The mixture was filtered, the solids were discarded, the filtrate was concentrated under reduced pressure and hexanes $(100 \mathrm{~mL})$ were added to the residue. The mixture was again filtered and the solids were discarded. Finally, the filtrate was concentrated under reduced pressure and the residue was purified by FCC (isohexane:EtOAc) to obtain compound 38 as a faint yellow oil $(7.50 \mathrm{~g}, 32.1 \mathrm{mmol}, 90 \%)$.

TLC $R_{f}=0.39$ (isohexane:EtOAc, 6:1). ${ }^{1} \mathrm{H}-\mathrm{NMR}\left(400 \mathrm{MHz}, \mathrm{CDCl}_{3}\right): \delta(\mathrm{ppm})=3.35(\mathrm{t}, \mathrm{J}=7.1 \mathrm{~Hz}, 2 \mathrm{H}), 3.00(\mathrm{t}$, $J=6.9 \mathrm{~Hz}, 2 \mathrm{H}), 2.89(\mathrm{~s}, 3 \mathrm{H}), 2.34(\mathrm{~s}, 3 \mathrm{H}), 1.46(\mathrm{~s}, 9 \mathrm{H})$.

The spectroscopic data matched previously reported values. ${ }^{26}$

\section{$\mathbf{N}$-methyl-2-((2-morpholinoethyl)disulfaneyl)ethan-1-amine dihydrochloride (40)}

Step 1: A degassed solution of thiosulfonate 36 (5.68 g, $18.9 \mathrm{mmol})$ and thioacetate $38(4.00 \mathrm{~g}, 17.1 \mathrm{mmol})$ in anhydrous $\mathrm{MeOH}(160 \mathrm{~mL})$ under $\mathrm{N}_{2}$-atmosphere was added $\mathrm{K}_{2} \mathrm{CO}_{3}(2.37 \mathrm{~g}, 17.1 \mathrm{mmol})$ and mixture was degassed again. After stirring for $2.5 \mathrm{~h}$, the volatiles were evaporated under reduced pressure, the residue was suspended in EtOAc $(40 \mathrm{~mL})$ and the mixture was filtered. The solids were washed with a small amount additional EtOAc and discarded. The filtrate was concentrated under reduced pressure and the residue was partially purified by FCC (isohexane:EtOAc, $\left.R_{f}=0.37\right)$. Two fractions of disulfide $39\left(R_{f}=0.37(E t O A c)\right.$ ) were obtained. Both fractions contained small amounts of unidentified impurities and one fraction additionally a significant contamination by remaining excess thiosulfonate 36 . The two fractions were carried forward separately without further purification.

Step 2: To the almost pure fraction $(2.54 \mathrm{~g})$ of disulfide 39 from the preceding step was added a $4 \mathrm{M}$ solution of $\mathrm{HCl}$ in 1,4-dioxane $(8 \mathrm{~mL})$. The mixture was stirred for $1 \mathrm{~h}$, diluted with $\mathrm{Et}_{2} \mathrm{O}(40 \mathrm{~mL})$ and filtered. The solids were washed with additional $\mathrm{Et}_{2} \mathrm{O}$ and the filtrate was discarded. The solids were suspended in isopropanol $(30 \mathrm{~mL})$ and the mixture was warmed to reflux. After cooling back to r.t., the mixture was diluted with isohexane $(60 \mathrm{~mL})$ and filtered again. The filtrate was discarded and the solids were dried to obtain dihydrochloride $40(2.11 \mathrm{~g}, 6.82 \mathrm{mmol}$, $40 \%$ over two steps) as a white powder. The second fraction $(2.66 \mathrm{~g})$ of disulfide 39 was treated identically to afforded additional 40 (1.97 g, $6.37 \mathrm{mmol}, 37 \%$ over two steps), also in the form of a white powder.

The material that was obtained did not consist only of dihydrochloride $\mathbf{X 5 6}$, but also contained a fraction of the corresponding monohydrochloride. This was observed during NMR spectroscopy, as several signals in the ${ }^{1} \mathrm{H}-\mathrm{NMR}$ spectrum were significantly broadened. To obtain pure dihydrochloride X56 for characterization by NMR spectroscopy, a sample was dissolved in a solution of $\mathrm{HCl}$ in methanol (1.25 $\mathrm{M}$ solution), the solution was evaporated and the residue was dissolved in deuterium oxide. Acetonitrile was added and the signal of the methyl group was used as an internal reference ( $\left.{ }^{1} \mathrm{H}-\mathrm{NMR}: 2.06 \mathrm{ppm},{ }^{13} \mathrm{C}-\mathrm{NMR}: 1.47\right) .{ }^{27}$

HRMS $\left(\mathrm{ESI}^{+}\right): \mathrm{C}_{9} \mathrm{H}_{21} \mathrm{~N}_{2} \mathrm{OS}_{2}{ }^{+}:[\mathrm{M}+\mathrm{H}]^{+}$calc. 237.10898, found 237.10918. ${ }^{1} \mathrm{H}-\mathrm{NMR}(400 \mathrm{MHz}, \mathrm{D} 2 \mathrm{O}): \delta(\mathrm{ppm})=4.13$ (dd, $J=13.2,3.4 \mathrm{~Hz}, 2 \mathrm{H}$ ), 3.86 (ddd, $J=13.7,11.8,2.2 \mathrm{~Hz}, 2 \mathrm{H}), 3.70-3.52(\mathrm{~m}, 4 \mathrm{H}), 3.49-3.40(\mathrm{~m}, 2 \mathrm{H}), 3.28$ $(\mathrm{td}, J=12.6,3.7 \mathrm{~Hz}, 2 \mathrm{H}), 3.22-3.11(\mathrm{~m}, 2 \mathrm{H}), 3.07(\mathrm{t}, J=6.5 \mathrm{~Hz}, 2 \mathrm{H}), 2.77(\mathrm{~s}, 3 \mathrm{H}) .{ }^{13} \mathrm{C}-\mathrm{NMR}\left(100 \mathrm{MHz} \mathrm{D}_{2} \mathrm{O}\right)$ : $\delta(\mathrm{ppm})=64.2,56.0,52.4,47.6,33.4,32.7,30.1$. 
<smiles>OCC1CCCCN1</smiles><smiles></smiles>

41<smiles>COC(=O)N1CCCCC1C(=O)O</smiles>

42

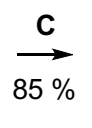<smiles>CC(C)(C)O[Mg]</smiles>

43<smiles>CC(C)(C)OC(=O)[N+]1(C(=O)OCc2ccccc2)CCCCC1CSSCC1CCCCN1C(=O)O</smiles>

44<smiles></smiles>

45

N-Boc piperidin-2-ylmethanol $(42)^{19}$

Prepared from 2-piperidinylmethanol $41(1.00 \mathrm{~g}, 8.68 \mathrm{mmol})$ according to general protocol A. Instead of a dioxane and water mixture the reaction was performed in a $4 / 3$ mixture of THF/water at a concentration of $0.025 \mathrm{M}$ and with $\mathrm{K}_{2} \mathrm{CO}_{3}$ (3.0 eq.) replacing $\mathrm{Et}_{3} \mathrm{~N}$ as the base. $42(1.69 \mathrm{~g}, 7.85 \mathrm{mmol}, 90 \%)$ was obtained as a crystalline solid after recrystallization from $\mathrm{MeOH}: \mathrm{Et}_{2} \mathrm{O}(1: 3){ }^{19}$

TLC $\mathrm{R}_{f}=0.50$ (isohexane:EtOAc, 1:1). HRMS (El): $\mathrm{C}_{11} \mathrm{H}_{21} \mathrm{NO}_{3}$ [M-e]: calc. 215.1516, found 215.1513. ${ }^{1} \mathrm{H}-\mathrm{NMR}$ $\left(400 \mathrm{MHz}, \mathrm{CDCl}_{3}\right): \delta(\mathrm{ppm})=4.25(\mathrm{dq}, J=6.0,3.1 \mathrm{~Hz}, 1 \mathrm{H}), 3.91(\mathrm{~d}, J=13.6 \mathrm{~Hz}, 1 \mathrm{H}), 3.76(\mathrm{dd}, J=10.9,8.8 \mathrm{~Hz}$, $1 \mathrm{H}), 3.58(\mathrm{dd}, J=11.0,6.2 \mathrm{~Hz}, 1 \mathrm{H}), 2.83(\mathrm{t}, J=12.2 \mathrm{~Hz}, 1 \mathrm{H}), 2.47(\mathrm{~s}, 1 \mathrm{H}), 1.71-1.63(\mathrm{~m}, 1 \mathrm{H}), 1.62-1.51(\mathrm{~m}$, $3 \mathrm{H}), 1.46-1.32(\mathrm{~m}, 2 \mathrm{H}), 1.43(\mathrm{~s}, 9 \mathrm{H})$.

\section{$N$-Boc (S)-(piperidin-2-ylmethyl) ethanethioate (43) ${ }^{28}$}

Prepared from $42(1.62 \mathrm{~g}, 7.45 \mathrm{mmol})$ according to general protocol C. A different concentration than in the general protocol was employed: $0.18 \mathrm{M}$ for the $\mathrm{PPh}_{3}$ solution. $43(1.74 \mathrm{~g}, 6.35 \mathrm{mmol}, 85 \%)$ was obtained as a colourless solid after FCC (isohexane/EtOAc). ${ }^{28}$

TLC $R_{f}=0.59$ (isohexane:EtOAc, 2:1). HRMS $\left(\mathrm{ESI}^{+}\right): \mathrm{C}_{13} \mathrm{H}_{23} \mathrm{NNaO}_{3} \mathrm{~S}^{+}:[\mathrm{M}+\mathrm{Na}]^{+}$calc. 296.12909, found 296.12909. ${ }^{1} \mathrm{H}-N M R\left(400 \mathrm{MHz}, \mathrm{CDCl}_{3}\right): \delta(\mathrm{ppm})=4.35(\mathrm{~s}, 1 \mathrm{H}), 4.00(\mathrm{~d}, J=13.8 \mathrm{~Hz}, 1 \mathrm{H}), 3.18(\mathrm{dd}, J=13.5,8.5 \mathrm{~Hz}, 1 \mathrm{H}), 3.09$ $(\mathrm{dd}, J=13.6,7.2 \mathrm{~Hz}, 1 \mathrm{H}), 2.77(\mathrm{t}, J=13.5 \mathrm{~Hz}, 1 \mathrm{H}), 2.34(\mathrm{~s}, 3 \mathrm{H}), 1.76-1.53(\mathrm{~m}, 4 \mathrm{H}), 1.47(\mathrm{~s}, 9 \mathrm{H}), 1.47-1.32(\mathrm{~m}$, $2 \mathrm{H})$.

\section{N-Boc, $N$ '-Boc 1,2-bis(piperidin-2-ylmethyl)disulfane (44)}

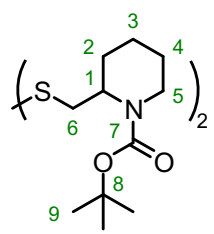

Prepared from $43(1.050 \mathrm{~g}, 3.84 \mathrm{mmol})$ according general protocol $\mathbf{E}$ using a methanolic solution of $\mathrm{KOH}$ (1.00 mL, $0.015 \mathrm{M}) .44(801 \mathrm{mg}, 1.74 \mathrm{mmol}, 91 \%)$ was obtained as a colourless solid.

TLC $\mathrm{R}_{f}=0.46$ (isohexane:EtOAc, 5:1). HRMS (ESI ${ }^{+}$: $\mathrm{C}_{22} \mathrm{H}_{41} \mathrm{~N}_{2} \mathrm{O}_{2} \mathrm{~S}_{2}{ }^{+}:[\mathrm{M}+\mathrm{H}]^{+}$: calc. 461.25023, found 461.25020.

${ }^{1} \mathrm{H}-\mathrm{NMR}\left(400 \mathrm{MHz}, \mathrm{CDCl}_{3}\right): \delta(\mathrm{ppm})=4.47(\mathrm{~s}, 2 \mathrm{H}, 1-\mathrm{H}), 4.01\left(\mathrm{~d}, \mathrm{~J}=13.6 \mathrm{~Hz}, 2 \mathrm{H}, 5-\mathrm{H}_{\mathrm{A}}\right), 3.00-2.79(\mathrm{~m}, 4 \mathrm{H}, 6-\mathrm{H})$, $2.72\left(\mathrm{t}, J=12.5 \mathrm{~Hz}, 2 \mathrm{H}, 5-\mathrm{H}_{\mathrm{B}}\right), 1.85\left(\mathrm{dd}, J=13.7,3.1 \mathrm{~Hz}, 2 \mathrm{H}, 4-\mathrm{H}_{\mathrm{A}}\right), 1.67-1.58\left(\mathrm{~m}, 6 \mathrm{H}, 2-\mathrm{H}, 4-\mathrm{H}_{\mathrm{B}}\right), 1.46(\mathrm{~s}, 18 \mathrm{H}$,

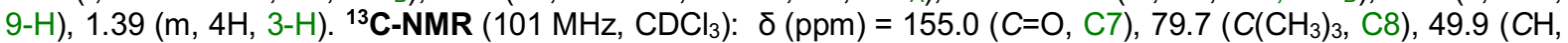
C1), $39.3\left(\mathrm{CH}_{2}, \mathrm{C} 5\right), 38.9\left(\mathrm{CH}_{2}, \mathrm{C} 6\right), 28.6\left(\mathrm{C}_{\left.\left(\mathrm{CH}_{3}\right)_{3}, \mathrm{C}\right)}\right), 26.8\left(\mathrm{CH}_{2}, \mathrm{C} 4\right), 25.5\left(\mathrm{CH}_{2}, \mathrm{C} 2\right), 19.1\left(\mathrm{CH}_{2}, \mathrm{C} 3\right)$.

\section{1,2-bis(piperidin-2-ylmethyl)disulfane dihydrochloride (45)}

$$
\text { (s } \underbrace{1}_{7} \overbrace{\substack{\mathrm{H}^{\prime} \mathrm{H} \\ N_{6}^{-}}}^{3} \mathrm{Cl}^{\Theta})_{2}
$$

Prepared from $44(714 \mathrm{mg}, 1.55 \mathrm{mmol})$ according to general protocol $\mathbf{F}$. A larger amount of $\mathrm{HCl}(15$ eq.) than in the general protocol was employed. $45(513 \mathrm{mg}, 1.54 \mathrm{mmol}, 99 \%)$ was obtained as a colourless crystalline solid.

${ }^{1} \mathrm{H}-N M R\left(400 \mathrm{MHz}, \mathrm{DMSO}-\mathrm{d}_{6}\right): \delta(\mathrm{ppm})=9.37(\mathrm{~s}, 4 \mathrm{H}, 6-\mathrm{H}), 3.33-3.29(\mathrm{~m}, 2 \mathrm{H}, 1-\mathrm{H}), 3.28-3.17\left(\mathrm{~m}, 4 \mathrm{H}, 5-\mathrm{H}_{\mathrm{A}}\right.$; $\left.7-\mathrm{H}_{\mathrm{A}}\right), 3.01\left(\mathrm{ddd}, J=19.4,13.8,7.7 \mathrm{~Hz}, 2 \mathrm{H}, 7-\mathrm{H}_{\mathrm{B}}\right), 2.91\left(\mathrm{~d}, J=10.9 \mathrm{~Hz}, 2 \mathrm{H}, 5-\mathrm{H}_{\mathrm{B}}\right), 2.00\left(\mathrm{~d}, J=7.6 \mathrm{~Hz}, 2 \mathrm{H}, 2-\mathrm{H}_{\mathrm{A}}\right)$, $1.82-1.58\left(\mathrm{~m}, 6 \mathrm{H}, 2-\mathrm{H}_{\mathrm{B}} ; 4-\mathrm{H}\right), 1.59-1.44(\mathrm{~m}, 4 \mathrm{H}, 3-\mathrm{H}) .{ }^{13} \mathrm{C}-\mathrm{NMR}\left(101 \mathrm{MHz}, \mathrm{DMSO}-d_{6}\right): \delta(\mathrm{ppm})=54.5(\mathrm{CH}, \mathrm{C} 1)$, $54.3\left(\mathrm{CH}, \mathrm{C} 1{ }^{\prime}\right), 43.9\left(\mathrm{CH}_{2}, \mathrm{C} 5\right), 39.1\left(\mathrm{CH}_{2}, \mathrm{C} 7\right), 27.0\left(\mathrm{CH}_{2}, \mathrm{C} 2\right), 21.7\left(\mathrm{CH}_{2}, \mathrm{C} 4\right), 21.4\left(\mathrm{CH}_{2}, \mathrm{C} 3\right)$. 


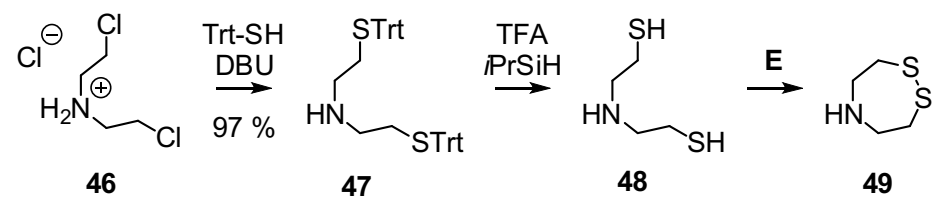

\section{bis((2-(triphenylmethyl)sulfanyl)ethyl))amine (47) ${ }^{29}$}

To bis-(chloroethyl)amine hydrochloride 46 (1.46 g, $8.00 \mathrm{mmol}, 1.0 \mathrm{eq}$.) in anhydrous DMF (24 mL) under nitrogen atmosphere at $0{ }^{\circ} \mathrm{C}$ were added triphenylmethanethiol $(4.79 \mathrm{~g}, 16.80 \mathrm{mmol}, 2.1 \mathrm{eq}$.) and DBU $(4.78 \mathrm{~mL}, 32.0 \mathrm{mmol}$, 4.0 eq.). The cooling bath was removed and the mixture was stirred at room temperature for $3 \mathrm{~h}$, before being concentrated under reduced pressure. The residue was purified by FCC (isohexane/EtOAc) to afford $47(4.82 \mathrm{~g}$, $7.75 \mathrm{mmol}, 97 \%$ ) as a colourless powder. ${ }^{29}$

TLC $\mathrm{R}_{f}=0.42$ (isohexane:EtOAc; 4:1; $1 \% \mathrm{NEt}_{3}$ ). HRMS (El): $\mathrm{C}_{42} \mathrm{H}_{40} \mathrm{NS}_{2}$ [M-e] calc. 622.25967, found 622.25967.

${ }^{1} \mathrm{H}-\mathrm{NMR}\left(500 \mathrm{MHz}, \mathrm{CDCl}_{3}\right): \delta(\mathrm{ppm})=7.36-7.28(\mathrm{~m}, 12 \mathrm{H}), 7.17(\mathrm{dd}, \mathrm{J}=8.6,7.0 \mathrm{~Hz}, 12 \mathrm{H}), 7.12-7.07(\mathrm{~m}, 6 \mathrm{H})$, $2.27(\mathrm{t}, J=6.8 \mathrm{~Hz}, 4 \mathrm{H}), 2.17(\mathrm{t}, J=6.7 \mathrm{~Hz}, 4 \mathrm{H}) .{ }^{13} \mathrm{C}-\mathrm{NMR}\left(126 \mathrm{MHz} \mathrm{CDCl}_{3}\right): \delta(\mathrm{ppm})=144.9\left(C_{\mathrm{Ar}}\right), 129.6\left(C_{\mathrm{Ar}} \mathrm{H}\right)$, $127.9\left(C_{\mathrm{Ar}} \mathrm{H}\right), 126.7\left(C_{\mathrm{Ar}} \mathrm{H}\right), 66.6\left(C_{\text {trityl }}\right), 47.8\left(\mathrm{CH}_{2}\right), 32.2\left(\mathrm{CH}_{2}\right)$.

\section{1,2-dithi-5-azepane (49)}

Step 1: To a $0.16 \mathrm{M}$ solution of 47 (1.0 eq.) in anhydrous $\mathrm{DCM}$ at $0{ }^{\circ} \mathrm{C}$ were added TFA (10 eq.) and triisopropylsilane $\left(2.0\right.$ eq.). The mixture was stirred at $0{ }^{\circ} \mathrm{C}$ for $15 \mathrm{~min}$, the mixture was warmed to r.t., further was stirred for further $4 \mathrm{~h}$ and evaporated under reduced pressure. The residue containing $\mathbf{4 8}$ was directly used in the next step without purification.

Step 2: To a $0.1 \mathrm{M}$ solution in $\mathrm{MeOH}$ of the material obtained in step 1 (assuming quantitative conversion of compound 47) was dropwise added a $0.5 \mathrm{M}$ solution of $\mathrm{I}_{2}$ in $\mathrm{MeOH}$ until a permanently coloured solution was observed (general protocol E). All volatiles were removed and the remaining crude $\mathbf{4 9}$ was directly used without purification.

\section{SS50-type precursor ${ }^{11}$}

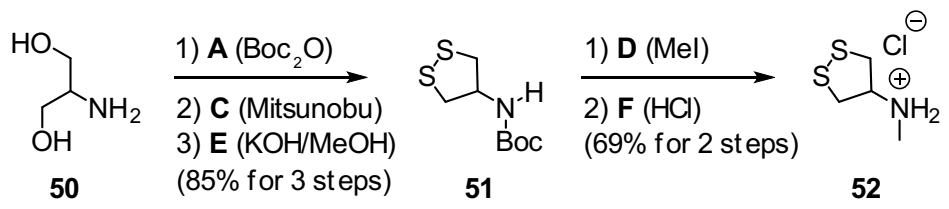

N-Boc 1,2-dithiolan-4-amine (51) 11,30

Step 1: 2-amino-1,3-diol $50(2.50 \mathrm{~g}, 27.4 \mathrm{mmol})$ was treated according to general protocol A to give $\mathrm{N}$-Boc 2-amin-1,3-diol (5.03 g, $26.3 \mathrm{mmol}, 96 \%)$ as a colourless solid and used without further purification.

Step 2: A solution of $\mathrm{N}$-Boc 2-amine-1,3-diol $(4.70 \mathrm{~g}, 24.3 \mathrm{mmol})$ obtained in step 1 was treated according to general protocol $\mathbf{C}$ to give $\mathrm{N}$-Boc 2 -amine-1,3-bis(thioacetate) $(6.63 \mathrm{~g}, 21.6 \mathrm{mmol}, 88 \%)$ as a colourless solid.

Step 3: $N$-Boc 2-amine-1,3-bis(thioacetate) $(4.59 \mathrm{~g}, 14.9 \mathrm{mmol})$ obtained in step 2 was was treated according to general protocol E to afford $51(3.22 \mathrm{~g}, 14.6 \mathrm{mmol}, 98 \%)$ as a yellow solid.

TLC $\mathrm{R}_{f}=0.49$ (isohexane:EtOAc, 10:1). HRMS (ESI ${ }^{-}$): $\mathrm{C}_{8} \mathrm{H}_{14} \mathrm{NO}_{2} \mathrm{~S}_{2}{ }^{-}[\mathrm{M}-\mathrm{H}]^{-}$: calc. 220.04714, found 220.04718. ${ }^{1} \mathrm{H}-\mathrm{NMR}\left(400 \mathrm{MHz}, \mathrm{CDCl}_{3}\right): \delta(\mathrm{ppm})=5.06(\mathrm{~d}, J=3.9 \mathrm{~Hz}, 1 \mathrm{H}, \mathrm{NH}), 4.95(\mathrm{~s}, 1 \mathrm{H}, \mathrm{CH}), 3.21(\mathrm{dd}, J=11.6,4.8 \mathrm{~Hz}$, $2 \mathrm{H}), 3.09$ (dd, $J=11.6,2.0 \mathrm{~Hz}, 2 \mathrm{H}), 1.44(\mathrm{~s}, 9 \mathrm{H})$.

\section{$\mathrm{N}$-methyl-1,2-dithiolan-4-amine hydrochloride (52) ${ }^{11}$}

Step 1: $5(2.00 \mathrm{~g}, 9.04 \mathrm{mmol})$ was treated according to general protocol D to give $N$-Boc $N$-methyl 1,2-dithiolane4-amine as a yellow oil.

Step 2: $N$-Boc $N$-methyl 1,2-dithiolane-4-amine (118 mg, $0.501 \mathrm{mmol}$ ) obtained in step 1 was treated according to general protocol $\mathbf{F}$ to give hydrochloride $\mathbf{5 2}(85 \mathrm{mg}, 0.50 \mathrm{mmol}$, quant.) as a colourless solid.

${ }^{1} \mathrm{H}-\mathrm{NMR}\left(400 \mathrm{MHz}, \mathrm{DMSO}-\mathrm{d}_{6}\right): \delta(\mathrm{ppm})=8.49(\mathrm{~s}, 2 \mathrm{H}), 3.35(\mathrm{p}, J=5.1 \mathrm{~Hz}, 1 \mathrm{H}), 2.58(\mathrm{dd}, J=5.2,1.7 \mathrm{~Hz}, 4 \mathrm{H}), 1.74$ $(\mathrm{s}, 3 \mathrm{H}) .{ }^{13} \mathrm{C}$ NMR $\left(101 \mathrm{MHz}, \mathrm{DMSO}-d_{6}\right): \delta(\mathrm{ppm})=63.3(\mathrm{CH}), 40.4\left(\mathrm{CH}_{2}\right), 31.1\left(\mathrm{CH}_{3}\right)$. 


\subsection{Cargo synthesis}

\section{6-chloro-2-(5-chloro-2-hydroxyphenyl)quinazolin-4(3H)-one (PQ-OH, 55) ${ }^{31}$}<smiles>O=Cc1cc(Cl)ccc1O</smiles>

53<smiles>NC(=O)c1cc(Cl)ccc1N</smiles>

54<smiles>O=c1[nH]c(-c2cc(Cl)ccc2O)nc2ccc(Cl)cc12</smiles>

55

A mixture of 5-chloro-salicylaldehyde $53(1.92 \mathrm{~g}, 12.3 \mathrm{mmol}), 2$-amino-5-chlorobenzamide 54 (2.15 g, $12.6 \mathrm{mmol})$, $\mathrm{TsOH}(0.23 \mathrm{~g}, 1.2 \mathrm{mmol})$ and anhydrous $\mathrm{EtOH}(250 \mathrm{~mL})$ was heated to reflux for $1 \mathrm{~h}$. Then, the mixture was cooled to $0{ }^{\circ} \mathrm{C}$, DDQ $(2.72 \mathrm{~g}, 12.0 \mathrm{mmol})$ was added portionwise and stirring was continued at $0{ }^{\circ} \mathrm{C}$ for $1 \mathrm{~h}$. The resulting precipitate was collected, washed with cold, anhydrous EtOH and dried under reduced pressure to afford $\mathbf{P Q}-\mathbf{O H}$ (55) (3.32 g, $10.5 \mathrm{mmol}, 88 \%)$ as a faint yellow solid.

Bright green fluorescence was observed, when the solid PQ-OH (55) was exposed to UV light. ${ }^{31}$

HRMS (El): $\mathrm{C}_{14} \mathrm{H}_{8} \mathrm{Cl}_{2} \mathrm{~N}_{2} \mathrm{O}_{2}$ [M-e] calc. 305.9958, found 305.9955. ${ }^{1} \mathrm{H}-\mathrm{NMR}$ (400 MHz, DMSO-d $): \delta(p p m)=13.36$ $(\mathrm{s}, 1 \mathrm{H}), 12.62(\mathrm{~s}, 1 \mathrm{H}), 8.29(\mathrm{~d}, J=2.6 \mathrm{~Hz}, 1 \mathrm{H}), 8.10(\mathrm{~d}, J=2.3 \mathrm{~Hz}, 1 \mathrm{H}), 7.90(\mathrm{dd}, J=8.7,2.4 \mathrm{~Hz}, 1 \mathrm{H}), 7.85(\mathrm{~d}$, $J=8.8 \mathrm{~Hz}, 1 \mathrm{H}), 7.50(\mathrm{dd}, J=8.9,2.6 \mathrm{~Hz}, 1 \mathrm{H}), 7.05(\mathrm{~d}, J=8.9 \mathrm{~Hz}, 1 \mathrm{H})$.

\section{3'-hydroxy-6'-methoxy-3H-spiro[isobenzofuran-1,9'-xanthen]-3-one (MF-OH, 58)}

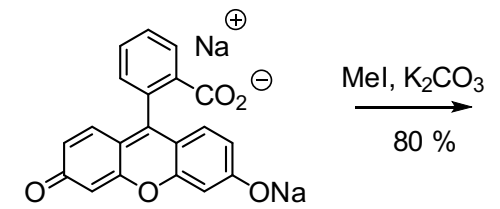

56<smiles>COc1ccc2c(-c3ccccc3OC)c3ccc(=O)cc-3oc2c1</smiles>

57

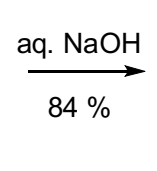<smiles>COc1ccc2c(c1)Oc1cc(O)ccc1C21OC(=O)c2ccccc21</smiles>

58

Step 1: To disodium fluorescein $56(10.0 \mathrm{~g}, 26.6 \mathrm{mmol})$ in anhydrous DMF $(90 \mathrm{~mL})$ was portionwise added $\mathrm{K}_{2} \mathrm{CO}_{3}$ $(9.2 \mathrm{~g}, 66.4 \mathrm{mmol})$ and the mixture was stirred for $1 \mathrm{~h}$. lodomethane $(4.96 \mathrm{~mL}, 79.7 \mathrm{mmol})$ was added dropwise and the resulting mixture was further stirred at r.t. for $12 \mathrm{~h}$, before ice water $(100 \mathrm{~mL})$ was added carefully. After stirring at $0{ }^{\circ} \mathrm{C}$ for $30 \mathrm{~min}$, the resulting yellow precipitate was collected, washed with water and dried to obtain $\mathbf{5 7}$ $(7.68 \mathrm{~g}, 21.3 \mathrm{mmol}, 80 \%)$, which was used without further purification.

Step 2: To the material obtained in step 1 ( $7.68 \mathrm{~g}, 21.3 \mathrm{mmol})$ in $\mathrm{MeOH}(150 \mathrm{~mL})$ was dropwise added $2 \mathrm{M}$ aq. $\mathrm{NaOH}(50 \mathrm{~mL})$ and the resulting mixture was stirred for $10 \mathrm{~h}$, before the $\mathrm{MeOH}$ was evaporated under reduced pressure. The residue was washed with $\mathrm{Et}_{2} \mathrm{O}(3 \times 100 \mathrm{~mL})$, before being acidified with $6 \mathrm{M}$ aq. $\mathrm{HCl}(18 \mathrm{~mL})$. The resulting precipitate was collected, washed with water $(3 \times 100 \mathrm{~mL})$ and dried under reduced pressure to afford MF-OH (58) $(6.2 \mathrm{~g}, 17.9 \mathrm{mmol}, 84 \%)$ as a yellow solid.

Bright green fluorescence was observed, when an aqueous solution of MF-OH (58) was exposed to UV light. ${ }^{32}$

TLC $\mathrm{R}_{f}=0.42$ (isohexane:EtOAc; 1:1). HRMS $\left(E S I^{+}\right): \mathrm{C}_{21} \mathrm{H}_{15} \mathrm{O}_{5}{ }^{+}[\mathrm{M}+\mathrm{H}]^{+}$: calc. 347.09140, found 347.09155.

${ }^{1} \mathrm{H}-N M R\left(500 \mathrm{MHz}, \mathrm{DMSO}-\mathrm{d}_{6}\right): \delta(\mathrm{ppm})=10.16(\mathrm{~s}, 1 \mathrm{H}), 8.00(\mathrm{~d}, J=7.6 \mathrm{~Hz}, 1 \mathrm{H}), 7.79(\mathrm{td}, J=7.5,1.1 \mathrm{~Hz}, 1 \mathrm{H})$, $7.72(\mathrm{td}, J=7.5,0.8 \mathrm{~Hz}, 1 \mathrm{H}), 7.27(\mathrm{~d}, J=7.7 \mathrm{~Hz}, 1 \mathrm{H}), 6.93(\mathrm{~d}, J=2.5 \mathrm{~Hz}, 1 \mathrm{H}), 6.72-6.70(\mathrm{~m}, 1 \mathrm{H}), 6.69(\mathrm{~d}$, $J=2.5 \mathrm{~Hz}, 1 \mathrm{H}), 6.64(\mathrm{~d}, J=8.8 \mathrm{~Hz}, 1 \mathrm{H}), 6.57(\mathrm{~d}, J=1.0 \mathrm{~Hz}, 2 \mathrm{H}), 3.81(\mathrm{~s}, 3 \mathrm{H})$.

\section{2',7'-difluoro-3'-hydroxy-6'--isopropoxy-3H-spiro[isobenzofuran-1,9'-xanthen]-3-one (IG-OH, 62)}<smiles>Oc1ccc(F)c(O)c1</smiles>

59<smiles>O=C1OC(=O)C2C=CC=C12</smiles>

60

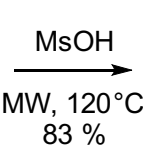
$83 \%$<smiles>O=C1Oc2ccc(O)cc2C(c2ccccc2)(c2ccc(F)c(O)c2)O1</smiles>

61

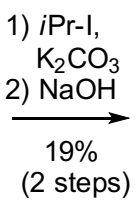

(2 steps)<smiles>CC(C)Oc1cc2c(cc1F)C1(OC(=O)c3ccccc31)c1cc(F)c(O)cc1O2</smiles>

62

Step 1: 4-fluororesorcinol $59(1.00 \mathrm{~g}, 7.80 \mathrm{mmol})$ and phthalic anhydride 60 (0.59 g, $4.0 \mathrm{mmol})$ were dissolved in $\mathrm{MsOH}(5 \mathrm{~mL})$ and heated to $120^{\circ} \mathrm{C}$ by microwave irradiation for $5 \mathrm{~min}$. The mixture cooled to r.t., poured into icecold water $(150 \mathrm{~mL})$ and the resulting suspension was stirred at $r . t$. for $1 \mathrm{~h}$. The precipitate was collected, washed with water $(50 \mathrm{~mL})$ and dried under reduced pressure to afford crude $61(1.19 \mathrm{~g}, 3.23 \mathrm{mmol}, 83 \%)$ as a yellow solid, which was used without further purification.

Step 2: To a suspension of the material obtained in step 1 (1.19 g, $3.23 \mathrm{mmol}, 1.0$ eq.) and $\mathrm{K}_{2} \mathrm{CO}_{3}(1.33 \mathrm{~g}$, $9.62 \mathrm{mmol}, 3.0 \mathrm{eq})$ in anhydrous DMF $(25 \mathrm{~mL})$ under nitrogen atmosphere was added isopropyliodide $(3.28 \mathrm{~mL}$, 
$32.1 \mathrm{mmol}$ and the mixture was stirred for $15 \mathrm{~h}$, before all volatiles were removed under reduced pressure. The residue was directly used in the next step without purification.

Step 3: To the material obtained in step 2 in $\mathrm{MeOH}(20 \mathrm{~mL})$ was added $2 \mathrm{M}$ aq. $\mathrm{NaOH}(1.6 \mathrm{~mL})$ and the mixture was stirred for $15 \mathrm{~h}$ before the $\mathrm{MeOH}$ was evaporated under reduced pressure. $\mathrm{Et}_{2} \mathrm{O}(50 \mathrm{~mL})$ and diluted aq. $\mathrm{HCl}$ $\left(100^{\circ} \mathrm{mL}, \mathrm{pH} 2\right)$ were added. The organic layer was separated and the aq. layer was extracted with $\mathrm{Et}_{2} \mathrm{O}(3 \times 50 \mathrm{~mL})$. The combined organic layers were dried over $\mathrm{Na}_{2} \mathrm{SO}_{4}$, filtered and evaporated under reduced pressure. The residue was purified by FCC (isohexane/EtOAc) to afford IG-OH (62) $(0.25 \mathrm{~g}, 0.61 \mathrm{mmol}, 19 \%)$ as an orange solid.

Bright green fluorescence was observed, when an aqueous solution of IG-OH (62) was exposed to UV light.<smiles>CC(C)Oc1cc2c(cc1F)C1(OC(=O)c3ccccc31)c1cc(F)c(O)cc1O2</smiles>

TLC $\mathrm{R}_{f}=0.47$ (isohexane:EtOAc; 1:1). HRMS $\left(E S{ }^{+}\right): \mathrm{C}_{23} \mathrm{H}_{17} \mathrm{~F}_{2} \mathrm{O}_{5}{ }^{+}[\mathrm{M}+\mathrm{H}]^{+}$: calc. 411.10386, found 411.10356.

${ }^{1} \mathrm{H}-\mathrm{NMR}\left(400 \mathrm{MHz}, \mathrm{DMSO}-\mathrm{d}_{6}\right): \delta(\mathrm{ppm})=10.77(\mathrm{~s}, 1 \mathrm{H}, 22-\mathrm{H}), 8.00(\mathrm{~d}, J=7.5 \mathrm{~Hz}, 1 \mathrm{H}, 17-\mathrm{H}), 7.80(\mathrm{t}, \mathrm{J}=7.5 \mathrm{~Hz}$, $1 \mathrm{H}, 15-\mathrm{H}), 7.74(\mathrm{t}, J=7.4 \mathrm{~Hz}, 1 \mathrm{H}, 16-\mathrm{H}), 7.30(\mathrm{~d}, J=7.6 \mathrm{~Hz}, 1 \mathrm{H}, 14-\mathrm{H}), 7.20(\mathrm{~d}, J=7.3 \mathrm{~Hz}, 1 \mathrm{H}, 6-\mathrm{H}), 6.90(\mathrm{~d}$, $J=7.5 \mathrm{~Hz}, 1 \mathrm{H}, 3-\mathrm{H}), 6.55(\mathrm{~d}, J=11.5 \mathrm{~Hz}, 1 \mathrm{H}, 11-\mathrm{H}), 6.51(\mathrm{~d}, J=11.3 \mathrm{~Hz}, 1 \mathrm{H}, 8-\mathrm{H}), 4.79(\mathrm{p}, J=6.0 \mathrm{~Hz}, 1 \mathrm{H}, 20-\mathrm{H})$, $1.32(\mathrm{dd}, J=5.8,4.1 \mathrm{~Hz}, 6 \mathrm{H}, 21-\mathrm{H})$.

${ }^{13} \mathrm{C}-N M R\left(101 \mathrm{MHz}, \mathrm{CDCl}_{3}\right): \delta(\mathrm{ppm})=169.2(C=\mathrm{O}, \mathrm{C} 19), 152.1,151.0,149.0,148.6,148.5,148.5,148.4,148.1$ $\left(\mathrm{d}, J=2.0 \mathrm{~Hz}, C_{\mathrm{Ar}}, \mathrm{C} 2\right), 146.7,135.5\left(C_{\mathrm{Ar}} \mathrm{H}, \mathrm{C} 15\right), 130.4\left(C_{\mathrm{Ar}} \mathrm{H}, \mathrm{C} 16\right), 126.7\left(C_{\mathrm{Ar}} \mathrm{H}, \mathrm{C} 17\right), 125.6,124.1\left(C_{\mathrm{Ar}} \mathrm{H}, \mathrm{C} 14\right)$ $114.1\left(\mathrm{~d}, J=21.4 \mathrm{~Hz}, C_{\mathrm{Ar}} \mathrm{H}, \mathrm{C} 3\right), 113.7\left(\mathrm{~d}, J=20.8 \mathrm{~Hz}, C_{\mathrm{Ar}} \mathrm{H}, \mathrm{C} 8\right), 110.4\left(\mathrm{~d}, J=6.0 \mathrm{~Hz}, C_{\mathrm{Ar}}, \mathrm{C} 2\right), 109.5(\mathrm{~d}$, $\left.J=6.1 \mathrm{~Hz}, C_{\mathrm{Ar}}, \mathrm{C} 7\right), 105.4\left(C_{\mathrm{Ar}} \mathrm{H}, \mathrm{C} 6\right), 104.1\left(\mathrm{C}_{\mathrm{Ar}} \mathrm{H}, \mathrm{C} 11\right), 72.5\left(C_{\text {spiro }}, \mathrm{C} 12\right), 29.8(\mathrm{CH}, \mathrm{C} 20), 22.0\left(\mathrm{CH}_{3}, \mathrm{C} 21\right)$.

\section{3'-amino-6'-methoxy-3H-spiro[isobenzofuran-1,9'-xanthen]-3-one ( $\mathrm{MR}^{\left.-\mathrm{NH}_{2}, 64\right)}$}<smiles>COc1ccc2c(c1)Oc1cc(O)ccc1C21OC(=O)c2ccccc21</smiles>

58

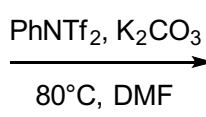

$80^{\circ} \mathrm{C}, \mathrm{DMF}$<smiles>COc1ccc2c(c1)Oc1cc(O)ccc1C21OC(=O)c2ccccc21</smiles>

63

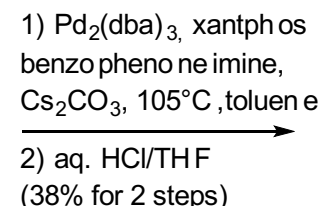

$(38 \%$ for 2 steps $)$

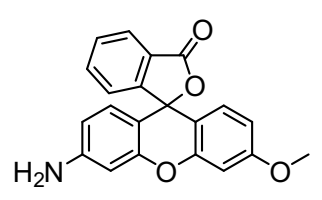

64

Step 1: A mixture of 58 (800 mg, $2.31 \mathrm{mmol}, 1.0$ eq.), $\mathrm{K}_{2} \mathrm{CO}_{3}$ (479 mg, $3.46 \mathrm{mmol}, 1.5$ eq.), bis(trifluoromethane sulfonyl)aniline (908 mg, $2.54 \mathrm{mmol}, 1.1 \mathrm{eq}$.) and anhydrous DMF (10 mL) was stirred at $80{ }^{\circ} \mathrm{C}$ for $1.5 \mathrm{~h}$. Then, the solvent was evaporated under reduced pressure. The residue was taken up in DCM $(10 \mathrm{~mL})$, the mixture was filtered over silica $(6 \mathrm{~g})$ and the silica was washed with additional DCM $(60 \mathrm{~mL})$. The solvent was removed under reduced pressure and the residue containing 63 was directly used for further steps without purification.

Step 2: To the material obtained in step 1 were added $\mathrm{Pd}_{2}(\mathrm{dba})_{3}(212 \mathrm{mg}, 0.231 \mathrm{mmol}, 0.1$ eq. $)$, Xantphos (200 mg, $0.346 \mathrm{mmol}, 0.15$ eq.), $\mathrm{Cs}_{2} \mathrm{CO}_{3}(1.05 \mathrm{~g}, 3.23 \mathrm{mmol}, 1.4$ eq.) and toluene $(25 \mathrm{~mL})$. The mixture was degassed by evacuating under sonication and refilling with nitrogen, before benzophenone imine $(465 \mu \mathrm{L}$, $2.77 \mathrm{mmol}, 1.2$ eq.) was added. Then, the mixture was again degassed and warmed to $105^{\circ} \mathrm{C}$. After stirring at $105^{\circ} \mathrm{C}$ for $15 \mathrm{~h}$, the mixture was cooled to r.t. and filtered over Celite ${ }^{\circledR}$. The Celite ${ }^{\circledR}$ was washed with DCM until the filtrate turned colourless. The filtrate was evaporated under reduced pressure, then THF $(30 \mathrm{~mL})$ and $1 \mathrm{M} \mathrm{aq.} \mathrm{HCl}$ $(3 \mathrm{~mL})$ were added. After stirring for $1 \mathrm{~h}$, water $(40 \mathrm{~mL})$ was added and the mixture was extracted with EtOAc $(3 \times 40 \mathrm{~mL})$. The extracts were washed with sat. aq. $\mathrm{NaCl}(40 \mathrm{~mL})$, dried over $\mathrm{Na}_{2} \mathrm{SO}_{4}$, filtered and evaporated under reduced pressure. The residue was purified by FCC (0 to $30 \%$ EtOAc in DCM) to afford compound MR-OH (64) (302 mg, $0.874 \mathrm{mmol}, 38 \%$ ) as an orange solid.

Bright green fluorescence was observed, when an aqueous solution of $\mathbf{M R}-\mathrm{NH}_{2}(\mathbf{6 4})$ was exposed to UV light.

TLC $R_{f}=0.43(30 \%$ EtOAc in DCM).

${ }^{1} \mathrm{H}-N M R\left(400 \mathrm{MHz}, \mathrm{DMSO}-\mathrm{d}_{6}\right): \delta(\mathrm{ppm})=7.97(\mathrm{dd}, J=7.6,1.2 \mathrm{~Hz}, 1 \mathrm{H}), 7.78(\mathrm{td}, J=7.5,1.2 \mathrm{~Hz}, 1 \mathrm{H}), 7.70(\mathrm{td}, J=$ $7.4,1.0 \mathrm{~Hz}, 1 \mathrm{H}), 7.25(\mathrm{~d}, J=7.7 \mathrm{~Hz}, 1 \mathrm{H}), 6.90(\mathrm{~d}, J=2.5 \mathrm{~Hz}, 1 \mathrm{H}), 6.67(\mathrm{dd}, J=8.8,2.5 \mathrm{~Hz}, 1 \mathrm{H}), 6.59(\mathrm{~d}, J=8.8 \mathrm{~Hz}$, $1 \mathrm{H}), 6.43(\mathrm{~d}, J=2.1 \mathrm{~Hz}, 1 \mathrm{H}), 6.37(\mathrm{~d}, J=8.5 \mathrm{~Hz}, 1 \mathrm{H}), 6.34(\mathrm{~s}, 1 \mathrm{H}), 5.68(\mathrm{~s}, 2 \mathrm{H}), 3.80(\mathrm{~s}, 3 \mathrm{H})$.

The spectroscopic data agrees with previously reported values. ${ }^{33}$ 


\subsection{Probe synthesis}

\section{Bz-Series}

trans-(hexahydro-[1,2]dithiino[4,5-b]pyridin-1(2H)-yl)benzamide (SS66T-Bz, 65)
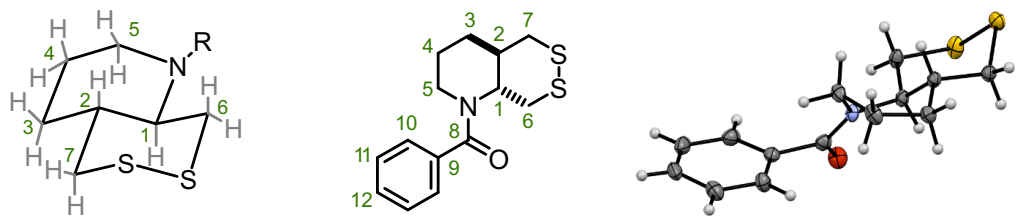

Prepared from $8(16.7 \mathrm{mg}, 0.079 \mathrm{mmol})$ and $\mathrm{BzCl}(15.0 \mu \mathrm{L}, 0.128 \mathrm{mmol})$ according to general protocol $\mathbf{H}$. Purification by FCC (isohexane/EtOAc) gave $65(14.5 \mathrm{mg}, 0.052 \mathrm{mmol}, 66 \%)$ as a colourless solid. Unambiguous confirmation of the absolute configuration was derived from a single-crystal X-Ray analysis.

TLC $\mathrm{R}_{f}=0.59$ (isohexane:EtOAc, 2:1). HRMS (EI): $\mathrm{C}_{14} \mathrm{H}_{17} \mathrm{NOS}_{2}$ [M-e] calc. 279.0747, found 279.0747.

${ }^{1} \mathrm{H}-\mathrm{NMR}\left(500 \mathrm{MHz}, \mathrm{CDCl}_{3}\right): \delta(\mathrm{ppm})=7.42-7.38(\mathrm{~m}, 3 \mathrm{H}, 10-\mathrm{H} ; 12-\mathrm{H}), 7.38-7.34(\mathrm{~m}, 2 \mathrm{H}, 11-\mathrm{H}), 4.13\left(\mathrm{td},{ }^{3} J_{1-6}=\right.$ $\left.10.7 \mathrm{~Hz},{ }^{3} J_{1-6^{\prime}}=2.9 \mathrm{~Hz}, 1 \mathrm{H}, 1-\mathrm{H}\right), 3.48\left(\mathrm{ddd},{ }^{2} J_{5^{\prime}-5}=14.2 \mathrm{~Hz},{ }^{3} J_{5^{\prime}-4}=7.3 \mathrm{~Hz},{ }^{3} J_{5^{\prime}-4^{\prime}}=2.0 \mathrm{~Hz}, 1 \mathrm{H}, 5^{\prime}-\mathrm{H}\right), 3.32$ (ddd, $\left.{ }^{2} J_{5-5^{\prime}}=14.1 \mathrm{~Hz},{ }^{3} J_{5-4}=11.2 \mathrm{~Hz},{ }^{3} J_{5-4^{\prime}}=5.4 \mathrm{~Hz}, 1 \mathrm{H}, 5-\mathrm{H}\right), 3.22\left(\mathrm{dd},{ }^{2} J_{6^{\prime}-6}=12.7 \mathrm{~Hz},{ }^{3} J_{6^{\prime}-1}=2.9 \mathrm{~Hz}, 1 \mathrm{H}, 6^{\prime}-\mathrm{H}\right), 3.08$ $\left(\mathrm{dd},{ }^{2} \mathrm{~J}_{6-6^{\prime}}=12.7 \mathrm{~Hz},{ }^{3} \mathrm{~J}_{6-1}=10.4 \mathrm{~Hz}, 1 \mathrm{H}, 6-\mathrm{H}\right), 2.96\left(\mathrm{dd},{ }^{2} \mathrm{~J}_{7-7}=13.4 \mathrm{~Hz},{ }^{3} \mathrm{~J}_{7-2}=10.9 \mathrm{~Hz}, 1 \mathrm{H}, 7-\mathrm{H}\right), 2.80\left(\mathrm{dd},{ }^{2} \mathrm{~J}_{7^{\prime}-7}=\right.$ $\left.13.5 \mathrm{~Hz},{ }^{3} \mathrm{~J}^{\prime}-2=2.6 \mathrm{~Hz}, 1 \mathrm{H}, 7^{\prime}-\mathrm{H}\right), 2.20\left(\mathrm{dtt}, J=13.5 \mathrm{~Hz},{ }^{3} J_{2-7}=11.0 \mathrm{~Hz},{ }^{3} J_{2-7}=2.7 \mathrm{~Hz}, 1 \mathrm{H}, 2-\mathrm{H}\right), 1.83-1.72(\mathrm{~m}$, $1 \mathrm{H}, 4-\mathrm{H}), 1.70-1.59(\mathrm{~m}, 2 \mathrm{H}, 4-\mathrm{H} ; 3-\mathrm{H}), 1.46-1.34(\mathrm{~m}, 1 \mathrm{H}, 3-\mathrm{H})$.

${ }^{13} \mathrm{C}$-NMR $\left(126 \mathrm{MHz}, \mathrm{CDCl}_{3}\right): \delta(\mathrm{ppm})=171.6(\mathrm{C}=\mathrm{O}, \mathrm{C} 8), 136.8\left(C_{\mathrm{Ar}}, \mathrm{C} 9\right), 129.8\left(C_{\mathrm{Ar}} \mathrm{H}, \mathrm{C} 10\right), 128.7\left(C_{\mathrm{Ar}} \mathrm{H}, \mathrm{C} 12\right)$, $126.8\left(\mathrm{C}_{\mathrm{Ar}} \mathrm{H}, \mathrm{C} 11\right), 61.6(\mathrm{CH}, \mathrm{C} 1), 42.0\left(\mathrm{CH}_{2}, \mathrm{C} 5\right), 41.1\left(\mathrm{CH}_{2}, \mathrm{C} 7\right), 40.5(\mathrm{CH}, \mathrm{C} 2), 36.3\left(\mathrm{CH}_{2}, \mathrm{C} 6\right), 26.7\left(\mathrm{CH}_{2}, \mathrm{C} 3\right)$, $23.8\left(\mathrm{CH}_{2}, \mathrm{C} 4\right)$.

cis-(hexahydro-[1,2]dithiino[4,5-b]pyridin-1(2H)-yI)benzamide (SS66C-Bz, 66)
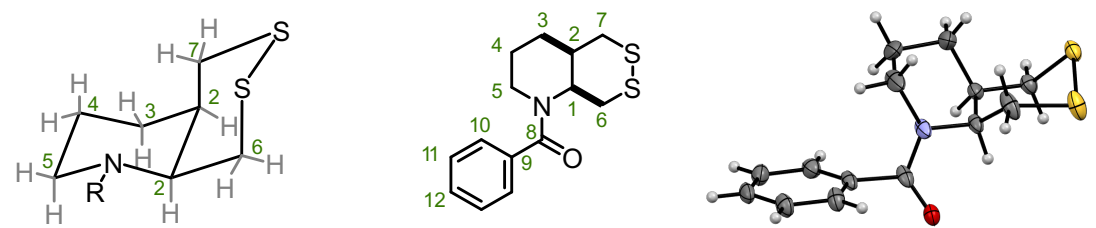

Prepared from crude 18 (corresponding to $20.0 \mathrm{mg}, 0.068 \mathrm{mmol}$ of compound 12) and $\mathrm{BzCl}(16 \mu \mathrm{L}, 0.135 \mathrm{mmol}$ ) according to general protocol $\mathrm{H}$. Compound $66(7.8 \mathrm{mg}, 0.028 \mathrm{mmol}, 41 \%)$ was obtained as a colourless solid after FCC (isohexane/EtOAc). Unambiguous confirmation of the absolute configuration was derived from a singlecrystal X-Ray analysis.

TLC $\mathrm{R}_{f}=0.48$ (isohexane:EtOAc, 2:1). HRMS (El): $\mathrm{C}_{14} \mathrm{H}_{17} \mathrm{NOS}_{2}$ [M-e] calc. 279.0747, found 279.0744.

Individual rotamers were observed by NMR spectroscopy at $298 \mathrm{~K}$ (ratio ca. 2:1) and measurements at $373 \mathrm{~K}$ were not sufficient to obtain time-averaged spectra. The following data refers to the major rotamer and is supported by 2D-NMR and TOCSY experiments.

${ }^{1} \mathrm{H}-N M R\left(400 \mathrm{MHz}, \mathrm{CDCl}_{3}\right): \delta(\mathrm{ppm})=7.52-7.30(\mathrm{~m}, 5 \mathrm{H}, 10-\mathrm{H} ; 11-\mathrm{H} ; 12-\mathrm{H}), 5.07(\mathrm{~d}, \mathrm{~J}=12.0 \mathrm{~Hz}, 1 \mathrm{H}, 1-\mathrm{H}), 3.70$ - $3.44\left(\mathrm{~m}, 3 \mathrm{H}, 5-\mathrm{H}_{\mathrm{A}} ; 6-\mathrm{H}_{\mathrm{A}} ; 7-\mathrm{H}_{\mathrm{A}}\right), 3.03\left(\mathrm{t}, J=13.3 \mathrm{~Hz}, 1 \mathrm{H}, 5-\mathrm{H}_{\mathrm{B}}\right), 2.81\left(\mathrm{~d}, J=14.1 \mathrm{~Hz}, 1 \mathrm{H}, 7-\mathrm{H}_{\mathrm{B}}\right), 2.56(\mathrm{~d}$, $\left.J=12.9 \mathrm{~Hz}, 1 \mathrm{H}, 6-\mathrm{H}_{\mathrm{B}}\right), 2.37\left(\mathrm{t}, J=13.4 \mathrm{~Hz}, 1 \mathrm{H}, 4-\mathrm{H}_{\mathrm{A}}\right), 2.27(\mathrm{~d}, J=13.7 \mathrm{~Hz}, 1 \mathrm{H}, 2-\mathrm{H}), 1.80-1.68\left(\mathrm{~m}, 1 \mathrm{H}, 3-\mathrm{H}_{\mathrm{A}}\right)$, $1.64\left(\mathrm{br}-\mathrm{s}, 1 \mathrm{H}, 3-\mathrm{H}_{\mathrm{B}}\right), 1.53\left(\mathrm{~d}, \mathrm{~J}=13.7 \mathrm{~Hz}, 1 \mathrm{H}, 4-\mathrm{H}_{\mathrm{B}}\right)$.

${ }^{13} \mathrm{C}-N M R\left(101 \mathrm{MHz}, \mathrm{CDCl}_{3}\right): \delta(\mathrm{ppm})=170.8(\mathrm{C}=\mathrm{O}, \mathrm{C} 8), 136.2\left(C_{\mathrm{Ar}}, \mathrm{C} 9\right), 129.8\left(C_{\mathrm{Ar}} \mathrm{H}, \mathrm{C} 12\right), 128.7\left(C_{\mathrm{Ar}} \mathrm{H}, \mathrm{C} 10\right)$, $126.9\left(\mathrm{C}_{\mathrm{Ar}} \mathrm{H}, \mathrm{C} 11\right), 50.8(\mathrm{CH}, \mathrm{C} 1), 43.8\left(\mathrm{CH}_{2}, \mathrm{C} 7\right), 42.1\left(\mathrm{CH}_{2}, \mathrm{C} 5\right), 35.8(\mathrm{CH}, \mathrm{C} 2), 29.5\left(\mathrm{CH}_{2}, \mathrm{C} 6\right), 26.1\left(\mathrm{CH}_{2}, \mathrm{C} 3\right)$, $23.9\left(\mathrm{CH}_{2}, \mathrm{C} 4\right)$. 
(S)-N-methyl-N-(1,2-dithian-4-yl)benzamide (SS60-Bz, 67)
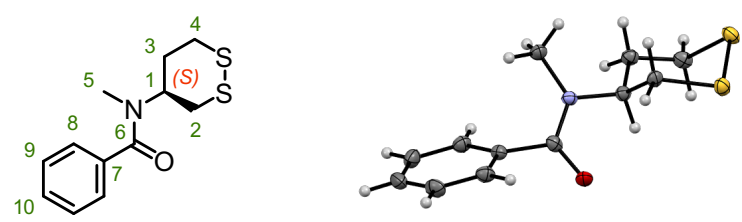

Prepared from $25(74 \mathrm{mg}, 0.40 \mathrm{mmol})$ and $\mathrm{BzCl}(2.7$ eq.) according to general protocol H. Purification by FCC (isohexane/EtOAc) gave 67 ( $80 \mathrm{mg}, 0.31 \mathrm{mmol}, 79 \%$ ) a colourless solid. Unambiguous confirmation of the absolute configuration was derived from a single-crystal X-Ray analysis.

TLC $R_{f}=0.28$ (isohexane:EtOAc, 2:1). HRMS (EI): $\mathrm{C}_{12} \mathrm{H}_{15} \mathrm{NOS}_{2}$ [M-e] calc. 253.0590, found 253.0587.

${ }^{1} \mathrm{H}-\mathrm{NMR}\left(400 \mathrm{MHz}\right.$, DMSO-d $\mathrm{d}_{6}, 373 \mathrm{~K}$ ): $\delta(\mathrm{ppm})=7.48-7.41(\mathrm{~m}, 3 \mathrm{H}, 8-\mathrm{H}$; 10-H), $7.39-7.33(\mathrm{~m}, 2 \mathrm{H}, 9-\mathrm{H}), 4.19$ (s, $1 \mathrm{H}, 1-\mathrm{H}), 3.19\left(\mathrm{dd}, J=12.9,11.2 \mathrm{~Hz}, 1 \mathrm{H}, 2-\mathrm{H}_{\mathrm{A}}\right), 3.11\left(\mathrm{dt}, J=13.7,4.0 \mathrm{~Hz}, 1 \mathrm{H}, 3-\mathrm{H}_{\mathrm{A}}\right), 3.02(\mathrm{~d}, J=11.0 \mathrm{~Hz}, 1 \mathrm{H}$, $\left.2-\mathrm{H}_{\mathrm{B}}\right), 2.91-2.82\left(\mathrm{~m}, 1 \mathrm{H}, 3-\mathrm{H}_{\mathrm{B}}\right), 2.84(\mathrm{~s}, 3 \mathrm{H}, 5-\mathrm{H}), 2.21-2.04(\mathrm{~m}, 2 \mathrm{H}, 4-\mathrm{H})$.

${ }^{13} \mathrm{C}-\mathrm{NMR}\left(101 \mathrm{MHz}\right.$, DMSO- $\left.d_{6}, 373 \mathrm{~K}\right): \delta(\mathrm{ppm})=169.7(\mathrm{C}=\mathrm{O}, 6-\mathrm{H}), 136.4\left(\mathrm{C}_{\mathrm{Ar}}, 7-\mathrm{H}\right), 128.6\left(\mathrm{C}_{\mathrm{Ar}} \mathrm{H}, 8-\mathrm{H}\right), 127.8$ $\left(\mathrm{C}_{\mathrm{Ar}} \mathrm{H}, 9-\mathrm{H}\right), 125.9\left(\mathrm{C}_{\mathrm{Ar}} \mathrm{H}, 10-\mathrm{H}\right), 54.9(\mathrm{CH}, 1-\mathrm{H}), 35.1\left(\mathrm{CH}_{2}, 2-\mathrm{H}\right), 34.7\left(\mathrm{CH}_{2}, 3-\mathrm{H}\right), 31.5\left(\mathrm{CH}_{2}, 4-\mathrm{H}\right), 30.0\left(\mathrm{CH}_{3}, 5-\mathrm{H}\right)$.

$\mathbf{N}$-methyl-N-(1,2-dithiolan-4-yl)benzamide (SS50-Bz, 68)

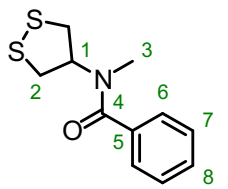

Prepared from $52(43.0 \mathrm{mg}, 0.250 \mathrm{mmol})$ and $\mathrm{BzCl}(4.0$ eq.) according to general protocol H. Purification by FCC (isohexane/EtOAc) gave $67(21.7 \mathrm{mg}, 0.091 \mathrm{mmol}, 36 \%)$ as a colourless solid.

TLC $R_{f}=0.44$ (isohexane:EtOAc, 2:1). HRMS (EI): for $\mathrm{C}_{11} \mathrm{H}_{13} \mathrm{NOS}_{2}$ [M-e] calc. 239.0434, found 239.0432.

Individual rotamers were observed by NMR spectroscopy at $298 \mathrm{~K}$ (ratio ca. 1:1) and time-averaged spectra were obtained at $373 \mathrm{~K}$.

${ }^{1} \mathrm{H}-\mathrm{NMR}\left(400 \mathrm{MHz}\right.$, DMSO- $\left.\mathrm{d}_{6}, 373 \mathrm{~K}\right): \delta(\mathrm{ppm})=7.50-7.43(\mathrm{~m}, 3 \mathrm{H}, 6-\mathrm{H} ; \mathrm{8}-\mathrm{H}), 7.43-7.37(\mathrm{~m}, 2 \mathrm{H}, 7-\mathrm{H}), 5.03$ (s, $1 \mathrm{H}, 1-\mathrm{H}), 3.37\left(\mathrm{dd},{ }^{2} J_{2 \mathrm{~A}-2 \mathrm{~B}}=11.9 \mathrm{~Hz},{ }^{3} \mathrm{~J}_{2 \mathrm{~A}-1}=7.4 \mathrm{~Hz}, 2 \mathrm{H}, 2-\mathrm{H}_{\mathrm{A}}\right), 3.28\left(\mathrm{dd},{ }^{2} \mathrm{~J}_{2 \mathrm{~B}-2 \mathrm{~A}}=11.9 \mathrm{~Hz},{ }^{3} \mathrm{~J}_{2 \mathrm{~B}^{\prime}-1}=6.4 \mathrm{~Hz}, 2 \mathrm{H}\right.$, $\left.2-\mathrm{H}_{\mathrm{B}}\right), 2.92(\mathrm{~s}, 3 \mathrm{H}, 3-\mathrm{H})$.

${ }^{13} \mathrm{C}-\mathrm{NMR}\left(101 \mathrm{MHz}\right.$, DMSO- $\left.d_{6}, 373 \mathrm{~K}\right): \delta(\mathrm{ppm})=170.2(C=\mathrm{O}, \mathrm{C} 4), 136.1\left(C_{\mathrm{Ar}}, \mathrm{C}\right), 128.8\left(C_{\mathrm{Ar}} \mathrm{H}, \mathrm{C} 8\right), 127.8\left(C_{\mathrm{Ar}} \mathrm{H}\right.$, C6), $126.1\left(\mathrm{C}_{\mathrm{Ar}} \mathrm{H}, \mathrm{C} 7\right), 61.7\left(\mathrm{CH}_{2}, \mathrm{C} 1\right), 39.0\left(\mathrm{CH}_{2}, \mathrm{C} 2\right), 30.1\left(\mathrm{CH}_{2}, \mathrm{C} 3\right)$.

\section{(1,2,5-dithiazepan-5-yl)(phenyl)methanone (SS7-Bz, 69)}<smiles>O=C(c1ccccc1)N1CCSCC1</smiles>

Prepared from crude 49 (corresponding to $200 \mathrm{mg}, 0.321 \mathrm{mmol}$ of compound 47 ) and $\mathrm{BzCl}$ (2.0 eq.) according to general protocol H. Purification by FCC (isohexane/EtOAc) gave 68 (6.4 mg, $0.027 \mathrm{mmol}, 8 \%$ ) as a solid.

TLC $\mathrm{R}_{f}=0.33$ (isohexane:EtOAc, 2:1). HRMS (EI): $\mathrm{C}_{11} \mathrm{H}_{13} \mathrm{NOS}_{2}$ [M-e] calc. 239.0434, found 239.0432.

Individual rotamers were observed by NMR spectroscopy at $298 \mathrm{~K}$ (ratio ca. 1:1) and measurements at $373 \mathrm{~K}$ were not sufficient to obtain time-averaged spectra.

${ }^{1} \mathrm{H}-\mathrm{NMR}\left(400 \mathrm{MHz}, \mathrm{CDCl}_{3}\right): \delta(\mathrm{ppm})=7.32(\mathrm{br}-\mathrm{s}, 5 \mathrm{H}, 5-\mathrm{H} ; 6-\mathrm{H}, 7-\mathrm{H}), 4.01(\mathrm{t}, J=5.7 \mathrm{~Hz}, 2 \mathrm{H}, 1-\mathrm{H}), 3.61$ (t, $\left.J=5.7 \mathrm{~Hz}, 2 \mathrm{H}, 1^{\prime}-\mathrm{H}\right), 3.11(\mathrm{t}, J=5.7 \mathrm{~Hz}, 2 \mathrm{H}, 2-\mathrm{H}), 2.63\left(\mathrm{t}, J=5.7 \mathrm{~Hz}, 2 \mathrm{H}, 2^{\prime}-\mathrm{H}\right)$.

${ }^{13} \mathrm{C}-\mathrm{NMR}\left(101 \mathrm{MHz}, \mathrm{CDCl}_{3}\right): \delta(\mathrm{ppm})=172.2(\mathrm{C}=\mathrm{O}, \mathrm{C} 3), 136.8\left(C_{\mathrm{Ar}}, \mathrm{C} 4\right), 129.6\left(\mathrm{C}_{\mathrm{Ar}} \mathrm{H}, \mathrm{C} 7\right), 128.8\left(C_{\mathrm{Ar}} \mathrm{H}, \mathrm{C} 5\right), 126.2$ $\left(\mathrm{C}_{\mathrm{Ar}} \mathrm{H}, \mathrm{C} 6\right)$, 53.6 $\left(\mathrm{CH}_{2}, \mathrm{C} 1\right)$, $\left.49.7\left(\mathrm{CH}_{2}, \mathrm{C}^{\prime}\right), 40.2\left(\mathrm{CH}_{2}, \mathrm{C} 2\right), 25.4\left(\mathrm{CH}_{2}, \mathrm{C}\right)^{\prime}\right)$. 


\section{$\underline{P Q-S e r i e s}$}

trans-chloro-2-(chloro-4-oxo-3,4-dihydroquinazolin-2-yl)phenyl trans-hexahydro-[1,2]dithiino [4,5-b]pyridine-1(2H)-carboxylate (SS66T-PQ, 70)

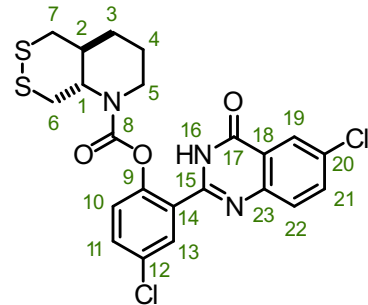

Prepared from $8(57.9 \mathrm{mg}, 0.273 \mathrm{mmol})$ and $55(70.0 \mathrm{mg}, 0.228 \mathrm{mmol})$ according to general protocol G. Purification by FCC (isohexane/EtOAc) gave 70 (75.0 mg, $0.148 \mathrm{mmol}, 65 \%)$ as a colourless solid.

TLC $\mathrm{R}_{f}=0.44$ (isohexane:EtOAc, 2:1). HRMS $\left(E S I^{+}\right.$): for $\mathrm{C}_{22} \mathrm{H}_{20} \mathrm{Cl}_{2} \mathrm{~N}_{3} \mathrm{O}_{3} \mathrm{~S}_{2}{ }^{+}[\mathrm{M}+\mathrm{H}]^{+}$: calc. 508.03176, found 508.03187.

Individual rotamers were observed by NMR spectroscopy at $298 \mathrm{~K}$ (ratio ca. 1:1) and time-averaged spectra were obtained at $373 \mathrm{~K}$.

${ }^{1} \mathrm{H}-N M R \quad\left(400 \mathrm{MHz}\right.$, tetrachlorethane- $\left.d_{2}, 373 \mathrm{~K}\right): \delta(\mathrm{ppm})=8.23\left(\mathrm{t},{ }^{4} \mathrm{~J}_{19-21}=1.5 \mathrm{~Hz}, 1 \mathrm{H}, 19-\mathrm{H}\right), 7.95(\mathrm{~d}$, $\left.J_{13-11}=2.6 \mathrm{~Hz}, 1 \mathrm{H}, 13-\mathrm{H}\right), 7.76-7.67(\mathrm{~m}, 1 \mathrm{H}, 21-\mathrm{H} ; 22-\mathrm{H}), 7.49\left(\mathrm{dd},{ }^{3} J_{11-10}=8.7 \mathrm{~Hz},{ }^{4} J_{11-13}=2.6 \mathrm{~Hz}, 1 \mathrm{H}, 11-\mathrm{H}\right)$, $7.15\left(\mathrm{~d},{ }^{3} J_{10-11}=8.7 \mathrm{~Hz}, 1 \mathrm{H}, 10-\mathrm{H}\right), 3.77\left(\mathrm{ddd},{ }^{2} J_{5 \mathrm{~A}-5 \mathrm{~B}}=13.6 \mathrm{~Hz},{ }^{3} J_{5 \mathrm{~A}-4}=6.8 \mathrm{~Hz},{ }^{3} J_{5 \mathrm{~A}-4 \mathrm{~B}}=3.3 \mathrm{~Hz}, 1 \mathrm{H}, 5-\mathrm{H}_{\mathrm{A}}\right), 3.72$ (ddd, ${ }^{3} J_{1-2}=11.0 \mathrm{~Hz},{ }^{3} J_{1-6 \mathrm{~A}}=10.5 \mathrm{~Hz},{ }^{3} J_{1-6 \mathrm{~B}}=3.2 \mathrm{~Hz}, 1 \mathrm{H}, 1-\mathrm{H}$ ), 3.38 (ddd, ${ }^{2} J_{5 \mathrm{~B}-5 \mathrm{~A}}=14.0 \mathrm{~Hz},{ }^{3} J_{5 \mathrm{~B}-4}=10.2 \mathrm{~Hz}$, $\left.{ }^{3} J_{5-4 \mathrm{~B}}=5.5 \mathrm{~Hz}, 1 \mathrm{H}, 5-\mathrm{H}_{\mathrm{B}}\right), 3.11\left(\mathrm{dd},{ }^{2} J_{6 \mathrm{~A}-6 \mathrm{~B}}=12.9 \mathrm{~Hz},{ }^{3} J_{6 \mathrm{~A}-1}=10.3 \mathrm{~Hz}, 1 \mathrm{H}, 6-\mathrm{H}_{\mathrm{A}}\right), 3.03\left(\mathrm{dd},{ }^{2} J_{6 \mathrm{~B}-6 \mathrm{~A}}=13.0 \mathrm{~Hz}\right.$, $\left.{ }^{3} J_{6 \mathrm{~B}-1}=3.3 \mathrm{~Hz}, 1 \mathrm{H}, 6-\mathrm{H}_{\mathrm{B}}\right), 2.87\left(\mathrm{dd},{ }^{2} J_{7 \mathrm{~A}-7 \mathrm{~B}}=13.6 \mathrm{~Hz},{ }^{3} J_{7 \mathrm{~A}-2}=10.7 \mathrm{~Hz}, 1 \mathrm{H}, 7-\mathrm{H}_{\mathrm{A}}\right), 2.75\left(\mathrm{dd},{ }^{2} J_{7 \mathrm{~B}-7 \mathrm{~A}}=13.5 \mathrm{~Hz}\right.$, $\left.{ }^{3} J_{7 \mathrm{~B}-2}=3.0 \mathrm{~Hz}, 1 \mathrm{H}, 7-\mathrm{H}_{\mathrm{B}}\right), 2.07\left(\mathrm{ddd},{ }^{3} J_{2-1}=13.5 \mathrm{~Hz},{ }^{3} J_{2-7 \mathrm{~A}}=10.7 \mathrm{~Hz}, 1 \mathrm{H}, 2-\mathrm{H}\right.$ ), 1.86 (ddd, ${ }^{2} J_{4 \mathrm{~A}-4 \mathrm{~B}}=11.3 \mathrm{~Hz}$, $\left.{ }^{3} J_{4 \mathrm{~A}-5 \mathrm{~B}}=7.6 \mathrm{~Hz}, 1 \mathrm{H}, 4-\mathrm{H}_{\mathrm{A}}\right), 1.70\left(\mathrm{ddd},{ }^{2} \mathrm{~J}_{4 \mathrm{~B}-4 \mathrm{~A}}=12.4 \mathrm{~Hz},{ }^{3} \mathrm{~J}_{4 \mathrm{~B}-5 \mathrm{~B}}=3.4 \mathrm{~Hz}, 1 \mathrm{H}, 4-\mathrm{H}_{\mathrm{B}}\right), 1.66-1.55\left(\mathrm{~m}, 1 \mathrm{H}, 3-\mathrm{H}_{\mathrm{A}}\right), 1.39$ $-1.22\left(\mathrm{~m}, 1 \mathrm{H}, 3-\mathrm{H}_{\mathrm{B}}\right)$.

${ }^{13} \mathrm{C}-\mathrm{NMR}\left(101 \mathrm{MHz}\right.$, tetrachlorethane- $\left.d_{2}, 373 \mathrm{~K}\right): \delta(\mathrm{ppm})=160.4(C=\mathrm{O}, \mathrm{C} 17), 153.3(C=\mathrm{O}, \mathrm{C} 8), 149.0(C=\mathrm{N}, \mathrm{C} 15)$, $147.1\left(C_{\mathrm{Ar}}, \mathrm{C} 18\right), 135.3\left(C_{\mathrm{Ar}} \mathrm{H}, \mathrm{C} 22\right), 133.2\left(C_{\mathrm{Ar}}, \mathrm{C} 23\right), 132.3\left(C_{\mathrm{Ar}} \mathrm{H}, \mathrm{C} 11\right), 131.9\left(C_{\mathrm{Ar}}, \mathrm{C} 12\right), 130.3\left(C_{\mathrm{Ar}} \mathrm{H}, \mathrm{C} 13\right)$, $129.5\left(C_{\mathrm{Ar}} \mathrm{H}, \mathrm{C} 21\right), 128.0\left(C_{\mathrm{Ar}}, \mathrm{C} 14\right), 125.8\left(C_{\mathrm{Ar}} \mathrm{H}, \mathrm{C} 19\right), 125.3\left(C_{\mathrm{Ar}} \mathrm{H}, \mathrm{C} 10\right), 121.9\left(C_{\mathrm{Ar}}, \mathrm{C} 20\right), 63.1(C H, C 1), 40.7$ $\left(\mathrm{CH}_{2}, \mathrm{C} 7\right), 40.2(\mathrm{CH}, \mathrm{C} 2), 39.5\left(\mathrm{CH}_{2}, \mathrm{C} 5\right), 36.2\left(\mathrm{CH}_{2}, \mathrm{C} 6\right), 26.9\left(\mathrm{CH}_{2}, \mathrm{C} 3\right), 23.1\left(\mathrm{CH}_{2}, \mathrm{C} 4\right)$.

\section{cis-chloro-2-(chloro-4-oxo-3,4-dihydroquinazolin-2-yl)phenyl-hexahydro-[1,2]dithiino} $[4,5-b]$ pyridine-1 $(2 H)$-carboxylate (SS66C-PQ, 71)

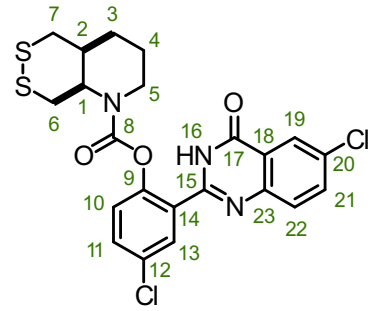

Prepared from crude 18 (corresponding to $41.5 \mathrm{mg}, 0.140 \mathrm{mmol}$ of 12) and $\mathbf{5 5}$ (51.6 $\mathrm{mg}, 0.168 \mathrm{mmol}$ ) according to general protocol G. Purification by FCC (isohexane/EtOAc) gave 71 (28.4 mg, $0.056 \mathrm{mmol}, 40 \%)$ as a colourless solid.

TLC $\mathrm{R}_{f}=0.40$ (isohexane:EtOAc, 2:1). HRMS $\left(E S I^{+}\right): \mathrm{C}_{22} \mathrm{H}_{20} \mathrm{Cl}_{2} \mathrm{~N}_{3} \mathrm{O}_{3} \mathrm{~S}_{2}{ }^{+}[\mathrm{M}+\mathrm{H}]^{+}$: calc. 508.03176, found 508.03186.

Individual rotamers were observed by NMR spectroscopy at $298 \mathrm{~K}$ (ratio ca. 1.2:1). The following data refers to the major rotamer, as indicated based on 2D-NMR analysis. 
${ }^{1} \mathrm{H}-\mathrm{NMR}\left(500 \mathrm{MHz}, \mathrm{CDCl}_{3}\right): \delta(\mathrm{ppm})=10.15(\mathrm{~s}, 1 \mathrm{H}, 16-\mathrm{H}), 8.26\left(\mathrm{t},{ }^{4} J_{19-21}=2.3 \mathrm{~Hz}, 1 \mathrm{H}, 19-\mathrm{H}\right), 7.99(\mathrm{~d}$, $\left.{ }^{4} J_{13-11}=2.6 \mathrm{~Hz}, 1 \mathrm{H}, 13-\mathrm{H}\right), 7.80-7.67(\mathrm{~m}, 2 \mathrm{H}, 21-\mathrm{H} ; 22-\mathrm{H}), 7.51\left(\mathrm{dt},{ }^{3} \mathrm{~J}_{11-10}=8.8 \mathrm{~Hz},{ }^{4} \mathrm{~J}_{11-13}=2.7 \mathrm{~Hz}, 1 \mathrm{H}, 11-\mathrm{H}\right)$, $7.17\left(\mathrm{~d},{ }^{3} J_{10-11}=6.8 \mathrm{~Hz}, 1 \mathrm{H}, 10-\mathrm{H}\right), 4.50\left(\mathrm{dt},{ }^{3} J_{1-6 \mathrm{~A}}=12.1 \mathrm{~Hz},{ }^{3} J_{1-6 \mathrm{~B}}=4.2 \mathrm{~Hz}, 1 \mathrm{H}, 1-\mathrm{H}\right), 4.15\left(\mathrm{dd},{ }^{2} J_{5 \mathrm{~A}-5 \mathrm{~B}}=13.9 \mathrm{~Hz}\right.$, $\left.{ }^{3} J_{5 \mathrm{~A}-4 \mathrm{~A}}=4.6 \mathrm{~Hz}, 1 \mathrm{H}, 5-\mathrm{H}_{\mathrm{A}}\right), 3.47\left(\mathrm{dd},{ }^{2} J_{7 \mathrm{~A}-7 \mathrm{~B}}=13.9 \mathrm{~Hz},{ }^{3} \mathrm{~J}_{7 \mathrm{~A}-2}=6.2 \mathrm{~Hz}, 1 \mathrm{H}, 7-\mathrm{H}_{\mathrm{A}}\right), 3.41\left(\mathrm{t},{ }^{2} J_{6 \mathrm{~A}-6 \mathrm{~B}}=13.0 \mathrm{~Hz}\right.$, $\left.{ }^{3} J_{6 \mathrm{~A}-1}=12.1 \mathrm{~Hz}, 1 \mathrm{H}, 6-\mathrm{H}_{\mathrm{A}}\right), 3.07\left(\mathrm{td},{ }^{2} J_{5 \mathrm{~B}-5 \mathrm{~A}}=13.5 \mathrm{~Hz},{ }^{3} J_{5 \mathrm{~B}-4 \mathrm{~A}}=3.1 \mathrm{~Hz}, 1 \mathrm{H}, 5-\mathrm{H}_{\mathrm{B}}\right), 2.79\left(\mathrm{td},{ }^{2} J_{7 \mathrm{~B}-7 \mathrm{~A}^{\mathrm{A}}}=13.9 \mathrm{~Hz}\right.$, $\left.{ }^{3} J_{7 \mathrm{~B}-2}=13.2 \mathrm{~Hz}, 1 \mathrm{H}, 7-\mathrm{H}_{\mathrm{B}}\right), 2.39\left(\mathrm{dd},{ }^{2} \mathrm{~J}_{6 \mathrm{~B}-6 \mathrm{~A}}=13.1 \mathrm{~Hz},{ }^{3} \mathrm{~J}_{6 \mathrm{~B}-1}=3.9 \mathrm{~Hz}, 1 \mathrm{H}, 6-\mathrm{H}_{\mathrm{B}}\right), 2.30\left(\mathrm{dd},{ }^{2} \mathrm{~J}_{3 \mathrm{~A}-3 \mathrm{~B}}=12.9 \mathrm{~Hz}\right.$, $\left.{ }^{3} J_{3 \mathrm{~A}-2}=4.0 \mathrm{~Hz}, 1 \mathrm{H}, 3-\mathrm{H}_{\mathrm{A}}\right), 2.20\left(\mathrm{dt},{ }^{3} J_{2-7 \mathrm{~B}}=12.5 \mathrm{~Hz},{ }^{3} J_{2-7 \mathrm{~A}}=3.7 \mathrm{~Hz}, 1 \mathrm{H}, 2-\mathrm{H}\right), 1.83\left(\mathrm{t},{ }^{2} J_{4 \mathrm{~A}-4 \mathrm{~B}}=13.6 \mathrm{~Hz}, 1 \mathrm{H}, 4-\mathrm{H}_{\mathrm{A}}\right)$, $1.68-1.55\left(\mathrm{~m}, 1 \mathrm{H}, 4-\mathrm{H}_{\mathrm{B}}\right), 1.49\left(\mathrm{~d},{ }^{2} \mathrm{~J}_{3 \mathrm{~B}-3 \mathrm{~A}}=13.3 \mathrm{~Hz}, 1 \mathrm{H}, 3-\mathrm{H}_{\mathrm{B}}\right)$.

${ }^{13} \mathrm{C}-\mathrm{NMR}\left(101 \mathrm{MHz}, \mathrm{CDCl}_{3}\right): \delta(\mathrm{ppm})=160.7(C=\mathrm{O}, \mathrm{C} 17), 153.3(C=\mathrm{O}, \mathrm{C} 8), 149.3(C=\mathrm{N}, \mathrm{C} 15), 147.6\left(C_{\mathrm{Ar}}, \mathrm{C} 9\right)$, $147.5\left(C_{\mathrm{A}}, \mathrm{C} 18\right), 135.5\left(C_{\mathrm{Ar}}, \mathrm{C} 22\right), 133.6\left(C_{\mathrm{Ar}}, \mathrm{C} 23\right), 132.5\left(C_{\mathrm{Ar}} \mathrm{H}, \mathrm{C} 11\right), 132.4\left(C_{\mathrm{Ar}}, \mathrm{C} 12\right), 130.7\left(C_{\mathrm{Ar}} \mathrm{H}, \mathrm{C} 13\right), 129.8$ $\left(C_{\mathrm{Ar}} \mathrm{H}, \mathrm{C} 21\right), 128.1\left(C_{\mathrm{Ar}}, \mathrm{C} 14\right), 126.2\left(C_{\mathrm{Ar}} \mathrm{H}, \mathrm{C} 19\right), 125.3\left(C_{\mathrm{Ar}} \mathrm{H}, \mathrm{C} 10\right), 122.3\left(C_{\mathrm{Ar}}, \mathrm{C} 20\right), 53.3(\mathrm{CH}, \mathrm{C} 1), 42.1\left(\mathrm{CH}_{2}\right.$, C7), $40.7\left(\mathrm{CH}_{2}, \mathrm{C} 5\right), 35.2(\mathrm{CH}, \mathrm{C} 2), 29.3\left(\mathrm{CH}_{2}, \mathrm{C} 6\right), 26.3\left(\mathrm{CH}_{2}, \mathrm{C} 3\right), 23.4\left(\mathrm{CH}_{2}, \mathrm{C} 4\right)$.

Time-averaged spectra were obtained at $373 \mathrm{~K}$.

${ }^{1} \mathrm{H}-N M R\left(400 \mathrm{MHz}\right.$, tetrachlorethan- $\left.d_{2}, 373 \mathrm{~K}\right): \delta(\mathrm{ppm})=9.72(\mathrm{~s}, 1 \mathrm{H}, 16-\mathrm{H}), 8.23\left(\mathrm{t},{ }^{4} \mathrm{~J}_{19-21}=1.5 \mathrm{~Hz}, 1 \mathrm{H}, 19-\mathrm{H}\right)$, $7.99\left(\mathrm{~d},{ }^{4} J_{13-11}=2.6 \mathrm{~Hz}, 1 \mathrm{H}, 13-\mathrm{H}\right), 7.73(\mathrm{~m}, 2 \mathrm{H}, 21-\mathrm{H} ; 22-\mathrm{H}), 7.50\left(\mathrm{dd},{ }^{3} J_{11-10}=8.7 \mathrm{~Hz},{ }^{4} J_{11-13}=2.6 \mathrm{~Hz}, 1 \mathrm{H}\right), 7.17$ $\left(\mathrm{d},{ }^{3} J_{10-11}=8.7 \mathrm{~Hz}, 1 \mathrm{H}, 10-\mathrm{H}\right), 4.48\left(\mathrm{~d},{ }^{3} J_{1-6 \mathrm{~A}}=11.7 \mathrm{~Hz}, 1 \mathrm{H}, 1-\mathrm{H}\right), 4.07\left(\mathrm{~d},{ }^{2} J_{5 \mathrm{~A}-5 \mathrm{~B}}=12.5 \mathrm{~Hz}, 1 \mathrm{H}, 5-\mathrm{H}_{\mathrm{A}}\right), 3.53-3.33$ $\left(\mathrm{m}, 2 \mathrm{H}, 6-\mathrm{H}_{\mathrm{A}} ; 7-\mathrm{H}_{\mathrm{A}}\right), 3.18-2.88\left(\mathrm{~m}, 1 \mathrm{H}, 5-\mathrm{H}_{\mathrm{A}}\right), 2.77\left(\mathrm{dd},{ }^{2} J_{7 \mathrm{~B}-7 \mathrm{~A}}=14.0 \mathrm{~Hz}, J=3.0 \mathrm{~Hz}, 1 \mathrm{H}, 7-\mathrm{H}_{\mathrm{B}}\right), 2.43(\mathrm{~d}$, $\left.{ }^{2} J_{6 \mathrm{~B}-6 \mathrm{~A}}=12.7 \mathrm{~Hz}, 1 \mathrm{H}, 6-\mathrm{H}_{\mathrm{B}}\right), 2.28\left(\mathrm{dd},{ }^{2} \mathrm{~J}_{3 \mathrm{~A}-3 \mathrm{~B}}=12.7 \mathrm{~Hz}, J=4.0 \mathrm{~Hz}, 1 \mathrm{H}, 3-\mathrm{H}_{\mathrm{A}}\right), 2.25-2.17(\mathrm{~m}, 1 \mathrm{H}, 2-\mathrm{H}), 1.81(\mathrm{~d}$, $\left.{ }^{2} J_{4 \mathrm{~A}-4 \mathrm{~B}}=13.6 \mathrm{~Hz}, 1 \mathrm{H}, 4-\mathrm{H}_{\mathrm{A}}\right), 1.70-1.54\left(\mathrm{~m}, 1 \mathrm{H}, 4-\mathrm{H}_{\mathrm{B}}\right), 1.47\left(\mathrm{~d},{ }^{2} J_{3 \mathrm{~B}-3 \mathrm{~A}}=13.1 \mathrm{~Hz}, 1 \mathrm{H}, 3-\mathrm{H}_{\mathrm{B}}\right)$.

${ }^{13} \mathrm{C}-\mathrm{NMR}\left(101 \mathrm{MHz}\right.$, tetrachlorethan- $\left.d_{2}, 373 \mathrm{~K}\right): \delta(\mathrm{ppm})=160.2(C=\mathrm{O}, \mathrm{C} 17), 154.9(C=\mathrm{O}, \mathrm{C}), 149.1(C=\mathrm{N}, \mathrm{C} 15)$, $147.7\left(C_{\mathrm{Ar}}, \mathrm{C} 19\right), 147.5\left(C_{\mathrm{Ar}}, \mathrm{C} 18\right), 135.3\left(C_{\mathrm{Ar}} \mathrm{H}, \mathrm{C} 22\right), 133.5\left(C_{\mathrm{Ar}}, \mathrm{C} 23\right), 132.4\left(C_{\mathrm{Ar}} \mathrm{H}, \mathrm{C} 11\right), 132.3\left(C_{\mathrm{Ar}}, \mathrm{C} 12\right), 130.5$ ( $\left.C_{\mathrm{Ar}} \mathrm{H}, \mathrm{C} 13\right), 129.9\left(C_{\mathrm{Ar}} \mathrm{H}, \mathrm{C} 21\right), 128.0\left(C_{\mathrm{Ar}}, \mathrm{C} 14\right), 126.1\left(C_{\mathrm{Ar}} \mathrm{H}, \mathrm{C} 18\right), 125.4\left(C_{\mathrm{Ar}} \mathrm{H}, \mathrm{C} 10\right), 122.4\left(C_{\mathrm{Ar}}, \mathrm{C} 20\right), 54.6$ $(\mathrm{CH}, \mathrm{C} 1), 42.2\left(\mathrm{CH}_{2}, \mathrm{C} 7\right), 41.3\left(\mathrm{CH}_{2}, \mathrm{C} 5\right), 36.0(\mathrm{CH}, \mathrm{C} 2), 29.7\left(\mathrm{CH}_{2}, \mathrm{C} 6\right), 25.3\left(\mathrm{CH}_{2}, \mathrm{C} 3\right), 23.1\left(\mathrm{CH}_{2}, \mathrm{C} 4\right)$.

\section{cis-chloro-2-(chloro-4-oxo-3,4-dihydroquinazolin-2-yl)phenyl-hexahydrofuro-[3,4-b]pyridine- $1(2 H)$-carboxylate $(\mathrm{O} 56-\mathrm{PQ}, 72)$}

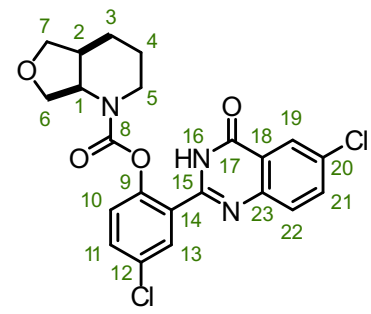

Prepared from 19 (92 mg, $0.56 \mathrm{mmol})$ and 55 (345 mg, $1.12 \mathrm{mmol})$ according to general protocol G. Purification by FCC $\left(\mathrm{Et}_{2} \mathrm{O}\right)$ gave 72 (199 $\left.\mathrm{mg}, 0.432 \mathrm{mmol}, 77 \%\right)$ as a colourless solid.

TLC $\mathrm{R}_{f}=0.51\left(\mathrm{Et}_{2} \mathrm{O}\right)$. HRMS $\left(\mathrm{ESI}^{+}\right): \mathrm{C}_{22} \mathrm{H}_{20} \mathrm{Cl}_{2} \mathrm{~N}_{3} \mathrm{O}_{4}{ }^{+}[\mathrm{M}+\mathrm{H}]^{+}$: calc. 460.08254, found 460.08240.

Individual rotamers were observed by NMR spectroscopy at $298 \mathrm{~K}$ (ratio ca. 1.2:1) and time-averaged spectra were obtained at $373 \mathrm{~K}$.

${ }^{1} \mathrm{H}-\mathrm{NMR}\left(400 \mathrm{MHz}\right.$, tetrachlorethane- $\left.\mathrm{d}_{2}, 373 \mathrm{~K}\right): \delta(\mathrm{ppm})=9.65(\mathrm{~s}, 1 \mathrm{H}, 16-\mathrm{H}), 8.23(\mathrm{t}, \mathrm{J}=1.5 \mathrm{~Hz}, 1 \mathrm{H}, 19-\mathrm{H}), 7.96$ $(\mathrm{d}, J=2.6 \mathrm{~Hz}, 1 \mathrm{H}, 13-\mathrm{H}), 7.78-7.63(\mathrm{~m}, 2 \mathrm{H}, 21-\mathrm{H} ; 22-\mathrm{H}), 7.48(\mathrm{dd}, J=8.7,2.6 \mathrm{~Hz}, 1 \mathrm{H}, 11-\mathrm{H}), 7.17(\mathrm{~d}, J=8.7 \mathrm{~Hz}$, $1 \mathrm{H}, 10-\mathrm{H}), 4.69$ (q, J = 8.0 Hz, 1H, 1-H), 3.99 (dd, $\left.J=13.7,4.4 \mathrm{~Hz}, 1 \mathrm{H}, 5-\mathrm{H}_{\mathrm{A}}\right), 3.90\left(\mathrm{t}, J=8.5 \mathrm{~Hz}, 1 \mathrm{H}, 6-\mathrm{H}_{\mathrm{A}}\right), 3.83$ (dd, $J=8.8,5.4 \mathrm{~Hz}, 1 \mathrm{H}, 7-\mathrm{H}_{\mathrm{A}}$ ), $3.63\left(\mathrm{dd}, J=8.8,1.9 \mathrm{~Hz}, 1 \mathrm{H}, 7-\mathrm{H}_{\mathrm{B}}\right), 3.63\left(\mathrm{dd}, J=9.0,8.5 \mathrm{~Hz}, 1 \mathrm{H}, 6-\mathrm{H}_{\mathrm{B}}\right), 3.07$ (t, $\left.J=12.9 \mathrm{~Hz}, 1 \mathrm{H}, 5-\mathrm{H}_{\mathrm{B}}\right), 2.25(\mathrm{tq}, J=7.4,2.8,2.1 \mathrm{~Hz}, 1 \mathrm{H}, 2-\mathrm{H}), 1.86-1.69\left(\mathrm{~m}, 2 \mathrm{H}, 3-\mathrm{H}_{\mathrm{A}} ; 4-\mathrm{H}_{\mathrm{A}}\right), 1.57-1.44(\mathrm{~m}, 2 \mathrm{H}$, $\left.3-\mathrm{H}_{\mathrm{B}} ; 4-\mathrm{H}_{\mathrm{B}}\right)$

${ }^{13} \mathrm{C}-N M R\left(101 \mathrm{MHz}\right.$, tetrachlorethan- $\left.d_{2}, 373 \mathrm{~K}\right): \delta(\mathrm{ppm})=160.1(C=\mathrm{O}, \mathrm{C} 17), 153.7(C=\mathrm{O}, \mathrm{C} 8), 149.1(C=\mathrm{N}, \mathrm{C} 15)$, $147.8\left(C_{\mathrm{Ar}}, \mathrm{C} 9\right), 147.5\left(C_{\mathrm{Ar}}, \mathrm{C} 18\right), 135.1\left(C_{\mathrm{Ar}} \mathrm{H}, \mathrm{C} 22\right), 133.4\left(C_{\mathrm{Ar}}, \mathrm{C} 23\right), 132.2\left(C_{\mathrm{Ar}} \mathrm{H}, \mathrm{C} 11\right), 132.1\left(C_{\mathrm{Ar}}, \mathrm{C} 12\right), 130.4$ $\left(C_{A r} \mathrm{H}, \mathrm{C} 13\right), 129.8\left(C_{\mathrm{Ar}} \mathrm{H}, \mathrm{C} 21\right), 128.0\left(C_{\mathrm{Ar}}, \mathrm{C} 14\right), 126.0\left(C_{\mathrm{Ar}} \mathrm{H}, \mathrm{C} 19\right), 125.1\left(C_{\mathrm{Ar}} \mathrm{H}, \mathrm{C} 10\right), 122.4\left(C_{\mathrm{Ar}}, \mathrm{C} 20\right), 73.2$ $\left(\mathrm{CH}_{2}, \mathrm{C} 7\right), 67.1\left(\mathrm{CH}_{2}, \mathrm{C} 6\right), 54.8(\mathrm{CH}, \mathrm{C} 1), 41.3\left(\mathrm{CH}_{2}, \mathrm{C} 5\right), 36.0(\mathrm{CH}, \mathrm{C} 2), 24.6\left(\mathrm{CH}_{2}, \mathrm{C} 3\right), 22.8\left(\mathrm{CH}_{2}, \mathrm{C} 4\right)$. 


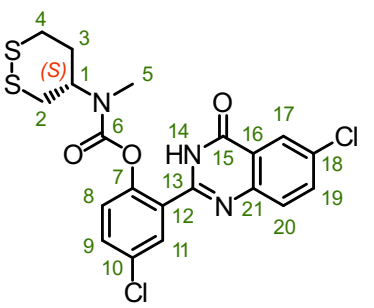

Prepared from 25 (56 mg, $0.30 \mathrm{mmol})$ and $55(180 \mathrm{mg}, 0.586 \mathrm{mmol})$ according to general protocol G. Purification by FCC $\left(\mathrm{Et}_{2} \mathrm{O}\right)$ gave $73(108 \mathrm{mg}, 0.224 \mathrm{mmol}, 75 \%)$ as a colourless solid.

TLC $\mathrm{R}_{f}=0.31$ (isohexane:EtOAc, 2:1). $\mathrm{HRMS}\left(\mathrm{ESI}^{+}\right): \mathrm{C}_{20} \mathrm{H}_{18} \mathrm{Cl}_{2} \mathrm{~N}_{3} \mathrm{O}_{3} \mathrm{~S}_{2}{ }^{+}[\mathrm{M}+\mathrm{H}]^{+}$calc. 482.01611, found 482.01592. Individual rotamers were observed by NMR spectroscopy at $298 \mathrm{~K}$ (ratio ca. 1.5:1) and time-averaged spectra were obtained at $373 \mathrm{~K}$.

${ }^{1} \mathrm{H}-\mathrm{NMR}\left(400 \mathrm{MHz}\right.$, tetrachlorethane- $\left.\mathrm{d}_{2}, 373 \mathrm{~K}\right): \delta(\mathrm{ppm})=9.56(\mathrm{~s}, 1 \mathrm{H}, 14-\mathrm{H}), 8.22(\mathrm{t}, \mathrm{J}=1.5 \mathrm{~Hz}, 1 \mathrm{H}, 17-\mathrm{H}), 7.97$ $(\mathrm{d}, J=2.6 \mathrm{~Hz}, 1 \mathrm{H}, 11-\mathrm{H}), 7.78-7.66(\mathrm{~m}, 2 \mathrm{H}, 19-\mathrm{H} ; 20-\mathrm{H}), 7.49(\mathrm{dd}, J=8.7,2.6 \mathrm{~Hz}, 1 \mathrm{H}, 9-\mathrm{H}), 7.17(\mathrm{~d}, J=8.8 \mathrm{~Hz}$, $1 \mathrm{H}, 8-\mathrm{H}), 4.17(\mathrm{~d}, J=12.1 \mathrm{~Hz}, 1 \mathrm{H}, 1-\mathrm{H}), 3.15\left(\mathrm{ddd}, J=13.8,11.8,2.8 \mathrm{~Hz}, 1 \mathrm{H}, 3-\mathrm{H}_{\mathrm{B}}\right), 3.10(\mathrm{dd}, J=12.9,11.4 \mathrm{~Hz}$, $\left.1 \mathrm{H}, 2-\mathrm{H}_{\mathrm{A}}\right), 2.97(\mathrm{~s}, 3 \mathrm{H}, 5-\mathrm{H}), 2.93\left(\mathrm{t}, J=3.8 \mathrm{~Hz}, 1 \mathrm{H}, 3-\mathrm{H}_{\mathrm{B}}\right), 2.72\left(\mathrm{dd}, J=12.8,3.2 \mathrm{~Hz}, 1 \mathrm{H}, 2-\mathrm{H}_{\mathrm{B}}\right), 2.17-2.01(\mathrm{~m}$, $2 \mathrm{H}, 4-\mathrm{H})$.

${ }^{13} \mathrm{C}$-NMR (101 MHz, tetrachlorethan- $\left.d_{2}, 373 \mathrm{~K}\right): \delta(\mathrm{ppm})=160.0(C=\mathrm{O}, \mathrm{C} 15), 153.4(C=\mathrm{O}, \mathrm{C} 6), 149.0(C=\mathrm{N}, \mathrm{C} 13)$, $147.7\left(C_{\mathrm{Ar}}, \mathrm{C} 7\right), 147.5\left(C_{\mathrm{Ar}}, \mathrm{C} 16\right), 135.2\left(C_{\mathrm{Ar}} \mathrm{H}, \mathrm{C} 20\right), 133.5\left(C_{\mathrm{Ar}}, \mathrm{C} 21\right), 132.3\left(C_{\mathrm{Ar}} \mathrm{H}, \mathrm{C}\right), 132.3\left(C_{\mathrm{Ar}}, \mathrm{C} 10\right), 130.4$ $\left(C_{\mathrm{Ar}} \mathrm{H}, \mathrm{C} 11\right), 129.8\left(C_{\mathrm{Ar}} \mathrm{H}, \mathrm{C} 19\right), 128.0\left(C_{\mathrm{Ar}}, \mathrm{C} 12\right), 126.0\left(C_{\mathrm{Ar}} \mathrm{H}, \mathrm{C} 17\right), 125.2\left(C_{\mathrm{Ar}} \mathrm{H}, \mathrm{C} 8\right), 122.5\left(C_{\mathrm{Ar}}, \mathrm{C} 18\right), 57.1(\mathrm{CH}$, $\mathrm{C} 1), 36.1\left(\mathrm{CH}_{2}, \mathrm{C} 2\right), 35.9\left(\mathrm{CH}_{2}, \mathrm{C} 3\right), 32.6\left(\mathrm{CH}_{2}, \mathrm{C} 4\right), 30.5\left(\mathrm{CH}_{3}, \mathrm{C} 5\right)$.

\section{4-chloro-2-(6-chloro-4-oxo-3,4-dihydroquinazolin-2-yl)phenyl (1,2-dithiolan-4-yl) (methyl)carbamate (SS50-PQ, 74) $)^{11}$}

Prepared from 52 (56 mg, $0.30 \mathrm{mmol})$ and $55(180 \mathrm{mg}, 0.586 \mathrm{mmol})$ according to general protocol G. Purification by FCC (isohexane/EtOAc) gave $74(39.0 \mathrm{mg}, 0.083 \mathrm{mmol}, 49 \%)$ as a colourless solid.

TLC $\mathrm{R}_{f}=0.26$ (isohexane:EtOAc, 3:1). HRMS $\left(E S I^{+}\right): \mathrm{C}_{19} \mathrm{H}_{16} \mathrm{Cl}_{2} \mathrm{~N}_{3} \mathrm{O}_{3} \mathrm{~S}_{2}{ }^{+}[\mathrm{M}+\mathrm{H}]^{+}$: calc. 468.00046, found 468.00049. ${ }^{1} \mathrm{H}-\mathrm{NMR}\left(400 \mathrm{MHz}\right.$, tetrachlorethan- $\left.\mathrm{d}_{2}, 373 \mathrm{~K}\right): \delta(\mathrm{ppm})=8.24-8.22(\mathrm{~m}, 1 \mathrm{H}), 7.95(\mathrm{~d}, \mathrm{~J}=2.6 \mathrm{~Hz}, 1 \mathrm{H})$, $7.71(\mathrm{~d}, J=1.9 \mathrm{~Hz}, 2 \mathrm{H}), 7.50$ (dd, $J=8.7,2.7 \mathrm{~Hz}, 1 \mathrm{H}), 7.20(\mathrm{~d}, J=8.7 \mathrm{~Hz}, 1 \mathrm{H}), 5.09(\mathrm{p}, J=6.7 \mathrm{~Hz}, 1 \mathrm{H}), 3.32(\mathrm{dd}$, $J=12.1,7.6 \mathrm{~Hz}, 2 \mathrm{H}), 3.10(\mathrm{dd}, J=12.1,5.6 \mathrm{~Hz}, 2 \mathrm{H}), 3.04(\mathrm{~s}, 3 \mathrm{H}) .{ }^{13} \mathrm{C}-\mathrm{NMR}(101 \mathrm{MHz}$, tetrachlorethan-d $2,373 \mathrm{~K})$ : $\delta(\mathrm{ppm})=160.1,153.7,149.0,147.6,147.4,135.3,133.6,132.3,132.1,130.4,129.7,128.0,126.1,125.2,122.4$, $62.9,40.7,30.6$.

\section{chloro-2-(chloro-4-oxo-3,4-dihydroquinazolin-2-yl)phenyl 1,2,5-dithiazepane-carboxylate} (SS7-PQ, 75)
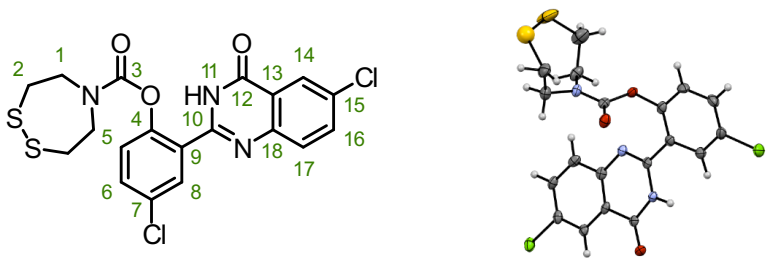

Prepared from crude 49 (corresponding to $400 \mathrm{mg}, 0.642 \mathrm{mmol}$ of 47 ) and an excess of 55 (237 mg, $0.771 \mathrm{mmol}$ ) according to general protocol G to give $75(23 \mathrm{mg}, 0.049 \mathrm{mmol}, 7 \%)$ as a colourless solid after FCC (isohexane/EtOAc). Unambiguous confirmation of the absolute configuration was derived from a single-crystal $X$ Ray analysis.

TLC $\mathrm{R}_{f}=0.32$ (isohexane:EtOAc, 2:1). HRMS $\left(E S I^{+}\right): \mathrm{C}_{19} \mathrm{H}_{16} \mathrm{Cl}_{2} \mathrm{~N}_{3} \mathrm{O}_{3} \mathrm{~S}_{2}{ }^{+}[\mathrm{M}+\mathrm{H}]^{+}$: calc. 466.9932, found 466.9930.

${ }^{1} \mathrm{H}-\mathrm{NMR}\left(400 \mathrm{MHz}, \mathrm{CDCl}_{3}\right): \delta(\mathrm{ppm})=10.04(\mathrm{~s}, 1 \mathrm{H}, 11-\mathrm{H}), 8.28(\mathrm{dd}, J=2.1,0.9 \mathrm{~Hz}, 1 \mathrm{H}, 14-\mathrm{H}), 7.90(\mathrm{~d}, J=2.6 \mathrm{~Hz}$, $1 \mathrm{H}, 8-\mathrm{H}), 7.80-7.65(\mathrm{~m}, 2 \mathrm{H}, 16-\mathrm{H} ; 17-\mathrm{H}), 7.53(\mathrm{dd}, J=8.7,2.6 \mathrm{~Hz}, 1 \mathrm{H}, 6-\mathrm{H}), 7.19(\mathrm{~d}, J=8.7 \mathrm{~Hz}, 1 \mathrm{H}, 5-\mathrm{H}), 3.91$ (dt, $J=5.8 \mathrm{~Hz}, 4 \mathrm{H}, 1-\mathrm{H}), 3.02(\mathrm{dt}, J=30.7,5.7 \mathrm{~Hz}, 4 \mathrm{H}, 2-\mathrm{H})$.

${ }^{13} \mathrm{C}-\mathrm{NMR}\left(126 \mathrm{MHz}, \mathrm{CDCl}_{3}\right): \delta(\mathrm{ppm})=160.6(C=\mathrm{O}, \mathrm{C} 12), 154.4(C=\mathrm{O}, \mathrm{C} 3), 149.4(C=\mathrm{N}, \mathrm{C} 10), 147.4\left(C_{\mathrm{Ar}}, \mathrm{C} 4\right)$, $147.3\left(C_{\mathrm{Ar}}, \mathrm{C} 13\right), 135.4\left(C_{\mathrm{Ar}} \mathrm{H}, \mathrm{C} 17\right), 133.6\left(C_{\mathrm{Ar}}, \mathrm{C} 18\right), 132.6\left(C_{\mathrm{Ar}} \mathrm{H}, \mathrm{C} 6\right), 132.5\left(C_{\mathrm{Ar}}, \mathrm{C} 7\right), 130.7\left(C_{\mathrm{Ar}} \mathrm{H}, \mathrm{C}\right), 129.7$ $\left(C_{\mathrm{Ar}} \mathrm{H}, \mathrm{C} 16\right), 128.9\left(C_{\mathrm{Ar}}, \mathrm{C} 9\right), 126.2\left(C_{\mathrm{Ar}} \mathrm{H}, \mathrm{C} 14\right), 125.3\left(C_{\mathrm{Ar}} \mathrm{H}, \mathrm{C} 5\right), 122.7\left(C_{\mathrm{Ar}}, \mathrm{C} 15\right), 52.5(\mathrm{CH}, \mathrm{C} 1), 51.8\left(\mathrm{CH}, \mathrm{C} 1^{\prime}\right)$, $37.9\left(\mathrm{CH}_{2}, \mathrm{C} 2\right), 36.4\left(\mathrm{CH}_{2}, \mathrm{C} 2\right)$. 


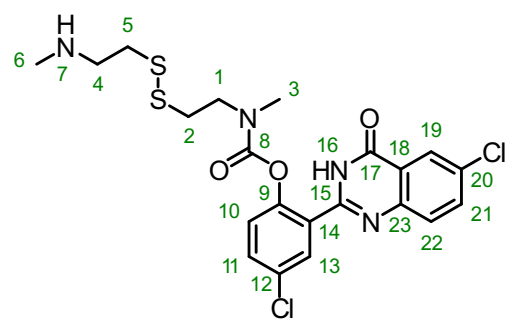

Prepared from $34(715 \mathrm{mg}, 2.82 \mathrm{mmol})$ and $\mathbf{5 5}$ (333 mg, $1.08 \mathrm{mmol})$ according to general protocol $\mathbf{G}$ to give 76 (245 mg, $0.477 \mathrm{mmol}, 44 \%)$ as a colourless solid FCC (EtOAc/MeOH). Precipitation of the hydrochloride salt was achieved by adding $\mathrm{HCl}\left(1 \mathrm{M}\right.$ in $\left.\mathrm{Et}_{2} \mathrm{O}\right)$ to a solution of the residue in $\mathrm{Et}_{2} \mathrm{O}$, followed by filtration to $76 \cdot \mathrm{HCl}$ as a colourless solid.

TLC $\mathrm{R}_{f}=0.22$ (DCM:MeOH, 19:1). HRMS $\left(\mathrm{ESI}^{+}\right): \mathrm{C}_{21} \mathrm{H}_{23} \mathrm{Cl}_{2} \mathrm{~N}_{4} \mathrm{O}_{3} \mathrm{~S}_{2}{ }^{+}[\mathrm{M}+\mathrm{H}]^{+}$: calc. 513.05831, found 513.05886.

${ }^{1} \mathrm{H}-N M R\left(400 \mathrm{MHz}, \mathrm{DMSO}-\mathrm{d}_{6}\right): \delta(\mathrm{ppm})=9.03\left(\mathrm{~s}, 1 \mathrm{H}, 7-\mathrm{H}_{\mathrm{A}}\right), 8.97\left(\mathrm{~s}, 1 \mathrm{H}, 7-\mathrm{H}_{\mathrm{B}}\right), 8.10(\mathrm{~d}, \mathrm{~J}=2.5 \mathrm{~Hz}, 1 \mathrm{H}, 13-\mathrm{H})$, $7.89(\mathrm{dd}, J=8.7,2.2 \mathrm{~Hz}, 1 \mathrm{H}, 11-\mathrm{H}), 7.81(\mathrm{~d}, J=2.1 \mathrm{~Hz}, 1 \mathrm{H}, 19-\mathrm{H}), 7.70(\mathrm{t}, J=8.2 \mathrm{~Hz}, 1 \mathrm{H}, 10-\mathrm{H}), 7.66(\mathrm{dd}, J=8.7$, $2.5 \mathrm{~Hz}, 1 \mathrm{H}, 21-\mathrm{H}), 7.38(\mathrm{dd}, J=11.6,8.8 \mathrm{~Hz}, 1 \mathrm{H}, 22-\mathrm{H}), 3.61\left(\mathrm{t}, J=6.7 \mathrm{~Hz}, 1 \mathrm{H}, 4-\mathrm{H}_{\mathrm{A}}\right), 3.37(\mathrm{t}, J=6.8 \mathrm{~Hz}, 1 \mathrm{H}$, 4- $\left.\mathrm{H}_{\mathrm{B}}\right), 3.10(\mathrm{~m}, 2 \mathrm{H}, 1-\mathrm{H}), 2.99\left(\mathrm{~s}, 2 \mathrm{H}, 3-\mathrm{H}_{\mathrm{A}}\right), 2.97-2.86\left(\mathrm{~m}, 3 \mathrm{H}, 2-\mathrm{H} ; 5-\mathrm{H}_{\mathrm{A}}\right), 2.78(\mathrm{~s}, 1 \mathrm{H}, 3-\mathrm{H}), 2.60(\mathrm{t}, J=6.8 \mathrm{~Hz}$, $\left.1 \mathrm{H}, 5-\mathrm{H}_{\mathrm{B}}\right), 2.53(\mathrm{t}, J=5.3 \mathrm{~Hz}, 3 \mathrm{H}, 6-\mathrm{H}) .{ }^{1} \mathrm{H}-\mathrm{NMR}\left(400 \mathrm{MHz}\right.$, tetrachlorethan- $\left.d_{2}, 373 \mathrm{~K}\right): \delta(\mathrm{ppm})=8.20(\mathrm{t}$, $J=1.5 \mathrm{~Hz}, 1 \mathrm{H}, 19-\mathrm{H}), 7.87(\mathrm{~d}, J=2.6 \mathrm{~Hz}, 1 \mathrm{H}, 13-\mathrm{H}), 7.71(\mathrm{~d}, J=1.5 \mathrm{~Hz}, 2 \mathrm{H}, 21-\mathrm{H} ; 22-\mathrm{H}), 7.48(\mathrm{dd}, J=8.7,2.6 \mathrm{~Hz}$, $1 \mathrm{H}, 11-\mathrm{H}), 7.24(\mathrm{~d}, J=8.7 \mathrm{~Hz}, 1 \mathrm{H}, 10-\mathrm{H}), 3.62(\mathrm{~s}, 2 \mathrm{H}, 4-\mathrm{H}), 3.23(\mathrm{~d}, J=6.2 \mathrm{~Hz}, 2 \mathrm{H}, 1-\mathrm{H}), 3.18(\mathrm{~d}, J=6.1 \mathrm{~Hz}, 2 \mathrm{H}$, 2-H), $3.03(\mathrm{~s}, 3 \mathrm{H}, 6-\mathrm{H}), 2.88(\mathrm{~s}, 2 \mathrm{H}, 5-\mathrm{H}), 2.66(\mathrm{~s}, 3 \mathrm{H}, 3-\mathrm{H})$.

${ }^{13} \mathrm{C}-\mathrm{NMR}\left(101 \mathrm{MHz}, \mathrm{DMSO}-\mathrm{d}_{6}\right): \delta(\mathrm{ppm})=160.8(C=\mathrm{O}, \mathrm{C} 17), 153.1(C=\mathrm{O}, \mathrm{C} 8), 150.6(C=\mathrm{N}, \mathrm{C} 15), 147.9\left(C_{\mathrm{Ar}}, \mathrm{C}\right)$, $147.2\left(C_{\mathrm{Ar}}, \mathrm{C} 18\right), 134.9\left(C_{\mathrm{Ar}} \mathrm{H}, \mathrm{C} 11\right), 131.5\left(C_{\mathrm{Ar}}, \mathrm{C} 12\right), 131.4\left(C_{\mathrm{Ar}} \mathrm{H}, \mathrm{C} 21\right), 130.1\left(C_{\mathrm{Ar}} \mathrm{H}, \mathrm{C} 19\right), 129.6\left(C_{\mathrm{Ar}}, \mathrm{C} 23\right)$, $129.4\left(C_{\mathrm{Ar}} \mathrm{H}, \mathrm{C} 10\right), 128.5\left(C_{\mathrm{Ar}}, \mathrm{C} 14\right), 125.2\left(C_{\mathrm{Ar}} \mathrm{H}, \mathrm{C} 22\right), 125.0\left(C_{\mathrm{Ar}} \mathrm{H}, \mathrm{C} 13\right), 122.4\left(C_{\mathrm{Ar}}, \mathrm{C} 20\right), 47.7\left(\mathrm{CH}_{2}, \mathrm{C} 4\right), 47.0$ $\left(\mathrm{CH}_{2}, \mathrm{C} 1\right), 35.1\left(\mathrm{CH}_{2}, \mathrm{C} 5\right), 34.6\left(\mathrm{CH}_{3}, \mathrm{C} 3\right), 32.4\left(\mathrm{CH}_{3}, \mathrm{C} 6\right), 32.3\left(\mathrm{CH}_{2}, \mathrm{C} 2\right)$.

\section{chloro-2-(chloro-4-oxo-3,4-dihydroquinazolin-2-yl)phenyl 2-(((piperidin-2-ylmethyl)disulfaneyl) methyl)piperidine-carboxylate (SS06-PQ, 77)}

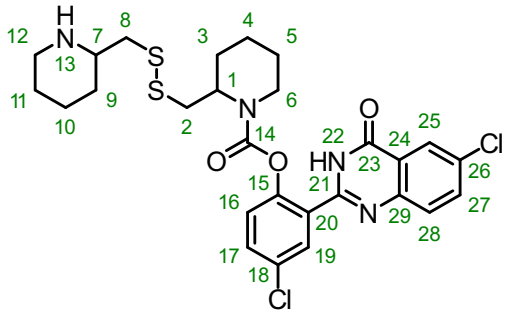

Prepared from $45(150 \mathrm{mg}, 0.45 \mathrm{mmol})$ and $55(138 \mathrm{mg}, 0.45 \mathrm{mmol})$ according to general protocol $\mathbf{G}$ to give 77 (90 mg, $0.15 \mathrm{mmol}, 33 \%)$ as a colourless solid after FCC (EtOAc/MeOH).

TLC $\mathrm{R}_{f}=0.15$ (DCM:MeOH, 19:1). HRMS (ESI $): \mathrm{C}_{27} \mathrm{H}_{31} \mathrm{Cl}_{2} \mathrm{~N}_{4} \mathrm{O}_{3} \mathrm{~S}_{2}{ }^{+}[\mathrm{M}+\mathrm{H}]^{+}$: calc. 593.12091, found 593.12024.

${ }^{1} \mathrm{H}-\mathrm{NMR}\left(400 \mathrm{MHz}\right.$, tetrachlorethan $\left.d_{2}, 373 \mathrm{~K}\right): \delta(\mathrm{ppm})=8.27-8.16(\mathrm{~m}, 1 \mathrm{H}, 25-\mathrm{H}), 7.91(\mathrm{t}, \mathrm{J}=2.8 \mathrm{~Hz}, 1 \mathrm{H}, 19-\mathrm{H})$, $7.75-7.66(\mathrm{~m}, 2 \mathrm{H}, 27-\mathrm{H} ; 28-\mathrm{H}), 7.52-7.44(\mathrm{~m}, 1 \mathrm{H}, 17-\mathrm{H}), 7.38-7.21(\mathrm{~m}, 1 \mathrm{H}, 16-\mathrm{H}), 4.57(\mathrm{~s}, 1 \mathrm{H}, \mathrm{NH}, 13-\mathrm{H}), 4.05$ $(\mathrm{d}, J=14.5 \mathrm{~Hz}, 1 \mathrm{H}, 1-\mathrm{H}), 3.65-3.42(\mathrm{~m}, 1 \mathrm{H}), 3.39-3.27(\mathrm{~m}, 2 \mathrm{H}), 3.20(\mathrm{dd}, J=13.5,7.0 \mathrm{~Hz}, 1 \mathrm{H}), 3.11(\mathrm{~s}, 1 \mathrm{H})$, $3.01(\mathrm{dd}, J=14.0,6.7 \mathrm{~Hz}, 1 \mathrm{H}), 2.96-2.68(\mathrm{~m}, 3 \mathrm{H}), 2.09-1.90(\mathrm{~m}, 2 \mathrm{H}), 1.90-1.82(\mathrm{~m}, 2 \mathrm{H}), 1.81-1.73(\mathrm{~m}, 2 \mathrm{H})$, 1.65 (t, $J=9.7 \mathrm{~Hz}, 2 \mathrm{H}), 1.46$ (dd, $J=22.1,10.9 \mathrm{~Hz}, 4 \mathrm{H})$.

${ }^{13} \mathrm{C}$-NMR $\left(400 \mathrm{MHz}\right.$, tetrachlorethan- $\left.d_{2}, 373 \mathrm{~K}\right): \delta(\mathrm{ppm})=160.5(C=\mathrm{O}, \mathrm{C} 23), 152.5(C=\mathrm{O}, \mathrm{C} 14), 149.4(C=\mathrm{N}, \mathrm{C} 21)$, $147.9\left(C_{\mathrm{Ar}}, \mathrm{C} 15\right), 147.6\left(C_{\mathrm{Ar}}, \mathrm{C} 24\right), 135.0\left(C_{\mathrm{Ar}} \mathrm{H}, \mathrm{C} 28\right), 133.2\left(C_{\mathrm{Ar}}, \mathrm{C} 29\right), 131.9\left(C_{\mathrm{Ar}} \mathrm{H}, \mathrm{C} 17\right), 131.7\left(C_{\mathrm{Ar}}, \mathrm{C} 18\right), 130.4$ $\left(C_{\mathrm{Ar}} \mathrm{H}, \mathrm{C} 19\right), 129.7\left(C_{\mathrm{Ar}} \mathrm{H}, \mathrm{C} 27\right), 128.2\left(C_{\mathrm{Ar}}, \mathrm{C} 20\right), 126.0\left(C_{\mathrm{Ar}} \mathrm{H}, \mathrm{C} 25\right), 124.9\left(C_{\mathrm{Ar}} \mathrm{H}, \mathrm{C} 16\right), 122.5\left(C_{\mathrm{Ar}}, \mathrm{C} 26\right), 56.8$ $(\mathrm{CH}, \mathrm{C} 7), 55.7(\mathrm{CH}, \mathrm{C} 1), 45.3\left(\mathrm{CH}_{2}\right), 41.2\left(\mathrm{CH}_{2}\right), 40.7\left(\mathrm{CH}_{2}\right), 28.2\left(\mathrm{CH}_{2}\right), 27.5\left(\mathrm{CH}_{2}\right), 25.0\left(\mathrm{CH}_{2}\right), 22.5\left(\mathrm{CH}_{2}\right)$, $22.0\left(\mathrm{CH}_{2}\right), 18.7\left(\mathrm{CH}_{2}\right)$. 


\section{MF-Series}

trans-3'-methoxy-3-oxo-3H-spiro[isobenzofuran-1,9'-xanthen]-6'-yl hexahydro-[1,2]dithiino [4,5-b]pyridine-1 $(2 H)$-carboxylate (SS66T-MF, 78)

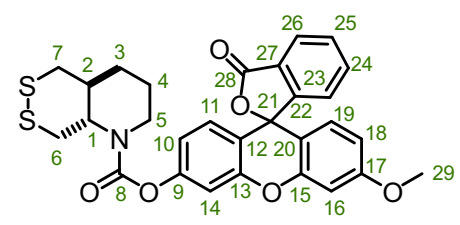

Prepared from $8(21.2 \mathrm{mg}, 0.100 \mathrm{mmol})$ and $58(38.1 \mathrm{mg}, 0.110 \mathrm{mmol})$ according to general protocol G to give 78 (27.1 $\mathrm{mg}, 0.049 \mathrm{mmol}, 49 \%$ ) as a colourless solid after FCC (isohexane/EtOAc).

TLC $\mathrm{R}_{f}=0.33$ (isohexane:EtOAc, 3:1). HRMS $\left(E S{ }^{+}\right) \mathrm{C}_{29} \mathrm{H}_{26} \mathrm{NO}_{6} \mathrm{~S}_{2}{ }^{+}[\mathrm{M}+\mathrm{H}]^{+}$: calc. 548.11961, found 548.11973.

${ }^{1} \mathrm{H}-N M R\left(500 \mathrm{MHz}, \mathrm{CDCl}_{3}\right): \delta(\mathrm{ppm})=8.02(\mathrm{~d}, J=7.1 \mathrm{~Hz}, 1 \mathrm{H}, 26-\mathrm{H}), 7.67(\mathrm{tt}, J=7.5,1.4 \mathrm{~Hz}, 1 \mathrm{H}, 24-\mathrm{H}), 7.62(\mathrm{t}$, $J=7.3 \mathrm{~Hz}, 1 \mathrm{H}, 25-\mathrm{H}), 7.15(\mathrm{~d}, J=7.4 \mathrm{~Hz}, 1 \mathrm{H}, 23-\mathrm{H}), 7.09(\mathrm{dd}, J=3.7,1.7 \mathrm{~Hz}, 1 \mathrm{H}, 14-\mathrm{H}), 6.84-6.77(\mathrm{~m}, 2 \mathrm{H}, 10-\mathrm{H}$; $11-\mathrm{H}), 6.76(\mathrm{~d}, J=2.5 \mathrm{~Hz}, 1 \mathrm{H}, 16-\mathrm{H}), 6.70(\mathrm{~d}, J=8.8 \mathrm{~Hz}, 1 \mathrm{H}, 19-\mathrm{H}), 6.62(\mathrm{dd}, J=8.8,2.5 \mathrm{~Hz}, 1 \mathrm{H}, 18-\mathrm{H}), 3.94-$ $3.84\left(\mathrm{~m}, 1 \mathrm{H}, 5-\mathrm{H}_{\mathrm{A}}\right), 3.84(\mathrm{~s}, 3 \mathrm{H}, 29-\mathrm{H}), 3.80\left(\mathrm{dd}, J=10.1,3.4 \mathrm{~Hz}, 1 \mathrm{H}, 6-\mathrm{H}_{\mathrm{A}}\right), 3.35(\mathrm{~d}, J=15.2 \mathrm{~Hz}, 1 \mathrm{H}, 1-\mathrm{H}), 3.20-$ $3.06\left(\mathrm{~m}, 2 \mathrm{H}, 5-\mathrm{H}_{\mathrm{B}}, 6-\mathrm{H}_{\mathrm{B}}\right), 2.94\left(\mathrm{dd}, J=13.5,10.8 \mathrm{~Hz}, 1 \mathrm{H}, 7-\mathrm{H}_{\mathrm{A}}\right), 2.81\left(\mathrm{dd}, J=13.5,2.7 \mathrm{~Hz}, 1 \mathrm{H}, 7-\mathrm{H}_{\mathrm{B}}\right), 2.23-2.09$ $(\mathrm{m}, 1 \mathrm{H}, 2-\mathrm{H}), 1.96\left(\mathrm{dt}, J=19.0,8.5 \mathrm{~Hz}, 1 \mathrm{H}, 3-\mathrm{H}_{\mathrm{A}}\right), 1.84-1.72\left(\mathrm{~m}, 1 \mathrm{H}, 4-\mathrm{H}_{\mathrm{A}}\right), 1.72-1.60\left(\mathrm{~m}, 1 \mathrm{H}, 3-\mathrm{H}_{\mathrm{B}}\right), 1.48-$ $1.36\left(\mathrm{~m}, 1 \mathrm{H}, 4-\mathrm{H}_{\mathrm{B}}\right)$.

${ }^{13} \mathrm{C}-N M R\left(101 \mathrm{MHz}, \mathrm{CDCl}_{3}\right): \delta(\mathrm{ppm})=169.5(C=\mathrm{O}, \mathrm{C} 28), 161.5\left(C_{\mathrm{Ar}}, \mathrm{C} 17\right), 153.4(C=\mathrm{O}, \mathrm{C} 8), 153.2\left(C_{\mathrm{Ar}}, \mathrm{C} 27\right)$, $152.6\left(C_{\mathrm{Ar}}, C 13\right), 152.4\left(C_{\mathrm{Ar}}, \mathrm{C} 20\right), 152.0\left(C_{\mathrm{Ar}}, \mathrm{C} 15\right), 135.2\left(C_{\mathrm{Ar}} \mathrm{H}, \mathrm{C} 23\right), 130.0\left(C_{\mathrm{Ar}} \mathrm{H}, \mathrm{C} 25\right), 129.2\left(C_{\mathrm{Ar}} \mathrm{H}, \mathrm{C} 10\right)$, $129.1\left(C_{\mathrm{Ar}} \mathrm{H}, \mathrm{C} 19\right), 126.6\left(C_{\mathrm{Ar}}, \mathrm{C} 22\right), 125.2\left(C_{\mathrm{Ar}} \mathrm{H}, \mathrm{C} 26\right), 124.2\left(C_{\mathrm{Ar}} \mathrm{H}, \mathrm{C} 23\right), 117.6\left(C_{\mathrm{Ar}} \mathrm{H}, \mathrm{C} 11\right), 116.4\left(C_{\mathrm{Ar}}, \mathrm{C} 12\right)$, $112.0\left(C_{\mathrm{Ar}} \mathrm{H}, \mathrm{C} 18\right), 111.0\left(C_{\mathrm{Ar}}, \mathrm{C} 9\right), 110.4\left(C_{\mathrm{Ar}} \mathrm{H}, \mathrm{C} 14\right), 101.0\left(C_{\mathrm{Ar}} \mathrm{H}, \mathrm{C} 16\right), 82.7\left(C_{\text {spiro }}, \mathrm{C} 21\right), 63.1(\mathrm{CH}, \mathrm{C} 1), 55.7$ $\left(\mathrm{CH}_{3}, \mathrm{C} 29\right), 41.1\left(\mathrm{CH}_{2}, \mathrm{C} 6\right), 40.7\left(\mathrm{CH}_{2}, \mathrm{C} 7\right), 37.0\left(\mathrm{CH}_{2}, \mathrm{C} 5\right), 34.3(\mathrm{CH}, \mathrm{C} 2), 27.2\left(\mathrm{CH}_{2}, \mathrm{C} 3\right), 23.1\left(\mathrm{CH}_{2}, \mathrm{C} 4\right) .22 .8$, 22.5, 20.6.

\section{cis-3'-methoxy-3-oxo-3H-spiro[isobenzofuran-1,9'-xanthen]-6'-yl hexahydro-[1,2]dithiino} [4,5-b]pyridine-1(2H)-carboxylate (SS66C-MF, 79)

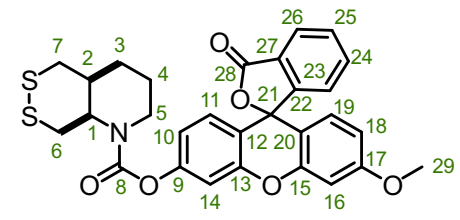

Prepared from $18(14.0 \mathrm{mg}, 0.066 \mathrm{mmol})$ and $\mathbf{5 8}(25.1 \mathrm{mg}, 0.073 \mathrm{mmol})$ according to general protocol $\mathbf{G}$ to give $79(11.0 \mathrm{mg}, 0.0 .02 \mathrm{mmol}, 30 \%)$ as a colourless solid after FCC (isohexane/EtOAc).

TLC $\mathrm{R}_{f}=0.34$ (isohexane:EtOAc, 3:1). HRMS (ESI $\left.{ }^{+}\right) \mathrm{C}_{29} \mathrm{H}_{26} \mathrm{NO}_{6} \mathrm{~S}_{2}{ }^{+}[\mathrm{M}+\mathrm{H}]^{+}$: calc. 548.11961, found 548.11996.

${ }^{1} \mathrm{H}-\mathrm{NMR}\left(500 \mathrm{MHz}, \mathrm{CDCl}_{3}\right): \delta(\mathrm{ppm})=8.02(\mathrm{~d}, J=7.4 \mathrm{~Hz}, 1 \mathrm{H}, 26-\mathrm{H}), 7.67(\mathrm{t}, J=7.3 \mathrm{~Hz}, 1 \mathrm{H}, 24-\mathrm{H}), 7.62(\mathrm{t}$, $J=7.3 \mathrm{~Hz}, 1 \mathrm{H}, 25-\mathrm{H}), 7.16(\mathrm{~d}, J=7.5 \mathrm{H}, 1 \mathrm{H}, 23-\mathrm{H}), 7.12-7.01(\mathrm{~m}, 1 \mathrm{H}, 14-\mathrm{H}), 6.82-6.77(\mathrm{~m}, 2 \mathrm{H}, 10-\mathrm{H} ; 11-\mathrm{H})$, $6.76(\mathrm{~d}, J=2.4 \mathrm{~Hz}, 1 \mathrm{H}, 16-\mathrm{H}), 6.70(\mathrm{~d}, J=8.8 \mathrm{~Hz}, 1 \mathrm{H}, 19-\mathrm{H}), 6.62(\mathrm{dd}, J=8.8,2.4 \mathrm{~Hz}, 1 \mathrm{H}, 18-\mathrm{H}), 4.56(\mathrm{t}$, $J=10.9 \mathrm{~Hz}, 1 \mathrm{H}, 1-\mathrm{H}), 4.14\left(\mathrm{t}, J=12.5 \mathrm{~Hz}, 1 \mathrm{H}, 5-\mathrm{H}_{\mathrm{A}}\right), 3.84(\mathrm{~s}, 3 \mathrm{H}, 29-\mathrm{H}), 3.62-3.42\left(\mathrm{~m}, 2 \mathrm{H}, 6-\mathrm{H}_{\mathrm{A}} ; 7-\mathrm{H}_{\mathrm{A}}\right), 2.97(\mathrm{dt}$, $\left.J=44.8,12.0 \mathrm{~Hz}, 1 \mathrm{H}, 5-\mathrm{H}_{\mathrm{B}}\right), 2.83\left(\mathrm{t}, J=15.1 \mathrm{~Hz}, 1 \mathrm{H}, 7-\mathrm{H}_{\mathrm{B}}\right.$ ), 2.51 (ddt, $\left.J=10.9,7.0,4.1 \mathrm{~Hz}, 1 \mathrm{H}, 2-\mathrm{H}\right), 2.39-2.21$ $\left(\mathrm{m}, 2 \mathrm{H}, 3-\mathrm{H}_{\mathrm{A}} ; 6-\mathrm{H}_{\mathrm{B}}\right), 1.86\left(\mathrm{~d}, J=13.4 \mathrm{~Hz}, 1 \mathrm{H}, 4-\mathrm{H}_{\mathrm{A}}\right), 1.74-1.59\left(\mathrm{~m}, 1 \mathrm{H}, 3-\mathrm{H}_{\mathrm{B}}\right), 1.52\left(\mathrm{~d}, J=11.9 \mathrm{~Hz}, 1 \mathrm{H}, 4-\mathrm{H}_{\mathrm{B}}\right)$.

${ }^{13}$ C-NMR $\left(101 \mathrm{MHz}, \mathrm{CDCl}_{3}\right): \delta(\mathrm{ppm})=169.5(C=\mathrm{O}, \mathrm{C} 28), 161.5\left(C_{\mathrm{Ar}}, \mathrm{C} 17\right), 153.2(C=\mathrm{O}, \mathrm{C}), 153.0\left(C_{\mathrm{Ar}}, \mathrm{C} 27\right)$, $152.7\left(C_{A r}, C 13\right), 152.4\left(C_{A r}, C 20\right), 152.0\left(C_{A r}, C 15\right), 135.2\left(C_{A r} H, C 23\right), 130.0\left(C_{A r} H, C 25\right), 129.2\left(C_{A r} H, C 10\right)$, $129.0\left(C_{\mathrm{Ar}} \mathrm{H}, \mathrm{C} 19\right), 126.6\left(C_{\mathrm{Ar}}, \mathrm{C} 22\right), 125.2\left(C_{\mathrm{Ar}} \mathrm{H}, \mathrm{C} 26\right), 124.2\left(C_{\mathrm{Ar}} \mathrm{H}, \mathrm{C} 23\right), 117.6\left(C_{\mathrm{Ar}} \mathrm{H}, \mathrm{C} 11\right), 116.3\left(C_{\mathrm{Ar}}, \mathrm{C} 12\right)$, $112.0\left(C_{\mathrm{Ar}} \mathrm{H}, \mathrm{C} 18\right), 111.0\left(C_{\mathrm{Ar}}, \mathrm{C} 9\right), 110.4\left(C_{\mathrm{Ar}} \mathrm{H}, \mathrm{C} 14\right), 101.0\left(C_{\mathrm{Ar}} \mathrm{H}, \mathrm{C} 16\right), 82.7\left(C_{\text {spiro }}, \mathrm{C} 21\right), 55.7\left(C_{3}, \mathrm{C} 29\right), 54.1$ ( $\mathrm{CH}, \mathrm{C} 1$; minor $), 53.3(\mathrm{CH}, \mathrm{C} 1$; major $), 42.3\left(\mathrm{CH}_{2}, \mathrm{C} 6\right.$; major $), 42.2\left(\mathrm{CH}_{2}, \mathrm{C} 6\right.$; minor $), 40.7\left(\mathrm{CH}_{2}, \mathrm{C} 7 ;\right.$ major $), 40.2$ $\left(\mathrm{CH}_{2}, \mathrm{C} 7\right.$; minor), $36.2\left(\mathrm{CH}_{2}, \mathrm{C}\right.$; minor), $35.9\left(\mathrm{CH}_{2}, \mathrm{C}\right.$; major), 30.1 ( $\mathrm{CH}, \mathrm{C} 2$; minor), 29.4 ( $\mathrm{CH}, \mathrm{C} 2$; major $), 25.5$ $\left(\mathrm{CH}_{2}, \mathrm{C} 3\right.$; major $), 25.0\left(\mathrm{CH}_{2}, \mathrm{C} 3\right.$; minor $), 23.6\left(\mathrm{CH}_{2}, \mathrm{C} 2\right)$.

cis-3'-methoxy-3-oxo-3H-spiro[isobenzofuran-1,9'-xanthen]-6'-yl hexahydrofuro[3,4-b]pyridine-1(2H)-carboxylate (O56-MF, 80)

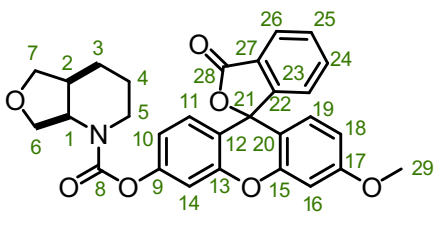


Prepared from $19(65 \mathrm{mg}, 0.40 \mathrm{mmol})$ and $58(208 \mathrm{mg}, 0.601 \mathrm{mmol})$ according to general protocol G to give 80 $(158 \mathrm{mg}, 0.316 \mathrm{mmol}, 79 \%)$ as a colourless solid after FCC $\left(\mathrm{Et}_{2} \mathrm{O}\right)$.

TLC $\mathrm{R}_{f}=0.29$ (isohexane:EtOAc, 1:1). HRMS (ESI): $\mathrm{C}_{29} \mathrm{H}_{26} \mathrm{NO}_{7}{ }^{+}[\mathrm{M}+\mathrm{H}]^{+}$: calc. 500.17038, found 500.17059.

${ }^{1} \mathrm{H}-N M R\left(400 \mathrm{MHz}, \mathrm{CDCl}_{3}\right): \delta(\mathrm{ppm})=8.02(\mathrm{~d}, J=7.6 \mathrm{~Hz}, 1 \mathrm{H}, 26-\mathrm{H}), 7.67(\mathrm{tt}, J=7.5,1.2 \mathrm{~Hz}, 1 \mathrm{H}, 24-\mathrm{H}), 7.62(\mathrm{td}$, $J=7.3 \mathrm{~Hz}, 1 \mathrm{H}, 25-\mathrm{H}), 7.16(\mathrm{dt}, J=7.5,0.9 \mathrm{~Hz}, 1 \mathrm{H}, 23-\mathrm{H}), 7.09(\mathrm{~s}, 1 \mathrm{H}, 14-\mathrm{H}), 6.84-6.78(\mathrm{~m}, 2 \mathrm{H}, 10-\mathrm{H} ; 11-\mathrm{H})$, $6.76(\mathrm{~d}, J=2.4 \mathrm{~Hz}, 1 \mathrm{H}, 16-\mathrm{H}), 6.70(\mathrm{~d}, J=8.8 \mathrm{~Hz}, 1 \mathrm{H}, 19-\mathrm{H}), 6.62(\mathrm{dd}, J=8.8,2.5 \mathrm{~Hz}, 1 \mathrm{H}, 18-\mathrm{H}), 4.85(\mathrm{~s}, 1 \mathrm{H}, 1-\mathrm{H})$, $4.12\left(\mathrm{~s}, 1 \mathrm{H}, 5-\mathrm{H}_{\mathrm{A}}\right), 4.03\left(\mathrm{t}, \mathrm{J}=8.4 \mathrm{~Hz}, 1 \mathrm{H}, 6-\mathrm{H}_{\mathrm{A}}\right), 3.92\left(\mathrm{~s}, 1 \mathrm{H}, 7-\mathrm{H}_{\mathrm{A}}\right), 3.84(\mathrm{~s}, 3 \mathrm{H}, 29-\mathrm{H}), 3.72(\mathrm{~d}, J=8.6 \mathrm{~Hz}, 2 \mathrm{H}$, $\left.6-\mathrm{H}_{B} ; 7-\mathrm{H}_{\mathrm{B}}\right), 3.13-2.83\left(\mathrm{~m}, 1 \mathrm{H}, 5-\mathrm{H}_{\mathrm{B}}\right), 2.35-2.16(\mathrm{~m}, 1 \mathrm{H}, 2-\mathrm{H}), 1.93-1.74\left(\mathrm{~m}, 2 \mathrm{H}, 3-\mathrm{H}_{\mathrm{A}} ; 4-\mathrm{H}_{\mathrm{A}}\right), 1.57-1.49(\mathrm{~m}$, $\left.2 \mathrm{H}, 3-\mathrm{H}_{\mathrm{B}} ; 4-\mathrm{H}_{\mathrm{B}}\right)$.

${ }^{13}$ C-NMR $\left(101 \mathrm{MHz}, \mathrm{CDCl}_{3}\right): \delta(\mathrm{ppm})=169.5(C=\mathrm{O}, \mathrm{C} 28), 161.6\left(C_{\mathrm{Ar}}, \mathrm{C} 17\right), 153.9(C=\mathrm{O}, \mathrm{C} 8), 153.2\left(C_{\mathrm{Ar}}, \mathrm{C} 27\right)$, $152.7\left(C_{\mathrm{Ar}}, \mathrm{C} 13\right), 152.5\left(C_{\mathrm{Ar}}, \mathrm{C} 20\right), 152.0\left(C_{\mathrm{Ar}}, \mathrm{C} 15\right), 135.2\left(C_{\mathrm{Ar}} \mathrm{H}, \mathrm{C} 23\right), 130.0\left(C_{\mathrm{Ar}} \mathrm{H}, \mathrm{C} 25\right), 129.2\left(C_{\mathrm{Ar}} \mathrm{H}, \mathrm{C} 10\right)$, $129.0\left(C_{\mathrm{Ar}} \mathrm{H}, \mathrm{C} 19\right), 126.6\left(C_{\mathrm{Ar}}, \mathrm{C} 22\right), 125.2\left(C_{\mathrm{Ar}} \mathrm{H}, \mathrm{C} 26\right), 124.2\left(C_{\mathrm{Ar}} \mathrm{H}, \mathrm{C} 23\right), 117.6\left(C_{\mathrm{Ar}} \mathrm{H}, \mathrm{C} 11\right), 116.6\left(C_{\mathrm{Ar}}, \mathrm{C} 12\right)$, $112.0\left(C_{\mathrm{Ar}} \mathrm{H}, \mathrm{C} 18\right), 111.1\left(C_{\mathrm{Ar}}, \mathrm{C} 9\right), 110.5\left(C_{\mathrm{Ar}} \mathrm{H}, \mathrm{C} 14\right), 101.0\left(C_{\mathrm{Ar}} \mathrm{H}, \mathrm{C} 16\right), 82.7\left(C_{\text {spiro }}, \mathrm{C} 21\right), 73.4\left(\mathrm{CH}_{2}, \mathrm{C} 7\right), 66.3$ $\left(\mathrm{CH}_{2}, \mathrm{C} 6\right), 55.7\left(\mathrm{CH}_{3}, \mathrm{C} 29\right), 54.4(\mathrm{CH}, \mathrm{C} 1), 41.0\left(\mathrm{CH}_{2}, \mathrm{C} 5\right), 36.1(\mathrm{CH}, \mathrm{C} 2), 25.1\left(\mathrm{CH}_{2}, \mathrm{C} 3\right), 23.6\left(\mathrm{CH}_{2}, \mathrm{C} 4\right)$.

\section{3'-methoxy-3-oxo-3H-spiro[isobenzofuran-1,9'-xanthen]-6'-yl ((S)-1,2-dithian-4- yl)(methyl)carbamate (SS60-MF, 81)}

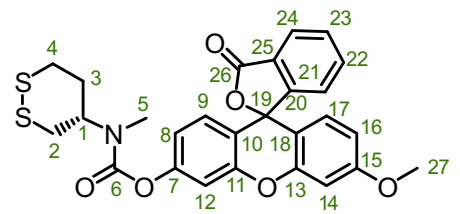

Prepared from $25(74 \mathrm{mg}, 0.40 \mathrm{mmol})$ and $58(213 \mathrm{mg}, 0.62 \mathrm{mmol})$ according to general protocol G to give 81 (133 $\mathrm{mg}, 0.26 \mathrm{mmol}, 65 \%)$ as a colourless solid after $\mathrm{FCC}\left(\mathrm{Et}_{2} \mathrm{O}\right)$.

TLC $\mathrm{R}_{f}=0.35$ (isohexane:EtOAc, 2:1). HRMS (ESI ${ }^{+}$): $\mathrm{C}_{27} \mathrm{H}_{24} \mathrm{NO}_{6} \mathrm{~S}_{2}{ }^{+}[\mathrm{M}+\mathrm{H}]^{+}$: calc. 522.10396, found 482.10438.

Individual rotamers were observed by NMR spectroscopy at $298 \mathrm{~K}$ (ratio ca. 1.5:1). The following data refers to all signals observed at $298 \mathrm{~K}$.

${ }^{1} \mathrm{H}-N M R\left(400 \mathrm{MHz}, \mathrm{CDCl}_{3}\right): \delta(\mathrm{ppm})=8.02(\mathrm{dd}, J=8.0,1.1 \mathrm{~Hz}, 1 \mathrm{H}, 24-\mathrm{H}), 7.67(\mathrm{td}, J=7.4,1.4 \mathrm{~Hz}, 1 \mathrm{H}, 22-\mathrm{H})$, $7.62(\mathrm{td}, J=7.4,1.2 \mathrm{~Hz}, 1 \mathrm{H}, 23-\mathrm{H}), 7.16(\mathrm{dt}, J=7.7,1.0 \mathrm{~Hz}, 1 \mathrm{H}, 21-\mathrm{H}), 7.09(\mathrm{~d}, J=1.8 \mathrm{~Hz}, 1 \mathrm{H}, 12-\mathrm{H}), 6.83-6.78$ $(\mathrm{m}, 2 \mathrm{H}, 8-\mathrm{H} ; 9-\mathrm{H}), 6.76(\mathrm{~d}, J=2.4 \mathrm{~Hz}, 1 \mathrm{H}, 14-\mathrm{H}), 6.70(\mathrm{~d}, J=8.8 \mathrm{~Hz}, 1 \mathrm{H}, 17-\mathrm{H}), 6.62(\mathrm{dd}, J=8.8,2.5 \mathrm{~Hz}, 1 \mathrm{H}$, $16-\mathrm{H}), 4.33(\mathrm{t}, J=11.3 \mathrm{~Hz}, 1 \mathrm{H}, 1-\mathrm{H}), 3.84(\mathrm{~s}, 3 \mathrm{H}, 27-\mathrm{H}), 3.24(\mathrm{t}, J=13.1 \mathrm{~Hz}, 1 \mathrm{H}, 3-\mathrm{H}), 3.14(\mathrm{t}, J=12.3 \mathrm{~Hz}, 1 \mathrm{H}$, 2-H), $3.07-2.97\left(\mathrm{~m}, 3 \mathrm{H}, 3\right.$ '- H; 5-H), $2.94(\mathrm{~s}, 1 \mathrm{H}, 5$ '- $), 2.80\left(\mathrm{t}, J=16.4 \mathrm{~Hz}, 1 \mathrm{H}, 2^{\prime}-\mathrm{H}\right), 2.23(\mathrm{dd}, J=25.0,14.0 \mathrm{~Hz}$, $1 \mathrm{H}, 4-\mathrm{H}), 2.09\left(\mathrm{dd}, J=11.7,10.6 \mathrm{~Hz}, 1 \mathrm{H}, 4{ }^{\prime}-\mathrm{H}\right) .{ }^{13} \mathrm{C}-\mathrm{NMR}\left(101 \mathrm{MHz}, \mathrm{CDCl}_{3}\right): \delta(\mathrm{ppm})=169.5(C=\mathrm{O}, \mathrm{C} 26), 161.6$ $\left(C_{\mathrm{Ar}}, \mathrm{C} 15\right), 153.7(C=\mathrm{O}, \mathrm{C}), 153.2\left(C_{\mathrm{Ar}}, \mathrm{C} 25\right), 152.7\left(C_{\mathrm{Ar}}, C 11\right), 152.6\left(C_{\mathrm{Ar}}, \mathrm{C} 11^{\prime}\right), 152.4\left(C_{\mathrm{Ar}}, \mathrm{C} 18\right), 152.0\left(C_{\mathrm{Ar}}\right.$, C13), $135.2\left(C_{\mathrm{Ar}} \mathrm{H}, \mathrm{C} 21\right), 130.0\left(C_{\mathrm{Ar}} \mathrm{H}, \mathrm{C} 23\right), 129.2\left(C_{\mathrm{Ar}} \mathrm{H}, \mathrm{C}\right), 129.0\left(C_{\mathrm{Ar}} \mathrm{H}, \mathrm{C} 17\right), 126.6\left(C_{\mathrm{Ar}}, \mathrm{C} 20\right), 125.2\left(C_{\mathrm{Ar}} \mathrm{H}\right.$, C24), $124.2\left(C_{A r} H, C 21\right), 117.6\left(C_{A r} H, C 9\right), 116.6\left(C_{A r}, C 10\right), 112.0\left(C_{A r} H, C 16\right), 111.1\left(C_{A r}, C 7\right), 110.5\left(C_{A r} H, C 12\right)$, $101.0\left(C_{\mathrm{Ar}} \mathrm{H}, \mathrm{C} 14\right), 82.7\left(C_{\text {spiro }}, \mathrm{C} 19\right), 56.1(\mathrm{CH}, \mathrm{C} 1), 55.7\left(\mathrm{CH}_{3}, \mathrm{C} 27\right), 36.3\left(\mathrm{CH}_{2}, \mathrm{C} 3\right), 36.2\left(\mathrm{CH}_{2}, \mathrm{C}^{\prime}\right), 36.1\left(\mathrm{CH}_{2}\right.$, $\mathrm{C} 2), 35.7\left(\mathrm{CH}_{2}, \mathrm{C} 2\right), 33.2\left(\mathrm{CH}_{2}, \mathrm{C} 4\right), 32.5\left(\mathrm{CH}_{2}, \mathrm{C}^{\prime}\right), 30.1\left(\mathrm{CH}_{3}, \mathrm{C} 5\right)$.

\section{3'-methoxy-3-oxo-3H-spiro[isobenzofuran-1,9'-xanthen]-6'-yl (1,2-dithiolan-4-yl) (methyl)carbamate (SS50-MF, 82)}

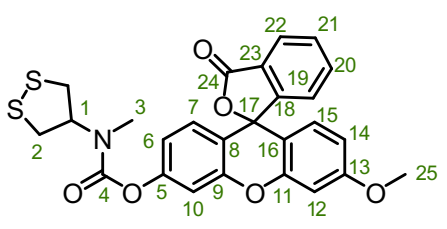

Prepared from $52(75.0 \mathrm{mg}, 0.319 \mathrm{mmol})$ and $\mathbf{5 8}(221.0 \mathrm{mg}, 0.637 \mathrm{mmol})$ according to general protocol $\mathbf{G}$ to give $82(32.0 \mathrm{mg}, 0.063 \mathrm{mmol}, 20 \%)$ as a colourless solid after FCC (aluminium oxide, isohexane/EtOAc).

TLC $R_{f}=0.43$ (silica, isohexane:EtOAc, 2:1); $R_{f}=0.40$ (aluminium oxide, isohexane:EtOAc, 5:2).

HRMS $\left(E S I^{+}\right) \mathrm{C}_{26} \mathrm{H}_{22} \mathrm{NO}_{6} \mathrm{~S}_{2}{ }^{+}[\mathrm{M}+\mathrm{H}]^{+}$: calc. 508.08831, found 508.08862.

Individual rotamers were observed by NMR spectroscopy at $298 \mathrm{~K}$ (ratio ca. 1.2:1). The following data refers to all signals observed at $298 \mathrm{~K}$.

${ }^{1} \mathrm{H}-\mathrm{NMR}\left(400 \mathrm{MHz}, \mathrm{CDCl}_{3}\right): \delta(\mathrm{ppm})=8.03(\mathrm{dt}, J=7.3,1.2 \mathrm{~Hz}, 1 \mathrm{H}, 22-\mathrm{H}), 7.67(\mathrm{td}, J=7.4,1.4 \mathrm{~Hz}, 1 \mathrm{H}, 20-\mathrm{H}), 7.62$ $(\mathrm{td}, J=7.4,1.2 \mathrm{~Hz}, 1 \mathrm{H}, 21-\mathrm{H}), 7.16(\mathrm{~d}, J=7.7 \mathrm{~Hz}, 1 \mathrm{H}, 19-\mathrm{H}), 7.10(\mathrm{~d}, J=1.8 \mathrm{~Hz}, 1 \mathrm{H}, 10-\mathrm{H}), 6.84-6.79(\mathrm{~m}, 2 \mathrm{H}$, $6-\mathrm{H} ; 7-\mathrm{H}), 6.77$ (d, J = 2.4 Hz, 1H, 12-H), $6.70(\mathrm{~d}, J=8.9 \mathrm{~Hz}, 1 \mathrm{H}, 15-\mathrm{H}), 6.63(\mathrm{dd}, J=8.9,2.5 \mathrm{~Hz}, 1 \mathrm{H}, 14-\mathrm{H}), 5.27$ 
(ddd, $J=13.3,7.8,5.6 \mathrm{~Hz}, 1 \mathrm{H}, 1-\mathrm{H}), 3.84(\mathrm{~s}, 3 \mathrm{H}, 25-\mathrm{H}), 3.51-3.33\left(\mathrm{~m}, 2 \mathrm{H}, 2-\mathrm{H}_{\mathrm{A}}\right), 3.24-3.11\left(\mathrm{~m}, 2 \mathrm{H}, 2-\mathrm{H}_{\mathrm{B}}\right), 3.09$ (s, $2 \mathrm{H}, 3-\mathrm{H}$; major), 3.00 (s, $1 \mathrm{H}, 3-\mathrm{H}$; minor).

${ }^{13}$ C-NMR $\left(101 \mathrm{MHz}, \mathrm{CDCl}_{3}\right): \delta(\mathrm{ppm})=169.5(C=\mathrm{O}, \mathrm{C} 24), 161.6\left(C_{\mathrm{Ar}}, \mathrm{C} 13\right), 153.2(C=\mathrm{O}, \mathrm{C} 4), 153.1\left(C_{\mathrm{Ar}}, \mathrm{C} 23\right)$, 152.6 ( $\left.C_{\mathrm{Ar}}, C 9\right), 152.4\left(C_{\mathrm{Ar}}, \mathrm{C} 16\right), 152.0\left(C_{\mathrm{Ar}}, \mathrm{C} 11\right), 135.2\left(C_{\mathrm{Ar}} \mathrm{H}, \mathrm{C} 19\right), 130.0\left(C_{\mathrm{Ar}} \mathrm{H}, \mathrm{C} 21\right), 129.2\left(C_{\mathrm{Ar}} \mathrm{H}, \mathrm{C} 6\right), 129.1$ $\left(C_{\mathrm{Ar}} \mathrm{H}, \mathrm{C} 15\right), 126.7\left(C_{\mathrm{Ar}}, \mathrm{C} 18\right), 125.2\left(C_{\mathrm{Ar}} \mathrm{H}, \mathrm{C} 22\right), 124.2\left(C_{\mathrm{Ar}} \mathrm{H}, \mathrm{C} 19\right), 117.6\left(C_{\mathrm{Ar}} \mathrm{H}, \mathrm{C}\right), 116.6\left(C_{\mathrm{Ar}}, \mathrm{C}\right), 112.0$ $\left(C_{\mathrm{Ar}} \mathrm{H}, \mathrm{C} 14\right), 111.1\left(C_{\mathrm{Ar}}, \mathrm{C} 5\right), 110.4\left(\mathrm{C}_{\mathrm{Ar}} \mathrm{H}, \mathrm{C} 10\right), 101.0\left(\mathrm{C}_{\mathrm{Ar}} \mathrm{H}, \mathrm{C} 12\right), 82.7\left(C_{\text {spiro }}, \mathrm{C} 17\right), 62.0(\mathrm{CH}, \mathrm{C} 1), 55.8\left(\mathrm{CH}_{3}\right.$, C25), $40.9\left(\mathrm{CH}_{2}, \mathrm{C} 2\right), 30.2\left(\mathrm{CH}_{3}, \mathrm{C} 3\right)$.

\section{3'-methoxy-3-oxo-3H-spiro[isobenzofuran-1,9'-xanthen]-6'-yl 1,2,5-dithiazepane-carboxylate} (SS7-MF, 83)

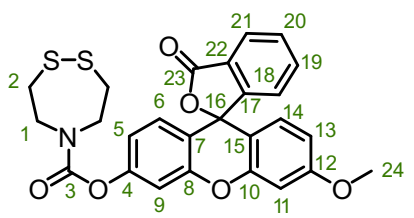

Prepared from crude 49 (corresponding to $400 \mathrm{mg}, 0.642 \mathrm{mmol}$ of compound 47) and an excess of 58 (267 mg, $0.771 \mathrm{mmol}$ ) according to general protocol G to give $83(43 \mathrm{mg}, 0.085 \mathrm{mmol}, 13 \%)$ as a colourless solid after FCC (aluminium oxide, isohexane/EtOAc).

TLC $\mathrm{R}_{f}=0.29$ (isohexane:EtOAc, 2:1). HRMS (ESI $): \mathrm{C}_{26} \mathrm{H}_{22} \mathrm{NO}_{6} \mathrm{~S}_{2}{ }^{+}[\mathrm{M}+\mathrm{H}]^{+}$: calc. 507.0810, found 507.0815.

${ }^{1} \mathrm{H}-N M R\left(400 \mathrm{MHz}, \mathrm{CDCl}_{3}\right): \delta(\mathrm{ppm})=8.02\left(\mathrm{dd},{ }^{3} J_{21-20}=7.3 \mathrm{~Hz},{ }^{4} J_{21-19}=1.1 \mathrm{~Hz}, 1 \mathrm{H}, 21-\mathrm{H}\right), 7.66\left(\mathrm{td},{ }^{3} J_{19-18}=\right.$ $\left.7.4 \mathrm{~Hz},{ }^{4} J_{19-21}=1.4 \mathrm{~Hz}, 1 \mathrm{H}, 19-\mathrm{H}\right), 7.62\left(\mathrm{td},{ }^{3} J_{20-21}=7.4 \mathrm{~Hz},{ }^{4} J_{20-18}=1.2 \mathrm{~Hz}, 1 \mathrm{H}, 20-\mathrm{H}\right), 7.16\left(\mathrm{dt},{ }^{3} J_{18-19}=7.5 \mathrm{~Hz}\right.$, $\left.{ }^{4} J_{18-20}=1.0 \mathrm{~Hz}, 1 \mathrm{H}, 18-\mathrm{H}\right), 7.11\left(\mathrm{~d},{ }^{4} J_{9-5}=2.2 \mathrm{~Hz}, 1 \mathrm{H}, 9-\mathrm{H}\right), 6.82\left(\mathrm{dd},{ }^{3} J_{5-6}=8.7 \mathrm{~Hz},{ }^{4} J_{5-9}=2.2 \mathrm{~Hz}, 1 \mathrm{H}, 5-\mathrm{H}\right), 6.79$ $\left(\mathrm{d},{ }^{3} J_{6-5}=8.4 \mathrm{~Hz}, 1 \mathrm{H}, 6-\mathrm{H}\right), 6.77\left(\mathrm{~d},{ }^{4} J_{11-13}=2.6 \mathrm{~Hz}, 1 \mathrm{H}, 11-\mathrm{H}\right), 6.70\left(\mathrm{~d},{ }^{3} J_{14-13}=8.8 \mathrm{~Hz}, 1 \mathrm{H}, 14-\mathrm{H}\right), 6.62\left(\mathrm{dd},{ }^{3} J_{13-14}=\right.$ $\left.8.8 \mathrm{~Hz},{ }^{4} J_{13-11}=2.5 \mathrm{~Hz}, 1 \mathrm{H}, 13-\mathrm{H}\right), 3.96\left(\mathrm{t},{ }^{3} \mathrm{~J}_{1 \mathrm{~A}-2}=5.8 \mathrm{~Hz}, 2 \mathrm{H}, 1-\mathrm{H}_{\mathrm{A}}\right), 3.88\left(\mathrm{t},{ }^{3} \mathrm{~J}_{1 \mathrm{~B}-2}=5.7 \mathrm{~Hz}, 2 \mathrm{H}, 1-\mathrm{H}_{\mathrm{B}}\right), 3.84(\mathrm{~s}, 3 \mathrm{H}$, 24-H), $3.08\left(\mathrm{t},{ }^{3} \mathrm{~J}_{2-1}=5.9 \mathrm{~Hz}, 4 \mathrm{H}\right)$.

${ }^{13} \mathrm{C}$-NMR $\left(101 \mathrm{MHz}, \mathrm{CDCl}_{3}\right): \delta(\mathrm{ppm})=169.5(\mathrm{C}=\mathrm{O}, \mathrm{C} 23), 161.6\left(C_{\mathrm{Ar}}, \mathrm{C} 12\right), 153.8(C=\mathrm{O}, \mathrm{C} 3), 153.2\left(C_{\mathrm{Ar}}, \mathrm{C} 22\right)$, 152.6 ( $\left.C_{\mathrm{Ar}}, \mathrm{C} 8\right), 152.4\left(C_{\mathrm{Ar}}, \mathrm{C} 15\right), 152.0\left(C_{\mathrm{Ar}}, \mathrm{C} 10\right), 135.2\left(C_{\mathrm{Ar}} \mathrm{H}, \mathrm{C} 19\right), 130.0\left(C_{\mathrm{Ar}} \mathrm{H}, \mathrm{C} 20\right), 129.2\left(C_{\mathrm{Ar}} \mathrm{H}, \mathrm{C} 5 ; \mathrm{C} 14\right)$, $126.6\left(C_{\mathrm{Ar}}, \mathrm{C} 17\right), 125.2\left(C_{\mathrm{Ar}} \mathrm{H}, \mathrm{C} 21\right), 124.2\left(C_{\mathrm{Ar}} \mathrm{H}, \mathrm{C} 18\right), 117.6\left(C_{\mathrm{Ar}} \mathrm{H}, \mathrm{C} 6\right), 116.6\left(C_{\mathrm{Ar}}, \mathrm{C} 7\right), 112.0\left(C_{\mathrm{Ar}} \mathrm{H}, \mathrm{C} 13\right), 111.1$ $\left(C_{\mathrm{Ar}}, \mathrm{C} 4\right), 110.5\left(C_{\mathrm{Ar}} \mathrm{H}, \mathrm{C} 9\right), 101.0\left(C_{\mathrm{Ar}} \mathrm{H}, \mathrm{C} 11\right), 82.7\left(C_{\text {spiro }}, \mathrm{C} 16\right), 55.7\left(\mathrm{CH}_{3}, \mathrm{C} 24\right), 52.1\left(\mathrm{CH}_{2}, \mathrm{C} 1\right), 51.6\left(\mathrm{CH}_{2}, \mathrm{C1}^{\prime}\right)$, $38.3\left(\mathrm{CH}_{2}, \mathrm{C} 2\right), 37.0\left(\mathrm{CH}_{2}, \mathrm{C} 2\right)$.

\section{3'-methoxy-3-oxo-3H-spiro[isobenzofuran-1,9'-xanthen]-6'-yl methyl(2-((2-morpholinoethyl) disulfaneyl) ethyl)carbamate (SSOOM-MF, 84)}

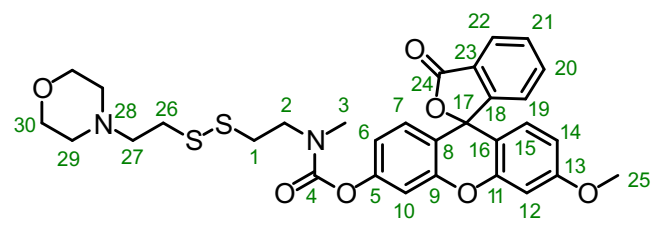

Prepared from $40(25.0 \mathrm{mg}, 0.09 \mathrm{mmol})$ and $\mathbf{5 8}(213 \mathrm{mg}, 0.10 \mathrm{mmol})$ according to general protocol $\mathbf{G}$ to give 84 (133 $\mathrm{mg}, 0.26 \mathrm{mmol}, 65 \%$ ) as solid after FCC (isohexane/EtOAc). The solid was taken into DCM (0.5 mL) and added to $10 \mathrm{~mL}$ of a diluted solution of $\mathrm{HCl}$ in $\mathrm{Et}_{2} \mathrm{O}$, the solid precipitate was separated and dried under reduced pressure to yield $\mathbf{8 4} \cdot \mathrm{HCl}(28.0 \mathrm{mg}, 0.05 \mathrm{mmol}, 56 \%)$ as a colourless solid.

TLC $\mathrm{R}_{f}=0.16$ (EtOAc). HRMS (El'): $\mathrm{C}_{31} \mathrm{H}_{33} \mathrm{~N}_{2} \mathrm{O}_{4} \mathrm{~S}_{2}:$ : [M-e]' calc. 608.1651, found 608.1636.

${ }^{1} \mathrm{H}-\mathrm{NMR}\left(400 \mathrm{MHz}, \mathrm{CDCl}_{3}\right): \delta(\mathrm{ppm})=13.65-12.97(\mathrm{~m}, 1 \mathrm{H}, \mathrm{NH}, 28-\mathrm{H}), 8.02(\mathrm{~d}, J=7.3 \mathrm{~Hz}, 1 \mathrm{H}, 22-\mathrm{H}), 7.68(\mathrm{t}$, $J=7.2 \mathrm{~Hz}, 1 \mathrm{H}, 20-\mathrm{H}), 7.62(\mathrm{t}, J=7.0 \mathrm{~Hz}, 1 \mathrm{H}, 21-\mathrm{H}), 7.17(\mathrm{~d}, J=7.1 \mathrm{~Hz}, 1 \mathrm{H}, 19-\mathrm{H}), 7.14-7.08(\mathrm{~m}, 1 \mathrm{H}, 10-\mathrm{H}), 6.87$ $-6.74(\mathrm{~m}, 3 \mathrm{H}, 6-\mathrm{H} ; 7-\mathrm{H} ; 12-\mathrm{H}), 6.69(\mathrm{dd}, J=8.8,2.3 \mathrm{~Hz}, 1 \mathrm{H}, 15-\mathrm{H}), 6.62(\mathrm{dd}, J=8.8,2.0 \mathrm{~Hz}, 1 \mathrm{H}, 14-\mathrm{H}), 4.30-$ $4.21\left(\mathrm{~m}, 1 \mathrm{H}, 29-\mathrm{H}_{\mathrm{A}}\right), 4.17\left(\mathrm{~d}, J=12.0 \mathrm{~Hz}, 1 \mathrm{H}, 29-\mathrm{H}_{\mathrm{B}}\right), 3.97\left(\mathrm{~d}, J=12.8 \mathrm{~Hz}, 1 \mathrm{H}, 29-\mathrm{H}_{\mathrm{C}}\right), 3.90-3.86\left(\mathrm{~m}, 1 \mathrm{H}, 29-\mathrm{H}_{\mathrm{D}}\right)$, $3.84(\mathrm{~s}, 3 \mathrm{H}, 25-\mathrm{H}), 3.78\left(\mathrm{t}, J=6.5 \mathrm{~Hz}, 1 \mathrm{H}, 1-\mathrm{H}_{\mathrm{A}}\right), 3.69\left(\mathrm{dq}, J=19.9,7.2 \mathrm{~Hz}, 1 \mathrm{H}, 1-\mathrm{H}_{\mathrm{B}}\right), 3.40(\mathrm{~d}, J=10.7 \mathrm{~Hz}, 2 \mathrm{H}$, $30-\mathrm{H}), 3.34-3.21(\mathrm{~m}, 4 \mathrm{H}, 26-\mathrm{H} ; 27-\mathrm{H}), 3.12(\mathrm{~s}, 2 \mathrm{H}, 3-\mathrm{H}$, major rotamer), $3.04(\mathrm{~s}, 1 \mathrm{H}, 3-\mathrm{H}$, minor rotamer), 2.99 (dd, $J=11.9,5.4 \mathrm{~Hz}, 2 \mathrm{H}, 2-\mathrm{H}), 2.86$ (dd, $J=19.5,10.6 \mathrm{~Hz}, 2 \mathrm{H}, 30-\mathrm{H})$.

${ }^{13} \mathrm{C}-\mathrm{NMR}\left(101 \mathrm{MHz}, \mathrm{CDCl}_{3}\right): \delta(\mathrm{ppm})=169.0(C=\mathrm{O}, \mathrm{C} 24), 161.6\left(C_{\mathrm{Ar}}, \mathrm{C} 13\right), 153.8(C=\mathrm{O}, \mathrm{C} 4), 153.0\left(C_{\mathrm{Ar}}, \mathrm{C} 23\right)$, $152.7\left(C_{\mathrm{Ar}}, C 9\right), 152.4\left(C_{\mathrm{Ar}}, C 16\right), 151.9\left(C_{\mathrm{Ar}}, \mathrm{C} 11\right), 135.3\left(C_{\mathrm{Ar}} \mathrm{H}, \mathrm{C} 20\right), 130.0\left(C_{\mathrm{Ar}} \mathrm{H}, \mathrm{C} 21\right), 129.2\left(C_{\mathrm{Ar}} \mathrm{H}, \mathrm{C} 6\right), 129.0$ ( $\left.C_{\mathrm{Ar}} \mathrm{H}, \mathrm{C} 15\right), 126.7\left(C_{\mathrm{Ar}}, \mathrm{C} 18\right), 125.2\left(C_{\mathrm{Ar}} \mathrm{H}, \mathrm{C} 22\right), 124.1\left(C_{\mathrm{Ar}} \mathrm{H}, \mathrm{C} 19\right), 117.7\left(C_{\mathrm{Ar}} \mathrm{H}, \mathrm{C}\right), 116.5\left(C_{\mathrm{Ar}}, \mathrm{C}\right), 112.1$ $\left(C_{\mathrm{Ar}} \mathrm{H}, \mathrm{C} 14\right), 111.0\left(C_{\mathrm{Ar}}, \mathrm{C} 5\right), 110.5\left(C_{\mathrm{Ar}} \mathrm{H}, \mathrm{C} 10\right), 100.9\left(C_{\mathrm{Ar}} \mathrm{H}, \mathrm{C} 12\right), 82.7\left(C_{\text {spiro }}, \mathrm{C} 17\right), 63.7\left(\mathrm{CH}_{2}, \mathrm{C} 29\right), 57.2\left(\mathrm{CH}_{2}\right.$, C27), $55.8\left(\mathrm{CH}_{3}, \mathrm{C} 25\right), 52.3\left(\mathrm{CH}_{2}, \mathrm{C} 30\right), 52.2\left(\mathrm{CH}_{2}, \mathrm{C} 30\right), 48.6\left(\mathrm{CH}_{2}, \mathrm{C} 1\right.$, major rotamer $), 48.0\left(\mathrm{CH}_{2}, \mathrm{C} 1\right.$, major rotamer), $36.3\left(\mathrm{CH}_{2}, \mathrm{C} 2\right), 35.5\left(\mathrm{CH}_{3}, \mathrm{C} 3\right.$, minor rotamer $), 35.3\left(\mathrm{CH}_{3}, \mathrm{C} 3\right.$, major rotamer $), 30.2\left(\mathrm{CH}_{2}, \mathrm{C} 26\right)$. 
bis(3'-methoxy-3-oxo-3H-spiro[isobenzofuran-1,9'-xanthen]-6'-yl) (disulfanediylbis(ethane-2,1diyl))bis(methylcarbamate) $\left(\mathrm{SSOO}_{-} \mathrm{MF}_{2}, 85\right)$

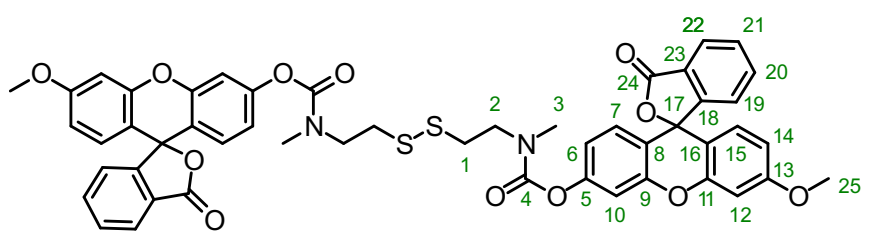

Prepared from $34(96.8 \mathrm{mg}, 0.382 \mathrm{mmol})$ and $58(221.0 \mathrm{mg}, 0.637 \mathrm{mmol})$ according to general protocol $\mathbf{G}$ to give $85(27.3 \mathrm{mg}, 0.026 \mathrm{mmol}, 23 \%)$ as a colourless solid after FCC (isohexane/EtOAc).

TLC $\mathrm{R}_{f}=0.39$ (isohexane:EtOAc, 1:2). $\mathrm{HRMS}\left(\mathrm{ESI}^{+}\right): \mathrm{C}_{50} \mathrm{H}_{41} \mathrm{~N}_{2} \mathrm{O}_{12} \mathrm{~S}_{2}{ }^{+}[\mathrm{M}+\mathrm{H}]^{+}$: calc. 925.20954, found 925.21066.

${ }^{1} \mathrm{H}-\mathrm{NMR}\left(400 \mathrm{MHz}, \mathrm{CDCl}_{3}\right): \delta(\mathrm{ppm})=8.02(\mathrm{~d}, J=7.4 \mathrm{~Hz}, 2 \mathrm{H}, 22-\mathrm{H}), 7.66(\mathrm{t}, J=7.4 \mathrm{~Hz}, 2 \mathrm{H}, 20-\mathrm{H}), 7.61$ (t, $J=7.4 \mathrm{~Hz}, 2 \mathrm{H}, 21-\mathrm{H}), 7.15(\mathrm{dd}, J=7.5,3.2 \mathrm{~Hz}, 2 \mathrm{H}, 19-\mathrm{H}), 7.10(\mathrm{t}, J=1.9 \mathrm{~Hz}, 2 \mathrm{H}, 10-\mathrm{H}), 6.85-6.79(\mathrm{~m}, 2 \mathrm{H}, 6-\mathrm{H})$, $6.79-6.74(\mathrm{~m}, 4 \mathrm{H}, 7-\mathrm{H} ; 12-\mathrm{H}), 6.69(\mathrm{dd}, J=8.8,1.4 \mathrm{~Hz}, 2 \mathrm{H}, 15-\mathrm{H}), 6.62(\mathrm{dd}, J=8.8,2.3 \mathrm{~Hz}, 2 \mathrm{H}, 14-\mathrm{H}), 3.83(\mathrm{~d}$, $J=3.4 \mathrm{~Hz}, 6 \mathrm{H}, 25-\mathrm{H}), 3.75(\mathrm{t}, J=7.0 \mathrm{~Hz}, 2 \mathrm{H}, 1-\mathrm{H}), 3.66\left(\mathrm{t}, J=7.1 \mathrm{~Hz}, 2 \mathrm{H}, 1^{\prime}-\mathrm{H}\right), 3.13(\mathrm{~d}, J=3.6 \mathrm{~Hz}, 3 \mathrm{H}, 3-\mathrm{H}$, major), 3.04 (s, 3H, 3-H, major), 2.94 (q, $J=6.9 \mathrm{~Hz}, 4 \mathrm{H}, 2-\mathrm{H}$ ).

${ }^{13}$ C-NMR $\left(101 \mathrm{MHz}, \mathrm{CDCl}_{3}\right): \delta(\mathrm{ppm})=169.5(C=\mathrm{O}, \mathrm{C} 24), 161.6\left(C_{\mathrm{Ar}}, \mathrm{C} 13\right), 153.9(C=\mathrm{O}, \mathrm{C} 4), 153.2\left(C_{\mathrm{Ar}}, \mathrm{C} 23\right)$, $152.7\left(C_{\mathrm{Ar}}, C 9\right), 152.5\left(C_{\mathrm{Ar}}, \mathrm{C} 16\right), 152.0\left(C_{\mathrm{Ar}}, \mathrm{C} 11\right), 135.2\left(C_{\mathrm{Ar}} \mathrm{H}, \mathrm{C} 20\right), 130.0\left(C_{\mathrm{Ar}} \mathrm{H}, \mathrm{C} 21\right), 129.2\left(C_{\mathrm{Ar}} \mathrm{H}, \mathrm{C} 6\right), 129.0$ $\left(C_{A r} \mathrm{H}, \mathrm{C} 15\right), 126.7\left(C_{\mathrm{Ar}}, \mathrm{C} 18\right), 125.2\left(C_{\mathrm{Ar}} \mathrm{H}, \mathrm{C} 22\right), 124.2\left(C_{\mathrm{Ar}} \mathrm{H}, \mathrm{C} 19\right), 117.7\left(C_{\mathrm{Ar}} \mathrm{H}, \mathrm{C}\right), 116.3\left(C_{\mathrm{Ar}}, \mathrm{C}\right), 112.0$ $\left(C_{A r} \mathrm{H}, \mathrm{C} 14\right), 111.1\left(C_{A r}, C 5\right), 110.4\left(C_{A r} \mathrm{H}, \mathrm{C} 10\right), 101.0\left(C_{A r} \mathrm{H}, \mathrm{C} 12\right), 82.7\left(C_{\text {spiro }}, \mathrm{C} 17\right), 55.7\left(\mathrm{CH}_{3}, \mathrm{C} 25\right), 49.2\left(\mathrm{CH}_{2}\right.$, C1), $\left.48.6\left(\mathrm{CH}_{2}, \mathrm{C}\right)^{\prime}\right), 36.4\left(\mathrm{CH}_{2}, \mathrm{C} 2\right), 35.8\left(\mathrm{CH}_{2}, \mathrm{C} 2^{\prime}\right), 35.6\left(\mathrm{CH}_{3}, \mathrm{C} 3\right)$. 


\section{IG-Series}

cis-2',7'-difluoro-3'-isopropoxy-3-oxo-3H-spiro[isobenzofuran-1,9'-xanthen]-6'-yl-hexahydro$[1,2]$ dithiino[4,5-b]pyridine-1(2H)-carboxylate (SS66C-IG, 86)
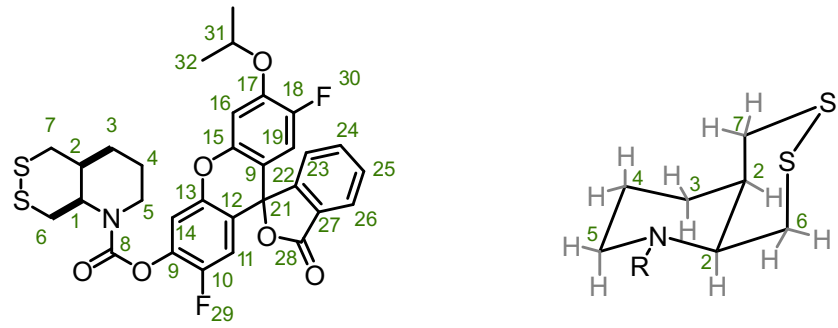

Prepared from crude 12 (corresponding to $41 \mathrm{mg}, 0.14 \mathrm{mmol}$ of 18 ) and 62 (48 $\mathrm{mg}, 0.12 \mathrm{mmol}$ ) according to general protocol $\mathbf{G}$ to give 86 (26 mg, $0.043 \mathrm{mmol}, 36 \%)$ as a colourless solid FCC (isohexane/EtOAc).

TLC $\mathrm{R}_{f}=0.67$ (isohexane:EtOAc, 2:1). HRMS $\left(\mathrm{ESI}^{+}\right) \mathrm{C}_{31} \mathrm{H}_{38} \mathrm{~F}_{2} \mathrm{NO}_{6} \mathrm{~S}_{2}{ }^{+}[\mathrm{M}+\mathrm{H}]^{+}$: calc. 612.13206, found 612.13221.

Individual rotamers were observed by NMR spectroscopy at $298 \mathrm{~K}$ (ratio ca. 1:1). The following data refers to all signals observed at $298 \mathrm{~K}$.

${ }^{1} \mathrm{H}-N M R\left(400 \mathrm{MHz}, \mathrm{CDCl}_{3}\right): \delta(\mathrm{ppm})=8.04(\mathrm{~d}, J=7.4 \mathrm{~Hz}, 1 \mathrm{H}, 26-\mathrm{H}), 7.72(\mathrm{tt}, J=7.5,1.5 \mathrm{~Hz}, 2 \mathrm{H}, 24-\mathrm{H}), 7.66(\mathrm{t}$, $J=7.4 \mathrm{~Hz}, 1 \mathrm{H}, 25-\mathrm{H}), 7.19(\mathrm{~d}, J=7.5 \mathrm{~Hz}, 1 \mathrm{H}, 23-\mathrm{H}), 7.15(\mathrm{dd}, J=6.5,4.1 \mathrm{~Hz}, 1 \mathrm{H}, 14-\mathrm{H}), 6.83(\mathrm{~d}, J=7.1 \mathrm{~Hz}, 1 \mathrm{H}$, $16-\mathrm{H}), 6.53(\mathrm{dd}, J=10.1,3.3 \mathrm{~Hz}, 1 \mathrm{H}, 11-\mathrm{H}), 6.44(\mathrm{~d}, J=11.2 \mathrm{~Hz}, 1 \mathrm{H}, 19-\mathrm{H}), 4.62$ (hept, $J=6.5 \mathrm{~Hz}, 1 \mathrm{H}, 31-\mathrm{H})$, $4.58-4.47(\mathrm{~m}, 1 \mathrm{H}, 1-\mathrm{H}), 4.12\left(\mathrm{dd}, J=27.8,13.8 \mathrm{~Hz}, 1 \mathrm{H}, 5-\mathrm{H}_{\mathrm{A}}\right), 3.61-3.41\left(\mathrm{~m}, 2 \mathrm{H}, 6-\mathrm{H}_{\mathrm{A}} ; 7-\mathrm{H}_{\mathrm{A}}\right), 3.15-2.89(\mathrm{~m}$, $\left.1 \mathrm{H}, 5-\mathrm{H}_{\mathrm{B}}\right), 2.88-2.75\left(\mathrm{~m}, 1 \mathrm{H}, 6-\mathrm{H}_{\mathrm{B}}\right), 2.62-2.45\left(\mathrm{~m}, 1 \mathrm{H}, 7-\mathrm{H}_{\mathrm{B}}\right), 2.41-2.22\left(\mathrm{~m}, 2 \mathrm{H}, 2-\mathrm{H} ; 3-\mathrm{H}_{\mathrm{A}}\right), 1.86(\mathrm{~d}, \mathrm{~J}=11.5 \mathrm{~Hz}$, $\left.1 \mathrm{H}, 4-\mathrm{H}_{\mathrm{A}}\right), 1.76-1.64\left(\mathrm{~m}, 1 \mathrm{H}, 4-\mathrm{H}_{\mathrm{B}}\right), 1.52\left(\mathrm{dd}, J=9.3,5.4 \mathrm{~Hz}, 1 \mathrm{H}, 3-\mathrm{H}_{\mathrm{B}}\right), 1.41(\mathrm{~d}, J=6.0 \mathrm{~Hz}, 6 \mathrm{H}, 32-\mathrm{H})$.

${ }^{13} \mathrm{C}$-NMR $(101 \mathrm{MHz}, \mathrm{CDCl}): \delta(\mathrm{ppm})=168.8(C=\mathrm{O}, \mathrm{C} 28), 152.2\left(C_{\mathrm{Ar}}, \mathrm{C} 27\right), 152.1(C=\mathrm{O}, \mathrm{C} 8), 150.8\left(C_{\mathrm{Ar}}, \mathrm{C} 17\right)$, $148.7\left(C_{\mathrm{Ar}}, \mathrm{C} 20\right), 148.4\left(\mathrm{~d}, J_{C-F}=12.5 \mathrm{~Hz}, C_{\mathrm{Ar}}, \mathrm{C} 18\right), 147.7\left(\mathrm{~d}, J_{C-F}=27.0 \mathrm{~Hz}, C_{\mathrm{Ar}}, \mathrm{C} 10\right), 140.7\left(C_{\mathrm{Ar}}, \mathrm{C}\right), 135.6$ $\left(C_{\mathrm{Ar}} \mathrm{H}, \mathrm{C} 25\right), 130.5\left(C_{\mathrm{Ar}} \mathrm{H}, \mathrm{C} 24\right), 126.4\left(C_{\mathrm{Ar}}, \mathrm{C} 22\right), 125.6\left(C_{\mathrm{Ar}} \mathrm{H}, \mathrm{C} 26\right), 124.6\left(C_{\mathrm{Ar}} \mathrm{H}, \mathrm{C} 23\right), 116.2\left(C_{\mathrm{Ar}}, \mathrm{C} 12\right), 114.7$ $\left(\mathrm{d}, J_{C-F}=21.6 \mathrm{~Hz}, C_{A r} \mathrm{H}, \mathrm{C} 19\right), 114.1\left(\mathrm{~d}, J_{C-F}=21.6 \mathrm{~Hz}, C_{\mathrm{Ar}} \mathrm{H}, \mathrm{C} 11\right), 112.9\left(\mathrm{~d}, J_{C-F}=18.0 \mathrm{~Hz}, C_{\mathrm{Ar}} \mathrm{H}, \mathrm{C} 14\right), 109.3$ $\left(C_{\mathrm{Ar}}, \mathrm{C} 17\right), 104.0\left(\mathrm{C}_{\mathrm{Ar}} \mathrm{H}, \mathrm{C} 19\right), 82.6\left(C_{\text {spiro }}, \mathrm{C} 21\right), 72.4(\mathrm{CH}, \mathrm{C} 31), 54.6(\mathrm{CH}, \mathrm{C} 1$; major $), 53.9(\mathrm{CH}, \mathrm{C} 1$; minor $), 42.3$ ( $\mathrm{CH}_{2}, \mathrm{C} 6$, minor), $42.1\left(\mathrm{CH}_{2}, \mathrm{C} 6\right.$, major $), 41.0\left(\mathrm{CH}_{2}, \mathrm{C} 5\right.$, minor $), 40.5\left(\mathrm{CH}_{2}, \mathrm{C} 5\right.$, major $), 36.4(\mathrm{CH}, \mathrm{C} 2$; minor $), 36.1$ ( $\mathrm{CH}$, C2; minor), $30.0\left(\mathrm{CH}_{2}, \mathrm{C} 7\right.$, minor $), 29.4\left(\mathrm{CH}_{2}, \mathrm{C} 7\right.$, minor $), 25.4\left(\mathrm{CH}_{2}, \mathrm{C} 4\right.$, minor $), 25.0\left(\mathrm{CH}_{2}, \mathrm{C} 4\right.$, major $), 23.5$ $\left(\mathrm{CH}_{2}, \mathrm{C} 3\right), 22.0\left(\mathrm{CH}_{3}, \mathrm{C} 32\right), 21.9\left(\mathrm{CH}_{3}, \mathrm{C} 32\right)$.

cis-2',7'-difluoro-3'-isopropoxy-3-oxo-3H-spiro[isobenzofuran-1,9'-xanthen]-6'-yl hexahydrofuro [3,4-b]pyridine-1(2H)-carboxylate (O56-IG, 87)

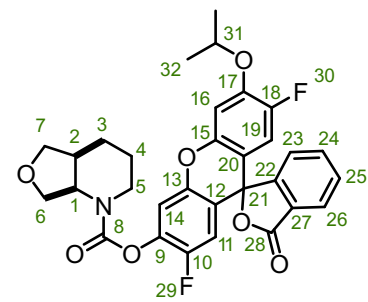

Prepared from $19(25 \mathrm{mg}, 0.15 \mathrm{mmol})$ and $62(48 \mathrm{mg}, 0.12 \mathrm{mmol})$ according to general protocol G to give 87 (35 mg, $0.062 \mathrm{mmol}, 52 \%$ ) as a colourless solid after FCC (isohexane/EtOAc).

TLC $\mathrm{R}_{f}=0.26$ (isohexane:EtOAc, 2:1). HRMS (ESI $\left.{ }^{+}\right): \mathrm{C}_{31} \mathrm{H}_{28} \mathrm{~F}_{2} \mathrm{NO}_{7}^{+}[\mathrm{M}+\mathrm{H}]^{+}$: calc. 564.1829, found 564.1825.

${ }^{1} \mathrm{H}-N M R\left(400 \mathrm{MHz}, \mathrm{CDCl}_{3}\right): \delta(\mathrm{ppm})=8.04(\mathrm{~d}, J=7.4 \mathrm{~Hz}, 1 \mathrm{H}, 26-\mathrm{H}), 7.72(\mathrm{t}, J=7.4 \mathrm{~Hz}, 1 \mathrm{H}, 24-\mathrm{H}), 7.67$ (t, $J=7.4 \mathrm{~Hz}, 1 \mathrm{H}, 25-\mathrm{H}), 7.22-7.12(\mathrm{~m}, 2 \mathrm{H}, 23-\mathrm{H} ; 14-\mathrm{H}), 6.83(\mathrm{~d}, J=7.1 \mathrm{~Hz}, 1 \mathrm{H}, 16-\mathrm{H}), 6.53(\mathrm{~d}, J=10.1 \mathrm{~Hz}, 1 \mathrm{H}$, $11-\mathrm{H}), 6.44(\mathrm{dd}, J=11.3,1.1 \mathrm{~Hz}, 1 \mathrm{H}, 19-\mathrm{H}), 4.95-4.75(\mathrm{~m}, 1 \mathrm{H}, 1-\mathrm{H}), 4.62$ (hept, $J=6.0 \mathrm{~Hz}, 1 \mathrm{H}, 31-\mathrm{H}), 4.19-$ $4.08\left(\mathrm{~m}, 1 \mathrm{H}, 5-\mathrm{H}_{\mathrm{A}}\right), 4.10-4.01\left(\mathrm{~m}, 1 \mathrm{H}, 6-\mathrm{H}_{\mathrm{A}}\right), 3.92\left(\mathrm{~s}, 1 \mathrm{H}, 7-\mathrm{H}_{\mathrm{A}}\right), 3.73\left(\mathrm{~d}, J=6.4 \mathrm{~Hz}, 2 \mathrm{H}, 6-\mathrm{H}_{\mathrm{B}} ; 7-\mathrm{H}_{\mathrm{B}}\right), 3.16-2.85$ $\left(\mathrm{m}, 1 \mathrm{H}, 5-\mathrm{H}_{\mathrm{B}}\right), 2.38-2.20(\mathrm{~m}, 1 \mathrm{H}, 2-\mathrm{H}), 1.94-1.76\left(\mathrm{~m}, 2 \mathrm{H}, 3-\mathrm{H}_{\mathrm{A}} ; 4-\mathrm{H}_{\mathrm{A}}\right), 1.61-1.49\left(\mathrm{~m}, 2 \mathrm{H}, 3-\mathrm{H}_{\mathrm{B}} ; 4-\mathrm{H}_{\mathrm{B}}\right), 1.41(\mathrm{~d}$, $J=6.0 \mathrm{~Hz}, 6 \mathrm{H}, 32-\mathrm{H})$.

${ }^{13} \mathrm{C}-N M R\left(101 \mathrm{MHz}, \mathrm{CDCl}_{3}\right): \delta(\mathrm{ppm})=167.5(\mathrm{C}=\mathrm{O}, \mathrm{C} 28), 152.1(\mathrm{C}=\mathrm{O}, \mathrm{C} 8), 151.2\left(C_{\mathrm{Ar}}, \mathrm{C} 17\right), 148.7\left(C_{\mathrm{Ar}}, \mathrm{C} 20\right)$, $148.4\left(\mathrm{~d}, J_{C-F}=12.5 \mathrm{~Hz}, C_{\mathrm{Ar}}, \mathrm{C} 18\right), 147.7\left(\mathrm{~d}, J_{C-F}=32.2 \mathrm{~Hz}, C_{\mathrm{Ar}}, \mathrm{C} 10\right), 140.7\left(C_{\mathrm{Ar}}, \mathrm{C} 9\right), 135.6\left(C_{\mathrm{Ar}} \mathrm{H}, \mathrm{C} 25\right), 130.5$ $\left(C_{\mathrm{Ar}} \mathrm{H}, \mathrm{C} 24\right), 126.4\left(C_{\mathrm{Ar}}, \mathrm{C} 22\right), 125.6\left(C_{\mathrm{Ar}} \mathrm{H}, \mathrm{C} 26\right), 124.1\left(C_{\mathrm{Ar}} \mathrm{H}, \mathrm{C} 23\right), 115.9\left(C_{\mathrm{Ar}}, \mathrm{C} 12\right), 114.7\left(\mathrm{~d}, J_{C-F}=21.5 \mathrm{~Hz}\right.$, $\left.C_{A r} H, C 19\right), 114.1\left(d, J_{C-F}=21.5 \mathrm{~Hz}, C_{A r} H, C 11\right), 112.9\left(C_{A r} H, C 14\right), 109.3\left(C_{A r}, C 17\right), 104.0\left(C_{A r} H, C 19\right), 82.1$ ( $\left.\mathrm{C}_{\text {spiro, }} \mathrm{C} 21\right), 73.4\left(\mathrm{CH}_{2}, \mathrm{C} 7\right), 72.4(\mathrm{CH}, \mathrm{C} 31), 66.3\left(\mathrm{CH}_{2}, \mathrm{C} 6\right), 54.5(\mathrm{CH}, \mathrm{C} 1), 41.4\left(\mathrm{CH}_{2}, \mathrm{C} 5\right), 35.9(\mathrm{CH}, \mathrm{C} 2), 26.5$ $\left(\mathrm{CH}_{2}, \mathrm{C} 3\right), 23.9\left(\mathrm{CH}_{2}, \mathrm{C} 4\right), 22.0\left(\mathrm{CH}_{3}, \mathrm{C} 32\right)$. 


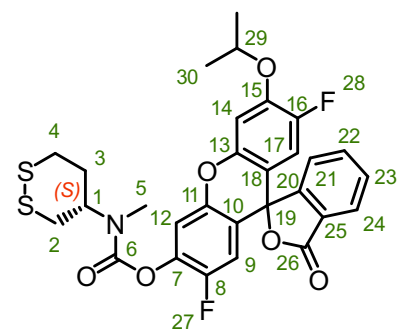

Prepared from $25(28.0 \mathrm{mg}, 0.150 \mathrm{mmol})$ and $62(48.0 \mathrm{mg}, 0.12 \mathrm{mmol})$ according to general protocol $\mathbf{G}$ to give $88(38.0 \mathrm{mg}, 0.065 \mathrm{mmol}, 54 \%)$ as a colourless solid after FCC (isohexane/EtOAc).

TLC $\mathrm{R}_{f}=0.57$ (isohexane:EtOAc, 2:1). $\mathrm{HRMS}\left(\mathrm{ESI}^{+}\right): \mathrm{C}_{29} \mathrm{H}_{26} \mathrm{~F}_{2} \mathrm{NO}_{6} \mathrm{~S}_{2}{ }^{+}[\mathrm{M}+\mathrm{H}]^{+}$: calc. 586.11641, found 586.11609. Individual rotamers were observed by NMR spectroscopy at $298 \mathrm{~K}$ (ratio ca. 1.5:1). The following data refers to all signals observed at $298 \mathrm{~K}$.

${ }^{1} \mathrm{H}-\mathrm{NMR}\left(400 \mathrm{MHz}, \mathrm{CDCl}_{3}\right): \delta(\mathrm{ppm})=8.04(\mathrm{~d}, J=7.4 \mathrm{~Hz}, 1 \mathrm{H}, 24-\mathrm{H}), 7.72(\mathrm{td}, J=7.4,1.2 \mathrm{~Hz}, 1 \mathrm{H}, 22-\mathrm{H}), 7.66(\mathrm{t}$, $J=7.4 \mathrm{~Hz}, 1 \mathrm{H}, 23-\mathrm{H}), 7.15(\mathrm{t}, J=6.0 \mathrm{~Hz}, 1 \mathrm{H}, 21-\mathrm{H}), 7.15(\mathrm{dd}, J=6.5,4.1 \mathrm{~Hz}, 1 \mathrm{H}, 12-\mathrm{H}), 6.83(\mathrm{~d}, J=7.1 \mathrm{~Hz}, 1 \mathrm{H}$, $14-\mathrm{H}), 6.53$ (dd, $J=10.1,2.8 \mathrm{~Hz}, 1 \mathrm{H}, 9-\mathrm{H}), 6.44(\mathrm{~d}, J=11.2 \mathrm{~Hz}, 1 \mathrm{H}, 17-\mathrm{H}), 4.62$ (hept, $J=6.1 \mathrm{~Hz}, 29-\mathrm{H}), 4.30$ (ddd, $J=17.7,10.5,4.7 \mathrm{~Hz}, 1 \mathrm{H}, 1-\mathrm{H}), 3.22$ (ddd, $J=14.1,8.7,2.5 \mathrm{~Hz}, 1 \mathrm{H}, 2-\mathrm{H}_{\mathrm{A}}$ ), 3.14 (dd, $J=12.1,2.5 \mathrm{~Hz}, 1 \mathrm{H}$, $\left.3-\mathrm{H}_{\mathrm{A}}\right), 3.07-2.97\left(\mathrm{~m}, 3 \mathrm{H}, 2-\mathrm{H}_{\mathrm{B}} ; 5-\mathrm{H}\right), 2.95(\mathrm{~s}, 1 \mathrm{H}, 5$ ' $-\mathrm{H}), 2.89-2.74\left(\mathrm{~m}, 1 \mathrm{H}, 3-\mathrm{H}_{\mathrm{B}}\right), 2.24(\mathrm{dd}, J=29.3,13.2 \mathrm{~Hz}$, $\left.1 \mathrm{H}, 4-\mathrm{H}_{\mathrm{A}}\right), 2.14-2.06\left(\mathrm{~m}, 1 \mathrm{H}, 4-\mathrm{H}_{\mathrm{B}}\right), 1.41(\mathrm{~d}, J=6.1 \mathrm{~Hz}, 6 \mathrm{H}, 30-\mathrm{H})$.

${ }^{13} \mathrm{C}-\mathrm{NMR}\left(101 \mathrm{MHz}, \mathrm{CDCl}_{3}\right): \delta(\mathrm{ppm})=168.8(C=\mathrm{O}, \mathrm{C} 26), 152.8(C=\mathrm{O}, \mathrm{C} 6), 152.6\left(C=\mathrm{O}, 6^{\prime}-\mathrm{C}\right), 152.2\left(C_{\mathrm{Ar}}, \mathrm{C} 25\right)$, $149.6\left(C_{\mathrm{Ar}}, \mathrm{C} 15\right), 148.7\left(C_{\mathrm{Ar}}, \mathrm{C} 18\right), 148.4\left(\mathrm{~d}, J_{\mathrm{C}-\mathrm{F}}=12.5 \mathrm{~Hz}, C_{\mathrm{Ar}}, \mathrm{C} 16\right), 147.7\left(\mathrm{~d}, J_{\mathrm{C}-\mathrm{F}}=28.2 \mathrm{~Hz}, C_{\mathrm{Ar}}, \mathrm{C} 8\right), 140.7$ $\left(C_{\mathrm{Ar}}, \mathrm{C} 7\right), 135.6\left(C_{\mathrm{Ar}} \mathrm{H}, \mathrm{C} 23\right), 130.5\left(C_{\mathrm{Ar}} \mathrm{H}, \mathrm{C} 22\right), 126.4\left(C_{\mathrm{Ar}}, \mathrm{C} 20\right), 125.6\left(C_{\mathrm{Ar}} \mathrm{H}, \mathrm{C} 24\right), 124.1\left(C_{\mathrm{Ar}} \mathrm{H}, \mathrm{C} 21\right), 116.5$ $\left(C_{A r}, C 10\right), 114.7\left(\mathrm{~d}, J_{C-F}=21.4 \mathrm{~Hz}, C_{A_{\mathrm{r}}} \mathrm{H}, \mathrm{C} 17\right), 114.1\left(\mathrm{~d}, J_{C-F}=21.5 \mathrm{~Hz}, C_{\mathrm{Ar}} \mathrm{H}, \mathrm{C} 9\right), 112.9\left(\mathrm{~d}, J_{C-F}=18.0 \mathrm{~Hz}, C_{\mathrm{Ar}} \mathrm{H}\right.$, C12), 109.3 ( $\left.C_{\mathrm{Ar}}, \mathrm{C} 15\right), 104.0\left(\mathrm{C}_{\mathrm{Ar}} \mathrm{H}, \mathrm{C} 17\right), 82.1$ ( $\left.\mathrm{C}_{\text {spiro }}, \mathrm{C} 19\right), 72.4(\mathrm{CH}, \mathrm{C} 29), 56.4(\mathrm{CH}, \mathrm{C}$; ; minor), $56.0(\mathrm{CH}, \mathrm{C} 1$; major), 36.2 ( $\mathrm{CH}_{2}, \mathrm{C} 2$, major), $36.1\left(\mathrm{CH}_{2}, \mathrm{C} 2\right.$, minor), $35.6\left(\mathrm{CH}_{2}, \mathrm{C} 3\right), 33.0\left(\mathrm{CH}_{2}, \mathrm{C} 4\right.$, major), $32.4\left(\mathrm{CH}_{2}, \mathrm{C} 4\right.$, minor $)$, $30.3\left(\mathrm{CH}_{3}, \mathrm{C} 5\right), 21.9\left(\mathrm{CH}_{3}, \mathrm{C} 30\right)$. 
1,2-dithian-4-yl (SS60-MR, 90)<smiles>COc1ccc2c(c1)Oc1cc(N)ccc1C21OC(=O)c2ccccc21</smiles>

64<smiles>COc1ccc2c(c1)OC(=O)c1ccccc1C21NC(=O)Oc2ccccc21</smiles><smiles>COc1ccc2c(c1)OC(=O)c1ccccc1C21c2ccccc2Oc2cc(NC(=O)OC3CCSC3)ccc21</smiles>

90

Step 1: To a solution of 64 (50.0 mg, $0.145 \mathrm{mmol}, 1.0$ eq.) and pyridine $(23.5 \mu \mathrm{L}, 0.291 \mathrm{mmol}, 2.0$ eq.) in anhydrous $\mathrm{DCM}(3 \mathrm{~mL})$ at $0{ }^{\circ} \mathrm{C}$ was added 4-nitrophenyl chloroformate $(35.0 \mathrm{mg}, 0.174 \mathrm{mmol}, 1.2 \mathrm{eq}$.) and the mixture was allowed to warm to r.t. and was further stirred for $15 \mathrm{~h}$. Then, the reaction mixture was directly subjected to FCC (0 to $5 \%$ EtOAc in DCM), which afforded partially purified $89(62 \mathrm{mg}$ ) as a yellow solids. This material still contaminated a significant amount 4-nitrophenol and was used without further purification in the next step.

Step 2: The material obtained in step $1(20 \mathrm{mg})$ containing 89 and 30 (7.5 mg, $0.055 \mathrm{mmol})$ were dissolved in anhydrous acetone $(0.4 \mathrm{~mL})$. DBU $(7 \mu \mathrm{L}, 0.05 \mathrm{mmol})$ was added and the mixture was stirred for $5 \mathrm{~h}$, before the volatiles were removed under reduced pressure. The residue was dissolved in DCM $(5 \mathrm{~mL})$, washed with half-sat. aq. $\mathrm{K}_{2} \mathrm{CO}_{3}$ until the aqueous layer stayed colourless. Then, the organic layers were dried over $\mathrm{Na}_{2} \mathrm{SO}_{4}$, filtered and again evaporated under reduced pressure. The residue was purified by FCC (EtOAc:isohexane:DCM = 1:4:4) to afford 90 (17.5 mg, $0.035 \mathrm{mmol}, 74 \%$ over two steps) as a colourless solid.

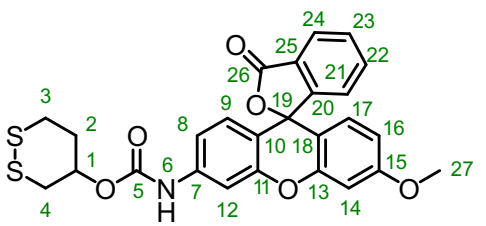

TLC $\mathrm{R}_{f}=0.27$ (EtOAc:hexane:DCM, 1:4:4). HRMS (ESI): $\mathrm{C}_{22} \mathrm{H}_{26} \mathrm{NO}_{6} \mathrm{~S}_{2}{ }^{+}[\mathrm{M}+\mathrm{H}]^{+}$calc. 508.08831, found 508.08880.

${ }^{1} \mathrm{H}-N M R(400 \mathrm{MHz}$, DMSO-d 6 ): $\delta(\mathrm{ppm})=10.13(\mathrm{~s}, 1 \mathrm{H}, 6-\mathrm{H}), 8.02(\mathrm{~d}, J=7.7 \mathrm{~Hz}, 1 \mathrm{H}, 24-\mathrm{H}), 7.80$ (td, $J=7.5$, $1.3 \mathrm{~Hz}, 1 \mathrm{H}, 22-\mathrm{H}), 7.73(\mathrm{td}, J=7.4,1.0 \mathrm{~Hz}, 1 \mathrm{H}, 23-\mathrm{H}), 7.58(\mathrm{~s}, 1 \mathrm{H}, 13-\mathrm{H}), 7.28(\mathrm{~d}, J=7.6 \mathrm{~Hz}, 1 \mathrm{H}, 21-\mathrm{H}), 7.20(\mathrm{dt}$, $J=8.8,1.5 \mathrm{~Hz}, 1 \mathrm{H}, 17-\mathrm{H}), 7.00(\mathrm{~d}, J=2.5 \mathrm{~Hz}, 1 \mathrm{H}, 12-\mathrm{H}), 6.76-6.70(\mathrm{~m}, 2 \mathrm{H}, 8-\mathrm{H} ; 16-\mathrm{H}), 6.67(\mathrm{~d}, J=8.8 \mathrm{~Hz}, 1 \mathrm{H}$, $9-\mathrm{H}), 4.87(\mathrm{td}, J=9.8,8.9,4.1 \mathrm{~Hz}, 1 \mathrm{H}, 1-\mathrm{H}), 3.82(\mathrm{~s}, 3 \mathrm{H}, 27-\mathrm{H}), 3.25-3.14\left(\mathrm{~m}, 2 \mathrm{H}, 2-\mathrm{H}_{\mathrm{A}} ; 4-\mathrm{H}_{\mathrm{A}}\right), 3.08(\mathrm{t}, J=12.1 \mathrm{~Hz}$, $\left.1 \mathrm{H}, 2-\mathrm{H}_{\mathrm{A}}\right), 2.86\left(\mathrm{dd}, J=13.0,9.6 \mathrm{~Hz}, 1 \mathrm{H}, 4-\mathrm{H}_{\mathrm{B}}\right), 2.40-2.27\left(\mathrm{~m}, 1 \mathrm{H}, 3-\mathrm{H}_{\mathrm{A}}\right), 1.84\left(\mathrm{q}, J=11.3,10.6 \mathrm{~Hz}, 1 \mathrm{H}, 3-\mathrm{H}_{\mathrm{B}}\right)$.

${ }^{13} \mathrm{C}$-NMR (101 MHz, DMSO-d $)$ : $\delta(\mathrm{ppm})=169.1(C=0), 161.6(C=0), 153.0\left(C_{\mathrm{Ar}}\right), 152.7\left(C_{\mathrm{Ar}}\right), 152.2\left(C_{\mathrm{Ar}}\right), 151.4$ $\left(C_{\mathrm{Ar}}\right), 141.8\left(C_{\mathrm{Ar}}\right), 136.2\left(C_{\mathrm{Ar}} \mathrm{H}, \mathrm{C}-22\right), 130.7\left(C_{\mathrm{Ar}} \mathrm{H}, \mathrm{C}-23\right), 129.4\left(C_{\mathrm{Ar}} \mathrm{H}, \mathrm{C}-9\right), 129.1\left(C_{\mathrm{Ar}} \mathrm{H}, \mathrm{C}-8\right), 126.3\left(C_{\mathrm{Ar}}\right), 125.2$ $\left(C_{\mathrm{Ar}} \mathrm{H}, \mathrm{C}-25\right), 124.4\left(C_{\mathrm{Ar}} \mathrm{H}, \mathrm{C}-21\right), 115.0\left(C_{\mathrm{Ar}} \mathrm{H}, \mathrm{C}-17\right), 113.0\left(C_{\mathrm{Ar}}\right), 112.6\left(C_{\mathrm{Ar}} \mathrm{H}, \mathrm{C}-16\right), 111.2\left(C_{\mathrm{Ar}}\right), 105.6\left(C_{\mathrm{Ar}} \mathrm{H}\right.$, C-13), $101.3\left(\mathrm{C}_{\mathrm{Ar}} \mathrm{H}, \mathrm{C}-12\right), 82.6\left(\mathrm{C}_{\text {spiro, }} \mathrm{C}-19\right), 70.9(\mathrm{CH}, \mathrm{C}-1), 56.2\left(\mathrm{CH}_{3}, \mathrm{C}-27\right), 36.8\left(2 \times \mathrm{CH}_{2}, \mathrm{C}-2 ; \mathrm{C}-4\right), 33.8\left(\mathrm{CH}_{2}\right.$, C-3). 


\section{NMR Spectra}

dimethyl cis-N-Boc-piperidine-2,3-dicarboxylate (2)

${ }^{1} \mathrm{H}-\mathrm{NMR}(373 \mathrm{~K})$

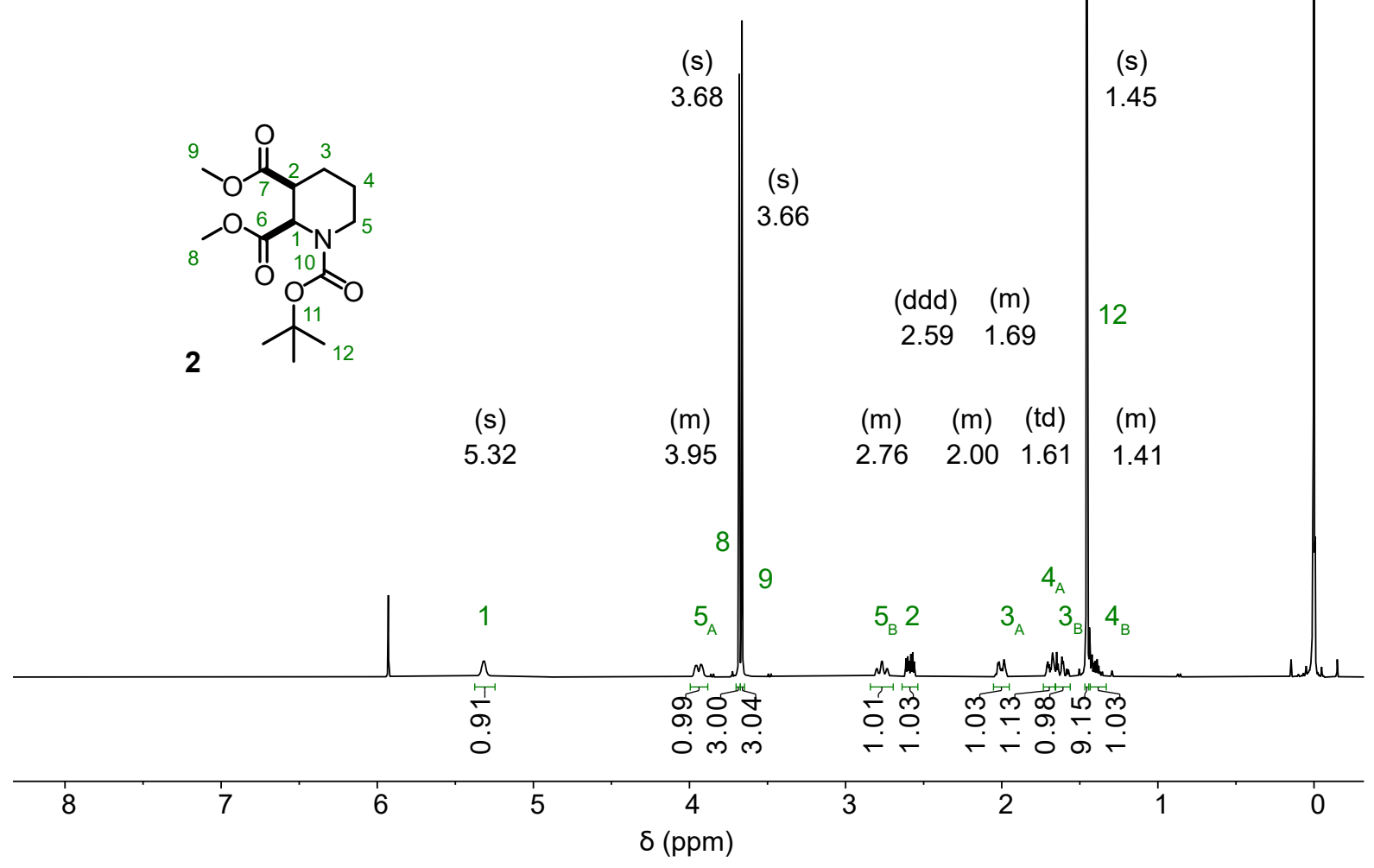

${ }^{13} \mathrm{C}-\mathrm{NMR}(373 \mathrm{~K})$
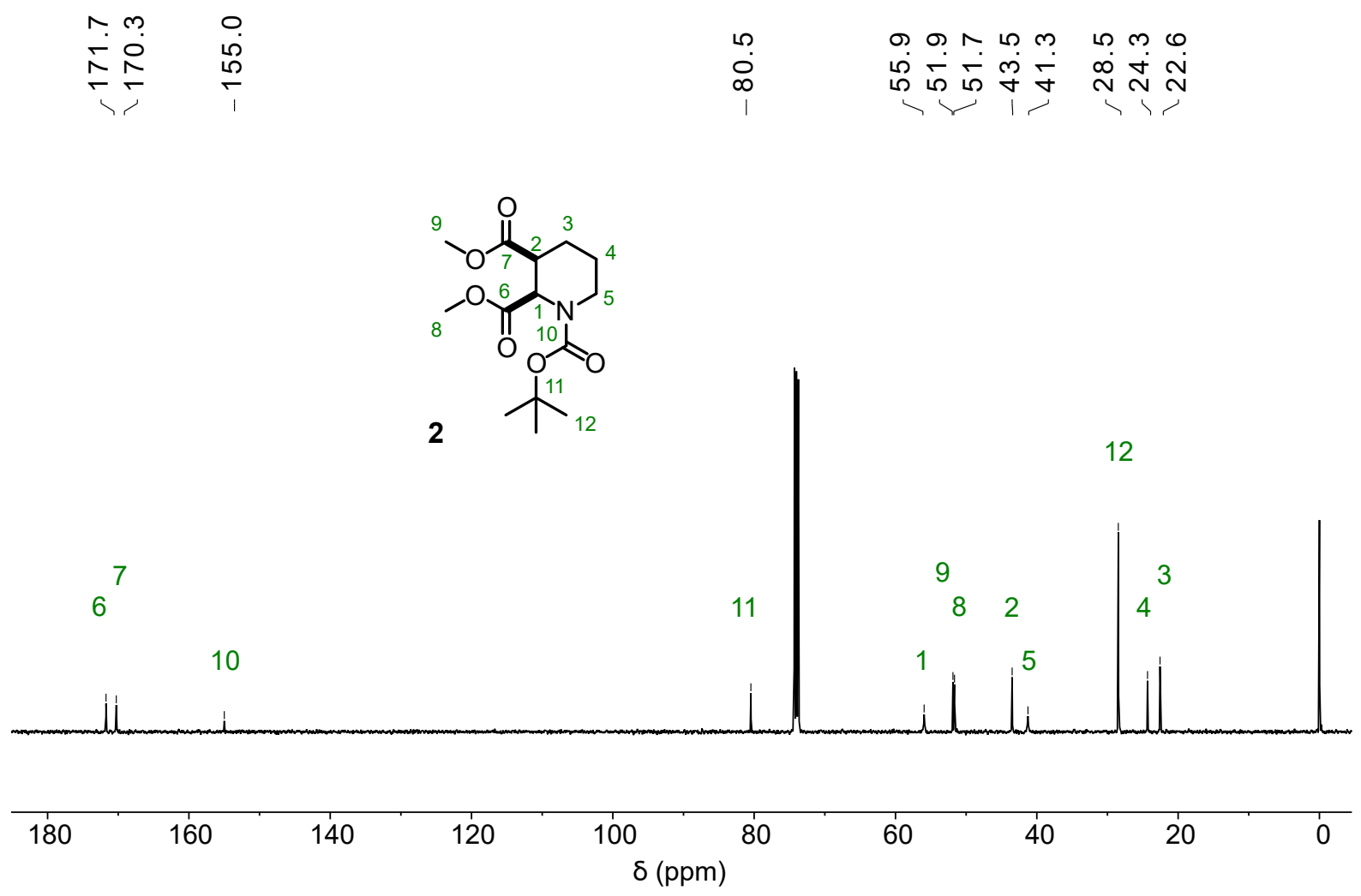
cis/trans-epimerisation: dimethyl N-Boc-piperidine-2,3-dicarboxylate (14)
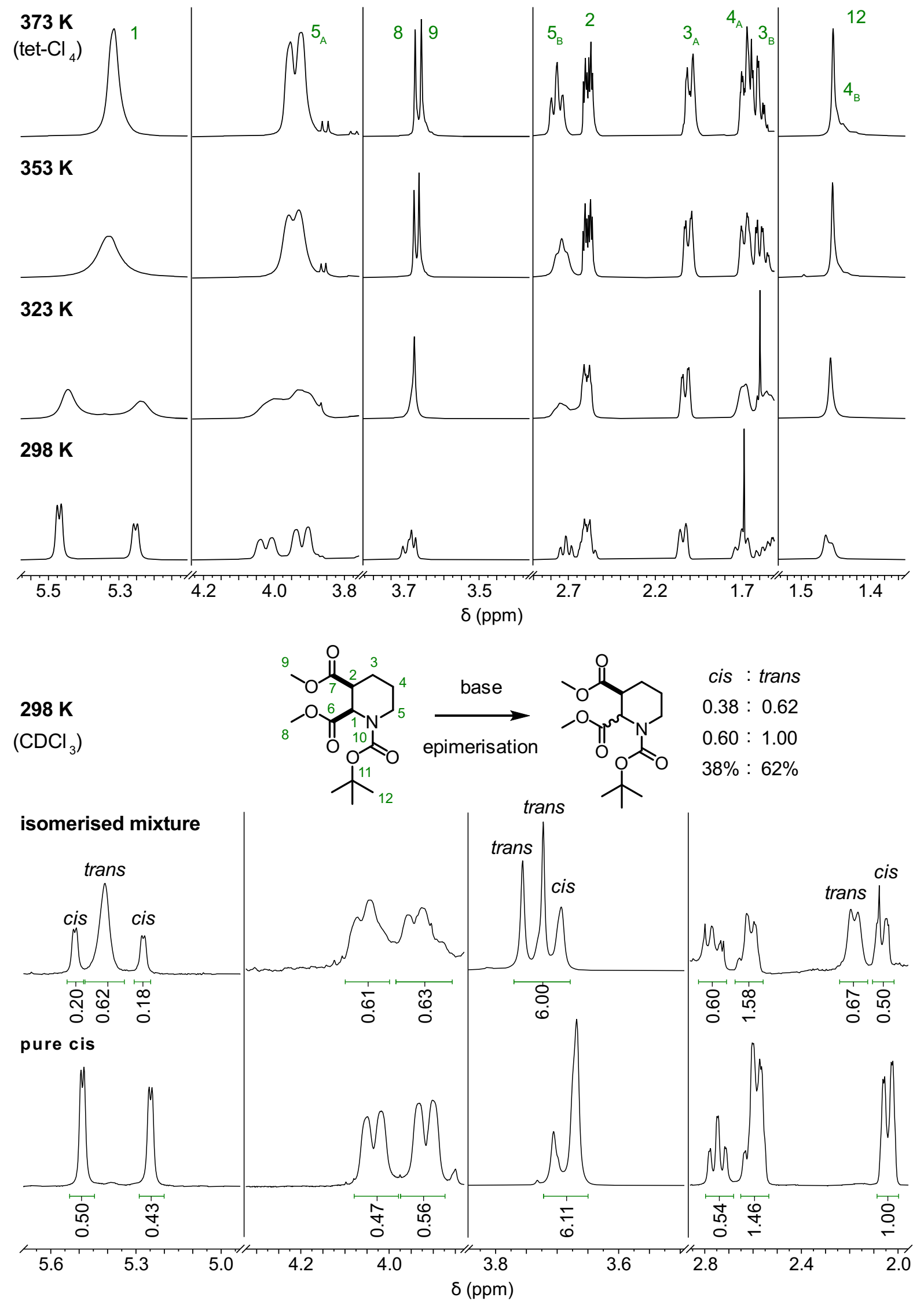
cis-N-Boc 2,3-bis(hydroxymethyl)piperidine (3)

${ }^{1} \mathrm{H}-\mathrm{NMR}$

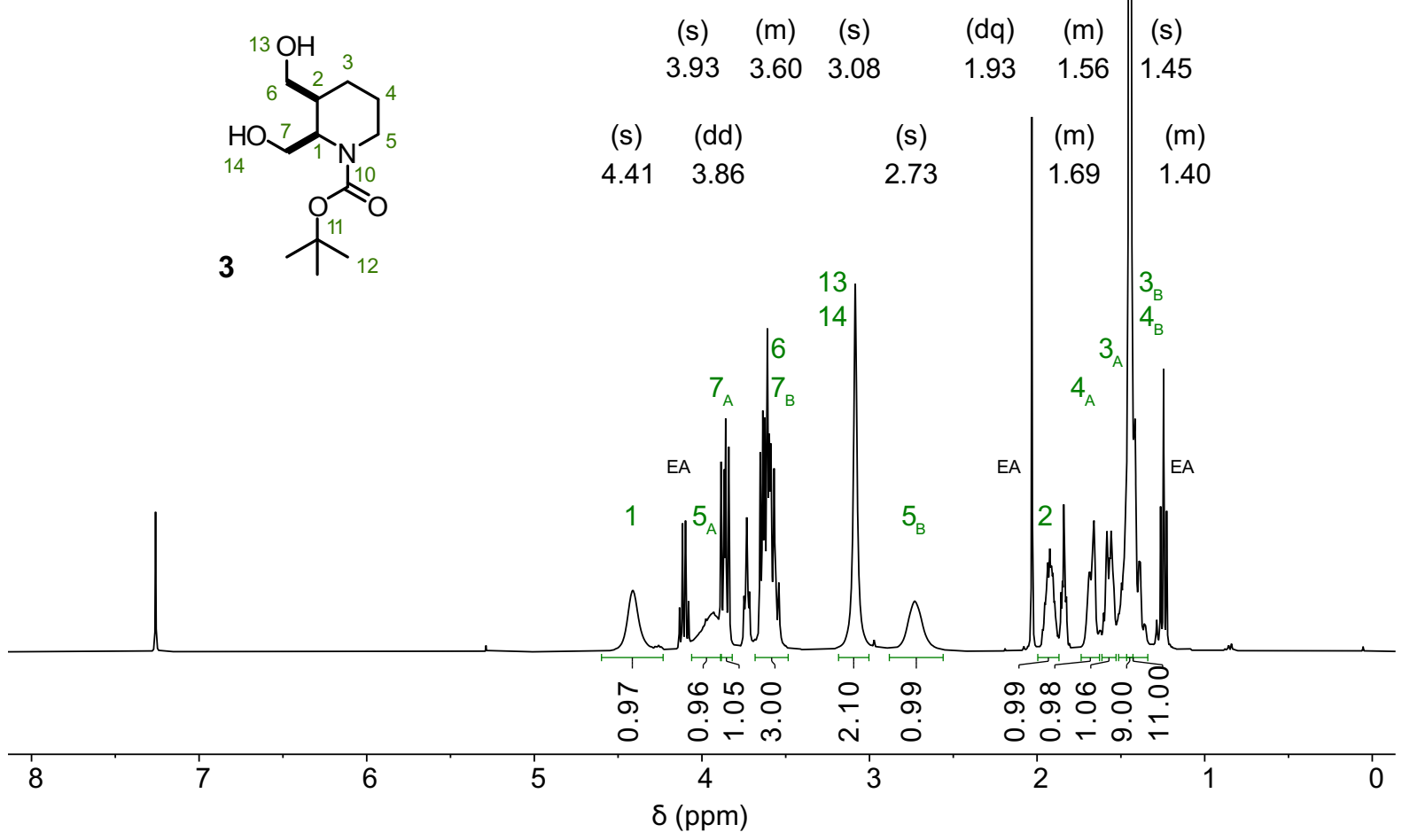

${ }^{13} \mathrm{C}-\mathrm{NMR}$

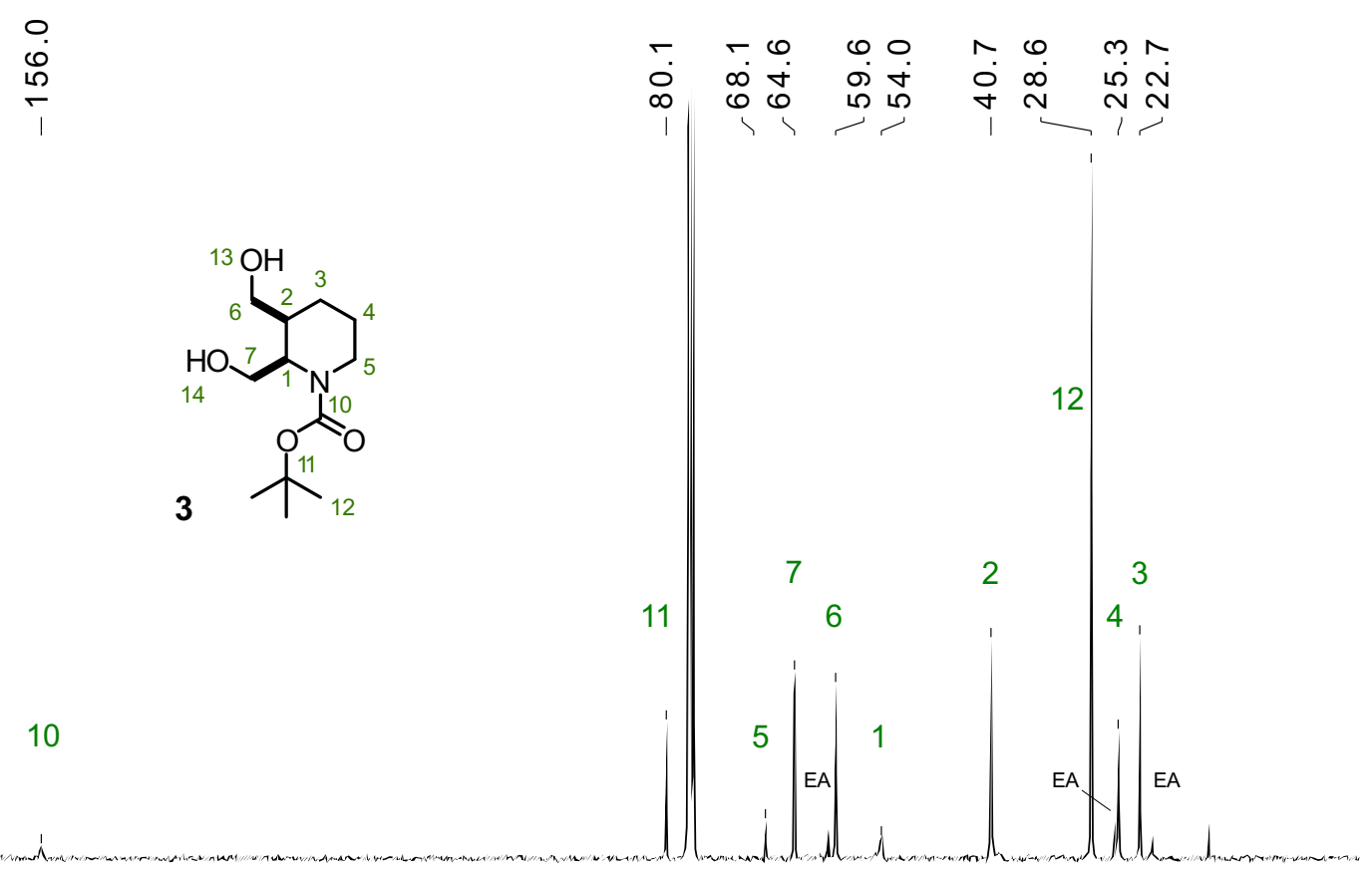

\begin{tabular}{cccccccccc}
\hline 180 & 160 & 140 & 120 & 100 & 80 & 60 & 40 & 20 & 0 \\
\hline$(\mathrm{ppm})$ &
\end{tabular}


cis-N-Boc hexahydrofuro[3,4-b]pyridine (5)

${ }^{1} \mathrm{H}-\mathrm{NMR}$

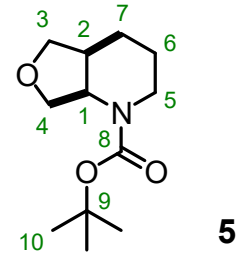

$\begin{array}{llll}(\mathrm{dd}) & (\mathrm{t}) \quad(\mathrm{m}) \quad(\mathrm{m})\end{array}$

\begin{tabular}{lll|l}
3.64 & 2.74 & 1.74 & 1.41
\end{tabular}

(s) $\quad(\mathrm{m}) \quad(\mathrm{t}) \quad(\mathrm{m}) \quad(\mathrm{m})$

$4.65 \quad 3.873 .56 \quad 2.111 .68$

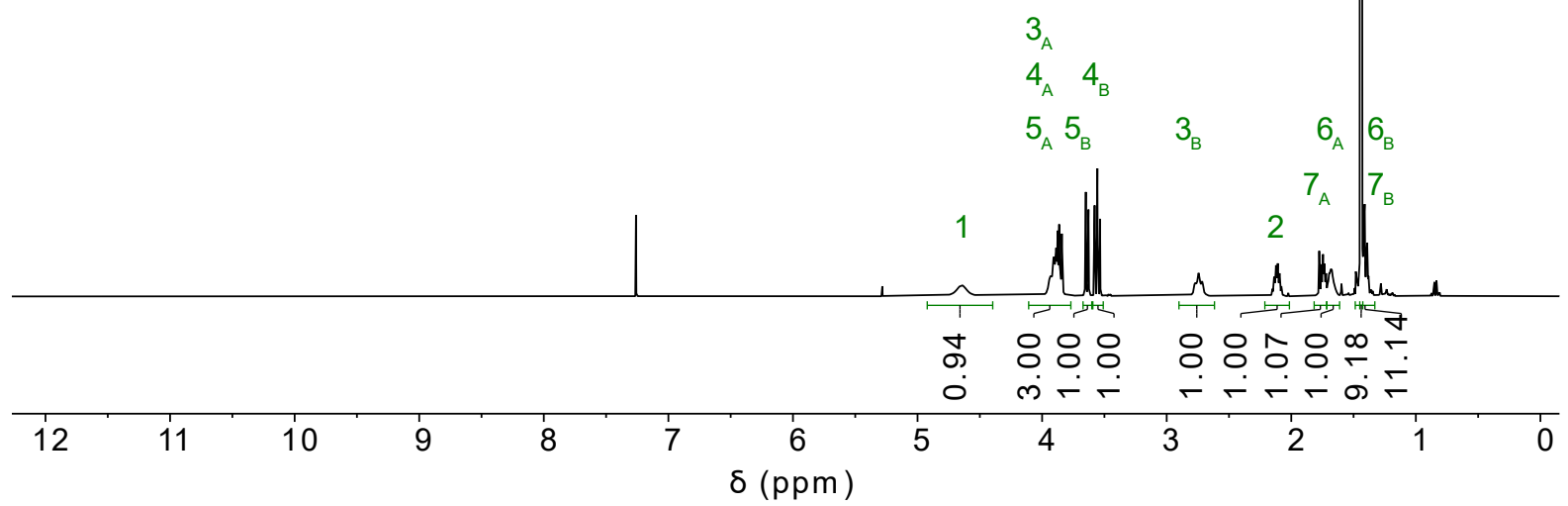

${ }^{13} \mathrm{C}-\mathrm{NMR}$

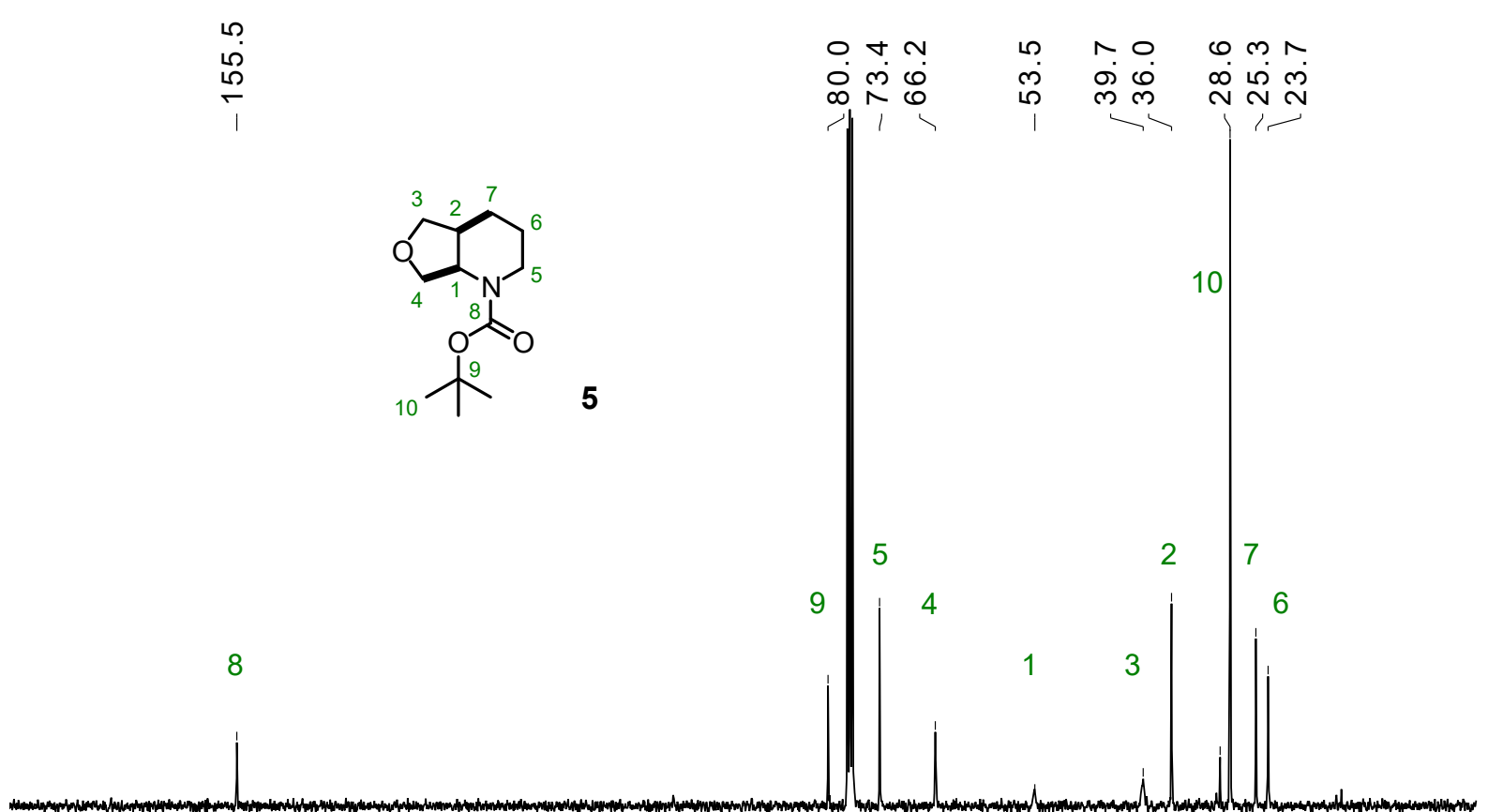

$180 \quad 160$

140

120

100

$\delta(\mathrm{ppm})$

20 
trans-N-Boc 2,3-bis((acetylthio)methyl)piperidine (7)

${ }^{1} \mathrm{H}-\mathrm{NMR}$

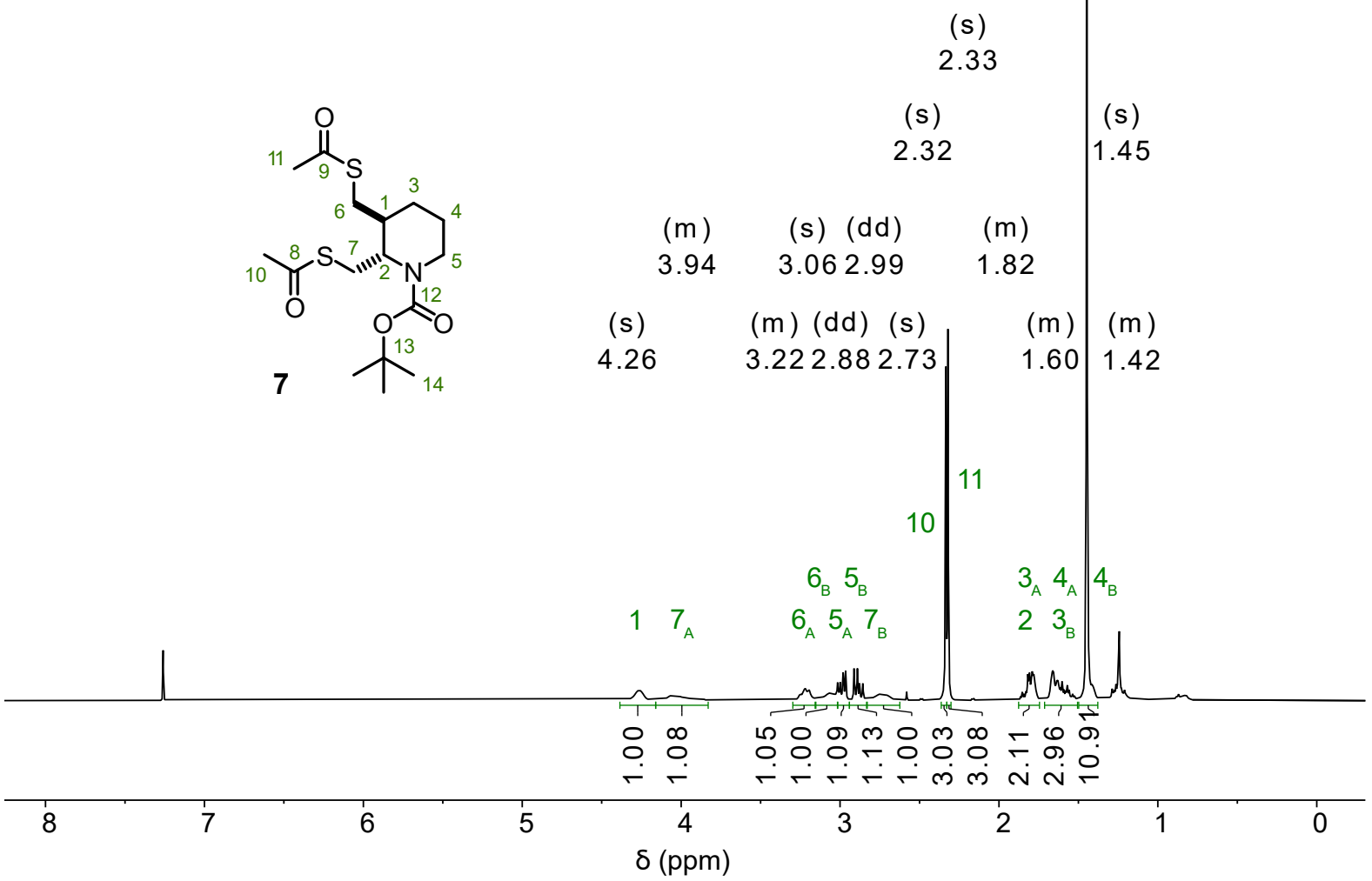

${ }^{13} \mathrm{C}-\mathrm{NMR}$

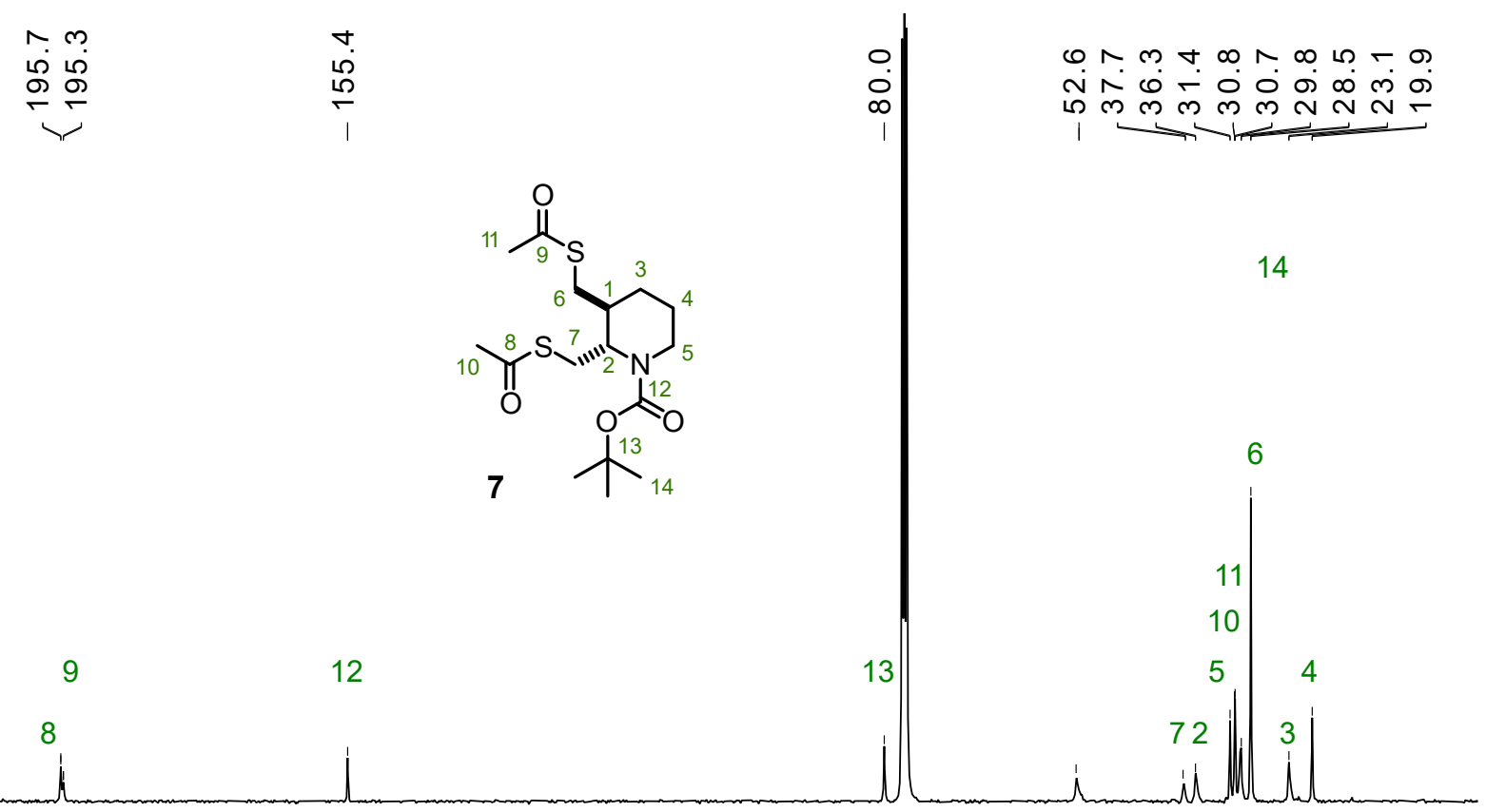

\begin{tabular}{lllllclllll}
\hline 200 & 180 & 160 & 140 & 120 & $\begin{array}{c}100 \\
\delta(\mathrm{ppm})\end{array}$ & 80 & 60 & 40 & 20 & 0
\end{tabular}


trans-hexahydro-[1,2]dithiino[4,5-b]pyridine hydrochloride (8)

${ }^{1} \mathrm{H}-\mathrm{NMR}$

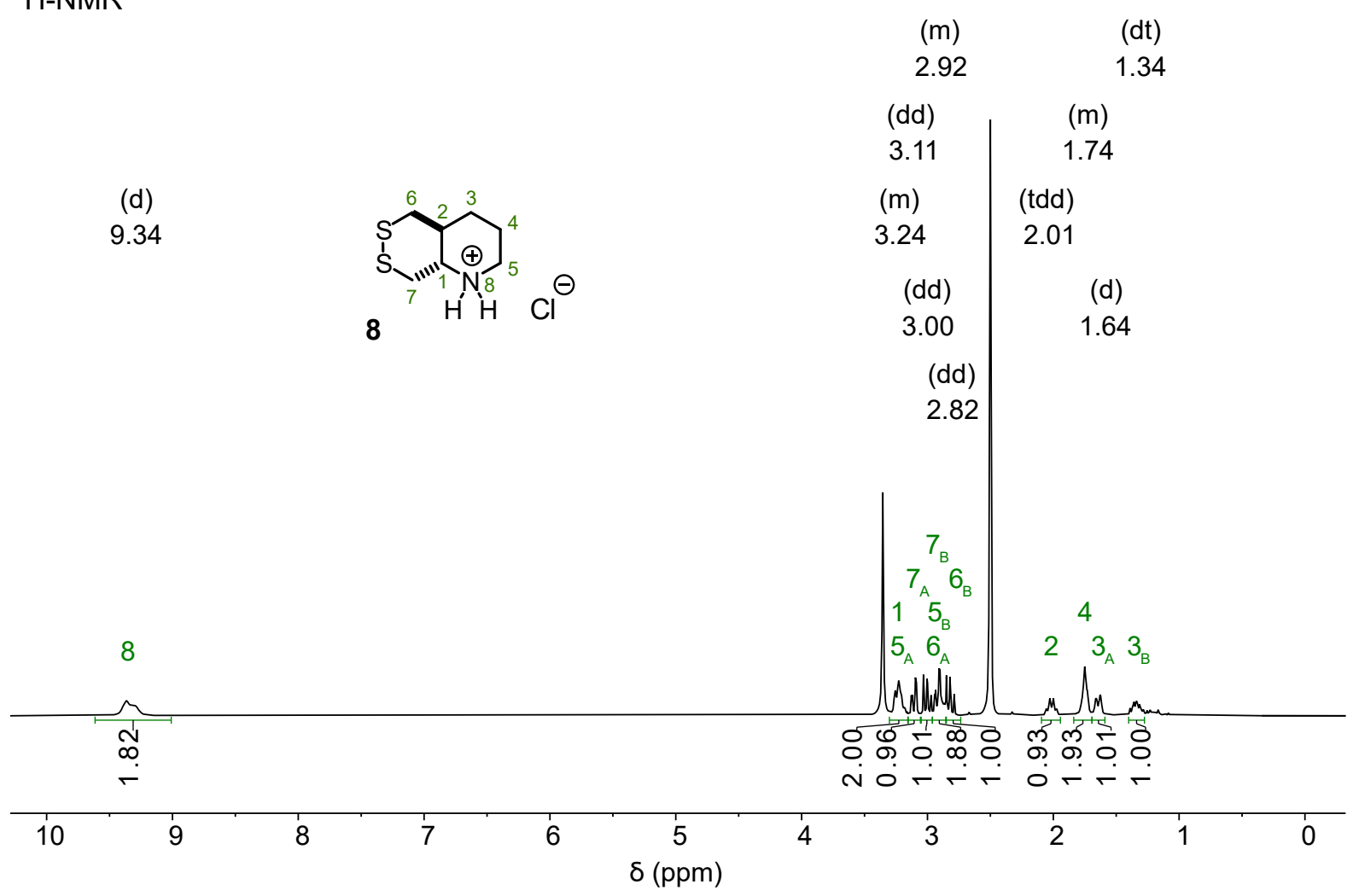

${ }^{13} \mathrm{C}-\mathrm{NMR}$

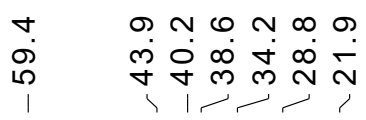

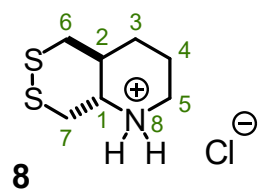

$\begin{array}{lll}7 & 3 & 4\end{array}$

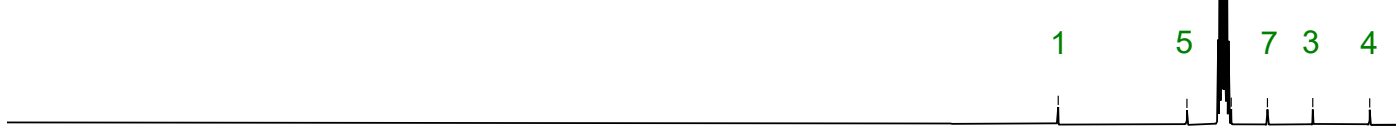

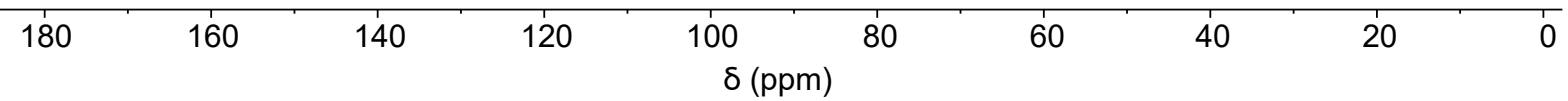


cis-N-(4-methoxybenzyl)-2,3-bis(hydroxymethyl)piperidine (10)

${ }^{1} \mathrm{H}-\mathrm{NMR}$

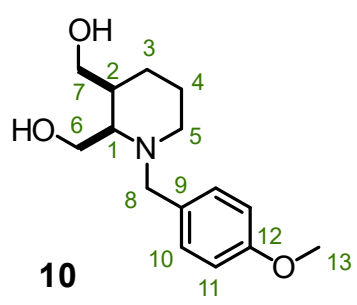

(d)

6.86

(d)

7.24

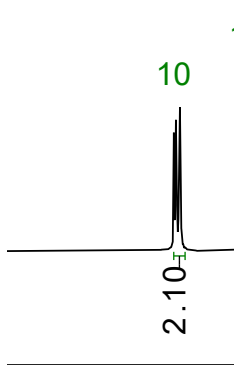

8

7 (s)

3.79 (t)

3.813 .71
13

(d) $\quad(m) \quad(m)$

$3.86 \quad 3.60 \quad 2.95$

$2.49 \quad 1.66$

(ddd) (dd) (m)

$\begin{array}{lll}2.74 & 2.17 & 1.45\end{array}$

$\delta(\mathrm{ppm})$

${ }^{13} \mathrm{C}-\mathrm{NMR}$

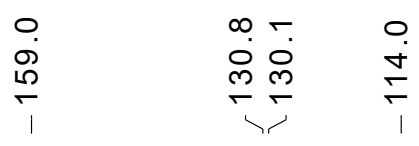

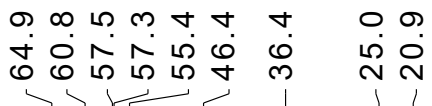

10<smiles>COc1ccc(CN2CCC[C@@H](CO)[C@H]2CO)cc1</smiles>

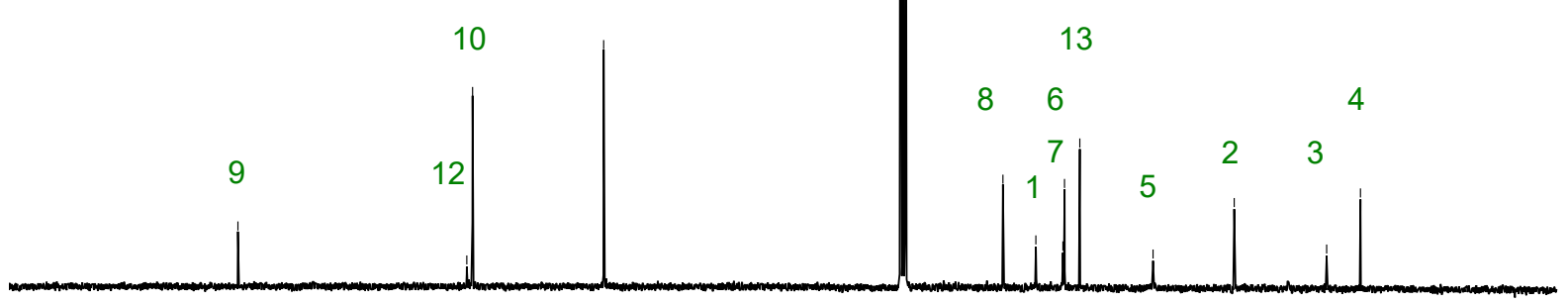

180

160

140

120

100

$\delta(\mathrm{ppm})$

80

60

40

0

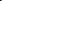


cis-N-((1-(4-methoxybenzyl)piperidine-2,3-diyl)bis(methylene)) diethanethioate (11)

${ }^{1} \mathrm{H}-\mathrm{NMR}$
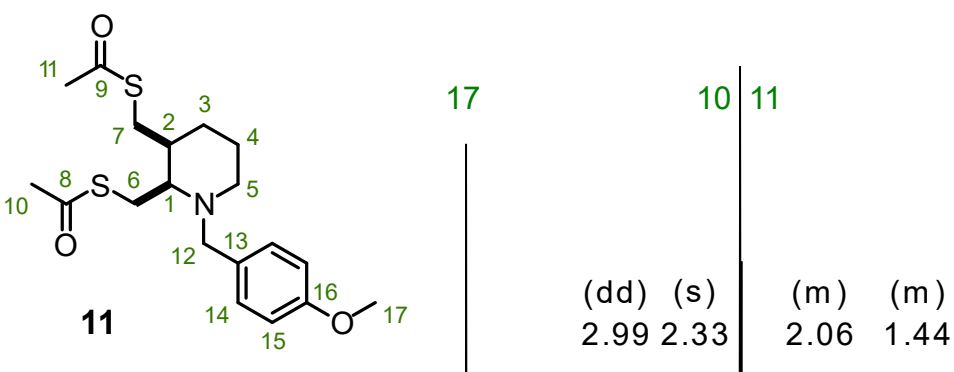

(d)

6.84

$\begin{array}{lllll}(\mathrm{s}) & (\mathrm{dt}) & (\mathrm{td}) & (\mathrm{s}) \quad(\mathrm{m})\end{array}$

(dd) (s) $\quad(\mathrm{m})-(\mathrm{m})$

\begin{tabular}{ll|ll}
3.67 & 2.912 .60 & 2.33 & 1.58
\end{tabular}

(d)

(s)

7.24

3.80

(dd) $(m)(d)$

3.252 .832 .44

(m) (m)

$1.68 \quad 1.35$

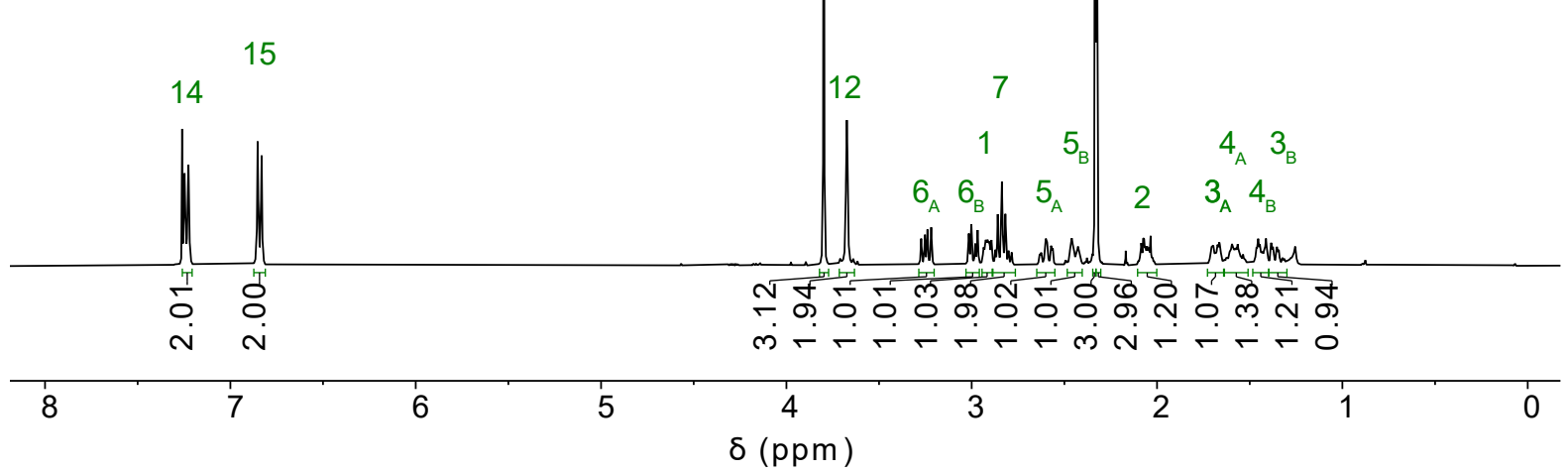

${ }^{13} \mathrm{C}-\mathrm{NMR}$

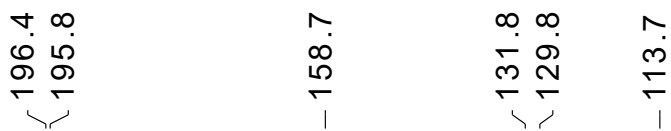

$00 \forall m 000$, 0 lo m

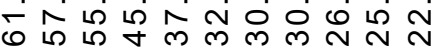<smiles>COc1ccc(CN2CCC[C@H](CSC(C)=O)[C@@H]2CSC(C)=O)cc1</smiles>

$14 \quad 15$

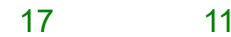

12

21064

89

13

16

573

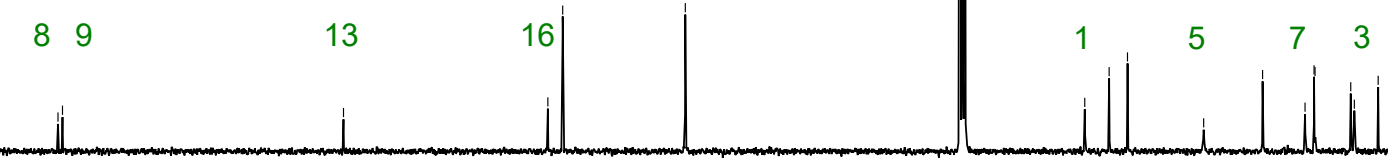

200

180

160

140

120

100

60

$\delta(\mathrm{ppm})$ 
cis-N-(4-methoxybenzyl)-octahydro-[1,2]dithiino[4,5-b]pyridine (12)

${ }^{1} \mathrm{H}-\mathrm{NMR}$

(d)

6.87

12<smiles>COc1ccc(CN2CCCC3CSCC[C@H]32)cc1</smiles>

(d)

7.22

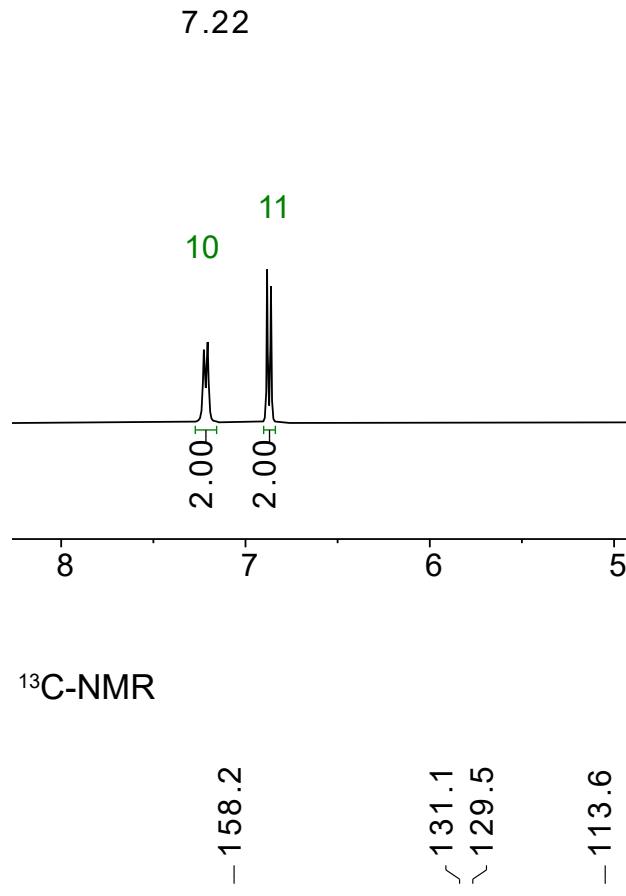

(s)

3.73

(m) (dd)

$3.34 \quad 2.70$

(d)

(d)

(m) (d) (m)

$\begin{array}{llll}3.66 & 2.94 & 2.392 .091 .53\end{array}$

(d)

3.52

(d) (d)

3.252 .84

(m) (m) (d)

2.171 .591 .34

4
$\delta(p p m)$

0 क०

móñorir

$\begin{array}{llll}3 & 2 & 1 & 0\end{array}$
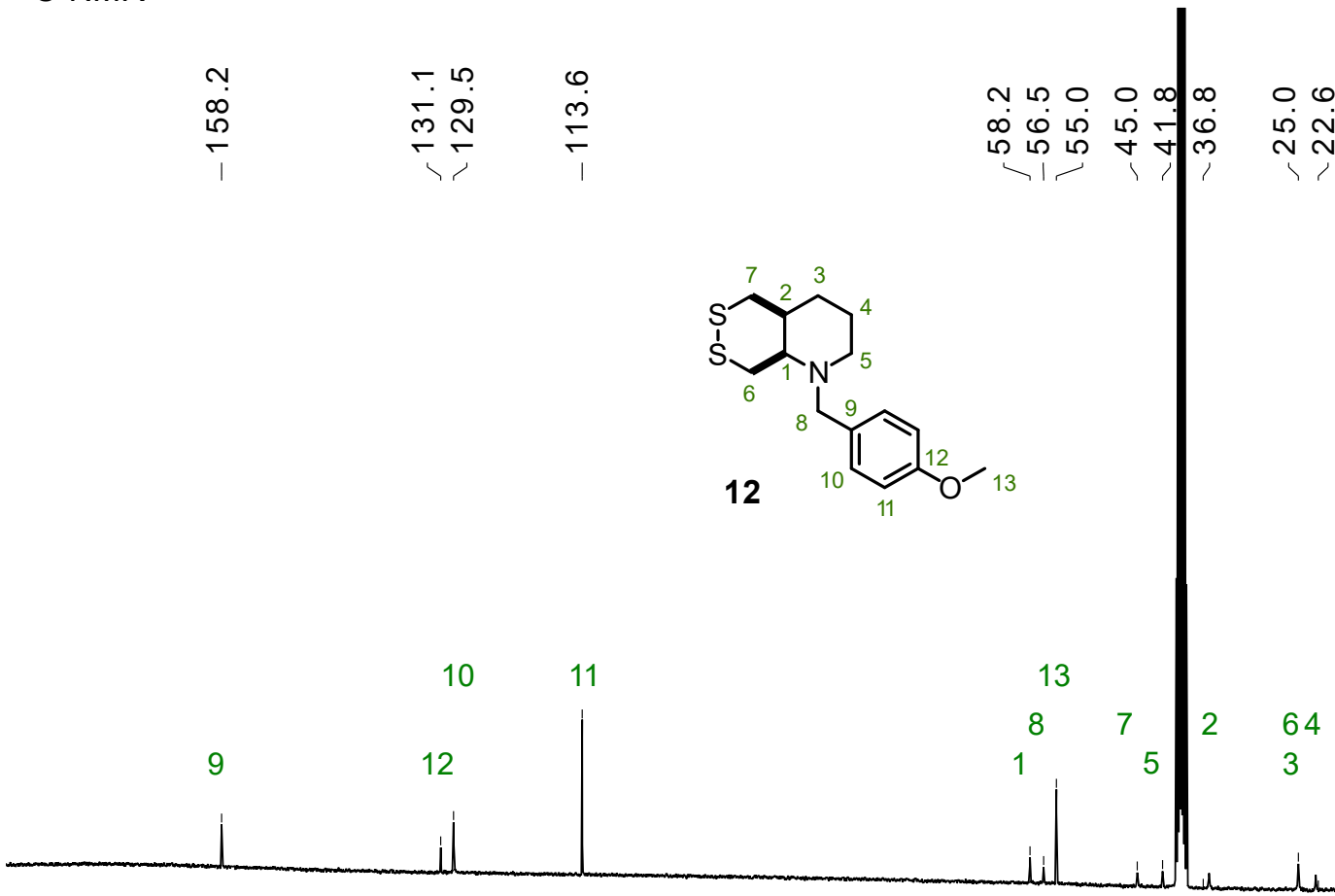

$180 \quad 160$

140

120

100

$\delta(p p m)$ 
trans-N-Boc hexahydro-[1,2]dithiino[4,5-b]pyridine (15)

${ }^{1} \mathrm{H}-\mathrm{NMR}$
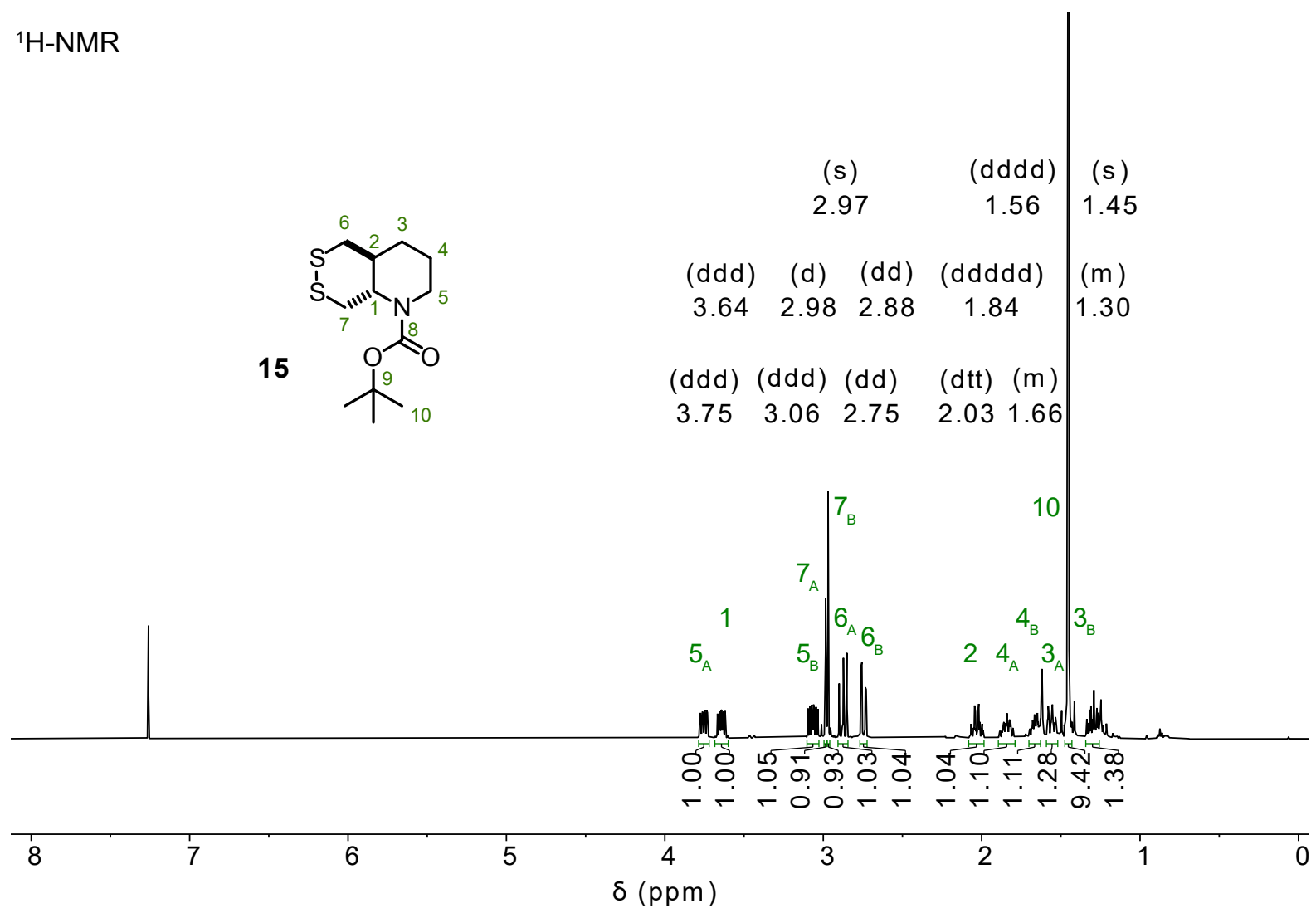

${ }^{13} \mathrm{C}-\mathrm{NMR}$
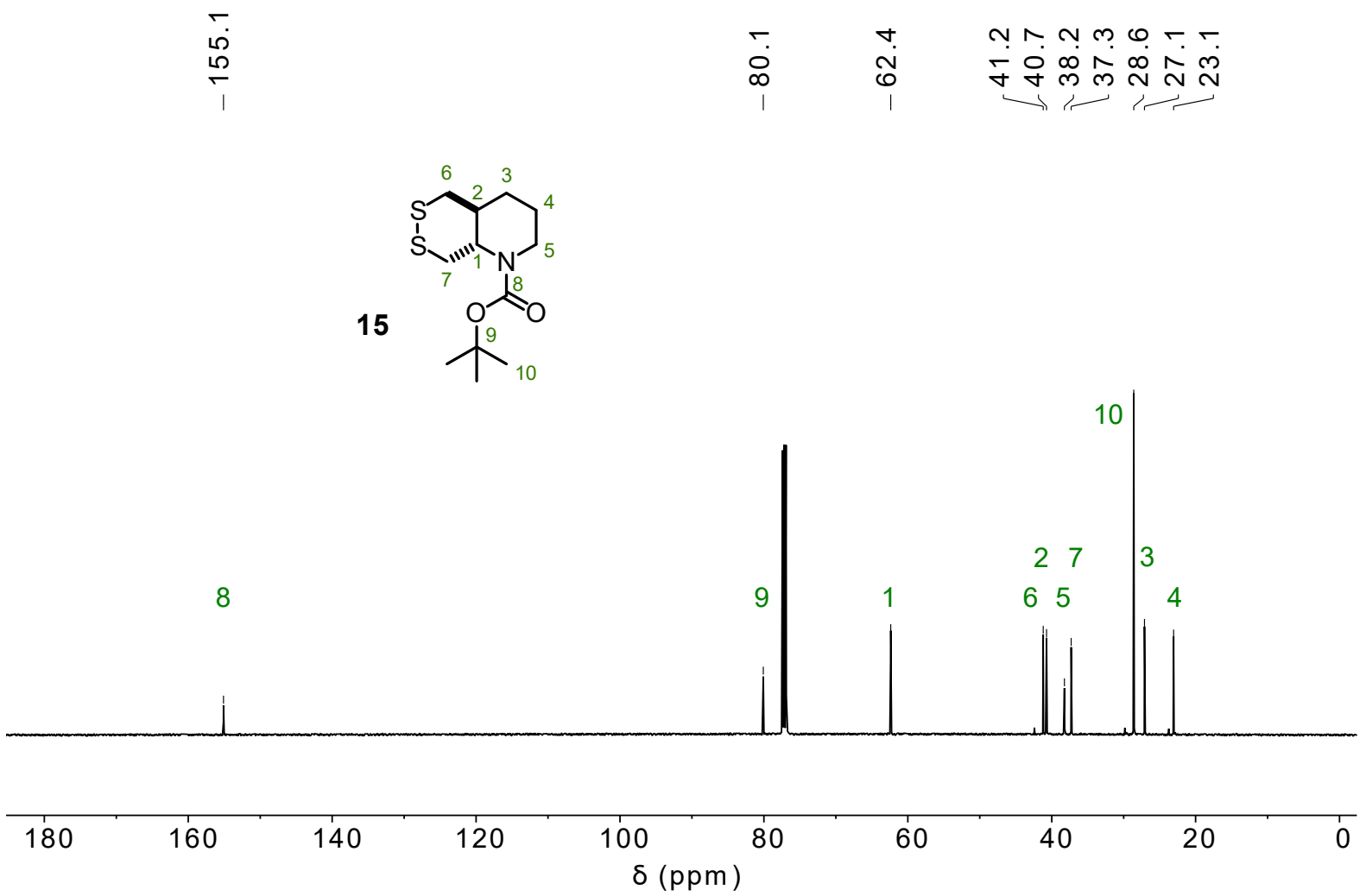
cis-octahydro-[1,2]dithiino[4,5-b]pyridine hydrochloride (18)

${ }^{1} \mathrm{H}-\mathrm{NMR}(373 \mathrm{~K})$

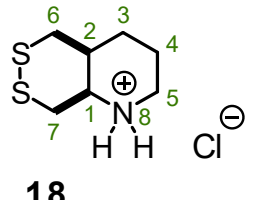

$$
\begin{array}{llll}
(d t) & \text { (d) } & \text { (s) } \quad \text { (td) }
\end{array}
$$

\begin{tabular}{l|ll}
3.663 .14 & 1.91 & 1.61
\end{tabular}

(dd) $(m) \quad(d d q)$

$3.512 .98 \quad 1.82$

(s)

(s)

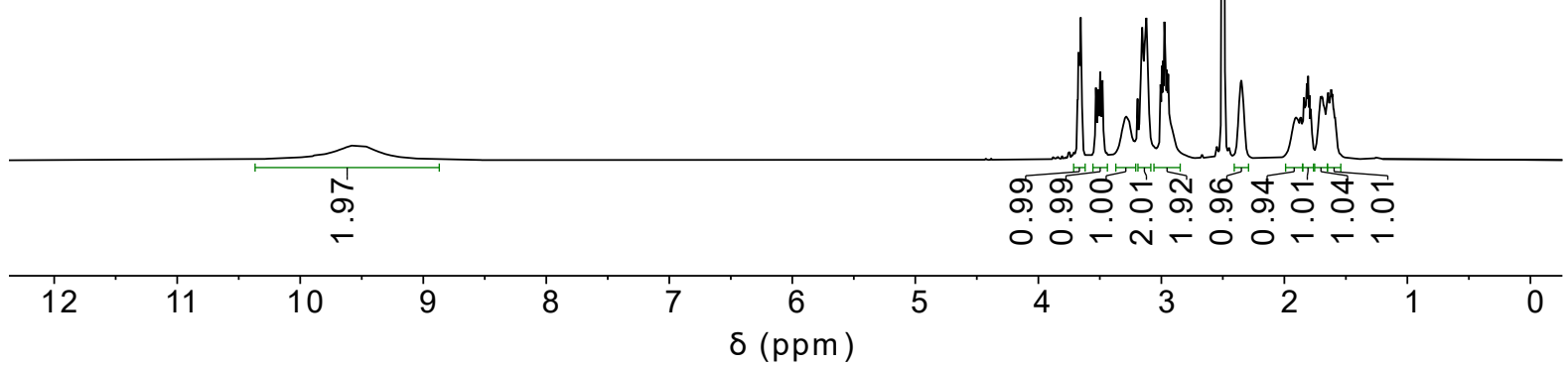


cis-octahydrofuro[3,4-b]pyridine hydrochloride (19)

${ }^{1} \mathrm{H}-\mathrm{NMR}$

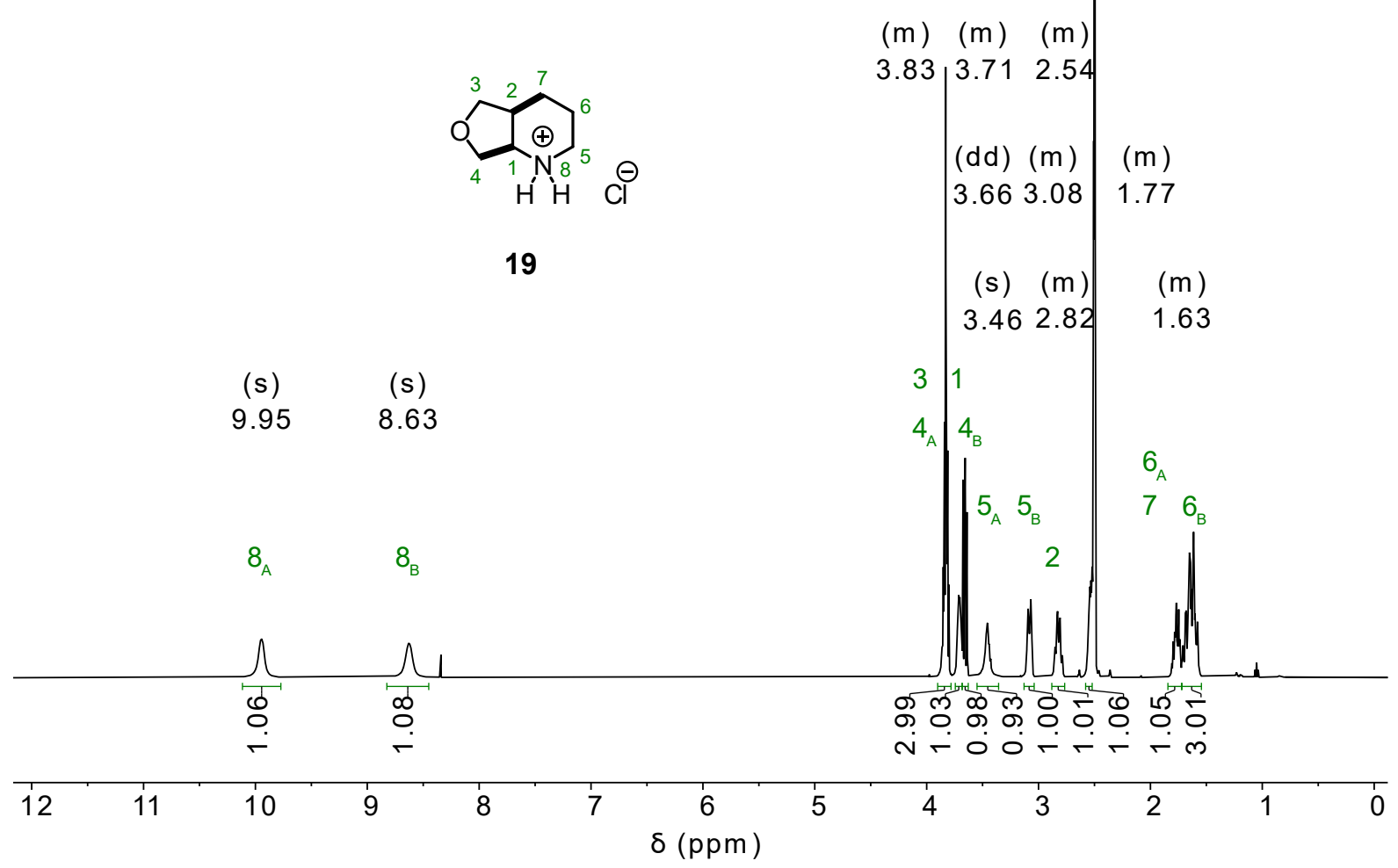

${ }^{13} \mathrm{C}-\mathrm{NMR}$

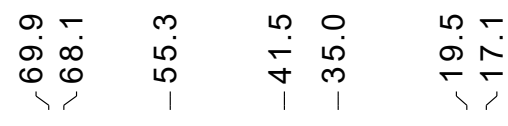

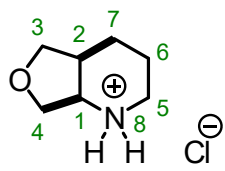

19

\begin{tabular}{ccccccccc}
\hline 180 & 160 & 140 & 120 & 100 & 80 & 60 & 40 & 20 \\
$\delta(\mathrm{ppm})$
\end{tabular}


(S)-N-Boc-N-methyl-1,2-dithian-4-amine (24)

${ }^{1} \mathrm{H}-\mathrm{NMR}$

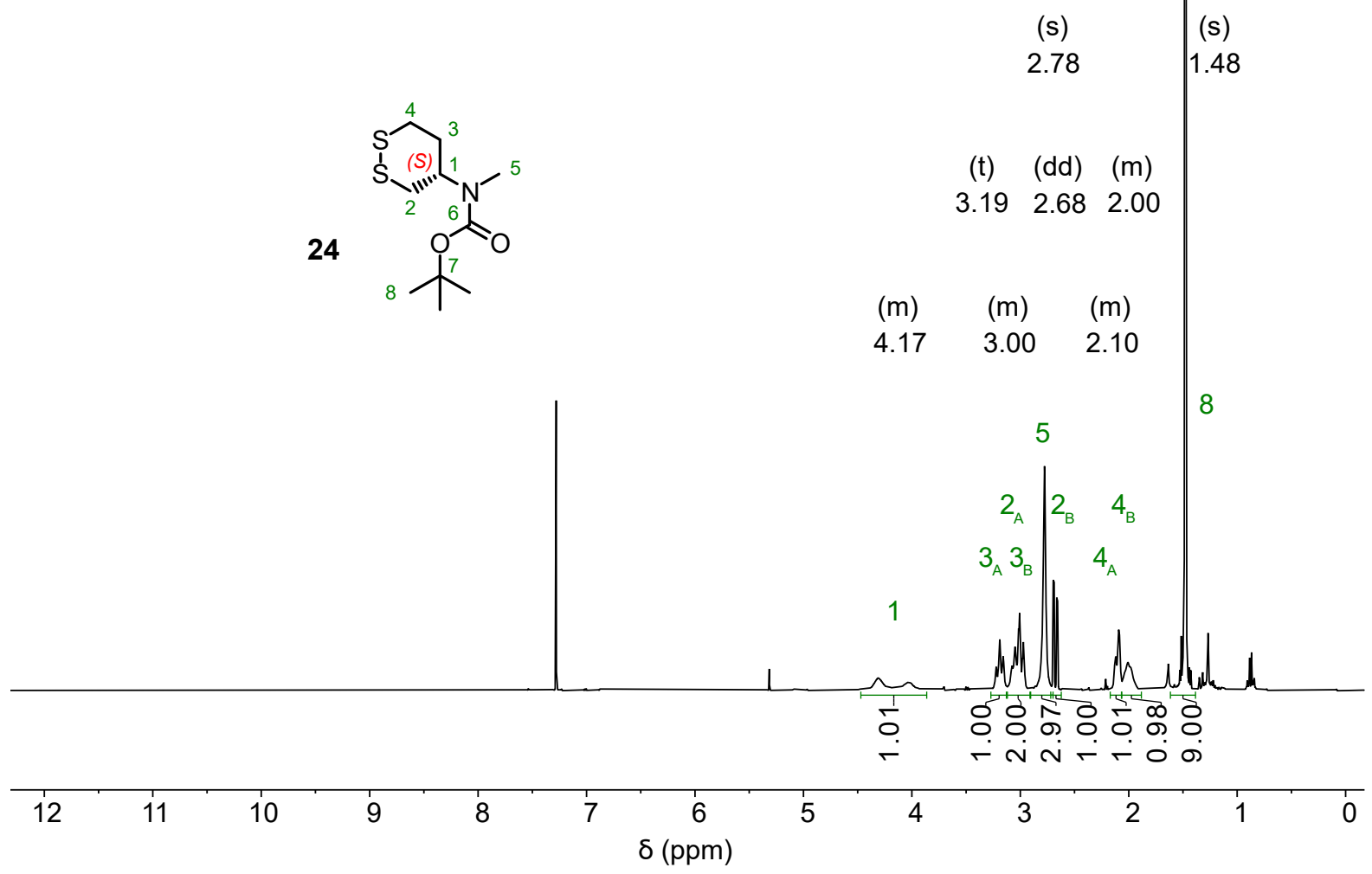

${ }^{13} \mathrm{C}-\mathrm{NMR}$

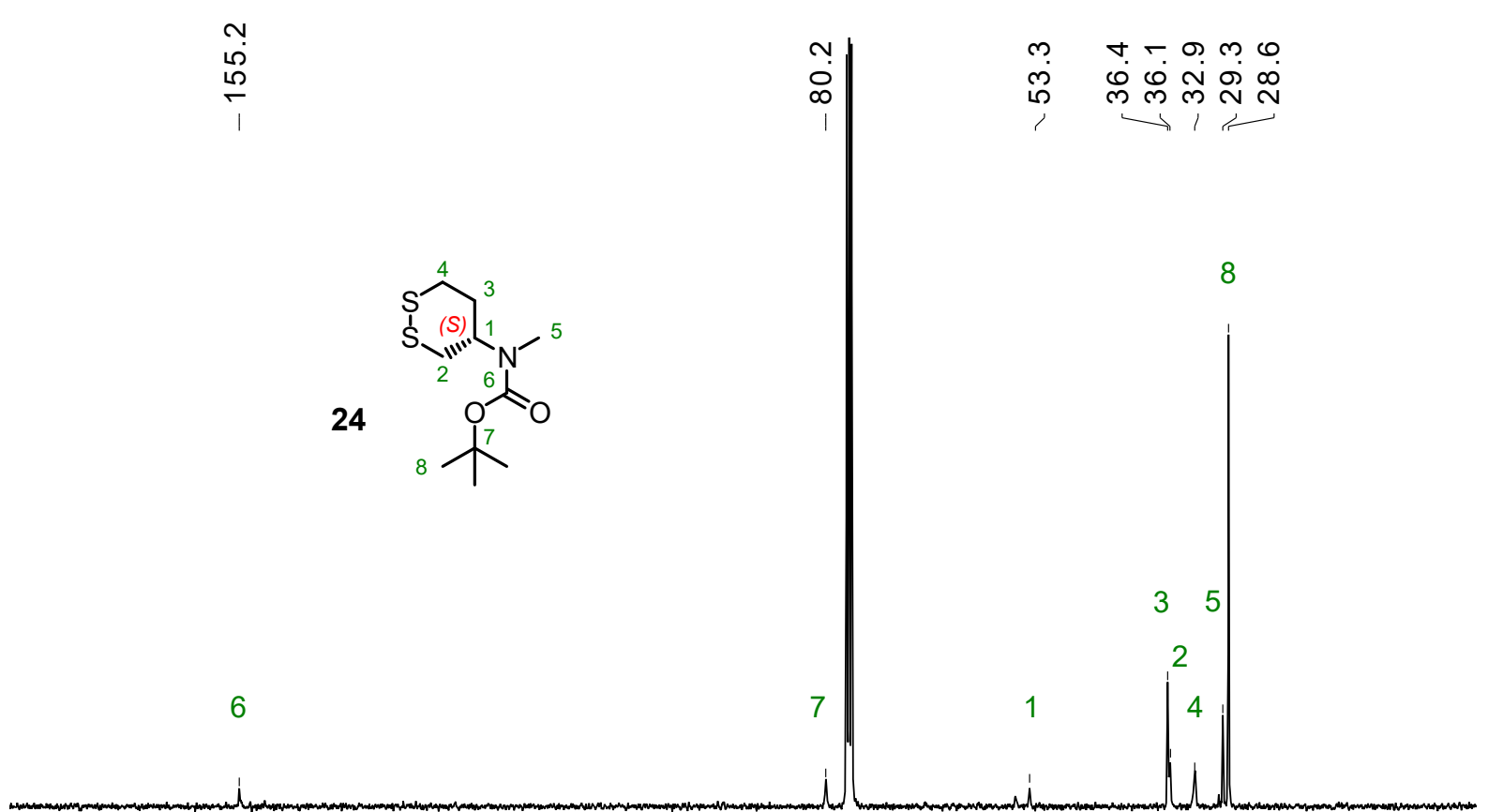

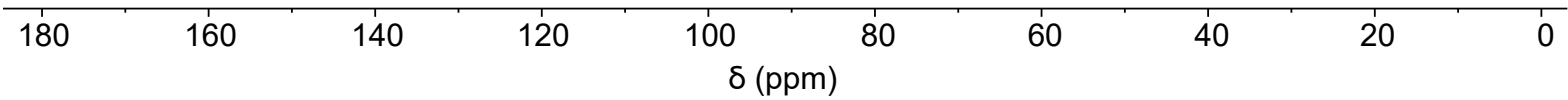


(S)-N-methyl-1,2-dithian-4-amine hydrochloride (25)

${ }^{1} \mathrm{H}-\mathrm{NMR}$

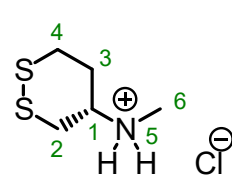

(s)

2.53

25

(s)

9.34

(m) (m)

3.292 .97

(q)

(m)

$3.16 \quad 2.42$

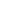

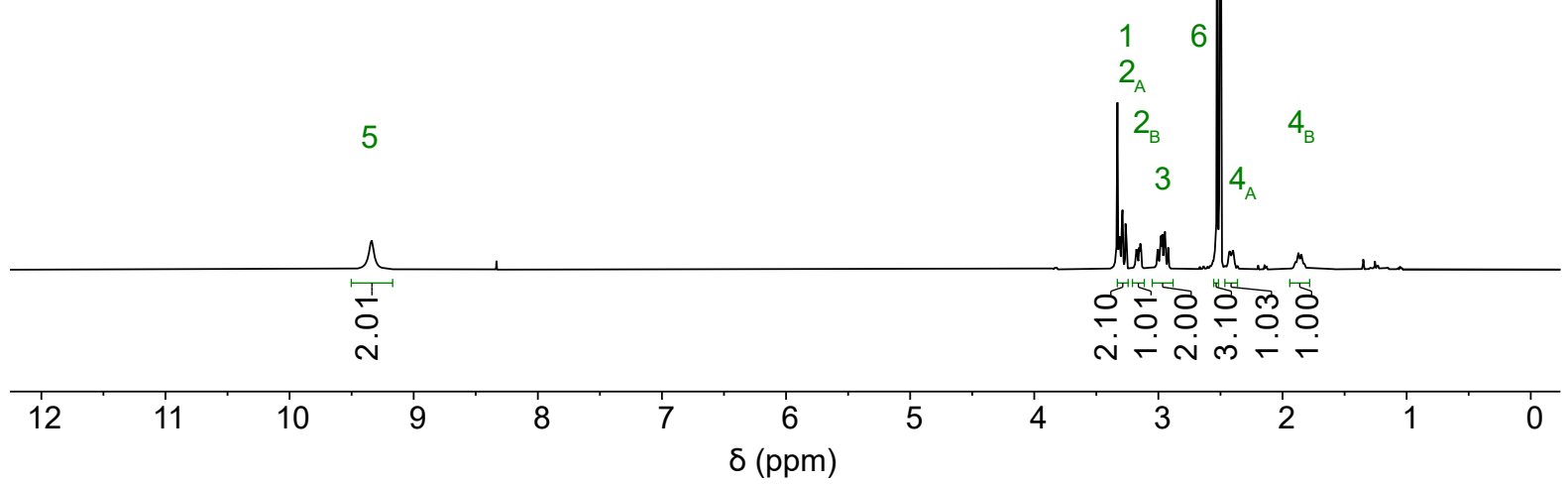

${ }^{13} \mathrm{C}-\mathrm{NMR}$

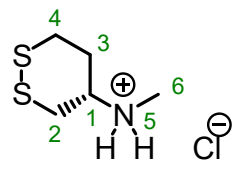

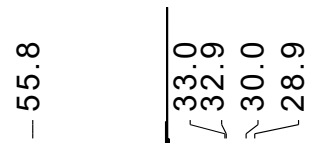

25

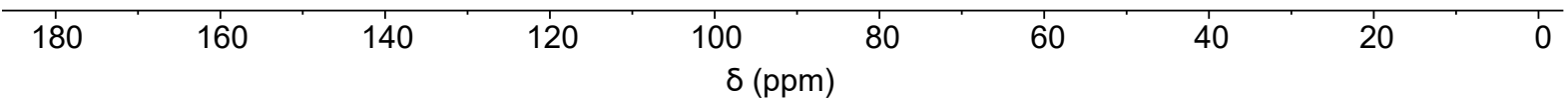


1,2-dithian-4-ol (30)

${ }^{1} \mathrm{H}-\mathrm{NMR}$

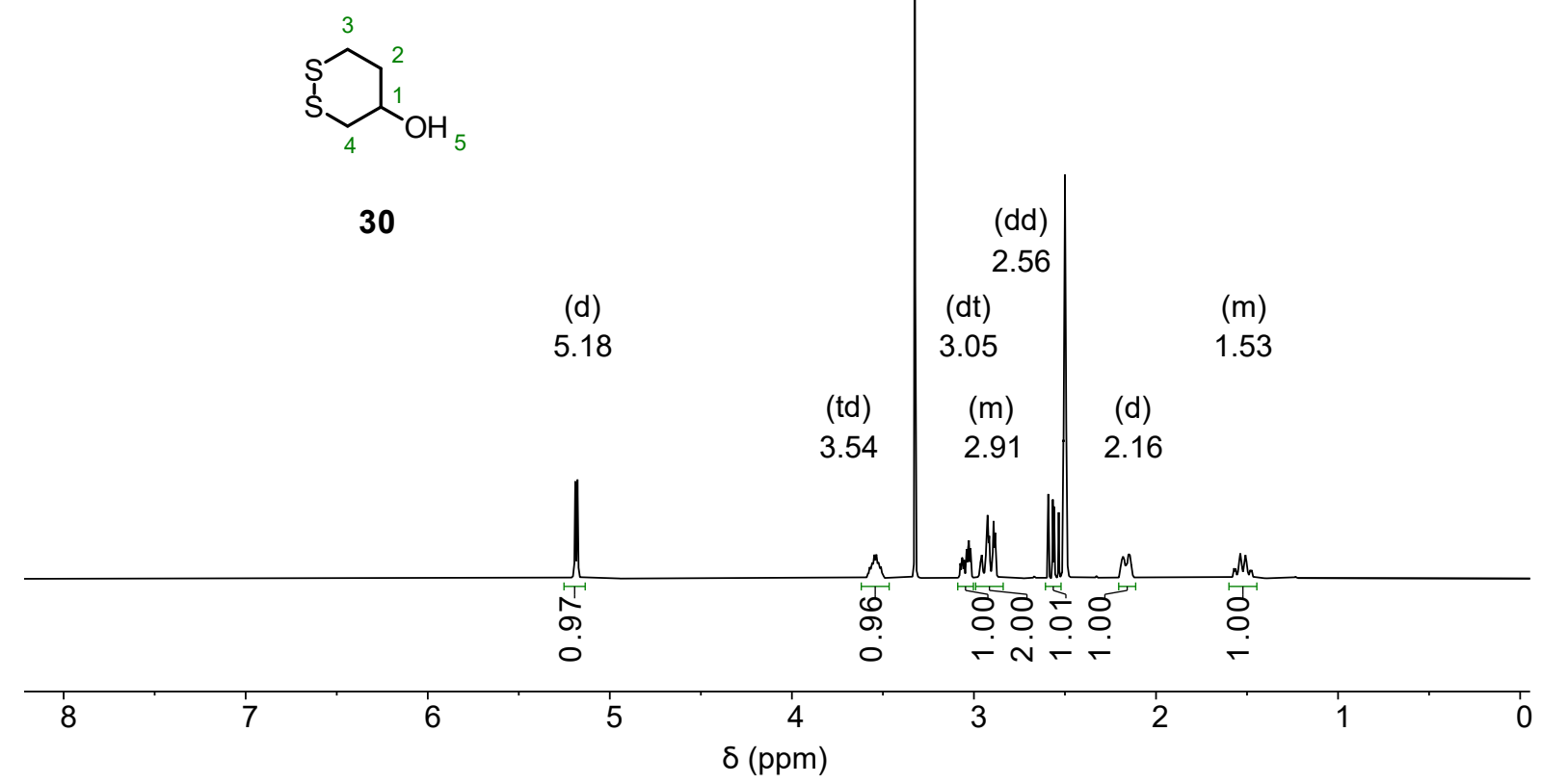

${ }^{13} \mathrm{C}-\mathrm{NMR}$

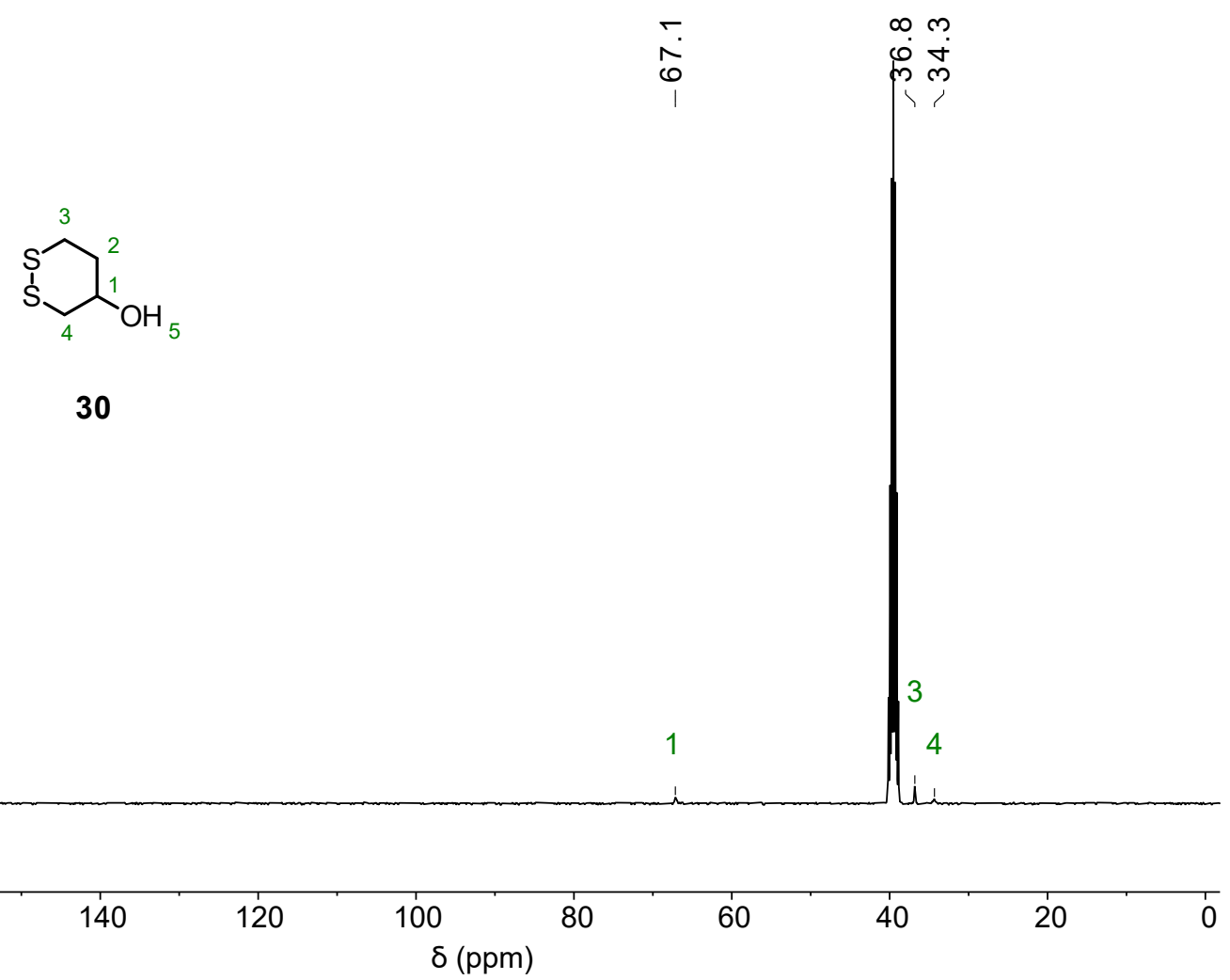


$N$-Boc, $N$ '-Boc 2,2'-disulfanediylbis( $N$-methylethan-1-amine) (33)

${ }^{1} \mathrm{H}-\mathrm{NMR}$

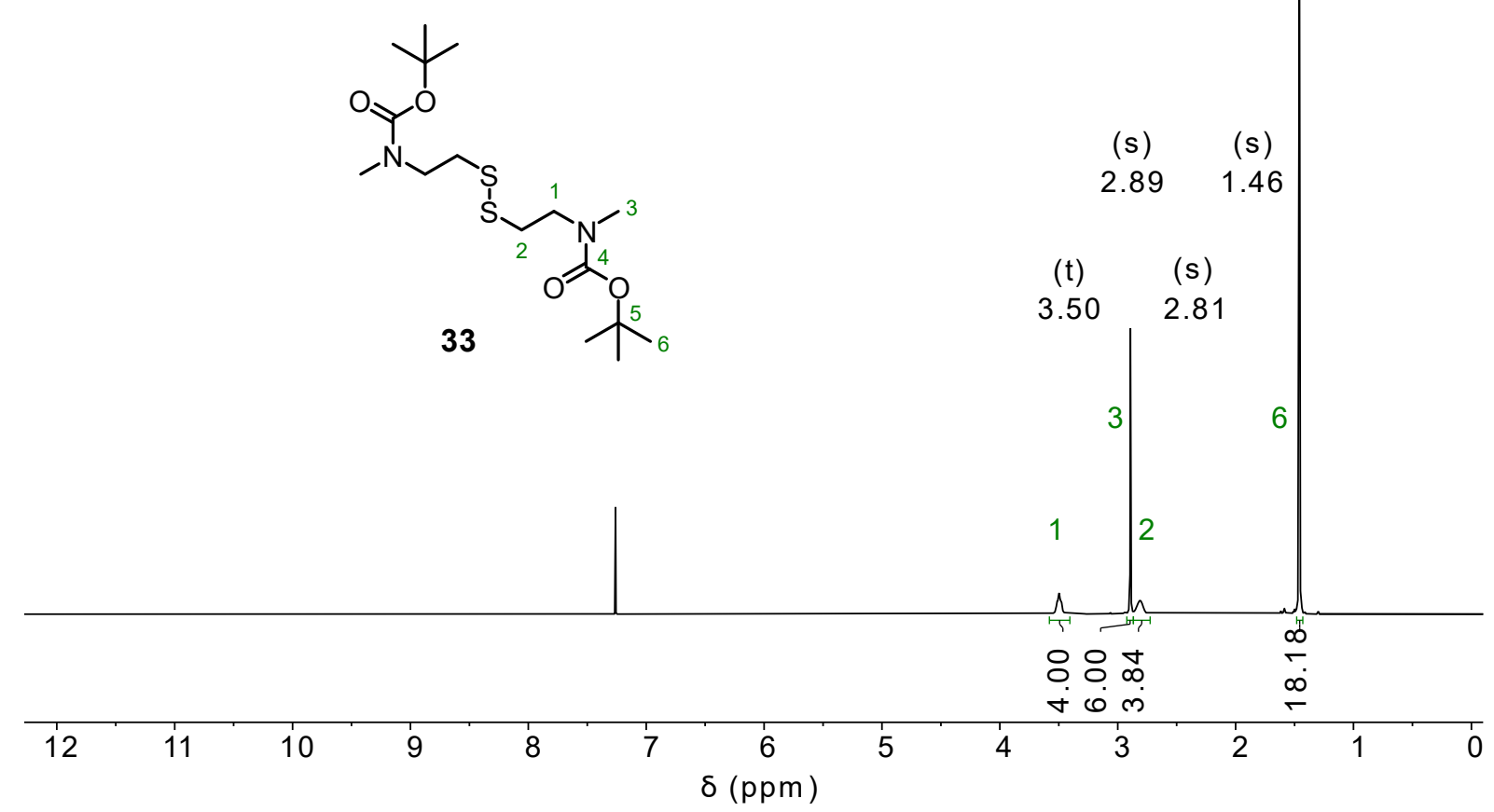

${ }^{13} \mathrm{C}-\mathrm{NMR}$

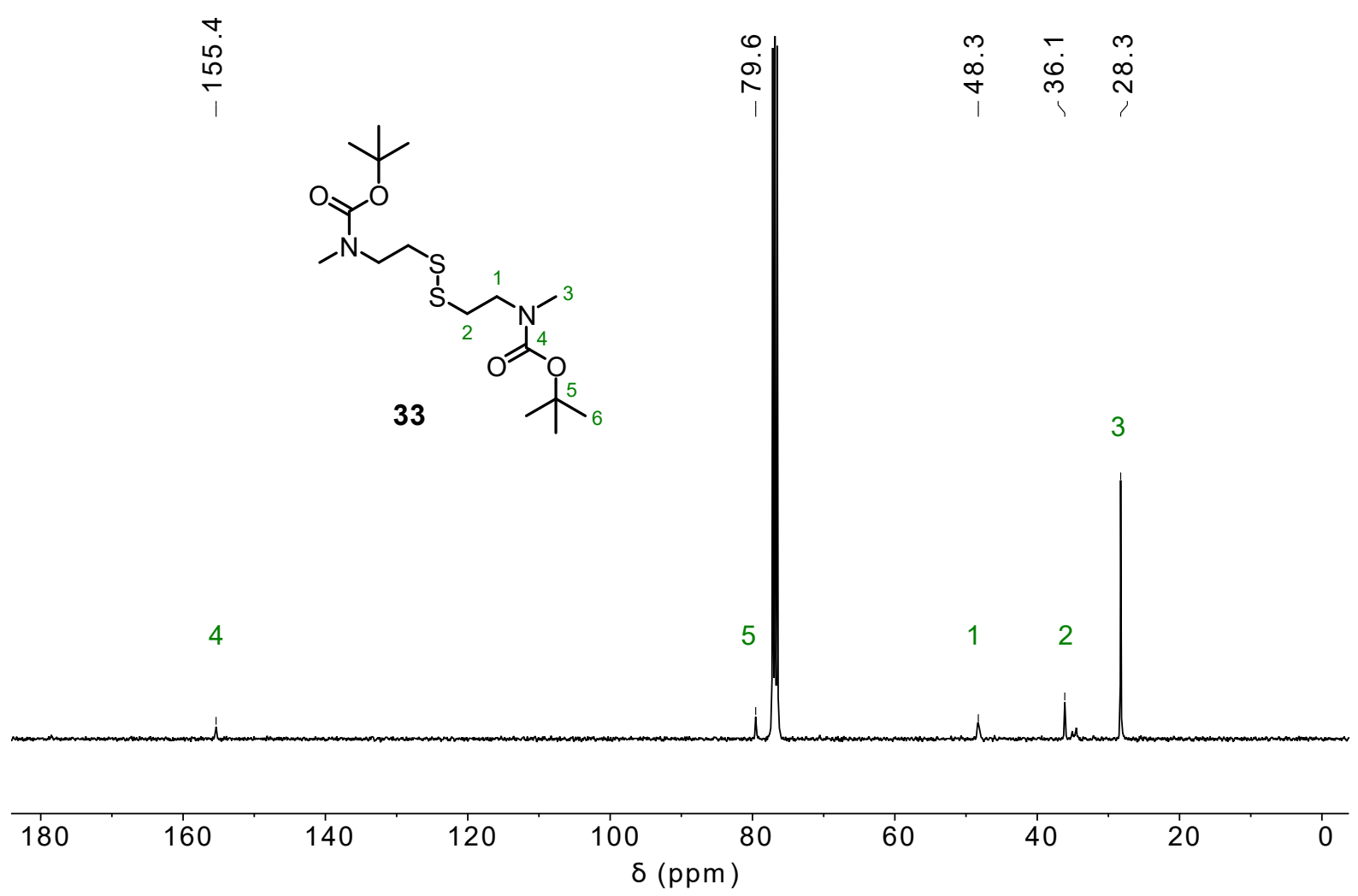


2,2'-disulfanediylbis( $N$-methylethan-1-amine) dihydrochloride (34)

${ }^{1} \mathrm{H}-\mathrm{NMR}$

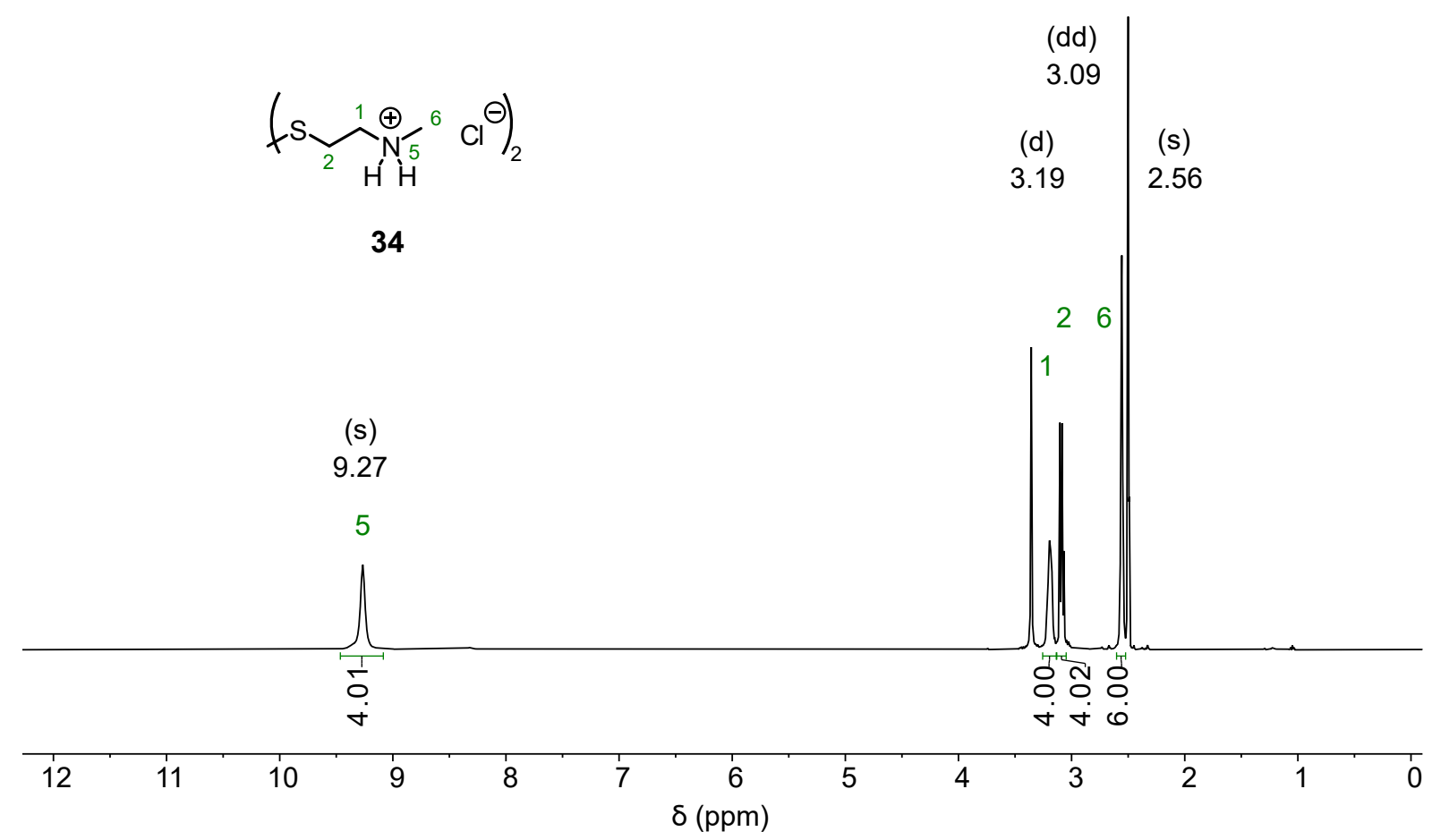

${ }^{13} \mathrm{C}-\mathrm{NMR}$

$\underbrace{\circ}_{i} \quad \stackrel{n}{\sim}$

$(s \underbrace{1}_{2} \stackrel{\oplus \mathrm{H}^{\prime} \mathrm{H}}{N_{5}^{6}}{ }^{6} \mathrm{Cl}^{\Theta})_{2}$

34

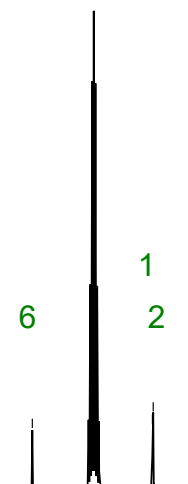

180

160

140

120

100

$\delta(\mathrm{ppm})$

60

40

20 
$N$-methyl-2-((2-morpholinoethyl)disulfaneyl)ethan-1-amine dihydrochloride (40)

${ }^{1} \mathrm{H}-\mathrm{NMR}$

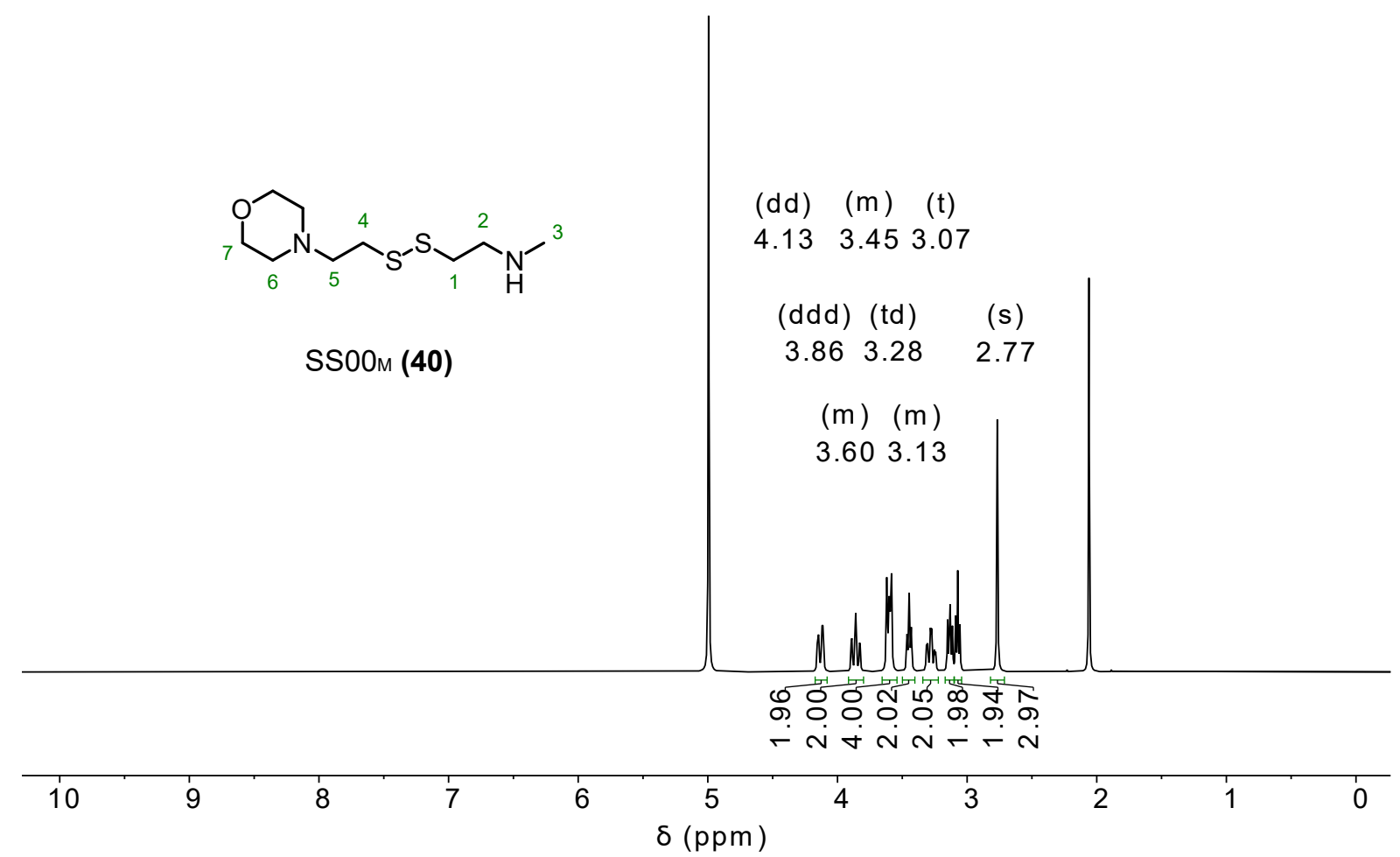

${ }^{13} \mathrm{C}-\mathrm{NMR}$
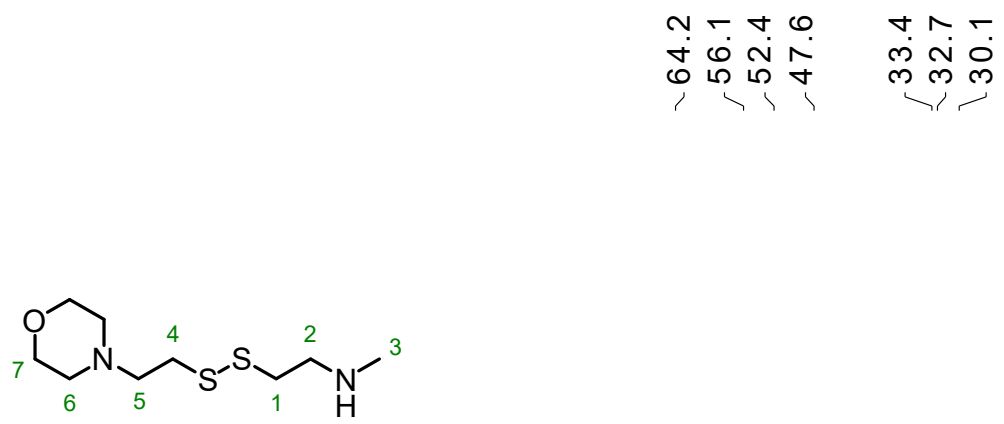

$\mathrm{SSOOM} \mathrm{(40)}$

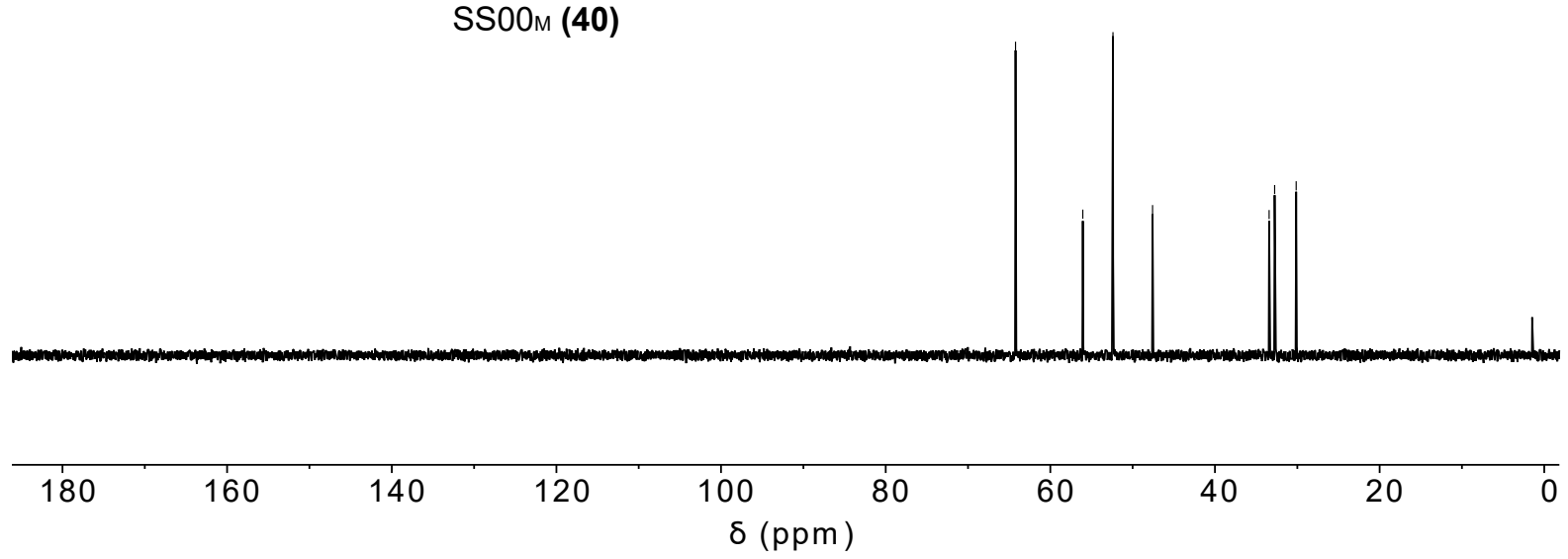


${ }^{1} \mathrm{H}-\mathrm{NMR}$

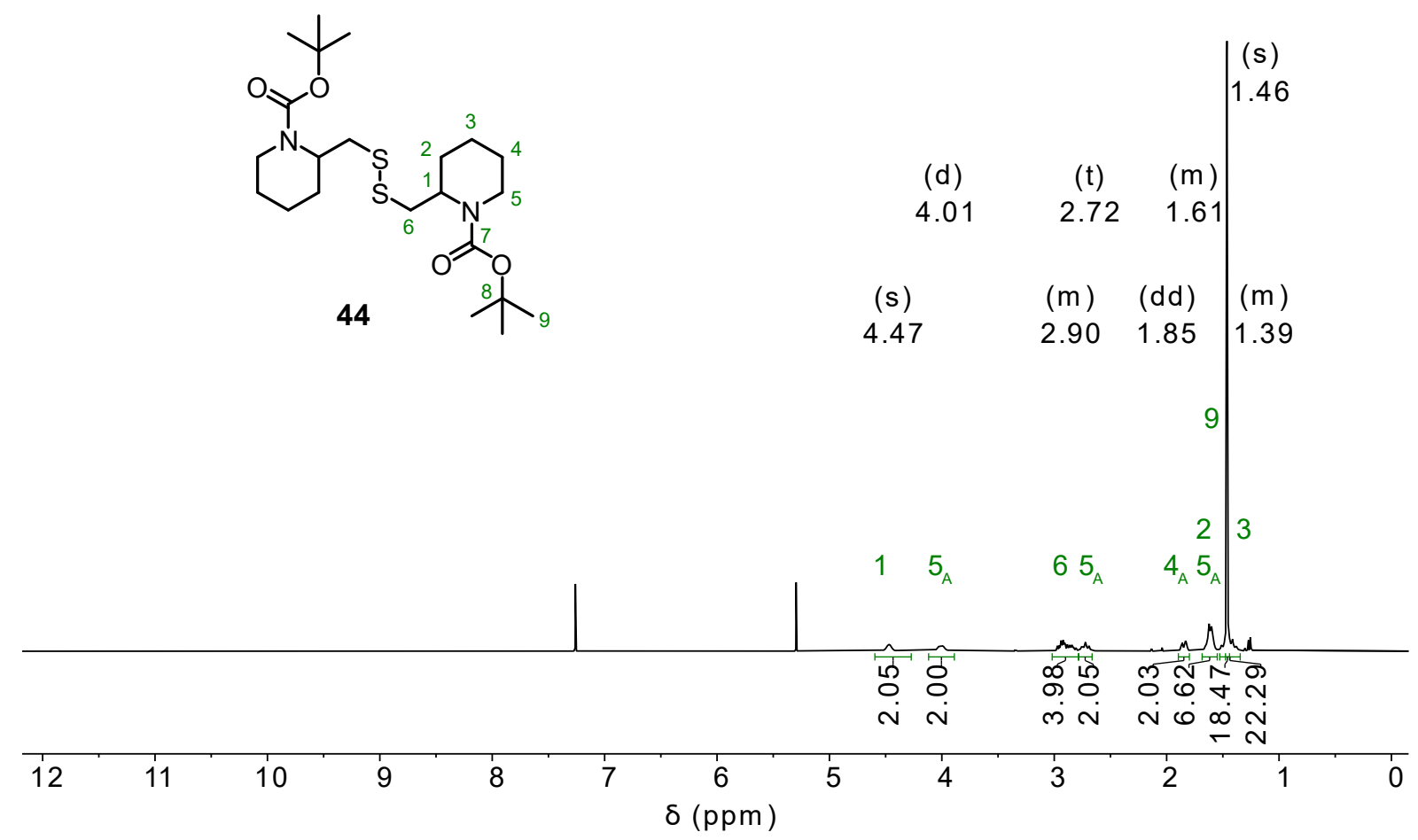

${ }^{13} \mathrm{C}-\mathrm{NMR}$

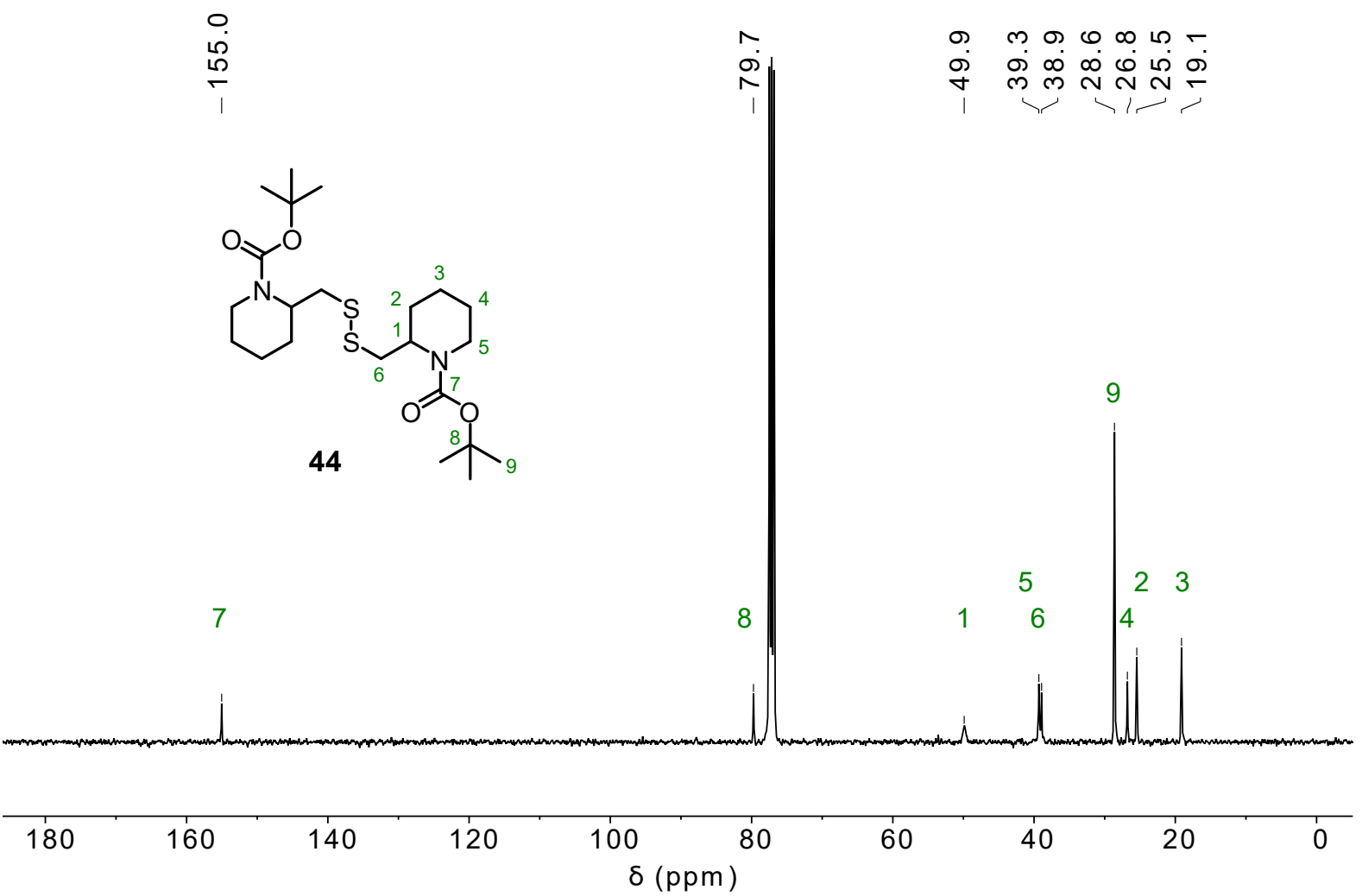

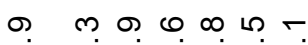

क $\infty \infty \infty i \infty$ 
1,2-bis(piperidin-2-ylmethyl)disulfane dihydrochloride (45)

${ }^{1} \mathrm{H}-\mathrm{NMR}$

$$
(\mathrm{S} \underbrace{1}_{7} \overbrace{\mathrm{H}^{\prime} \mathrm{H}}^{\oplus} \overbrace{6}^{4} \mathrm{Cl}^{\Theta})_{2}
$$

45

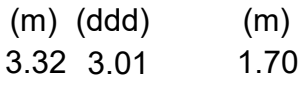

(m) (d) (d) (m)

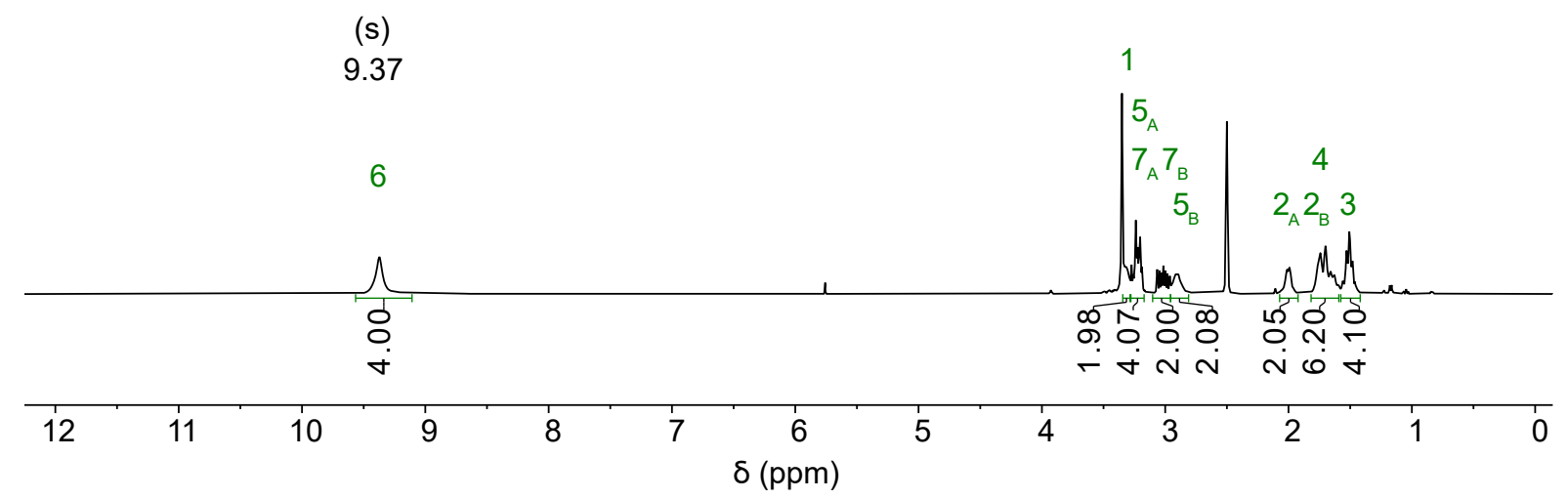

${ }^{13} \mathrm{C}-\mathrm{NMR}$

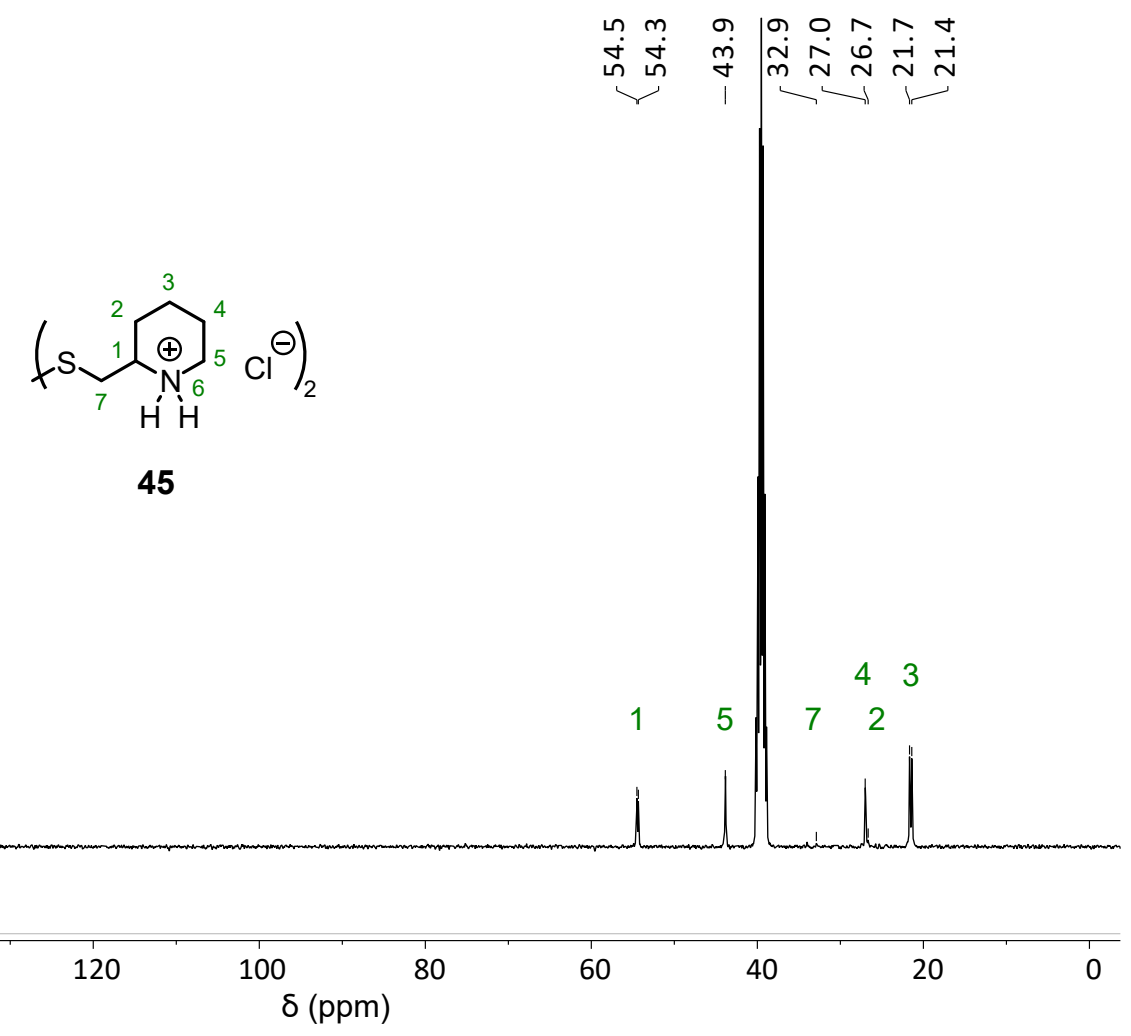


${ }^{1} \mathrm{H}-\mathrm{NMR}$

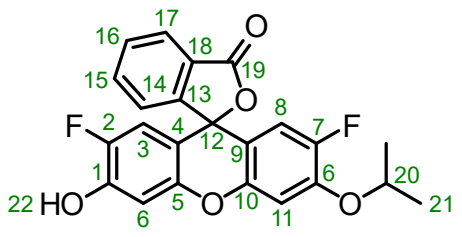

62

$\begin{array}{ccc}(d) & (d) & (d) \\ 8.00 & 7.30 & 6.51\end{array}$

(t) $\quad(d) \quad(d)$

$\begin{array}{lll}7.80 & 7.20 \quad 6.55\end{array}$

(s)

10.77

(t) (d)

$7.74 \quad 6.90$

(p)

$\begin{array}{llll}15 & 14 & 3 & 8\end{array}$

$\begin{array}{llll}17 & 16 & 6 & 11\end{array}$

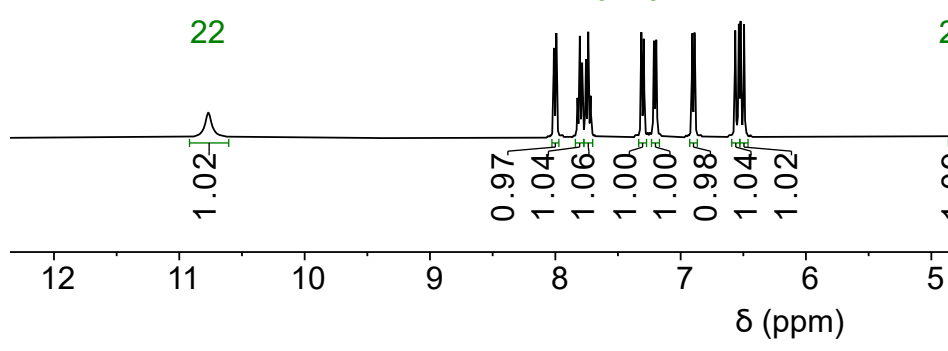

(dd)

${ }^{13} \mathrm{C}-\mathrm{NMR}$

ก 穴 $\infty \circ$ ?

$\infty \sim$

$\sim \sim N$

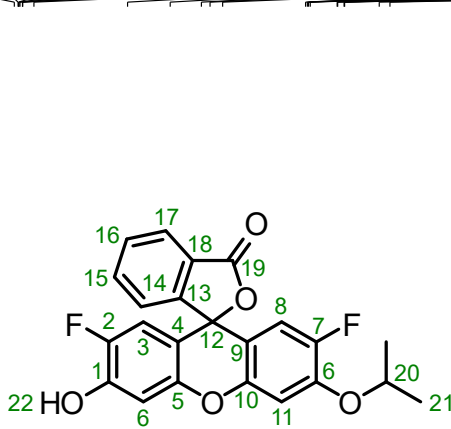

62

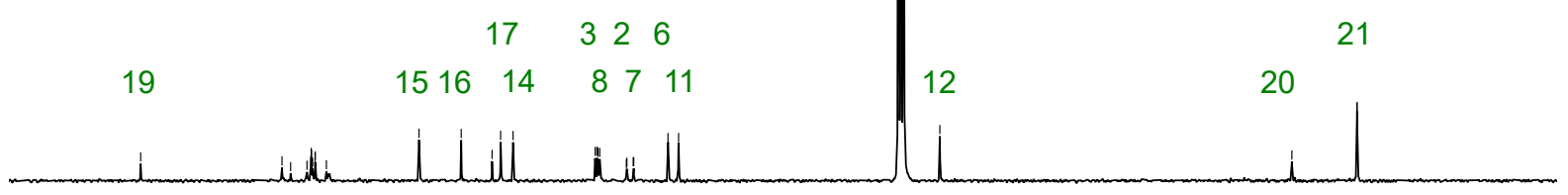

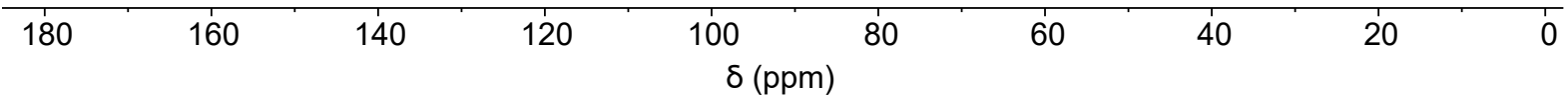


trans-(hexahydro-[1,2]dithiino[4,5-b]pyridin-1(2H)-yl)benzamide (SS66T-Bz, 65)

${ }^{1} \mathrm{H}-\mathrm{NMR}$

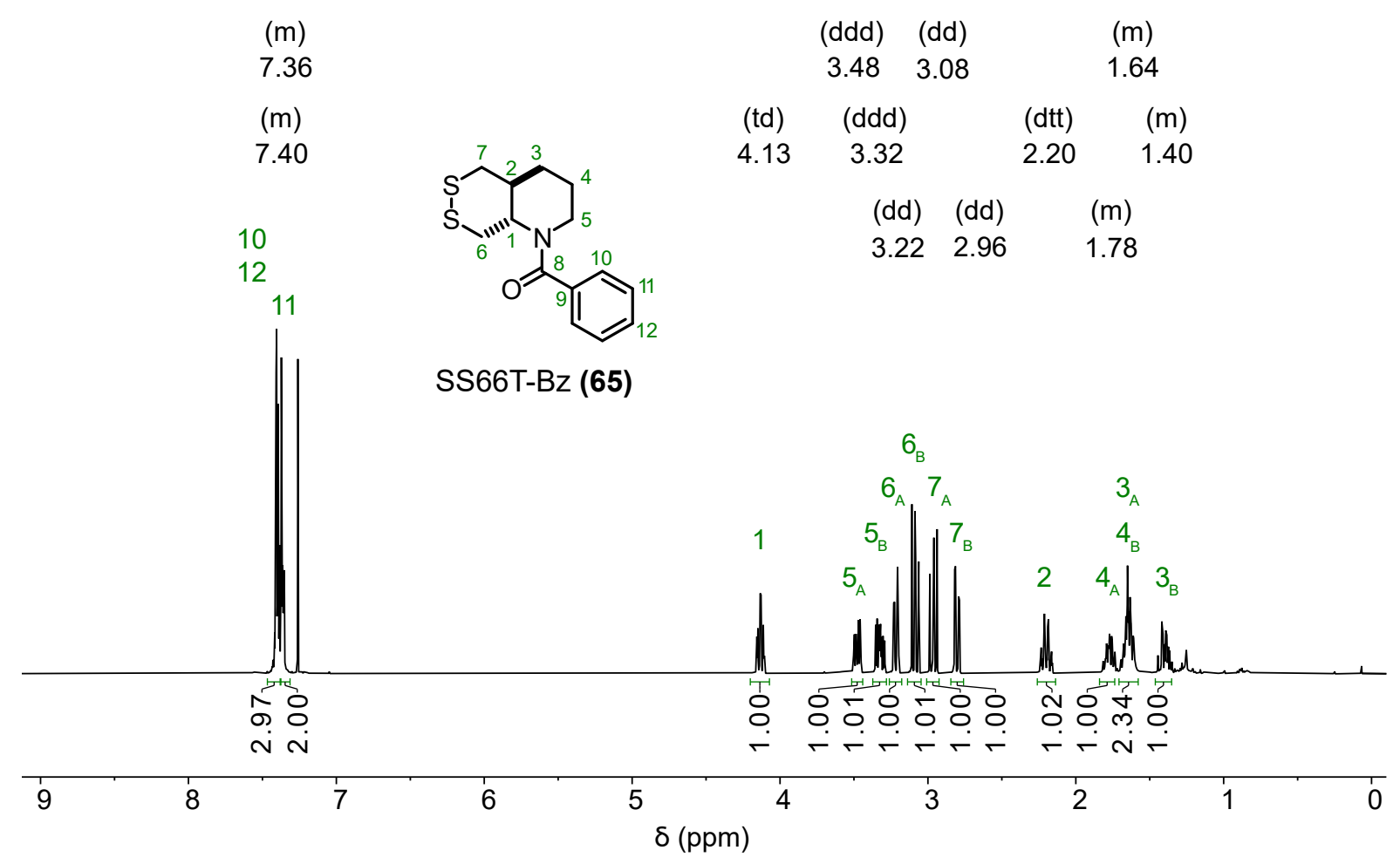

${ }^{13} \mathrm{C}-\mathrm{NMR}$

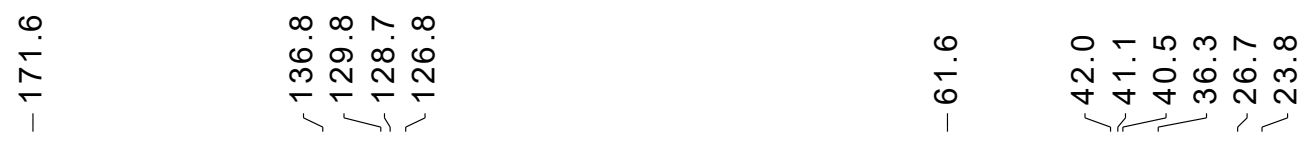

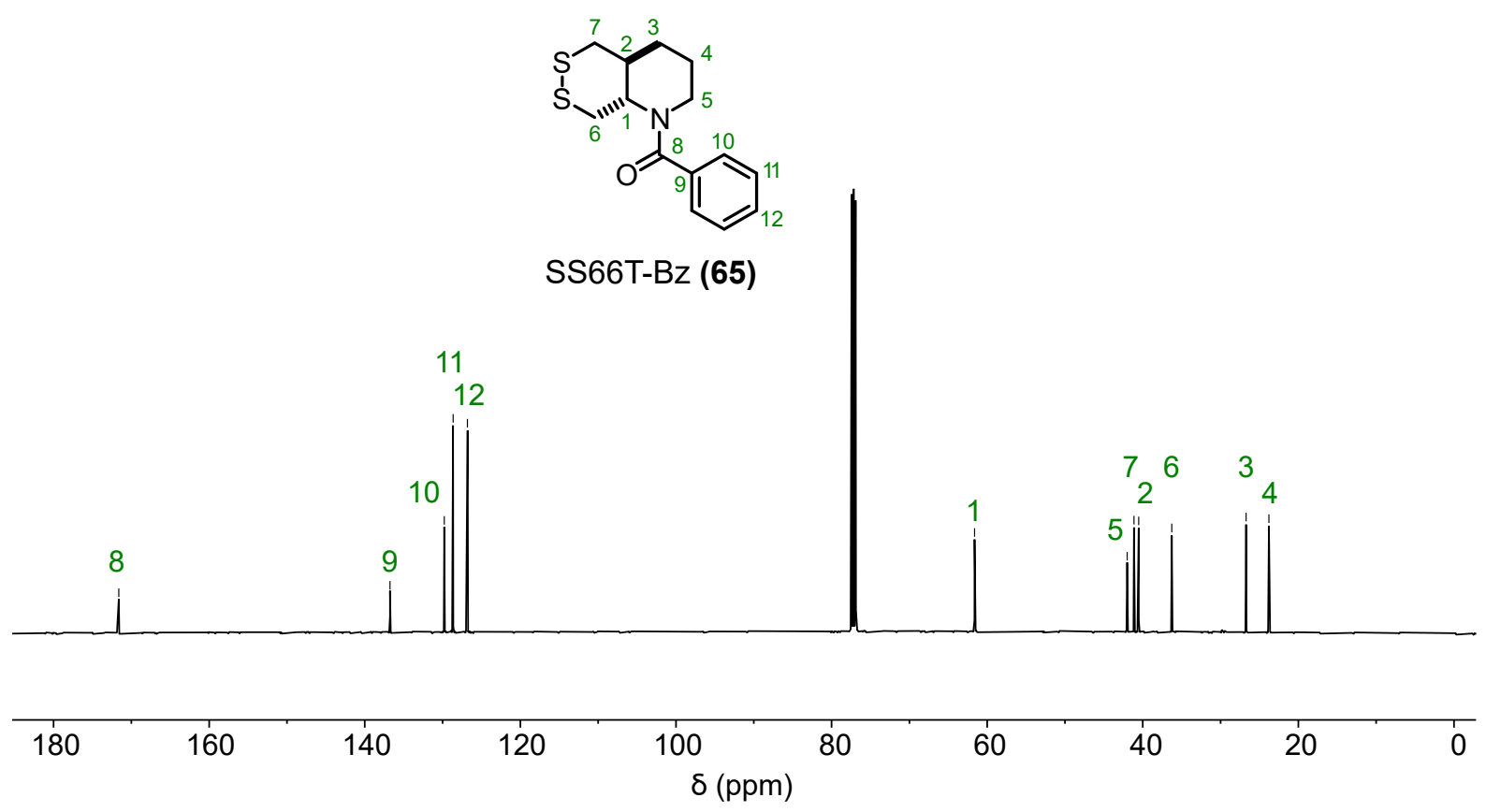


cis-(hexahydro-[1,2]dithiino[4,5-b]pyridin-1(2H)-yl)benzamide (SS66C-Bz, 66)

${ }^{1} \mathrm{H}-\mathrm{NMR}$

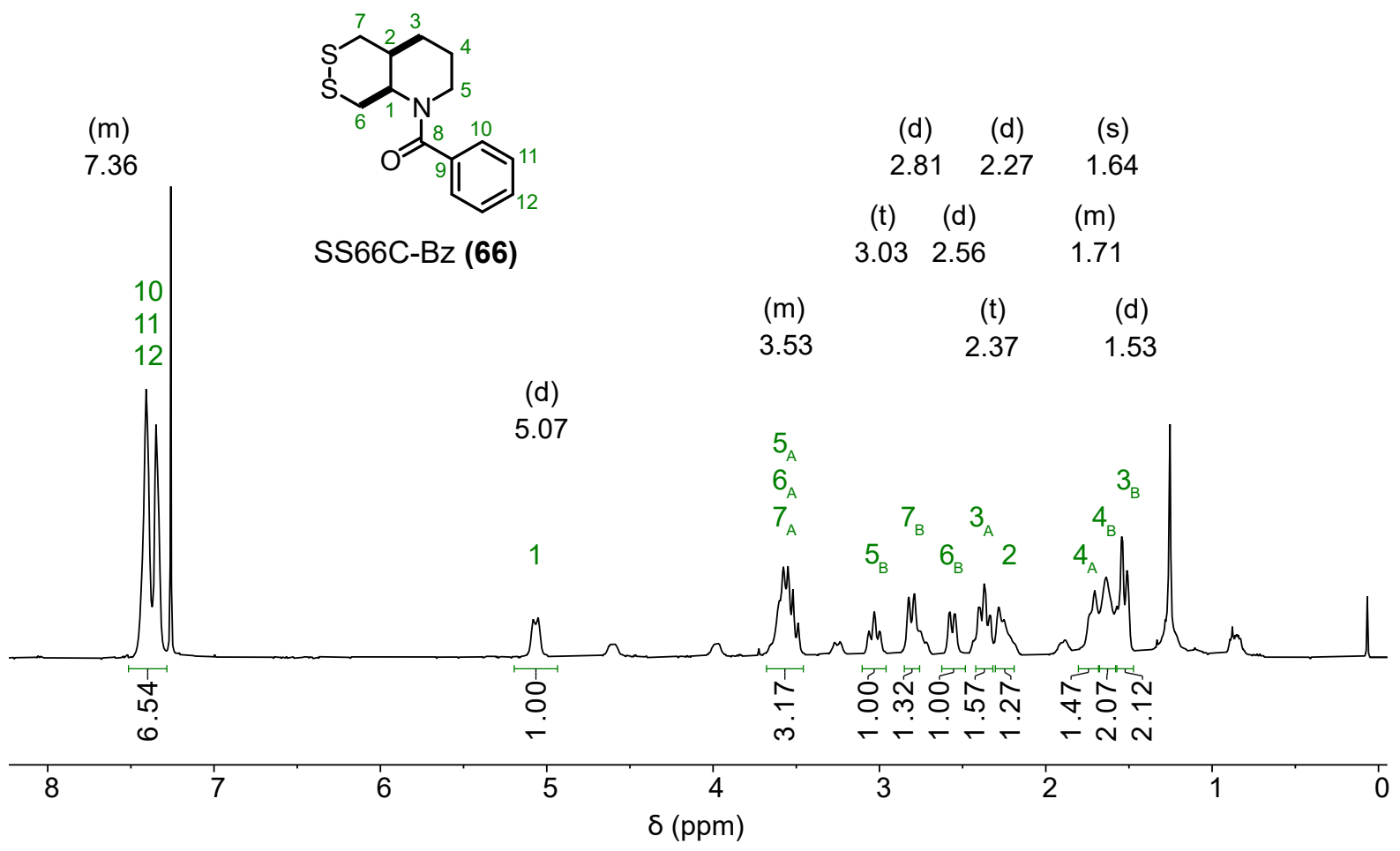

${ }^{13} \mathrm{C}-\mathrm{NMR}$

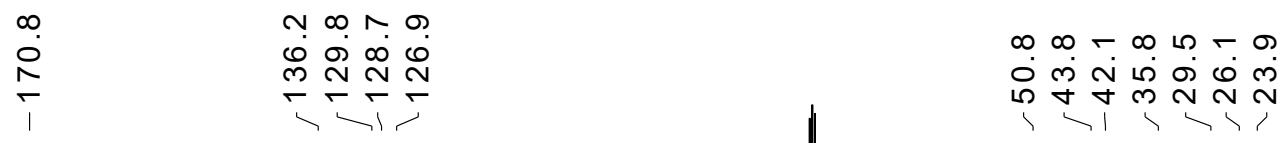

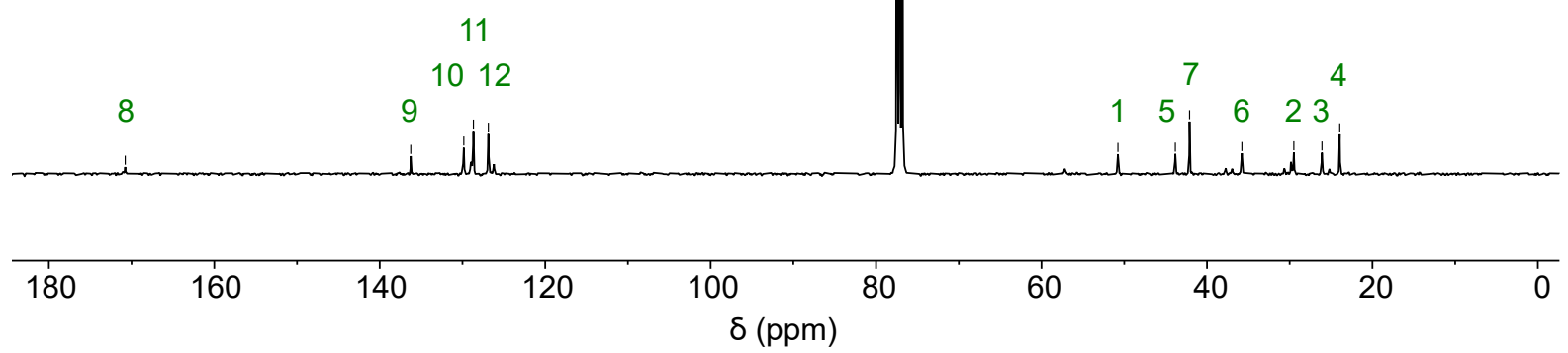


(S)-N-methyl-N-(1,2-dithian-4-yl)benzamide (SS60-Bz, 67)

${ }^{1} \mathrm{H}-\mathrm{NMR}(373 \mathrm{~K})$

(dd) (m)

3.192 .87

(m)

(dt) (s)

7.36

$3.11 \quad 2.84$

(m)

7.44

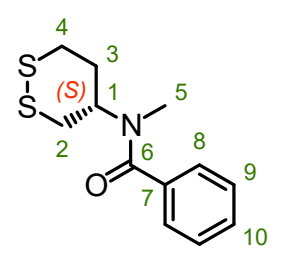

(s)

(d) (m)

4.19

$3.02 \quad 2.13$

SS60-Bz (67)

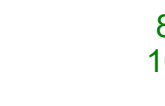

8
10

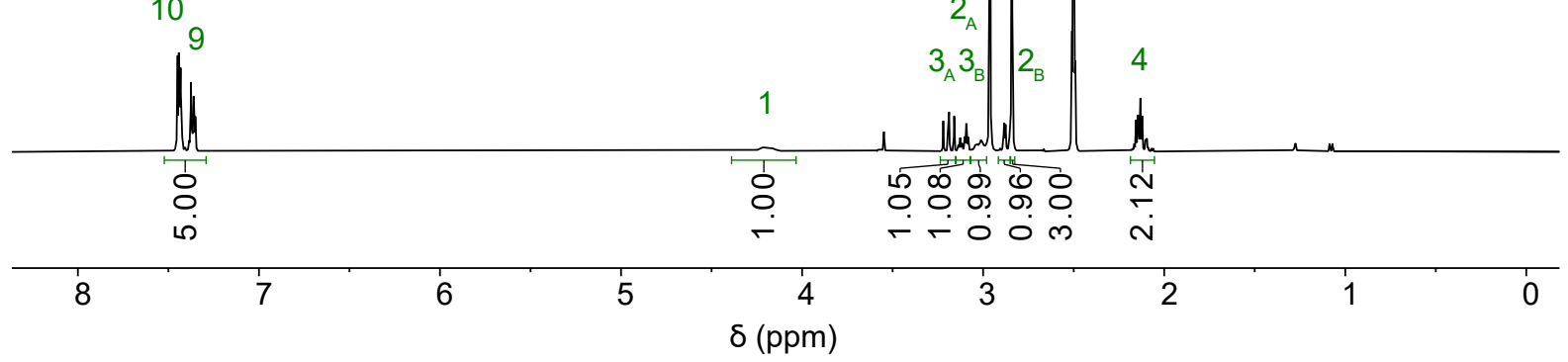

${ }^{13} \mathrm{C}-\mathrm{NMR}(373 \mathrm{~K})$

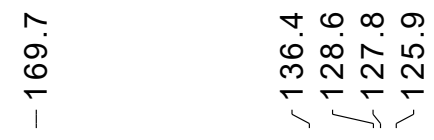

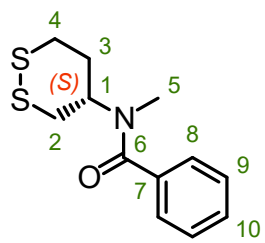

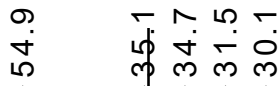

SS60-Bz (67)

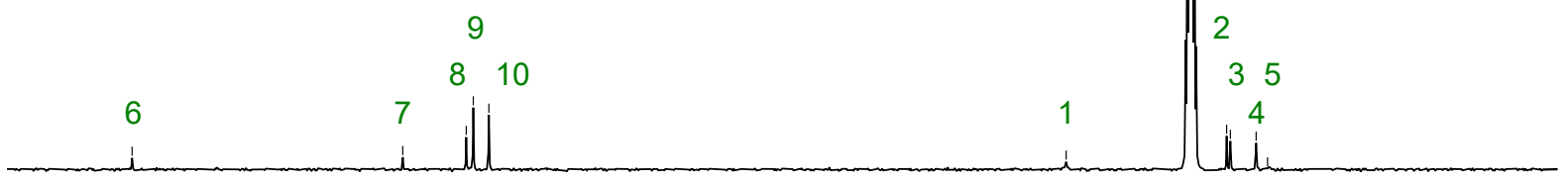

180

160

140

120

100

80

60

$\delta(p p m)$

20

0 
$N$-methyl-N-(1,2-dithiolan-4-yl)benzamide (SS50-Bz, 68)

${ }^{1} \mathrm{H}-\mathrm{NMR}(373 \mathrm{~K})$

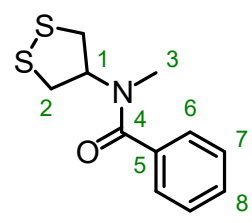

SS50-Bz (68) (m)

7.40

(m)

7.45

6
8

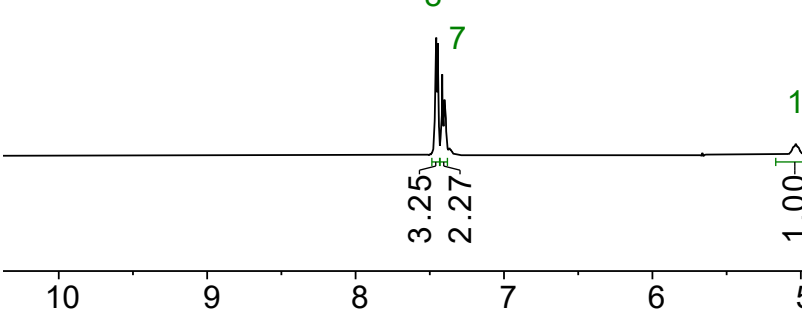

$\delta(\mathrm{ppm})$ (s)

2.92

(dd)

3.28

(s)

5.03

(dd)

3.37

$2_{B}$

$2_{\mathrm{A}}$

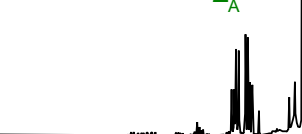

造客

${ }^{13} \mathrm{C}-\mathrm{NMR}(373 \mathrm{~K})$

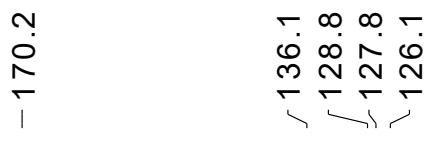

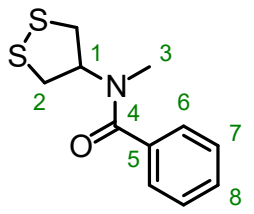

SS-50-Bz (68)

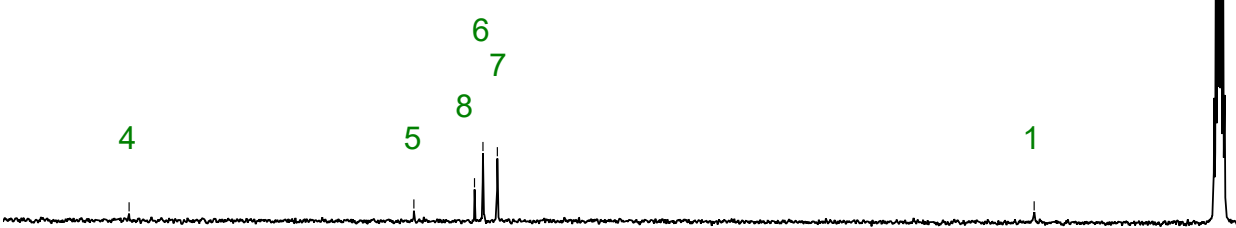

23 
(1,2,5-dithiazepan-5-yl)(phenyl)methanone (SS7-Bz, 69)

${ }^{1} \mathrm{H}-\mathrm{NMR}$

(s)

7.32

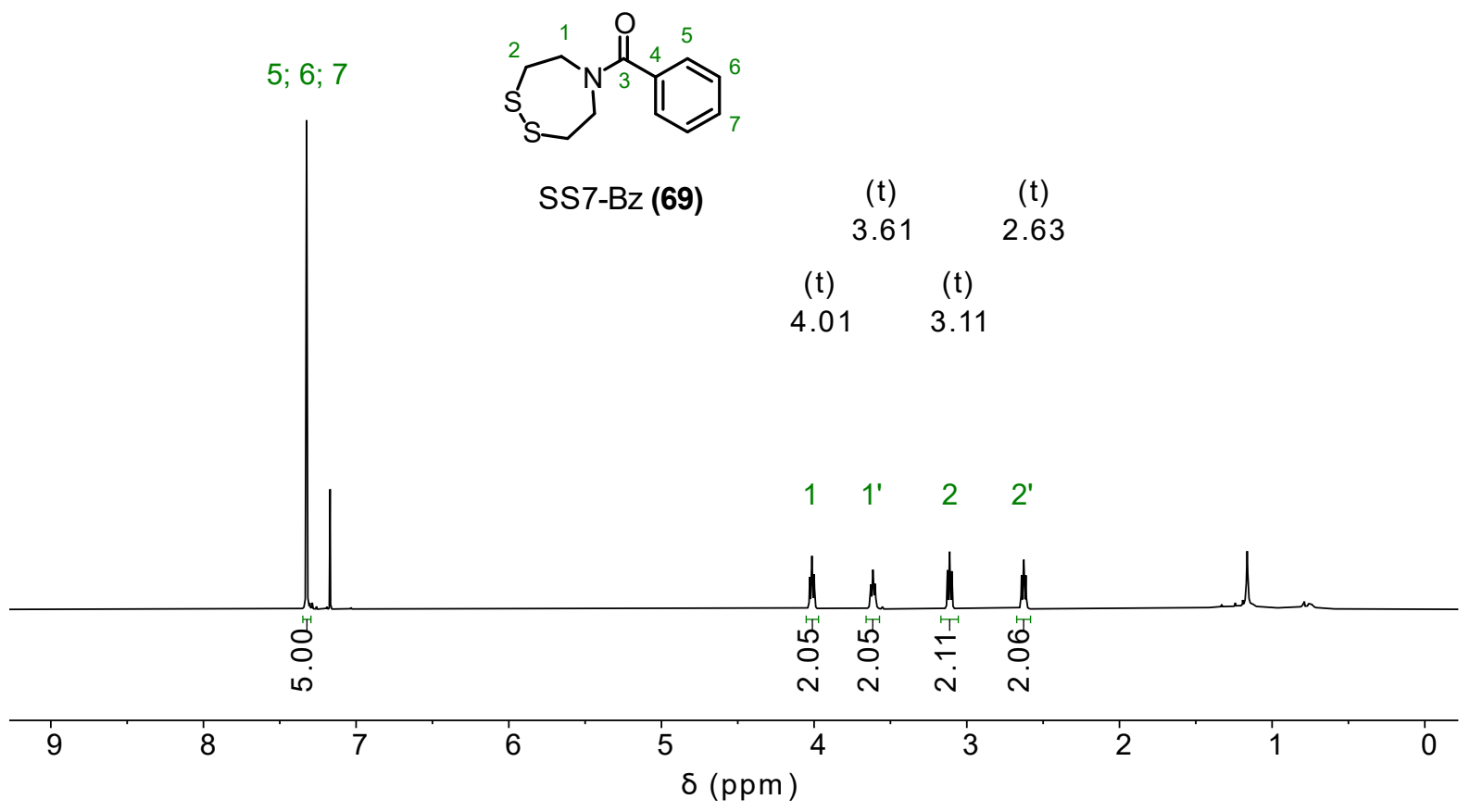

${ }^{13} \mathrm{C}-\mathrm{NMR}$

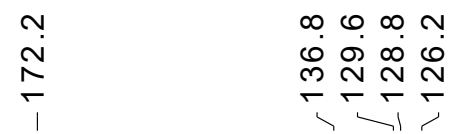

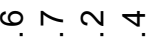

गु

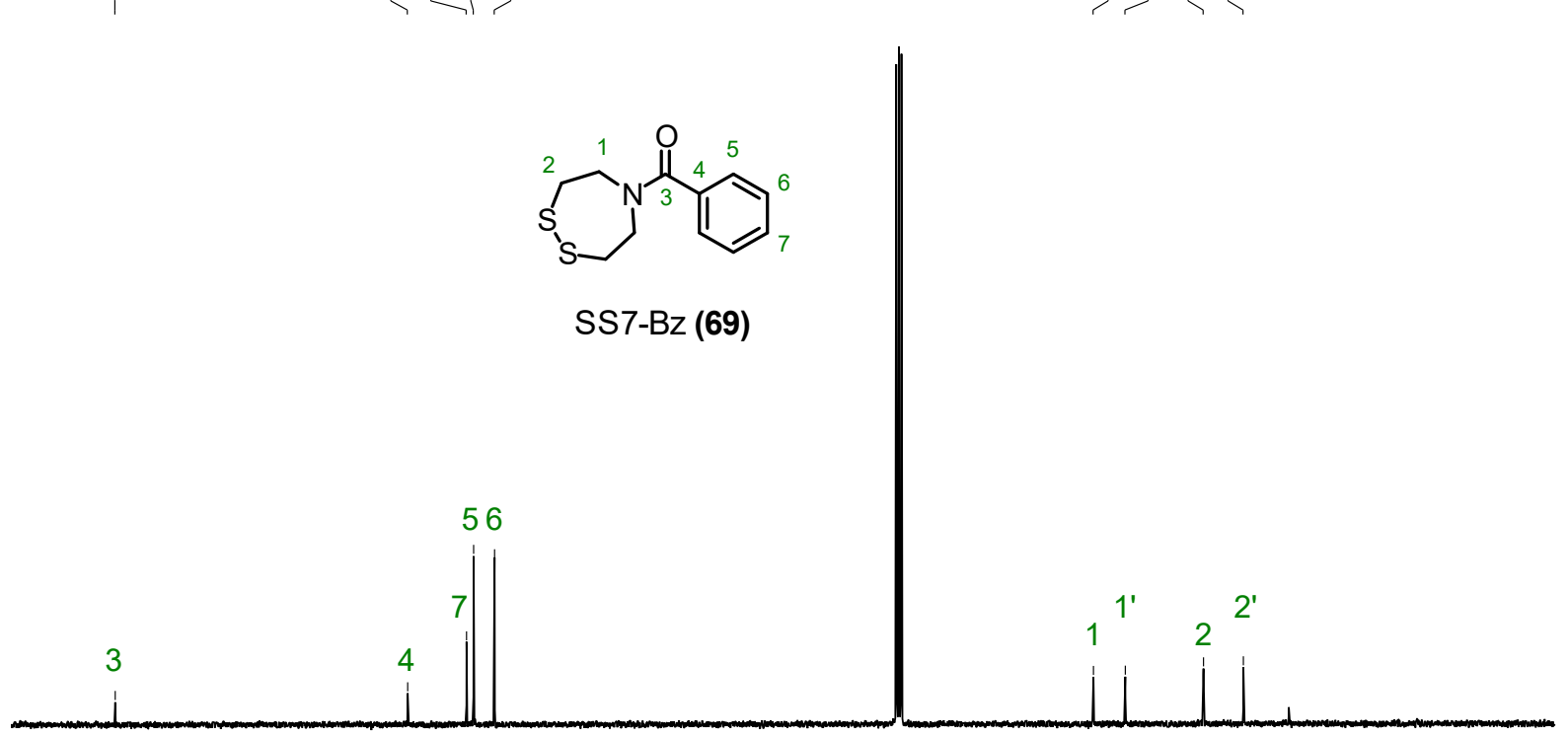

180

160

140

120

100

80

60

40

20

$\delta(\mathrm{ppm})$ 
trans-chloro-2-(chloro-4-oxo-3,4-dihydroquinazolin-2-yl)phenyl trans-hexahydro-[1,2]dithiino $[4,5-b]$ pyridine-1 $(2 H)$-carboxylate (SS66T-PQ, 70)

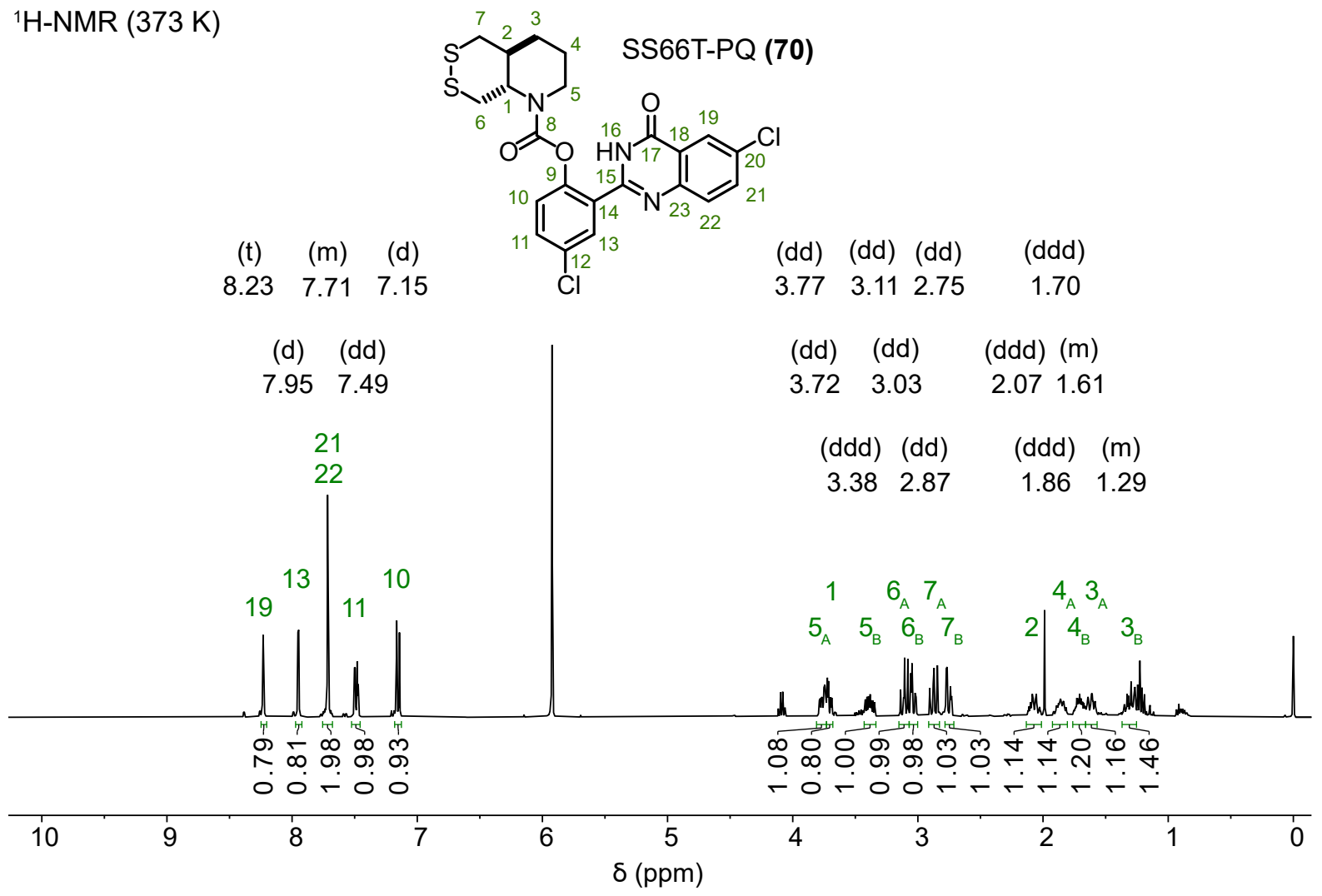

${ }^{13} \mathrm{C}-\mathrm{NMR}(373 \mathrm{~K})$

$\forall$ ำ ○ r T r m N<smiles>O=C(Oc1ccc(Cl)cc1-c1nc2ccc(Cl)cc2c(=O)[nH]1)N1CCC[C@H]2CSSC[C@H]21</smiles>

11

160

140

120

100

$\delta(\mathrm{ppm})$ 
cis-chloro-2-(chloro-4-oxo-3,4-dihydroquinazolin-2-yl)phenyl-hexahydro-[1,2]dithiino [4,5-b]pyridine-1 (2H)-carboxylate (SS66C-PQ, 71)

${ }^{1} \mathrm{H}-\mathrm{NMR}$

(d) (t)

7.997 .50

(s)

(t) $\quad(\mathrm{m})$

(d)

10.15

8.267 .73<smiles>O=C(OCc1ccccc1)Oc1ccc(Cl)cc1-c1nc2ccc(Cl)cc2c(=O)[nH]1</smiles>

(dd)

$\begin{array}{llll}(\mathrm{dd}) & (\mathrm{td}) \quad(\mathrm{dt}) \quad(\mathrm{m})\end{array}$

$\begin{array}{llll}3.47 & 2.79 & 2.20 & 1.62\end{array}$

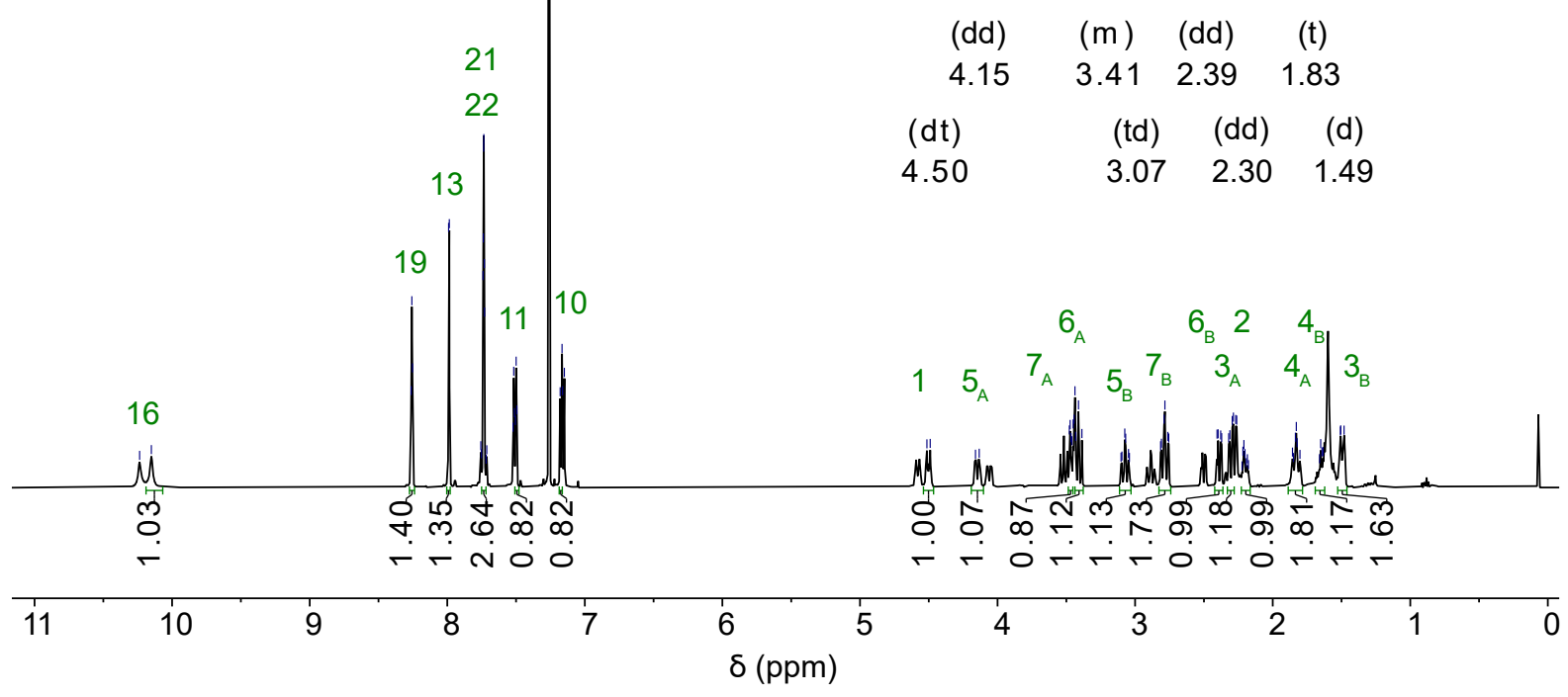

${ }^{13} \mathrm{C}-\mathrm{NMR}$

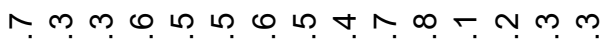
○ी

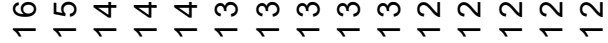

m.

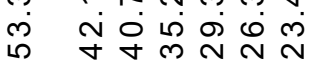

r.j.<smiles></smiles>

1119

13

2221,10

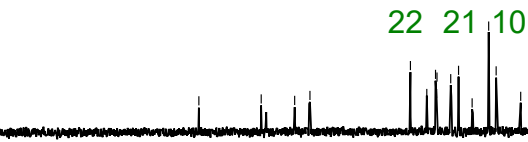

160

140

120

100

$\delta(\mathrm{ppm})$ 
cis-chloro-2-(chloro-4-oxo-3,4-dihydroquinazolin-2-yl)phenyl-hexahydrofuro-[3,4-b]pyridine$1(2 H)$-carboxylate $(\mathrm{O} 56-\mathrm{PQ}, 72)$

${ }^{1} \mathrm{H}-\mathrm{NMR}$

$\begin{array}{ccc}(t) & (m) & (d) \\ 8.23 & 7.71 & 7.17\end{array}$<smiles></smiles>

(s)

(d) (dd)

9.65

$\begin{array}{ll}7.96 & 7.48\end{array}$

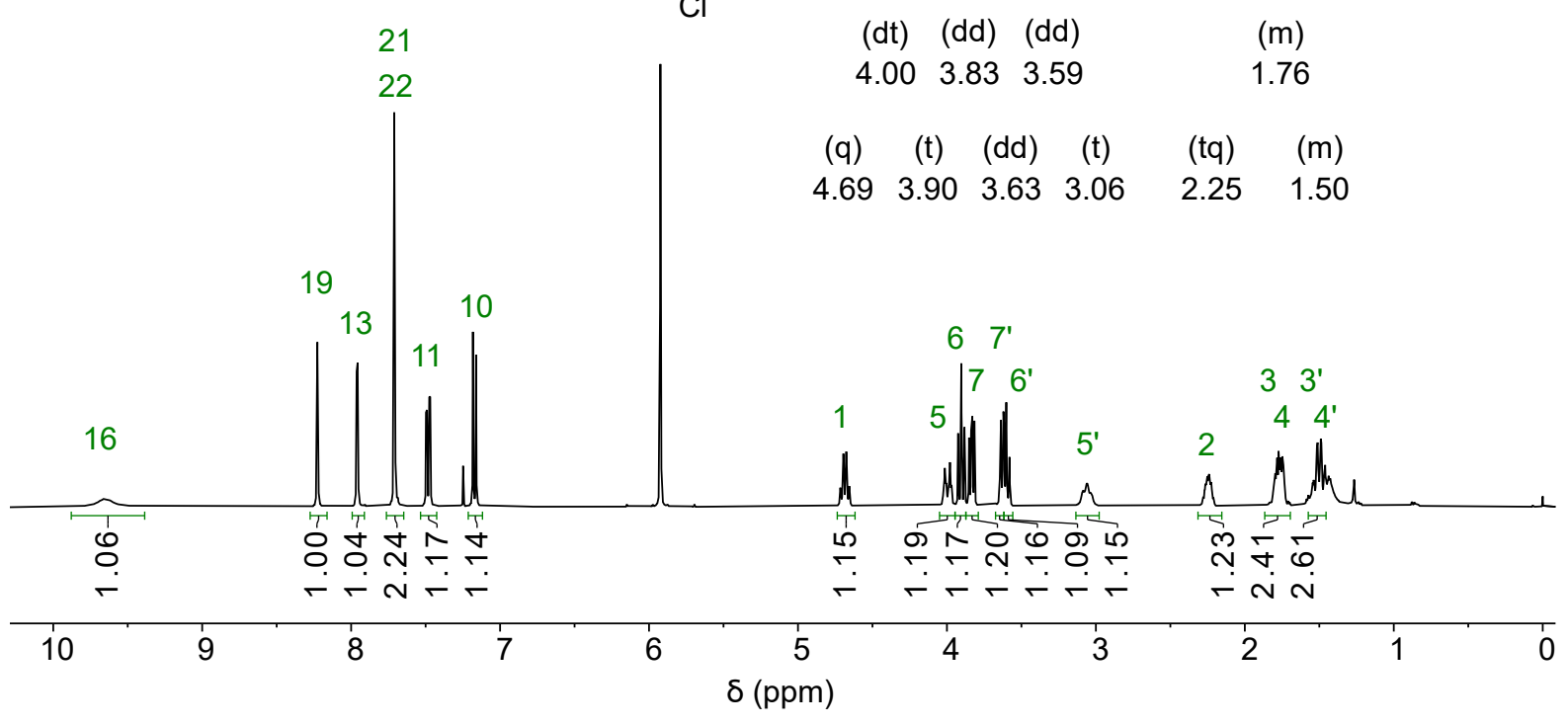

${ }^{13} \mathrm{C}-\mathrm{NMR}$

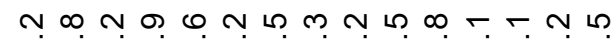

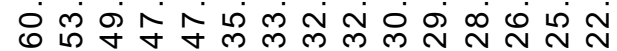

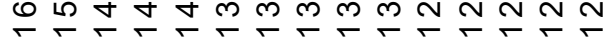

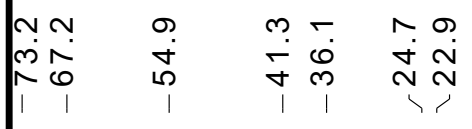<smiles></smiles>

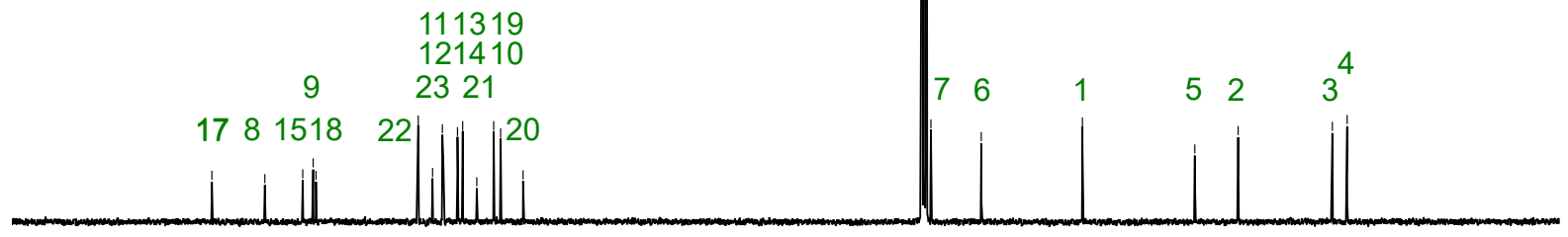

180

160

140

120

100

$\delta(\mathrm{ppm})$

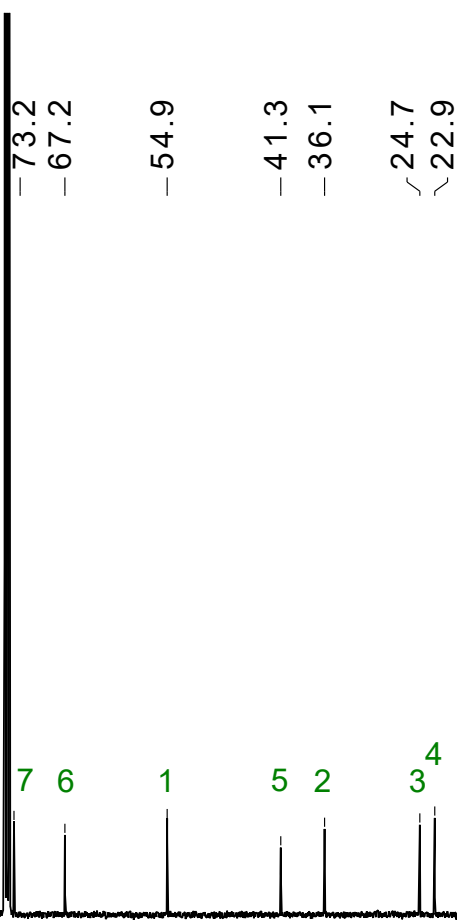


${ }^{1} \mathrm{H}-\mathrm{NMR}(373 \mathrm{~K})$

(t) $\quad(\mathrm{m}) \quad(\mathrm{d})$

8.227 .717 .17

(s) (d) (dd)

9.56

7.977 .49

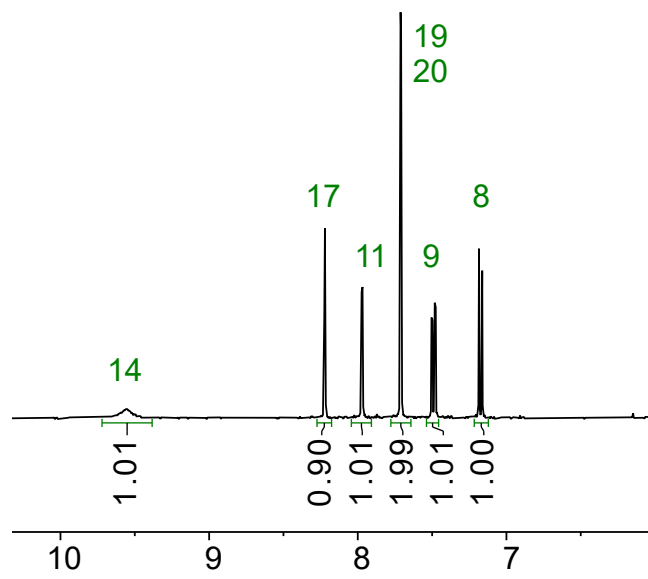

${ }^{13} \mathrm{C}-\mathrm{NMR}(373 \mathrm{~K})$

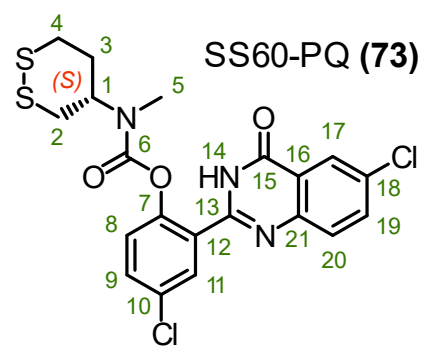

$\begin{array}{cc}(\mathrm{ddd}) & (\mathrm{s}) \\ 3.15 & 2.97\end{array}$

(dd) (dd)

$3.10 \quad 2.72$

(d) $\quad(\mathrm{t}) \quad(\mathrm{m})$

$\begin{array}{lll}4.17 & 2.93 \quad 2.05\end{array}$

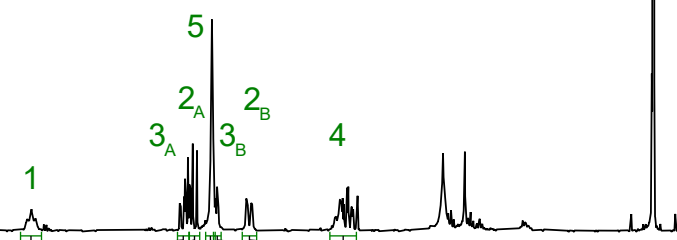

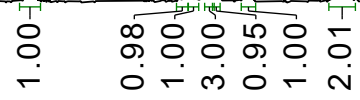

o. ○ं भं⿰彳

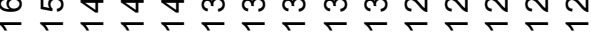<smiles>CN(C(=O)Oc1ccc(Cl)cc1-c1nc2ccc(Cl)cc2c(=O)[nH]1)C1CCSCC1OS(=O)(=O)O</smiles>

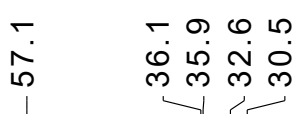

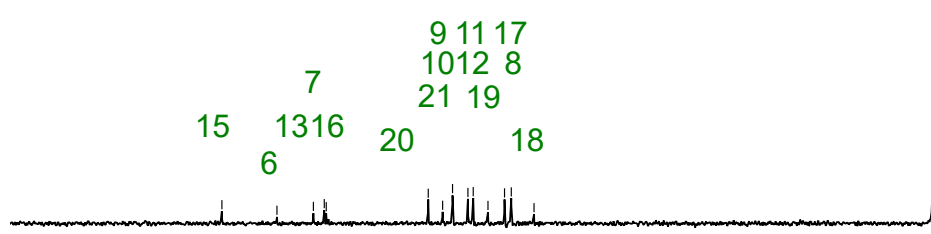

$$
1 \quad 35
$$

\begin{tabular}{|c|c|c|c|c|c|c|c|}
\hline 180 & 160 & 140 & 120 & 100 & 80 & 60 & 40 \\
\hline
\end{tabular}


chloro-2-(chloro-4-oxo-3,4-dihydroquinazolin-2-yl)phenyl 1,2,5-dithiazepane-carboxylate (SS7-PQ, 75)

${ }^{1} \mathrm{H}-\mathrm{NMR}$

(d) $\quad(\mathrm{dd})$

7.907 .53

$\begin{array}{llll}(\mathrm{s}) & (\mathrm{dd}) \quad(\mathrm{m}) \quad(\mathrm{d})\end{array}$

$\begin{array}{llll}10.04 & 8.28 & 7.73 & 7.19\end{array}$
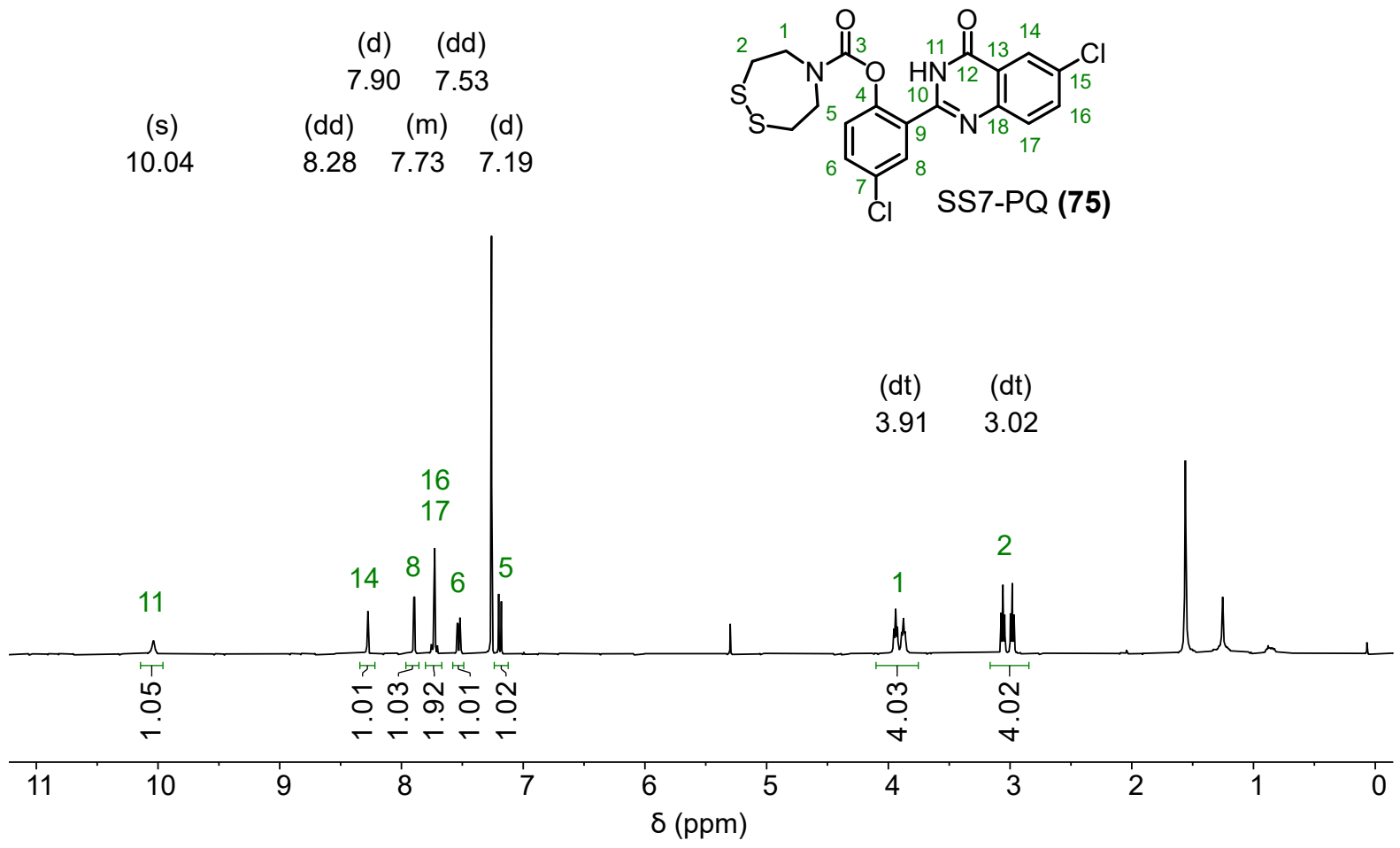

${ }^{13} \mathrm{C}-\mathrm{NMR}$

$0 . t \nabla m$ t

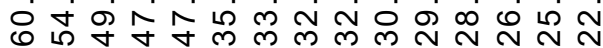

i) $\infty$ \%

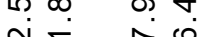

in in ले<smiles>O=C(Oc1ccc(Cl)cc1-c1nc2ccc(Cl)cc2c(=O)[nH]1)N1CCSSCC1</smiles>

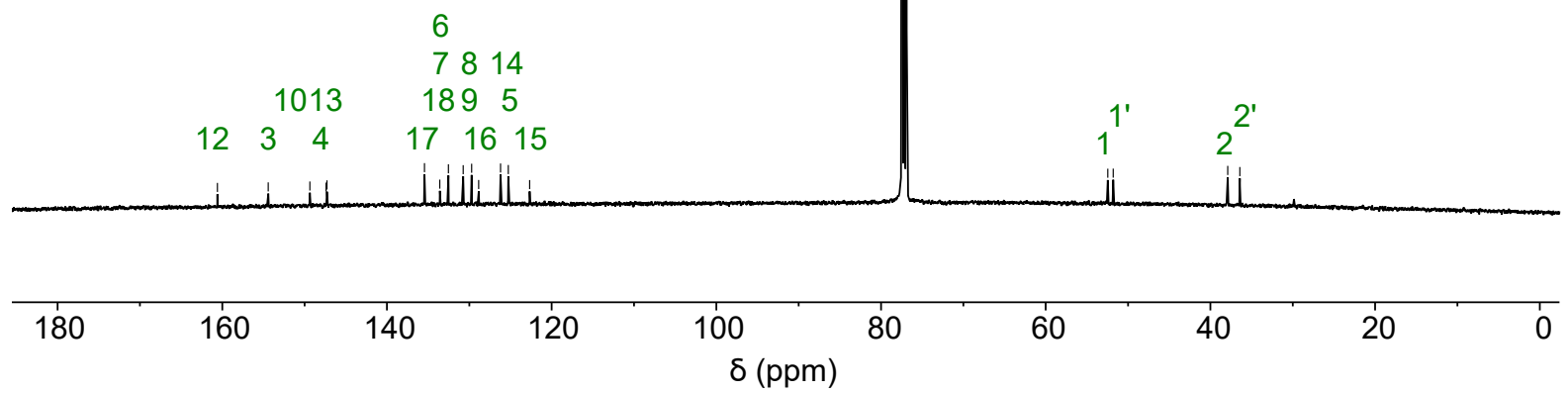


chloro-2-(chloro-4-oxo-3,4-dihydroquinazolin-2-yl)phenyl methyl(2-((2-(methylamino)ethyl) disulfaneyl)ethyl)carbamate (SSO0-PQ, 76)

${ }^{1} \mathrm{H}-\mathrm{NMR}\left(\right.$ tet-Cl $\left.{ }_{4}, 373 \mathrm{~K}\right)$

(t) $\quad(d) \quad(d)$

$8.20 \quad 7.71 \quad 7.24$

(d) (dd)

7.877 .48

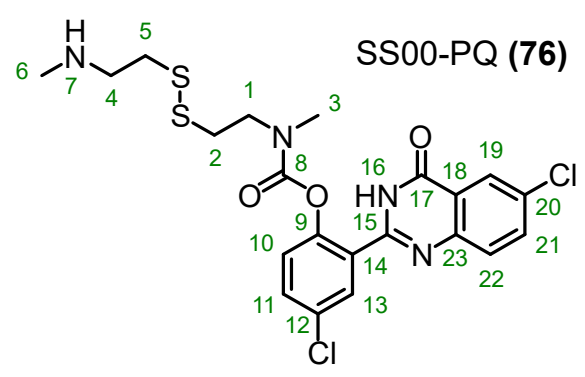

(d) (s) (s)

$3.23 \quad 3.03 \quad 2.66$

(s) (d) (s)

$\begin{array}{lll}3.62 & 3.18 & 2.88\end{array}$

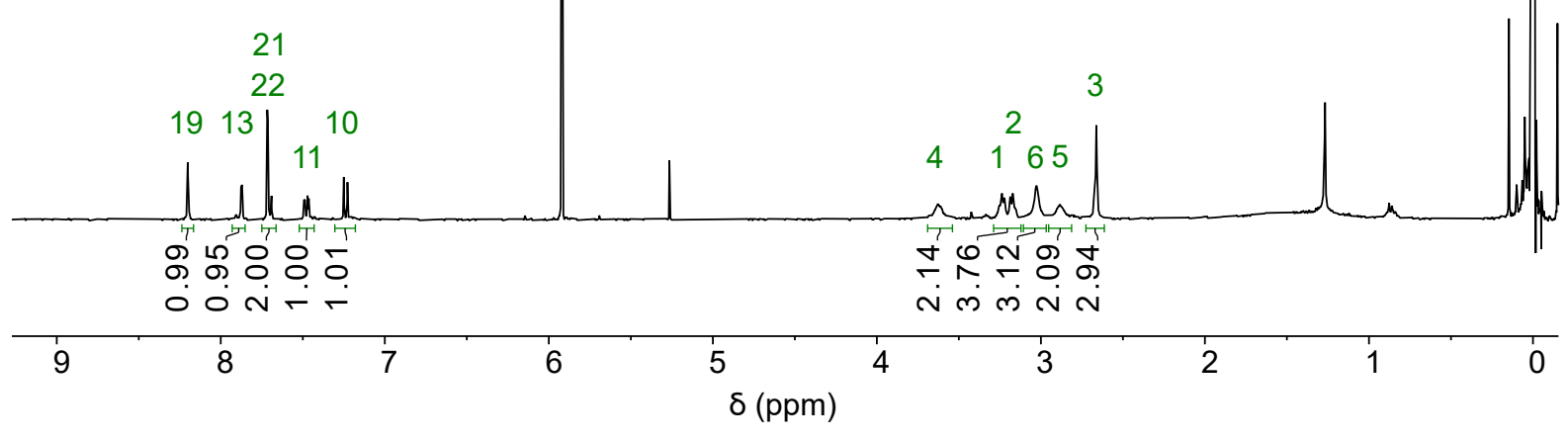

${ }^{13} \mathrm{C}-N M R\left(\right.$ DMSO- $\left.d_{6}, 373 \mathrm{~K}\right)$

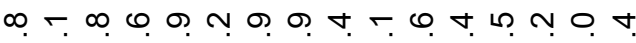

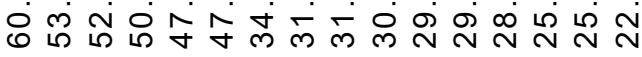

$r-r-r T r \frac{T}{2}$<smiles>CNCCSSCCN(C)C(=O)Oc1ccc(Cl)cc1-c1nc2ccc(Cl)cc2c(=O)[nH]1</smiles>
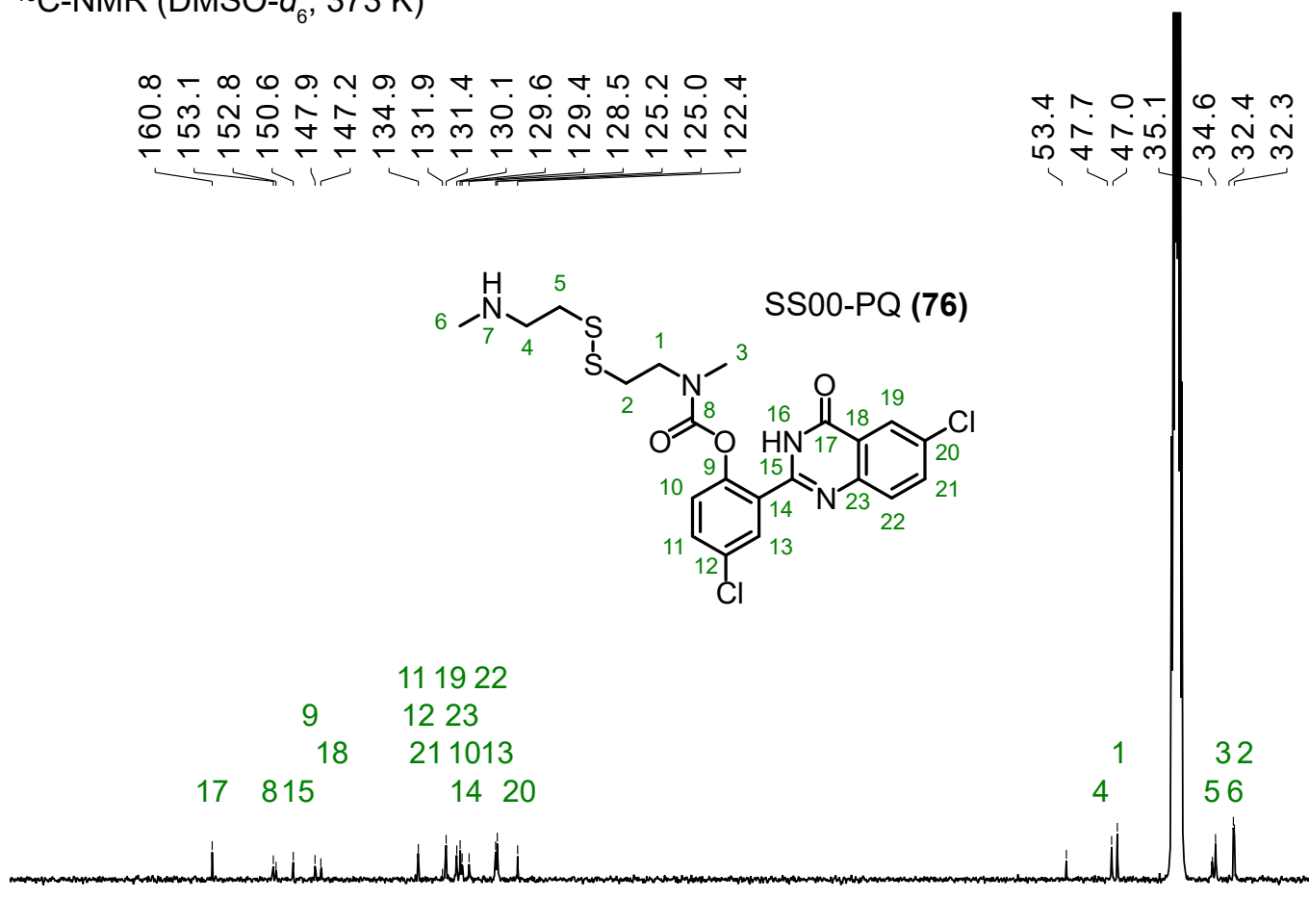

180

160

140

120

100

80

60

40

20

$\delta(\mathrm{ppm})$ 
chloro-2-(chloro-4-oxo-3,4-dihydroquinazolin-2-yl)phenyl 2-(((piperidin-2-ylmethyl)disulfaneyl) methyl)piperidine-carboxylate (SS06-PQ, 77)

${ }^{1} \mathrm{H}-\mathrm{NMR}(373 \mathrm{~K})$

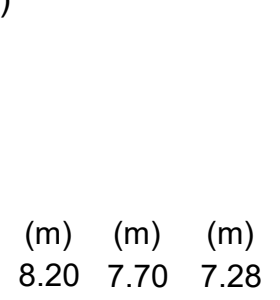

(t) $\quad(\mathrm{m})$

$7.91 \quad 7.47$

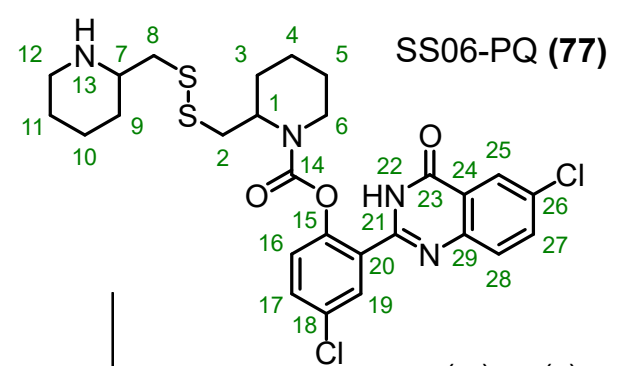

$\begin{array}{llll}(\mathrm{m}) & (\mathrm{s}) \quad(\mathrm{m}) \quad(\mathrm{t})\end{array}$

$\begin{array}{llll}3.53 & 3.11 & 1.92 & 1.65\end{array}$

$\begin{array}{lllll}(d) & (m) & (d d) & (m) & (d d)\end{array}$

$\begin{array}{lllll}4.05 & 3.33 & 3.01 & 1.86 & 1.46\end{array}$

(s) $\quad(\mathrm{dd}) \quad(\mathrm{m})$

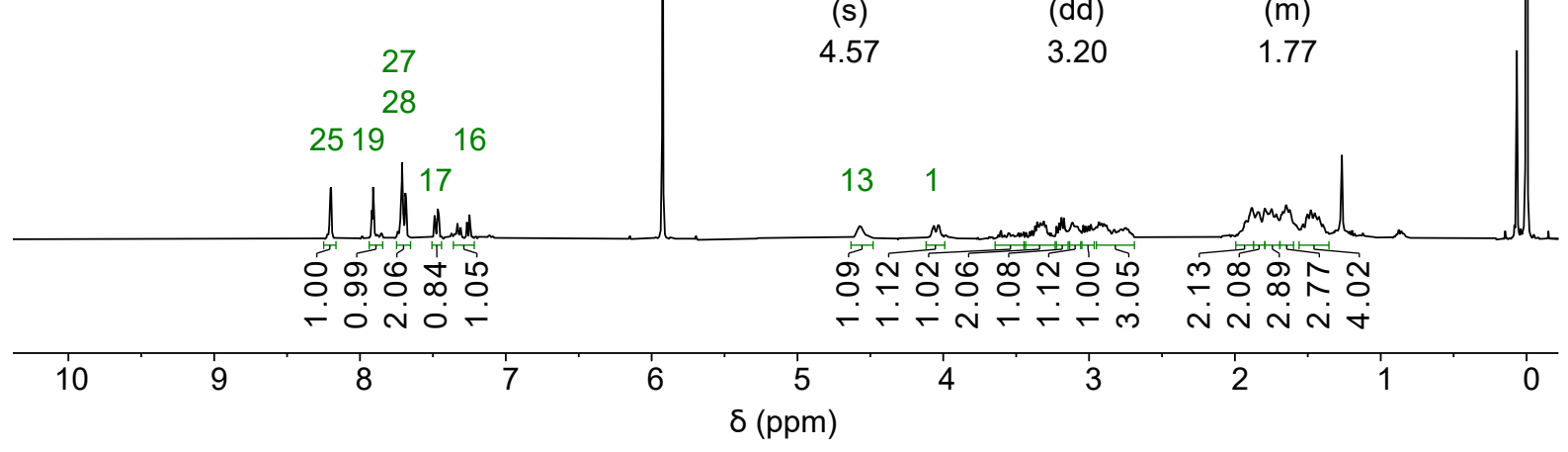

${ }^{13} \mathrm{C}-\mathrm{NMR}(373 \mathrm{~K})$

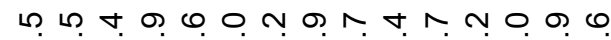

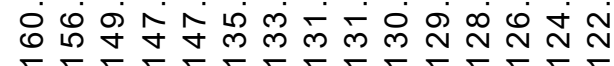

mim-ntamo not 它 ம

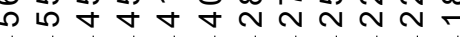<smiles>O=C(Oc1ccc(Cl)cc1)c1nc2ccc(Cl)cc2c(=O)[nH]1</smiles>

281825

$$
\begin{array}{ccc} 
& 24 & 2919 \\
14 & 15 & 172716 \\
23 & 2128 & 20 \quad 26
\end{array}
$$

1

7

\begin{tabular}{cccccccccc}
\hline 180 & 160 & 140 & 120 & 100 & 1 & 1 \\
$\delta(\mathrm{ppm})$ & 80 & 60 & 40 & 20 & 0
\end{tabular}


trans-3'-methoxy-3-oxo-3H-spiro[isobenzofuran-1,9'-xanthen]-6'-yl hexahydro-[1,2]dithiino [4,5-b]pyridine-1 $(2 H)$-carboxylate (SS66T-MF, 78)

${ }^{1} \mathrm{H}-\mathrm{NMR}$

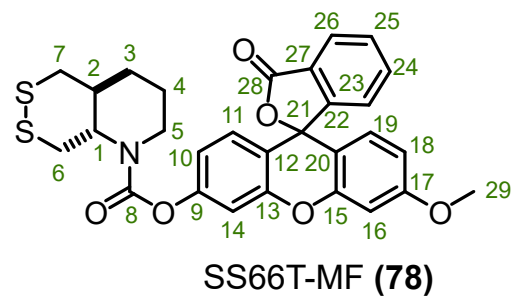

$(\mathrm{t}) \quad(\mathrm{d}) \quad(\mathrm{d})$

$\begin{array}{lll}7.62 & 7.15 & 6.70\end{array}$

(tt) $\quad(\mathrm{dd}) \quad(\mathrm{d})$

$7.67 \quad 7.096 .76$

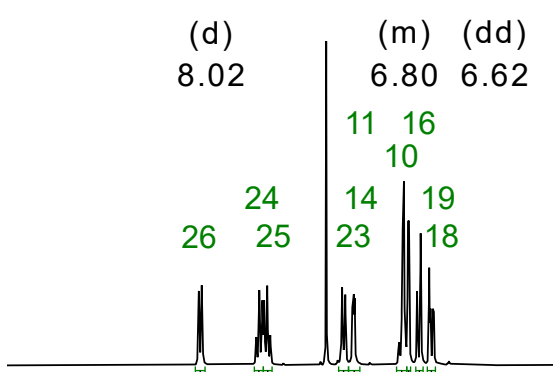

80,0000000

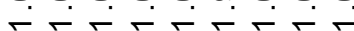

(s)

3.84

(d)

$\begin{array}{llll}(m) & \text { (d) }(d d) & (m)\end{array}$

$3.90 \quad 3.352 .81 \quad 1.78$

29

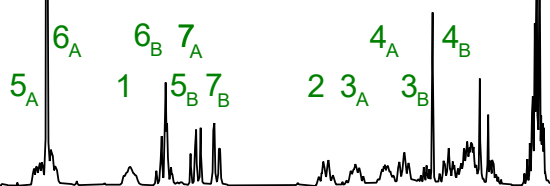

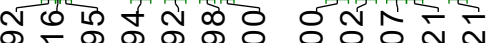

omórirrir

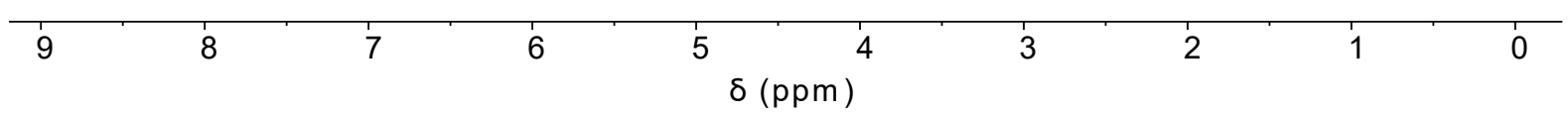

${ }^{13} \mathrm{C}-\mathrm{NMR}$

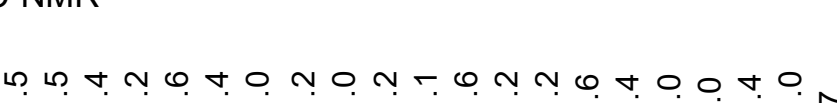

बํ்

- N T TOMn

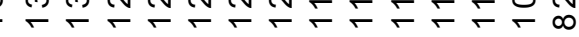

峞

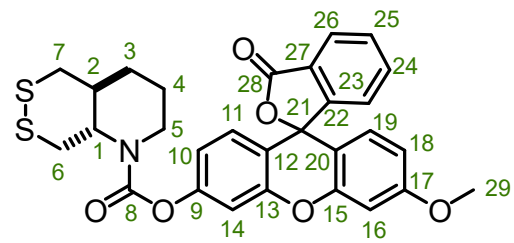

SS66T-MF (78)

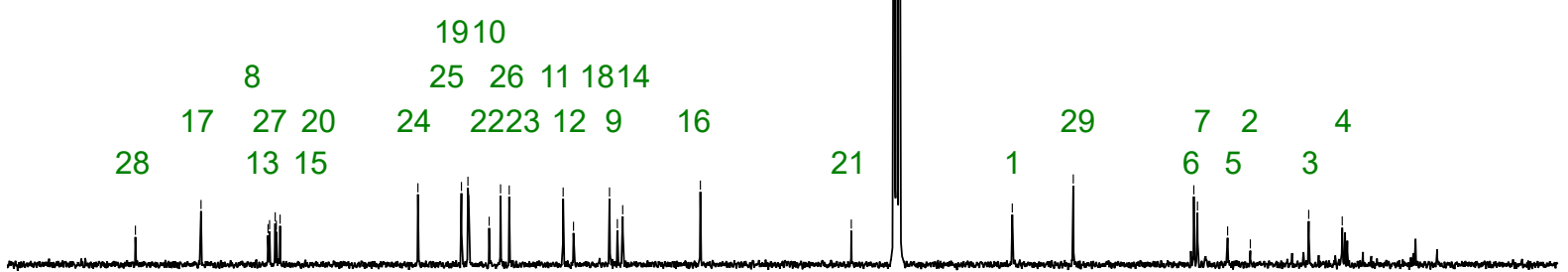

180

160

140

120

100

80

60

40

20

$\delta(p p m)$ 
cis-3'-methoxy-3-oxo-3H-spiro[isobenzofuran-1,9'-xanthen]-6'-yl hexahydro-[1,2]dithiino [4,5-b]pyridine-1 $(2 H)$-carboxylate (SS66C-MF, 79)

${ }^{1} \mathrm{H}-\mathrm{NMR}$

SS66C-MF (79)

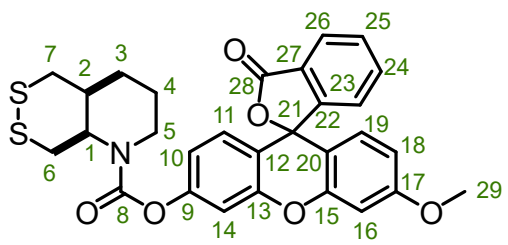

(d) $\quad(\mathrm{m}) \quad(\mathrm{d}) \quad(\mathrm{dd})$

$\begin{array}{llll}8.02 & 7.07 & 6.76 & 6.62\end{array}$

(t) $\quad(\mathrm{m})$

7.67

6.79

(t) (d) (d)

7.627 .166 .70

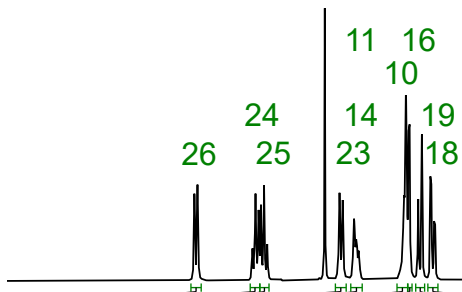

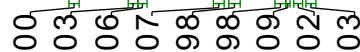

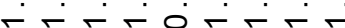

4.14

$(\mathrm{t})$

4.56

29 (s) (ddt) (m)

$3.84 \quad 2.51 \quad 1.66$

$(\mathrm{t}) \quad(\mathrm{d}) \quad(\mathrm{m})$

$2.83 \quad 1.86 \quad 1.28$

(m) (dt) (m) (d)

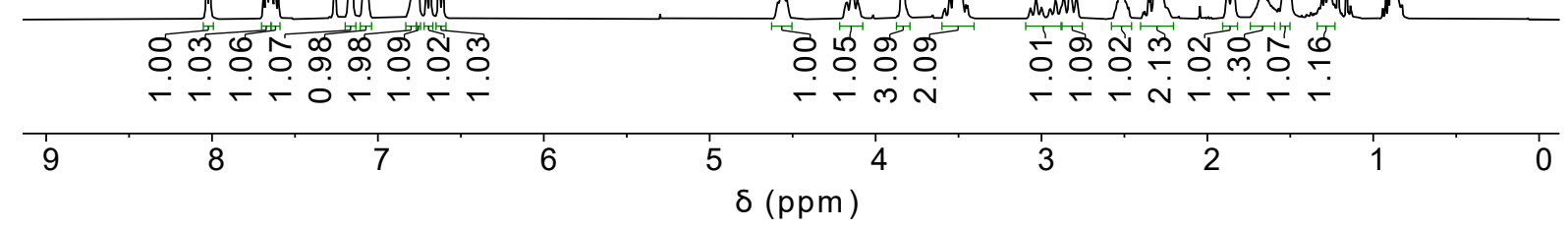

${ }^{13} \mathrm{C}-\mathrm{NMR}$

n

๑ே
N $-m m$ m n N R t ம்

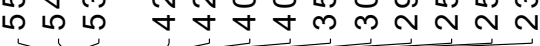

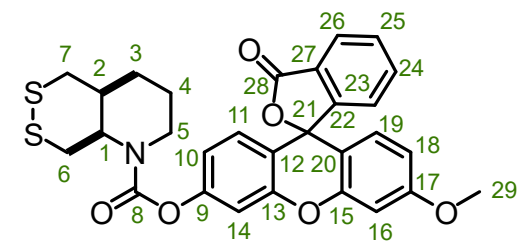

SS66C-MF (79)

\begin{tabular}{|c|c|c|c|c|}
\hline \multicolumn{4}{|c|}{1910} & \\
\hline & 8 & & $5 \quad 26 \quad 111814$ & \\
\hline 17 & 2720 & 24 & 2223129 & \\
\hline & 1315 & & & \\
\hline
\end{tabular}

180

160

140

120

100

$\delta(\mathrm{ppm})$

$\begin{array}{llll}29 & 7 & 2 & 4\end{array}$

1653 
cis-3'-methoxy-3-oxo-3H-spiro[isobenzofuran-1,9'-xanthen]-6'-yl hexahydrofuro[3,4-b]pyridine-1(2H)-carboxylate (O56-MF, 80)

${ }^{1} \mathrm{H}-\mathrm{NMR}$
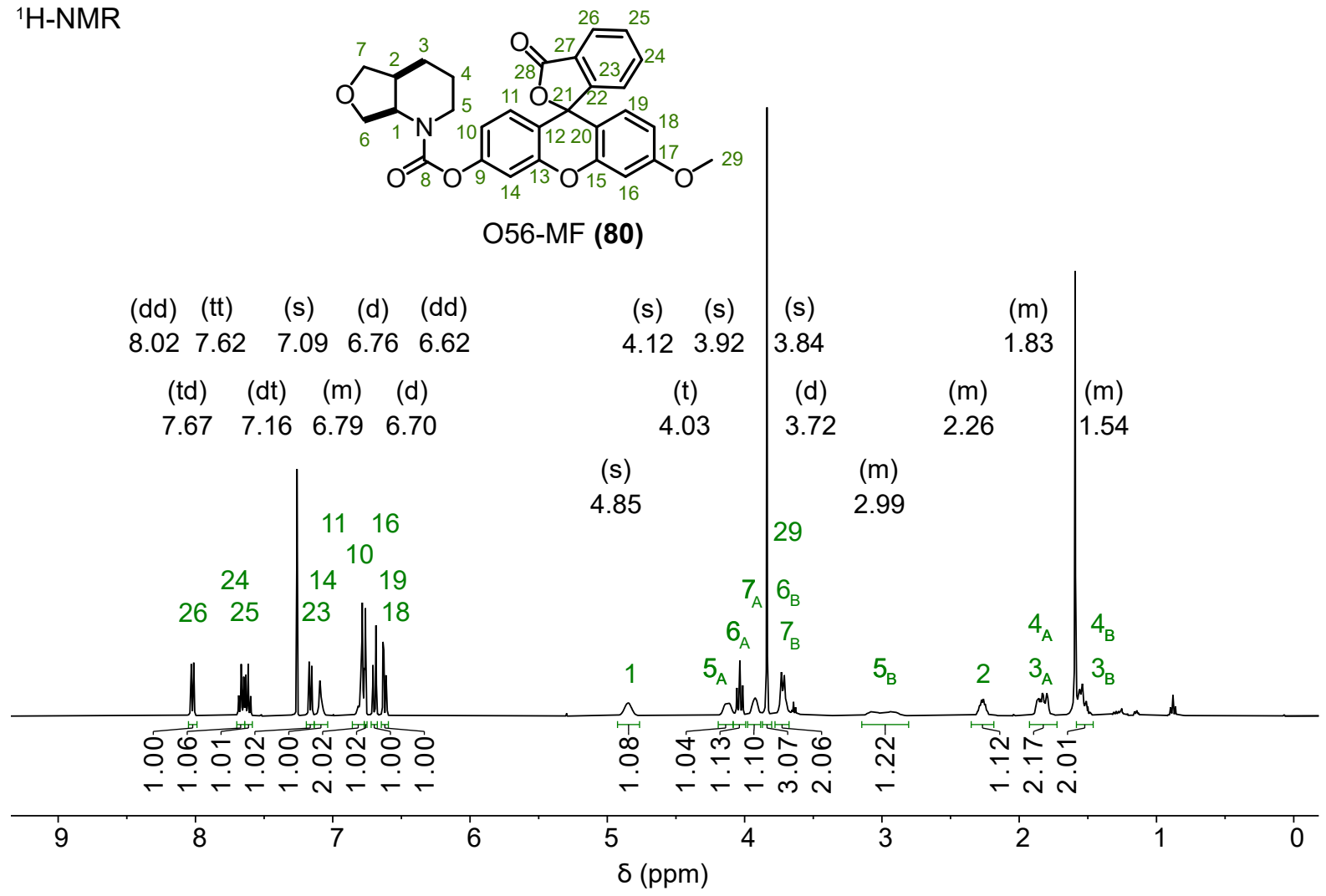

${ }^{13} \mathrm{C}-\mathrm{NMR}$

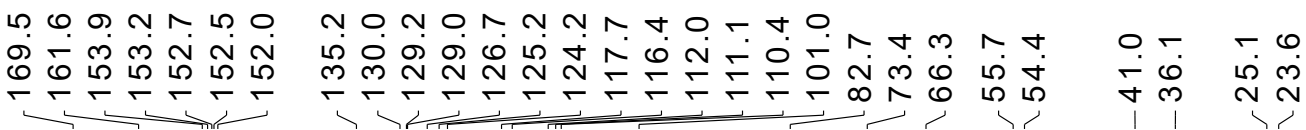
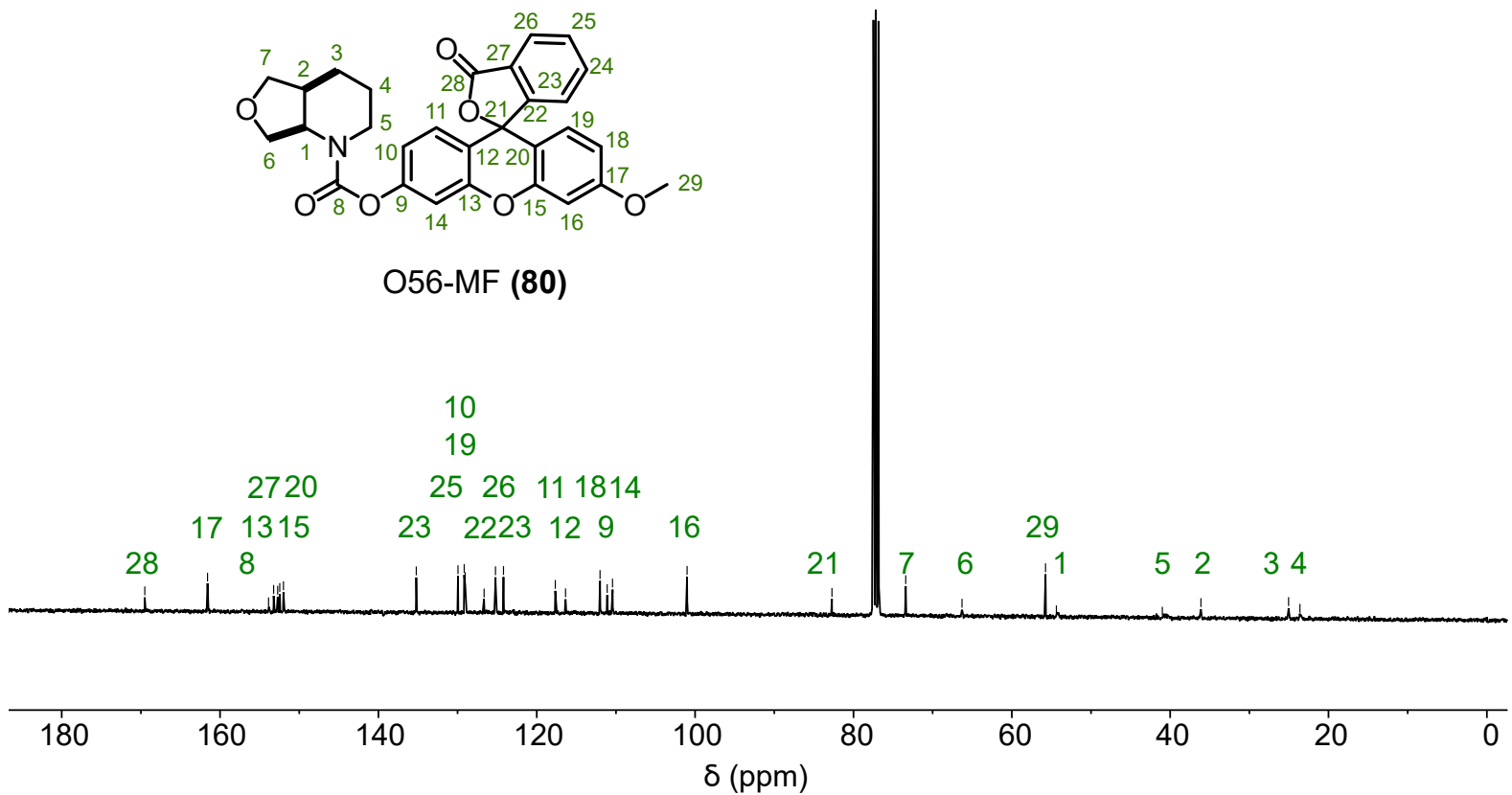
3'-methoxy-3-oxo-3H-spiro[isobenzofuran-1,9'-xanthen]-6'-yl ((S)-1,2-dithian-4yl)(methyl)carbamate (SS60-MF, 81)

${ }^{1} \mathrm{H}-\mathrm{NMR}$

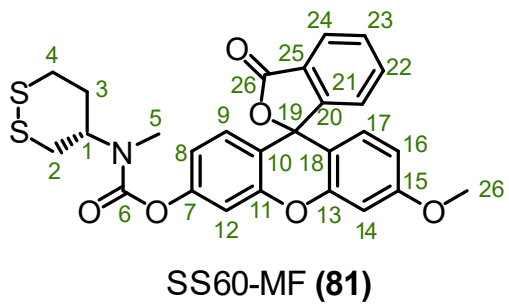

$$
\text { (dd) }(\text { td }) \quad(d) \quad(d) \quad(d d)
$$

$8.027 .62 \quad 7.096 .76 \quad 6.62$

(s)

$(\mathrm{m})$

3.01

$\begin{array}{llll}(\mathrm{td}) & (\mathrm{dt}) & (\mathrm{m}) & (\mathrm{d}) \\ 7.67 & 7.16 & 6.79 & 6.70\end{array}$

3.84

(t) $\quad(t) \quad(d d)$

(t)

$3.14 \quad 2.80 \quad 2.09$
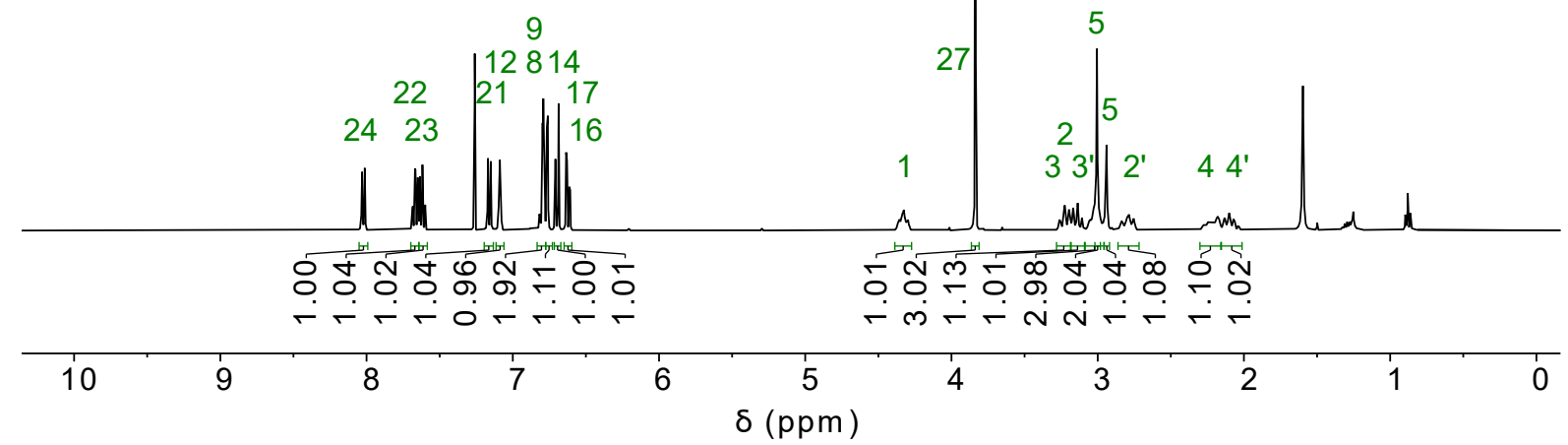

${ }^{13} \mathrm{C}-\mathrm{NMR}$

ம

๑ே

$-\Lambda m \sim-\Lambda \sim 20$

ט

L $m m m m m m$
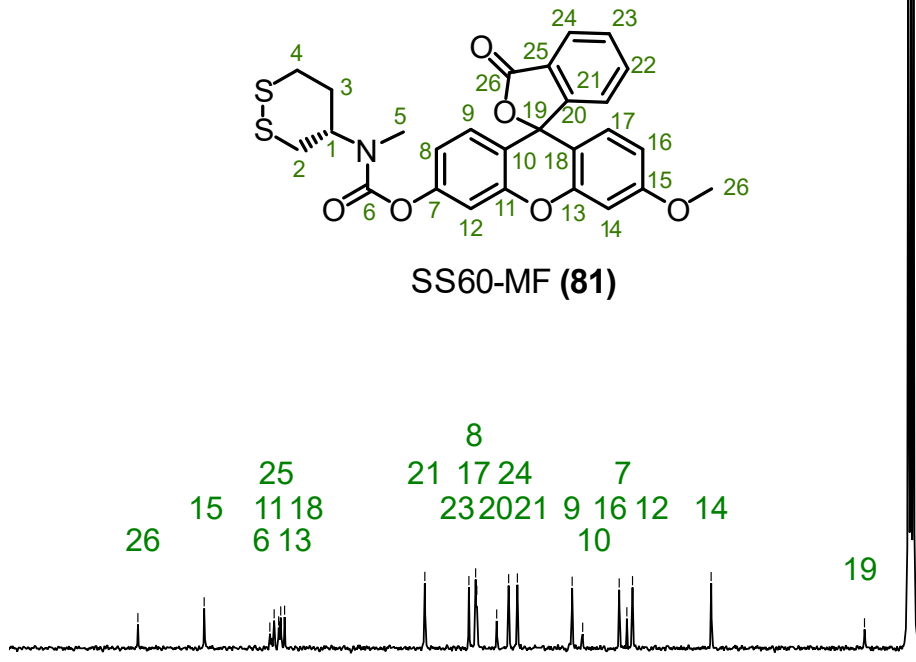

26

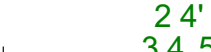

345

180

160

140

120

100

$\delta(\mathrm{ppm})$

Hill 
3'-methoxy-3-oxo-3H-spiro[isobenzofuran-1,9'-xanthen]-6'-yl (1,2-dithiolan-4-yl) (methyl)carbamate (SS50-MF, 82)

${ }^{1} \mathrm{H}-\mathrm{NMR}$

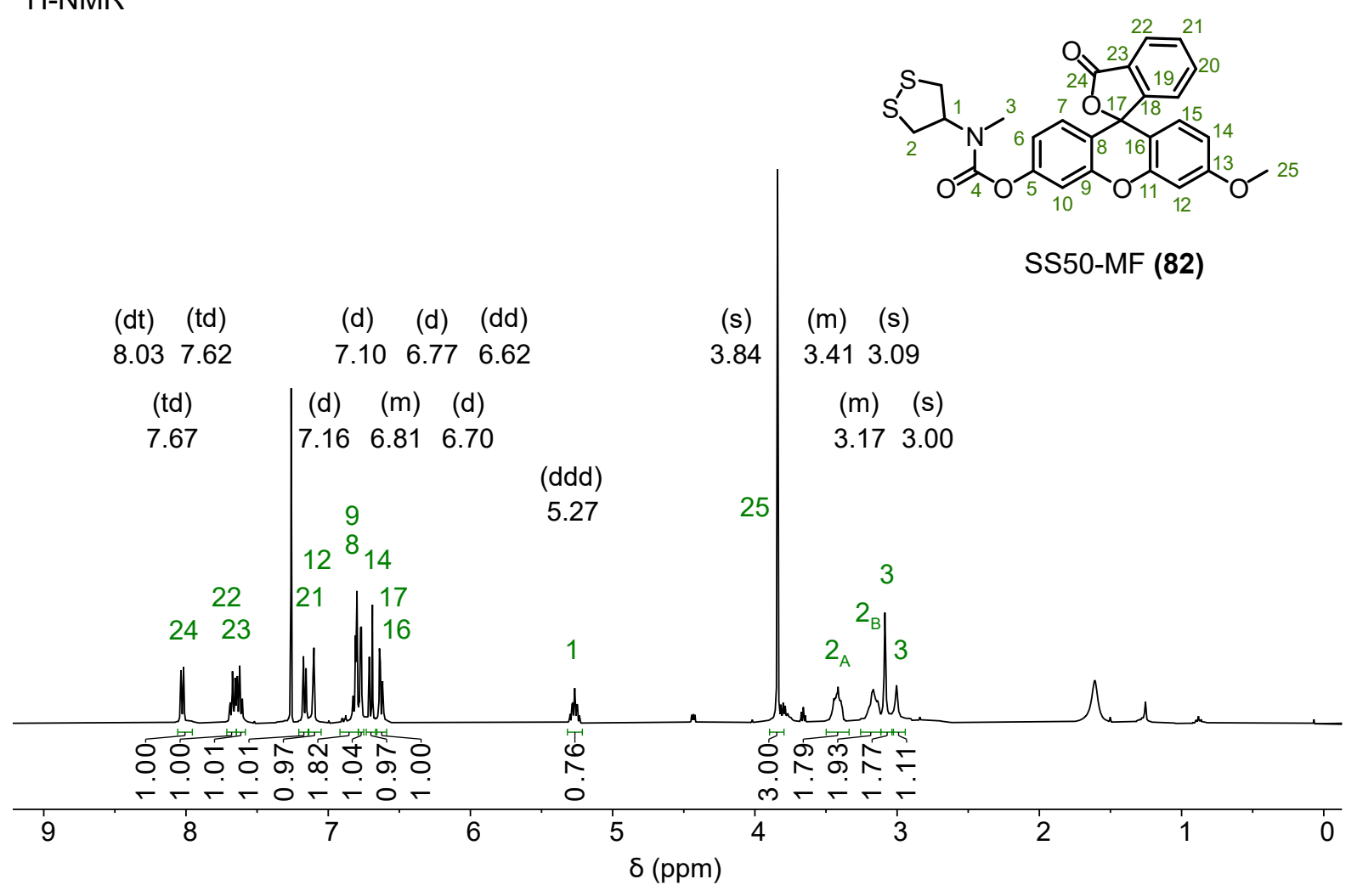

${ }^{13} \mathrm{C}-\mathrm{NMR}$

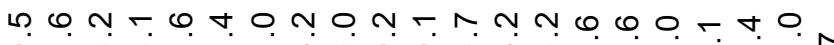

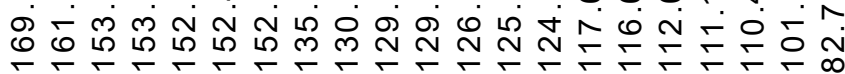

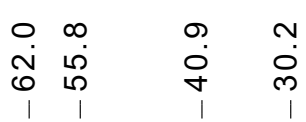

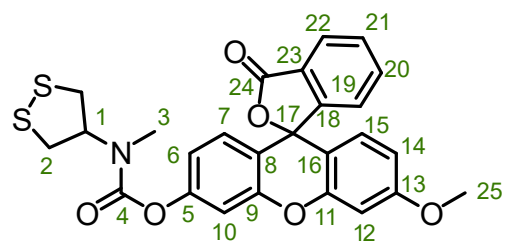

SS50-MF (82)

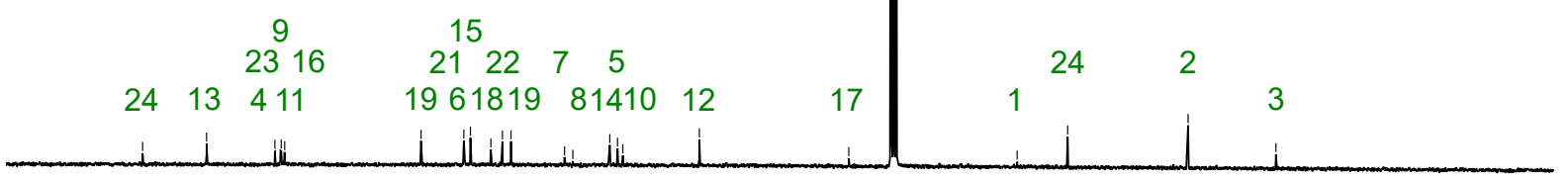

$\begin{array}{cccccccccc}180 & 160 & 140 & 120 & 100 & 80 & 60 & 40 & 20 & 0 \\ \delta(\mathrm{ppm}) & & & & 1\end{array}$


3'-methoxy-3-oxo-3H-spiro[isobenzofuran-1,9'-xanthen]-6'-yl 1,2,5-dithiazepane-carboxylate (SS7-MF, 83)

${ }^{1} \mathrm{H}-\mathrm{NMR}$

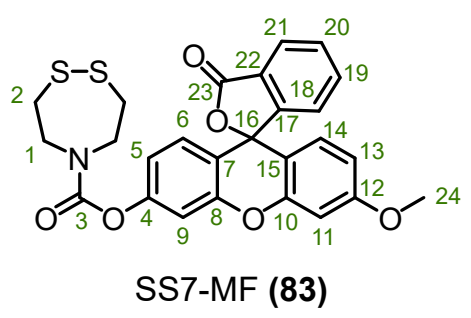
(dd) (td)
(d) $\quad$ (d) (d)
(t) $\quad(\mathrm{s})$

$8.02 \quad 7.62 \quad 7.116 .796 .70$

3.883 .84

$\begin{array}{lllll}(\mathrm{td}) & (\mathrm{dt}) \quad(\mathrm{dd}) \quad(\mathrm{d}) \quad(\mathrm{dd})\end{array}$

$\begin{array}{lllll}7.66 & 7.16 & 6.82 & 6.77 & 6.62\end{array}$

\begin{tabular}{l|l}
$(\mathrm{t})$ & $(\mathrm{t})$
\end{tabular}

$3.96 \quad 3.08$

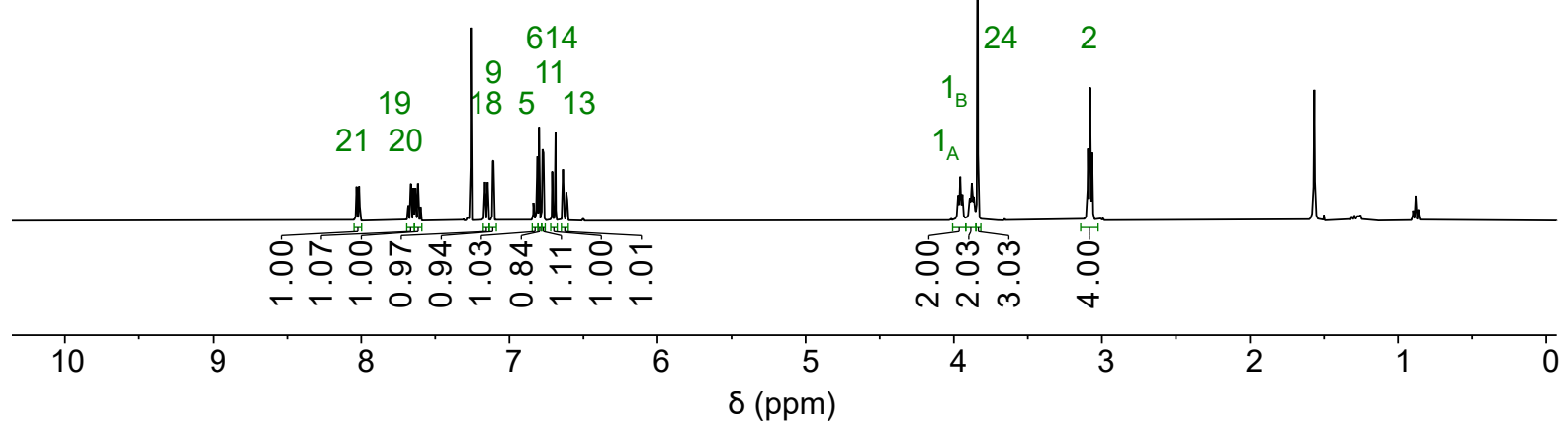

${ }^{13} \mathrm{C}-\mathrm{NMR}$

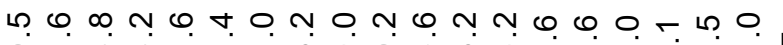

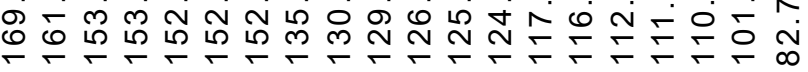

$A-0$ mo

ம0

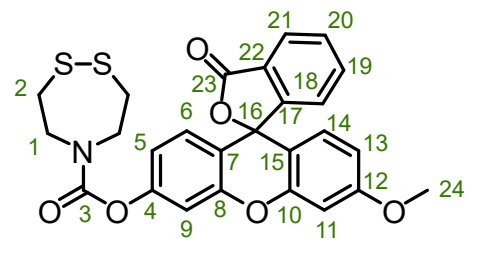

SS7-MF (83)

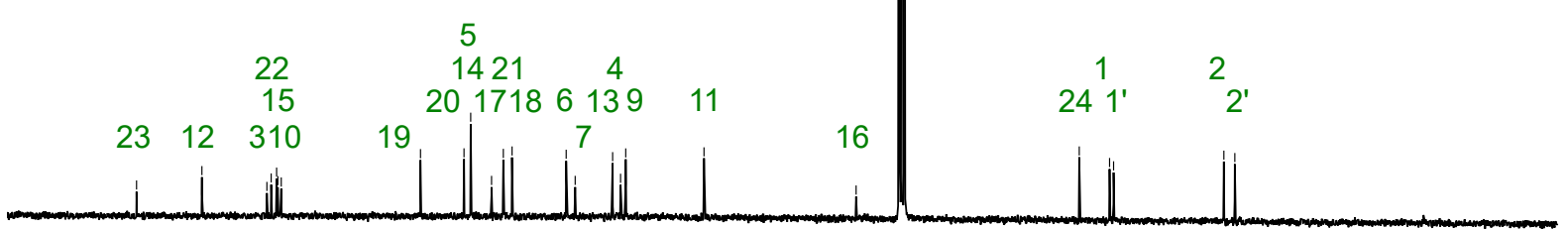

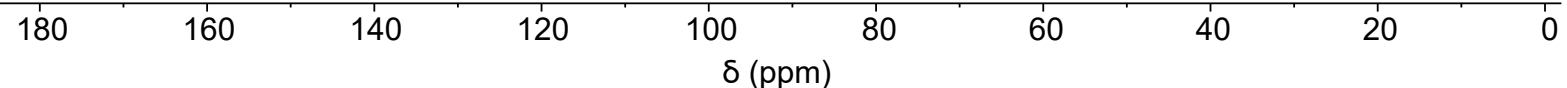


${ }^{1} \mathrm{H}-\mathrm{NMR}$

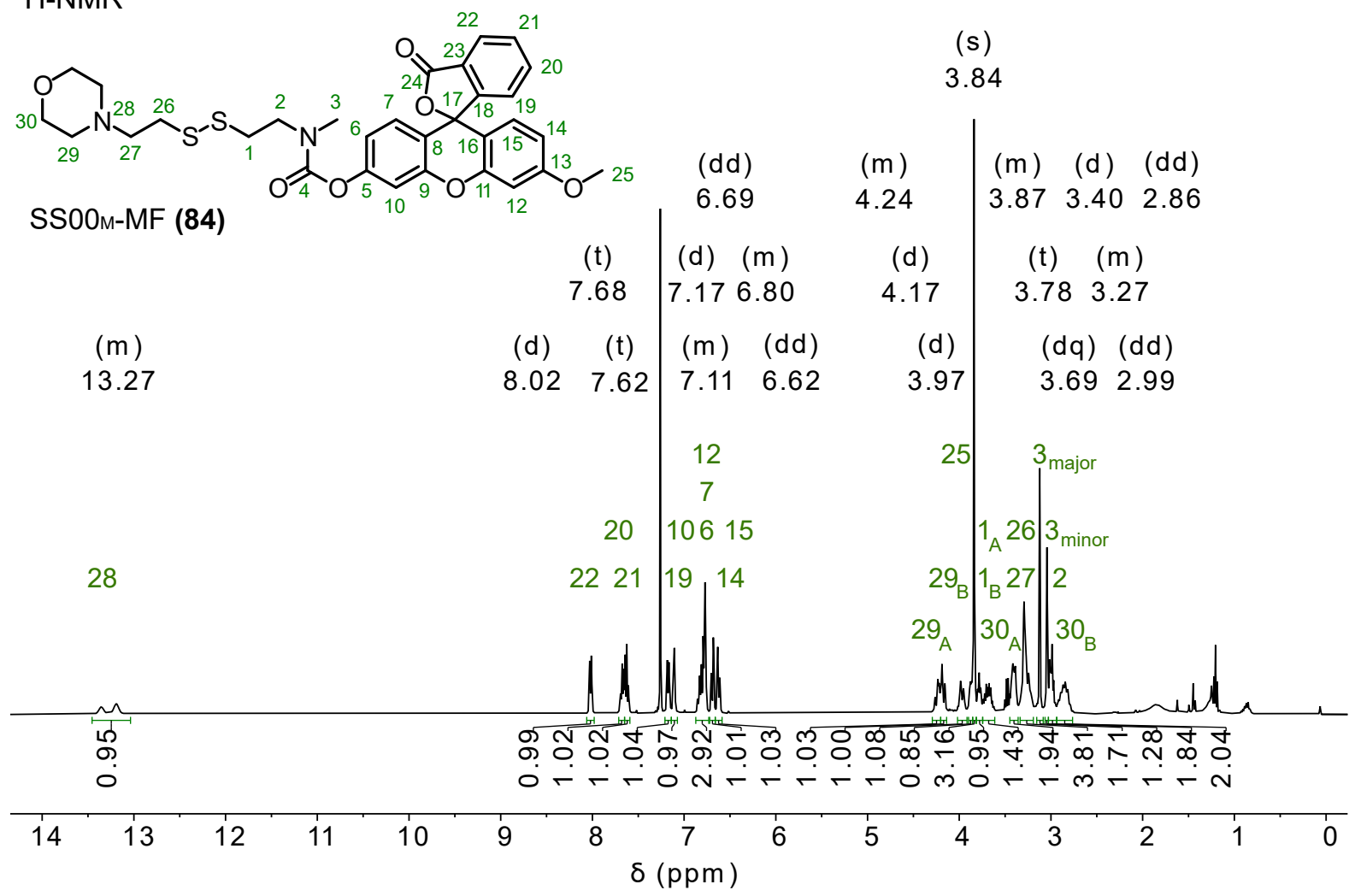

${ }^{13} \mathrm{C}-\mathrm{NMR}$

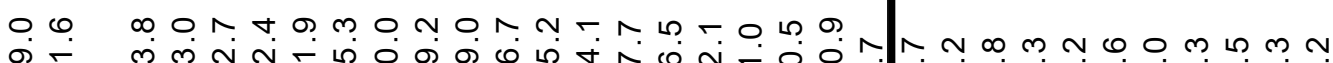

Ð
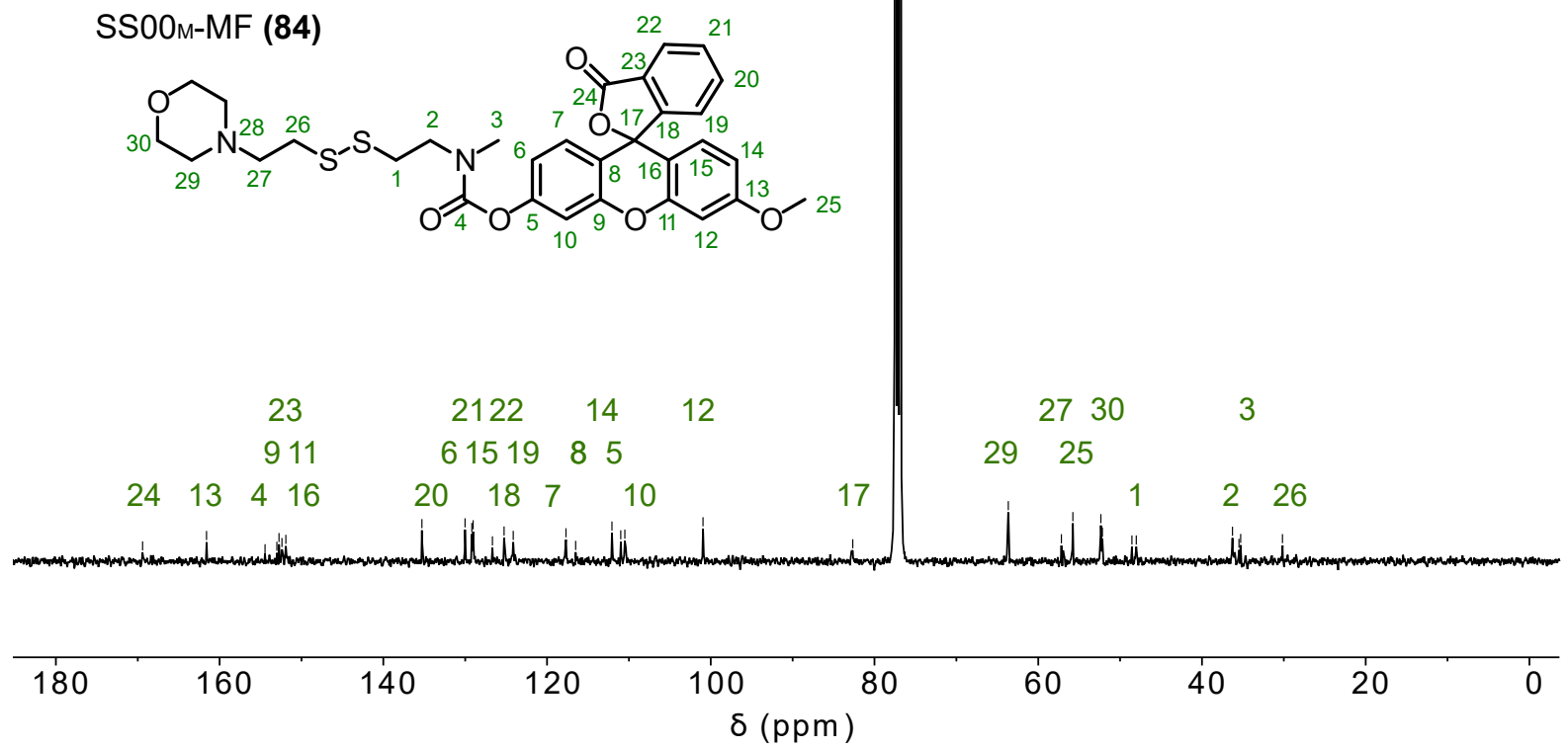
bis(3'-methoxy-3-oxo-3H-spiro[isobenzofuran-1,9'-xanthen]-6'-yl) (disulfanediylbis(ethane-2,1diyl))bis(methylcarbamate) $\left(\mathrm{SSOO}_{-} \mathrm{MF}_{2}, 85\right)$

${ }^{1} \mathrm{H}-\mathrm{NMR}$
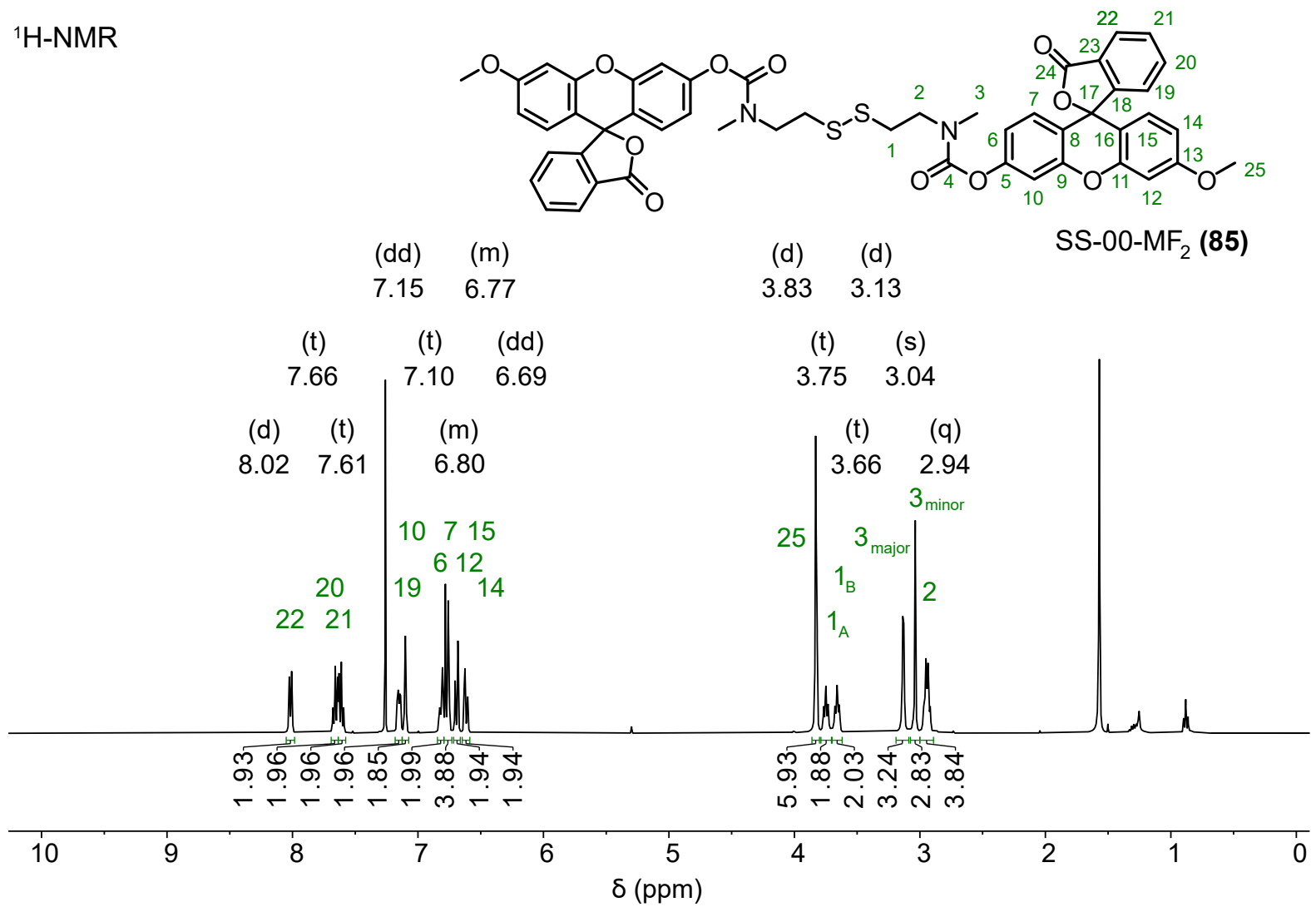

${ }^{13} \mathrm{C}-\mathrm{NMR}$

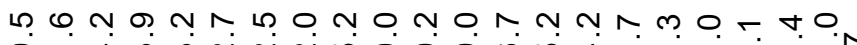

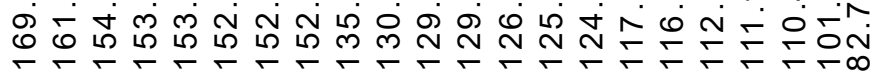

$\wedge \sim 0+\infty 0$

ம

ก $\forall$ m $m$ m
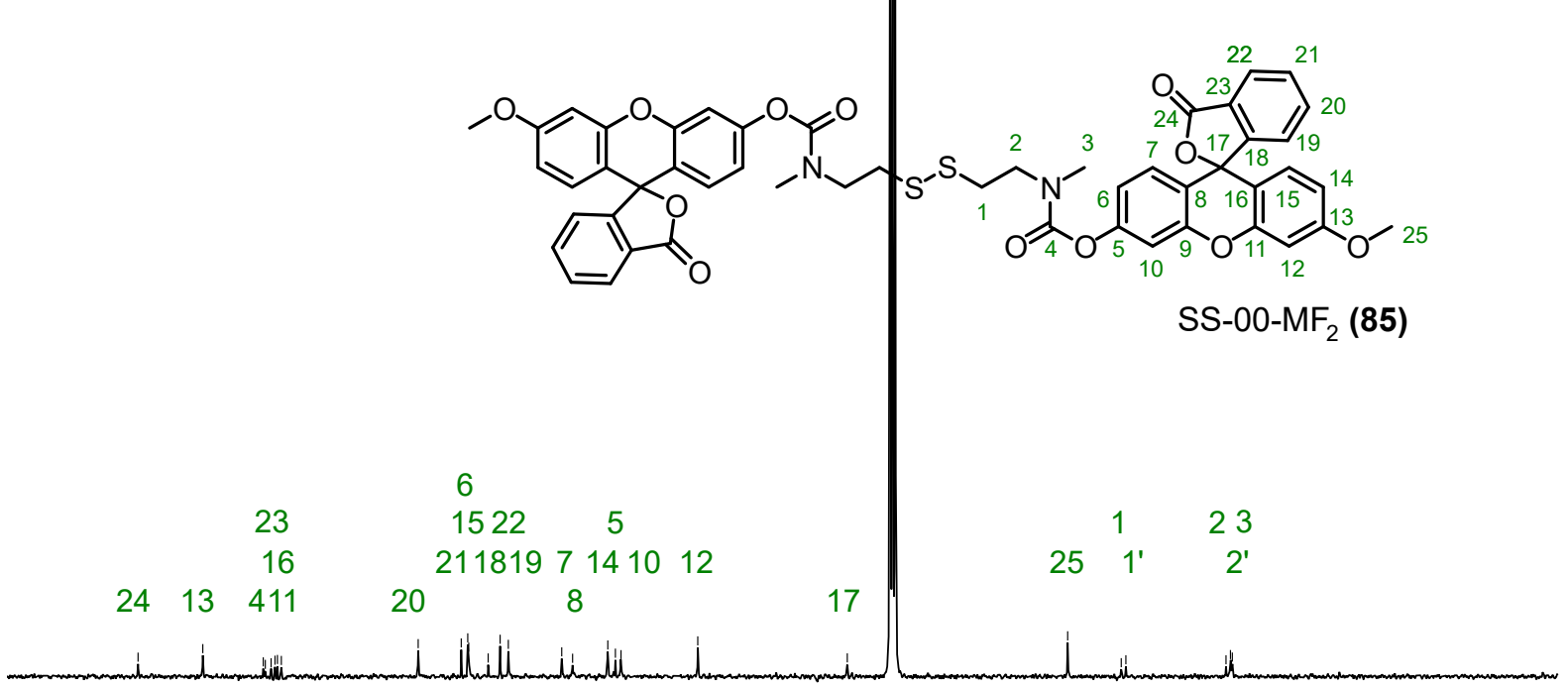

180

160

140

120

100

80

60

40

20

0

$\delta(\mathrm{ppm})$ 
cis-2',7'-difluoro-3'-isopropoxy-3-oxo-3H-spiro[isobenzofuran-1,9'-xanthen]-6'-yl-hexahydro$[1,2]$ dithiino[4,5-b]pyridine-1(2H)-carboxylate (SS66C-IG, 86)

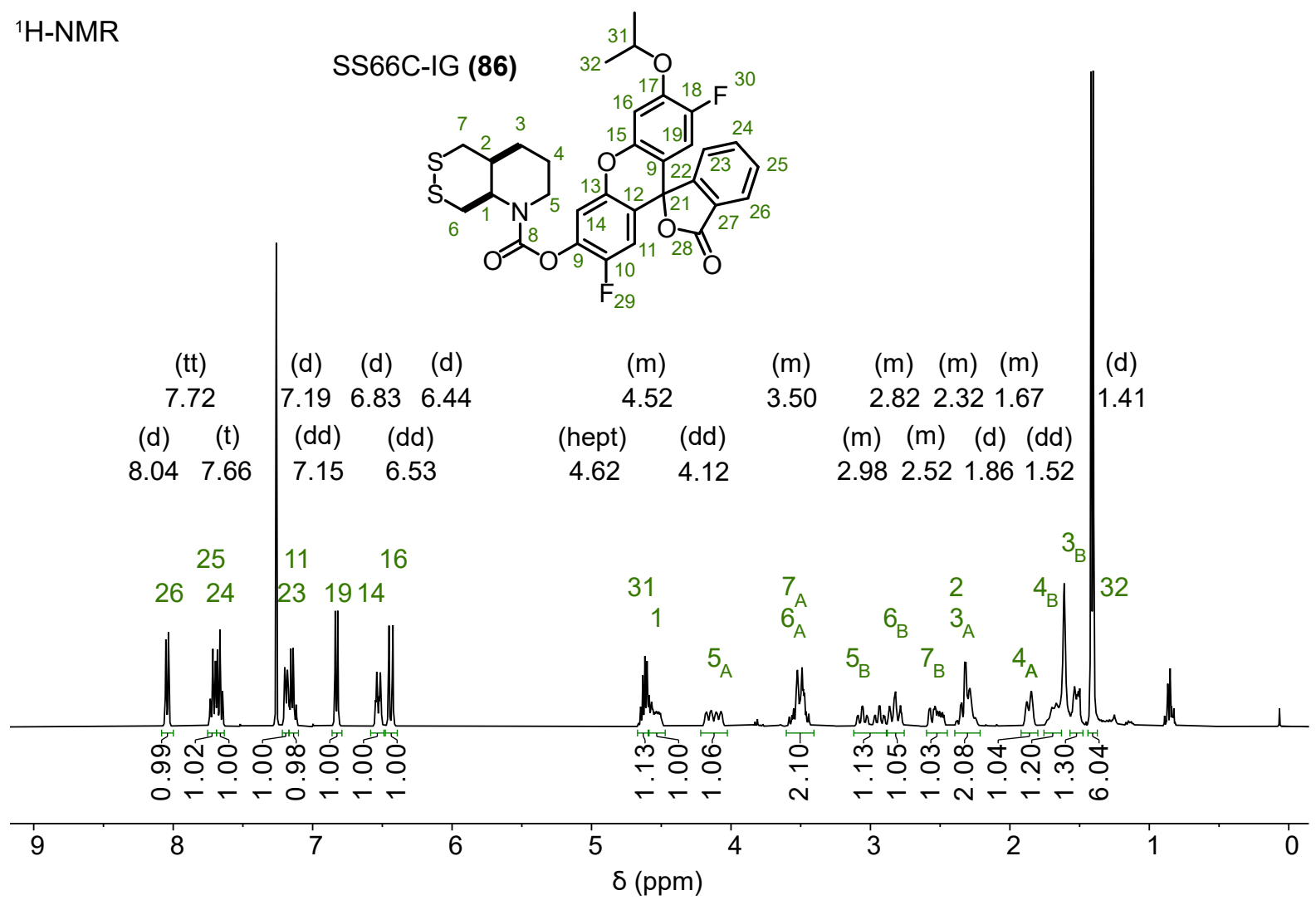

${ }^{13} \mathrm{C}-\mathrm{NMR}$

$\infty$ n

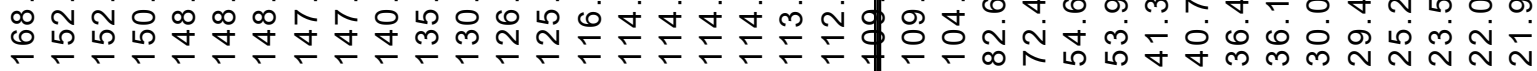<smiles>CC(C)Oc1cc2c(cc1F)C1(OC2=O)c2cc(F)c(OC(=O)N3CCCC4CSSCC43)cc2OC(=O)c2ccccc21</smiles>

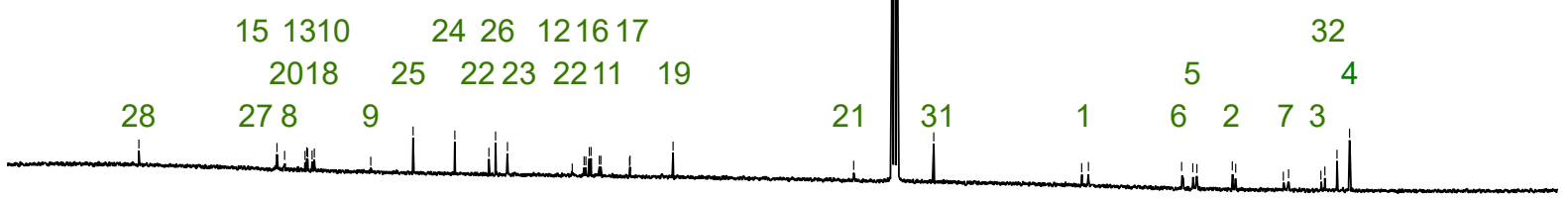

180

$160 \quad 140$

120

100

$8 \dot{0}$

60

40

20

$\delta(\mathrm{ppm})$ 
cis-2',7'-difluoro-3'-isopropoxy-3-oxo-3H-spiro[isobenzofuran-1,9'-xanthen]-6'-yl hexahydrofuro [3,4-b]pyridine-1(2H)-carboxylate (O56-IG, 87)

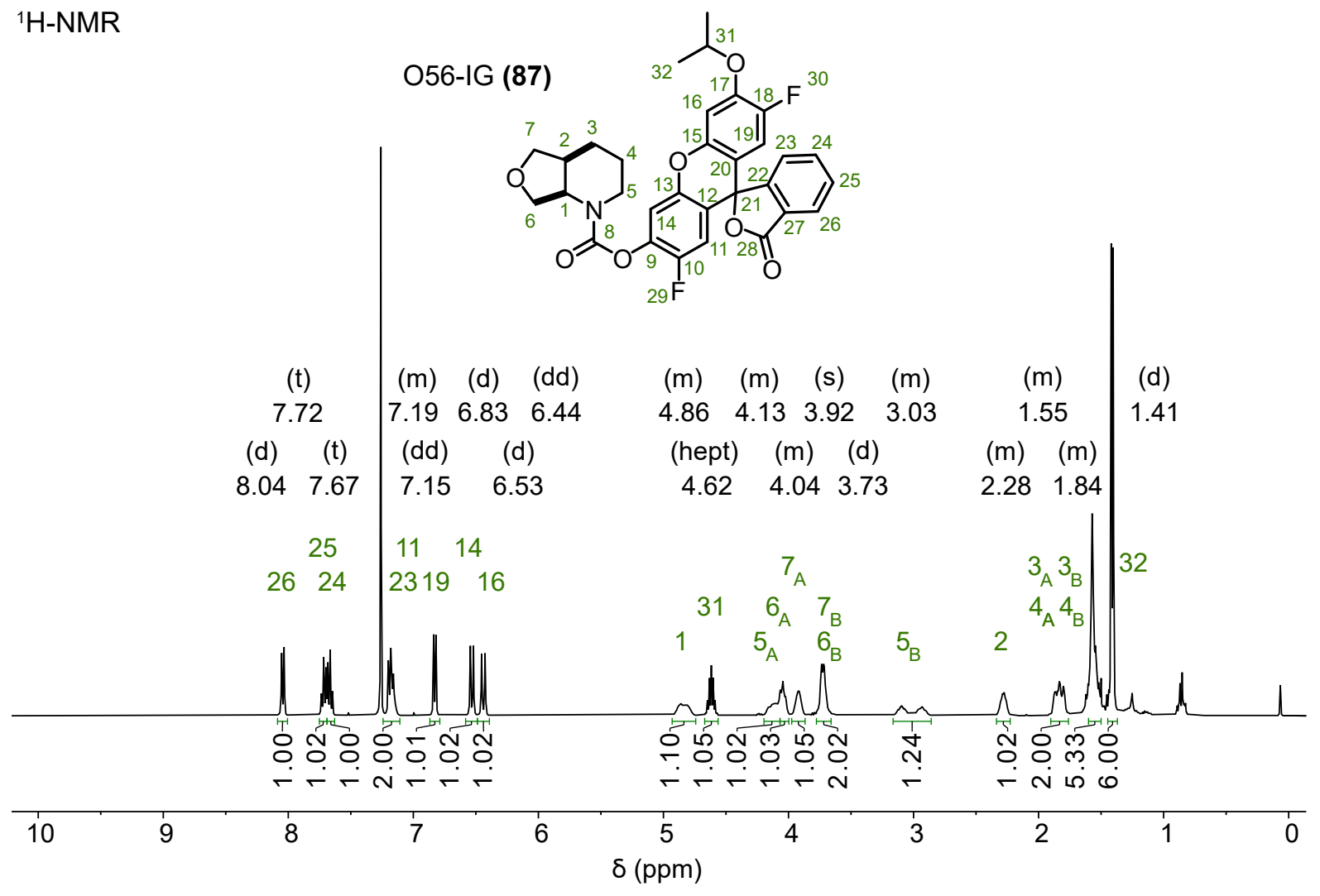

${ }^{13} \mathrm{C}-\mathrm{NMR}$

ம

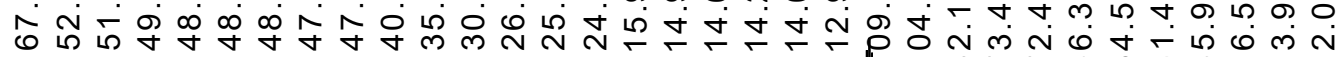
은
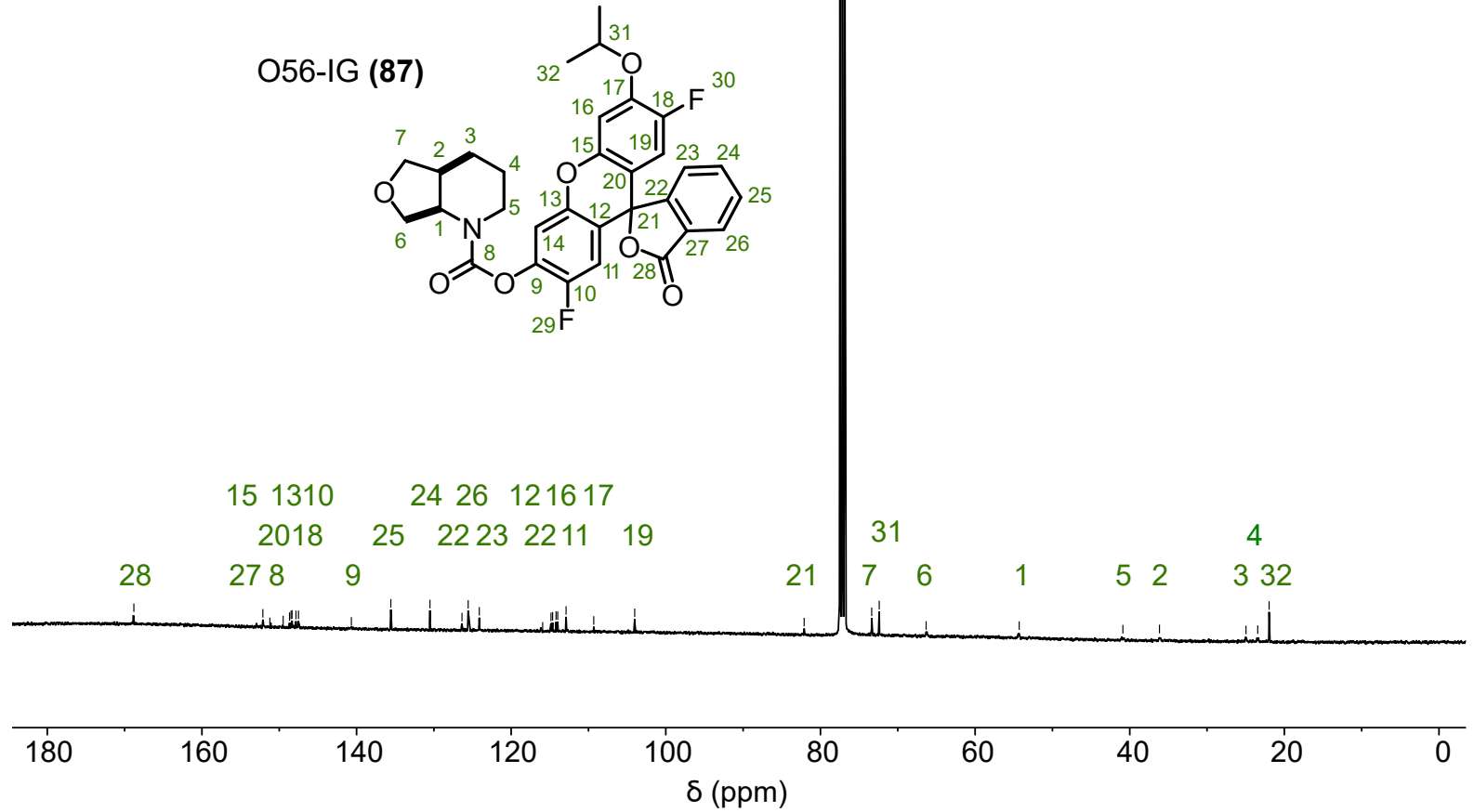
2',7'-difluoro-3'-isopropoxy-3-oxo-3H-spiro[isobenzofuran-1,9'-xanthen]-6'-yl ((S)-1,2-dithian-4yl)(methyl)carbamate (SS60-IG, 88)

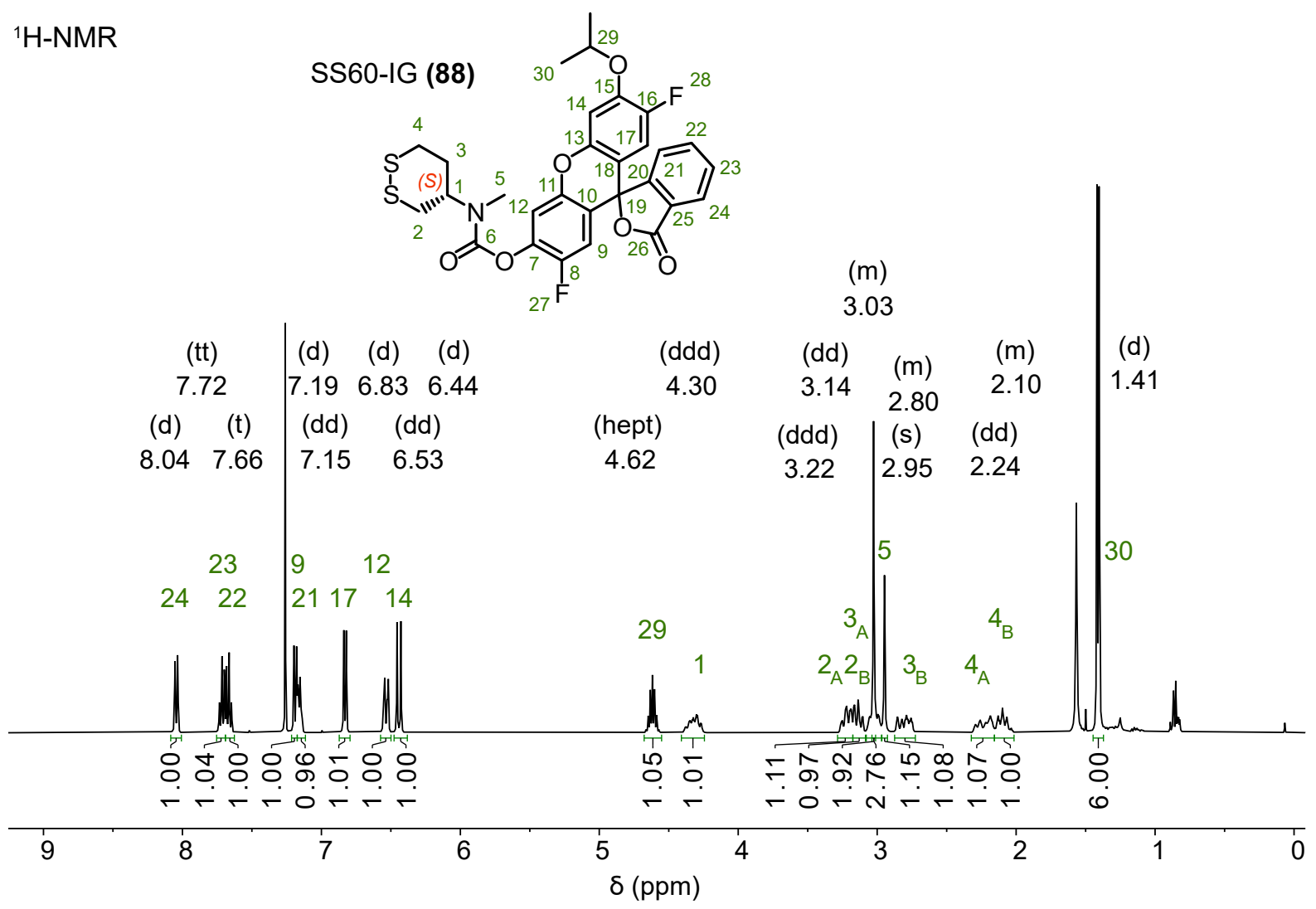

${ }^{13} \mathrm{C}-\mathrm{NMR}$

$\infty$ o 0 .

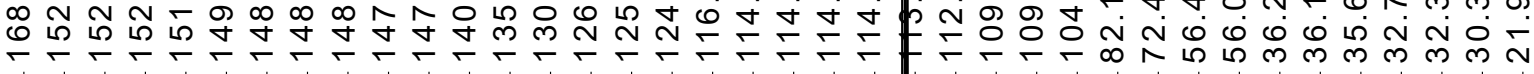

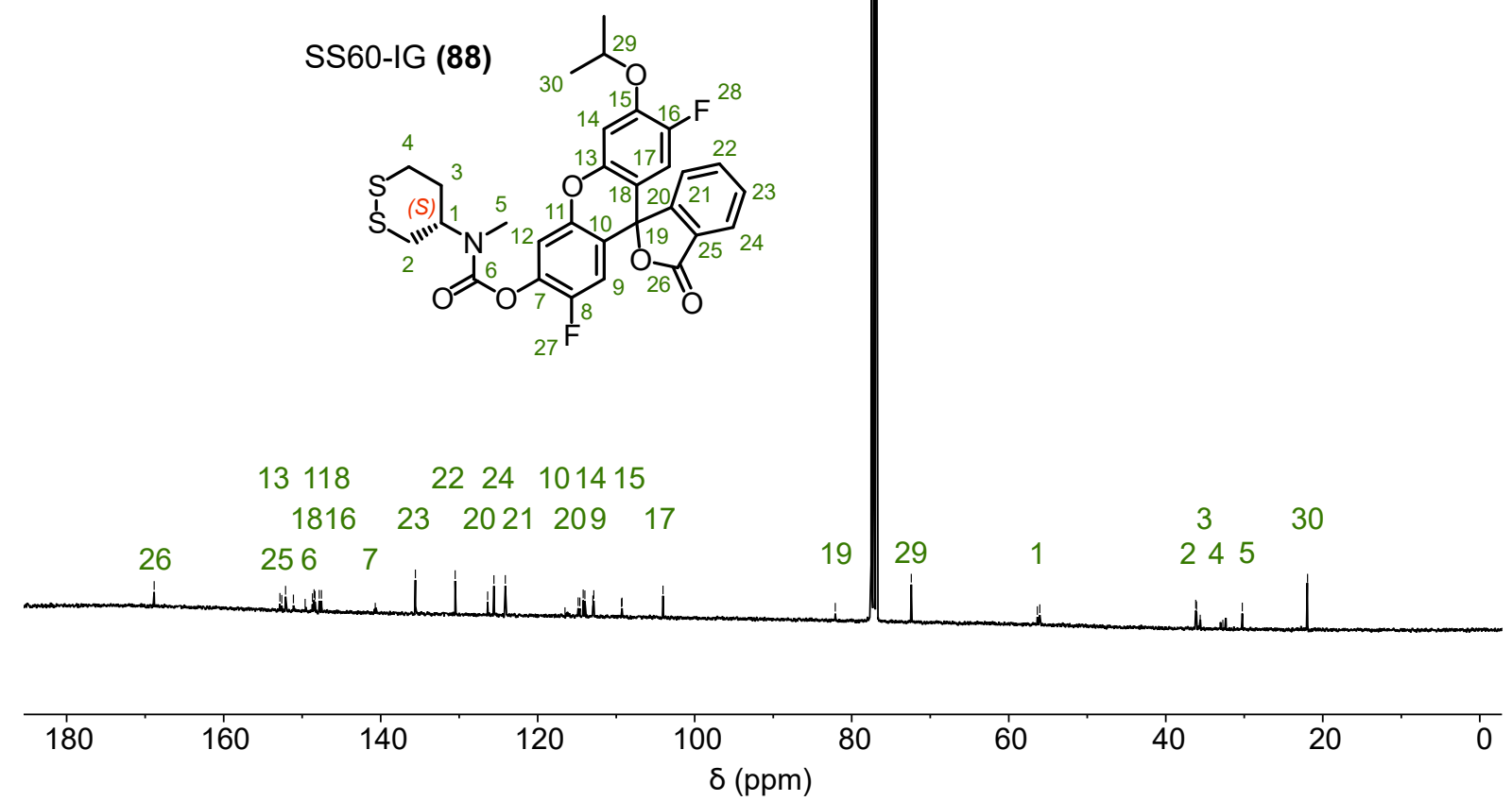


${ }^{1} \mathrm{H}-\mathrm{NMR}$

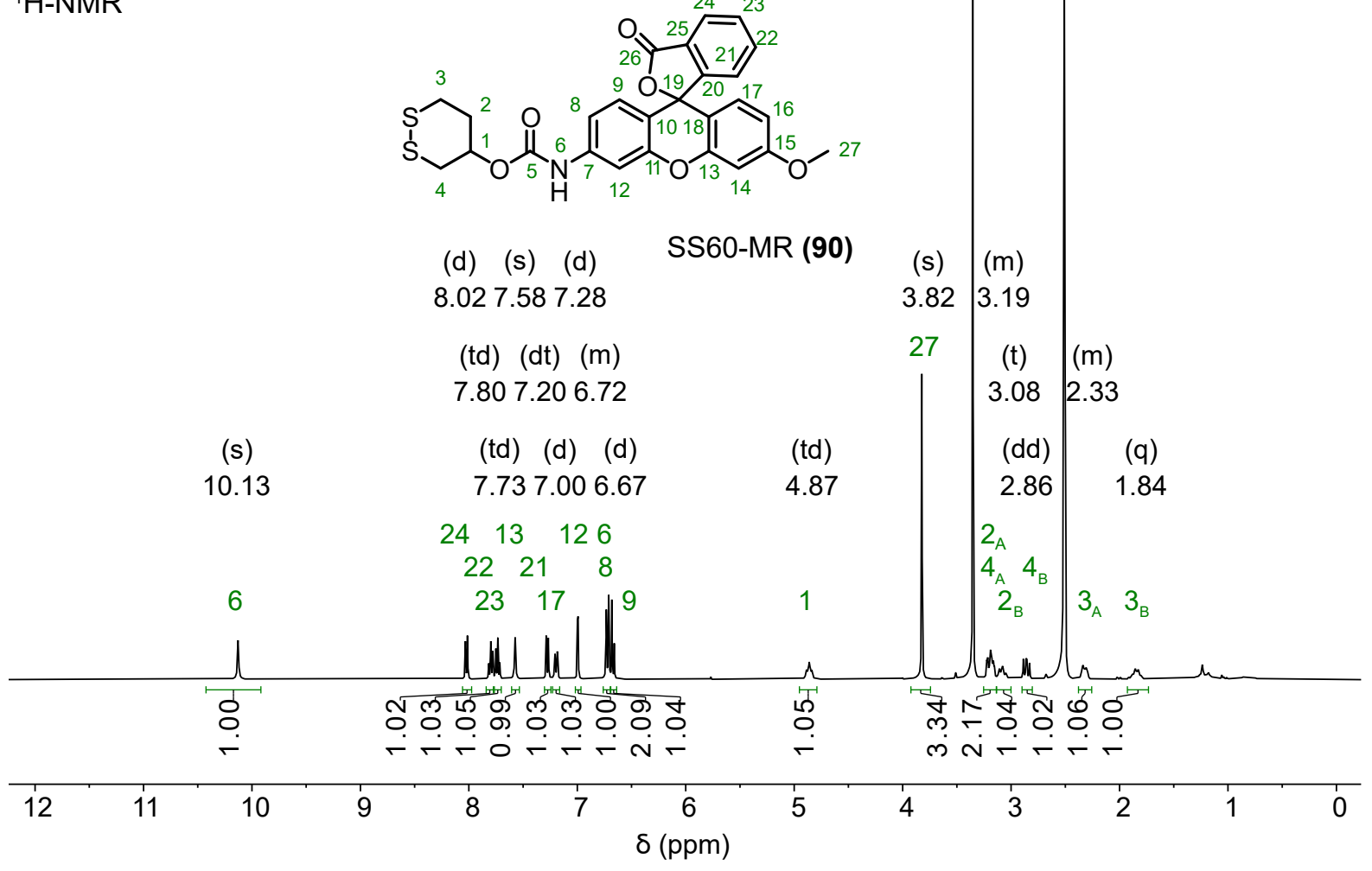

${ }^{13} \mathrm{C}-\mathrm{NMR}$

r.

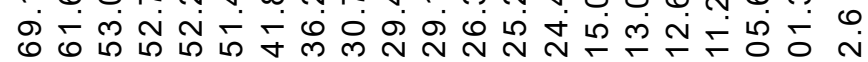

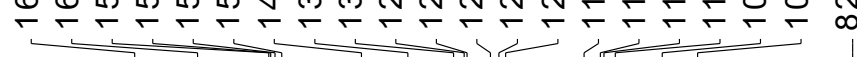

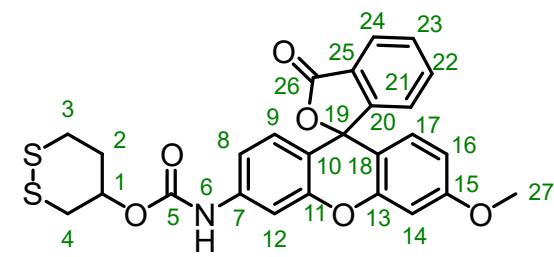

SS60-MR (90)

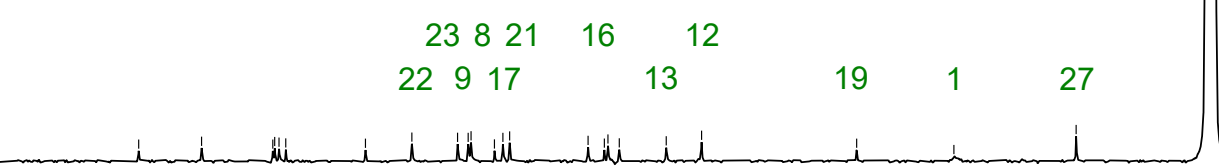

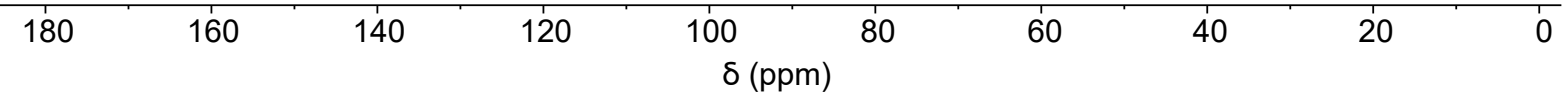




\section{X-ray crystallographic data}

trans-(hexahydro-[1,2]dithiino[4,5-b]pyridin-1(2H)-yl)benzamide (SS66T-Bz, 65)

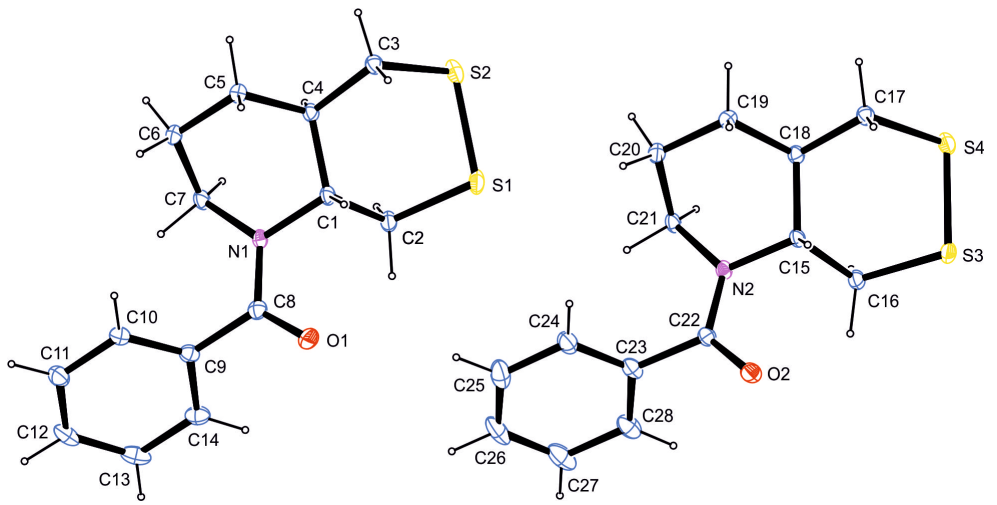

compound

65

CCDC number

2072442

net formula

$\mathrm{C}_{14} \mathrm{H}_{17} \mathrm{NOS}_{2}$

$M_{\mathrm{r}} / \mathrm{g} \mathrm{mol} \mathrm{m}^{-1}$

279.40

crystal size/mm

$0.080 \times 0.060 \times 0.040$

$T / \mathrm{K}$

radiation

102.(2)

MoKa

diffractometer

crystal system

'Bruker D8 Venture TXS'

space group

monoclinic
alA
$b / \AA ̊$
$c / \AA ̊$
$\alpha /{ }^{\circ}$
$\beta /{ }^{\circ}$
$Y /^{\circ}$
$V / \AA^{3}$

'P 1 c 1'

12.6211(6)

10.9095(5)

10.2423(5)

90

106.629(2)

90

1351.28(11)

$Z$

4

calc. density $/ \mathrm{g} \mathrm{cm}^{-3}$

1.373

0.381

Multi-Scan

absorption correction

$0.95-0.98$

transmission factor range

26059

$R_{\text {int }}$

0.0297

0.0252

mean $\sigma(I) / I$

$\theta$ range

observed refls.

3.369-27.101

5745

$x, y$ (weighting scheme)

hydrogen refinement

$0.0314,0.2724$

constr

Flack parameter

$0.036(18)$

refls in refinement

5929

325

parameters

2

$R\left(F_{0}\right)$

$R_{\mathrm{w}}\left(F^{2}\right)$

0.0614

$S$

1.056

0.001

shift/errormax

0.328

max electron density/e $\AA^{-3}$

$-0.230$ 


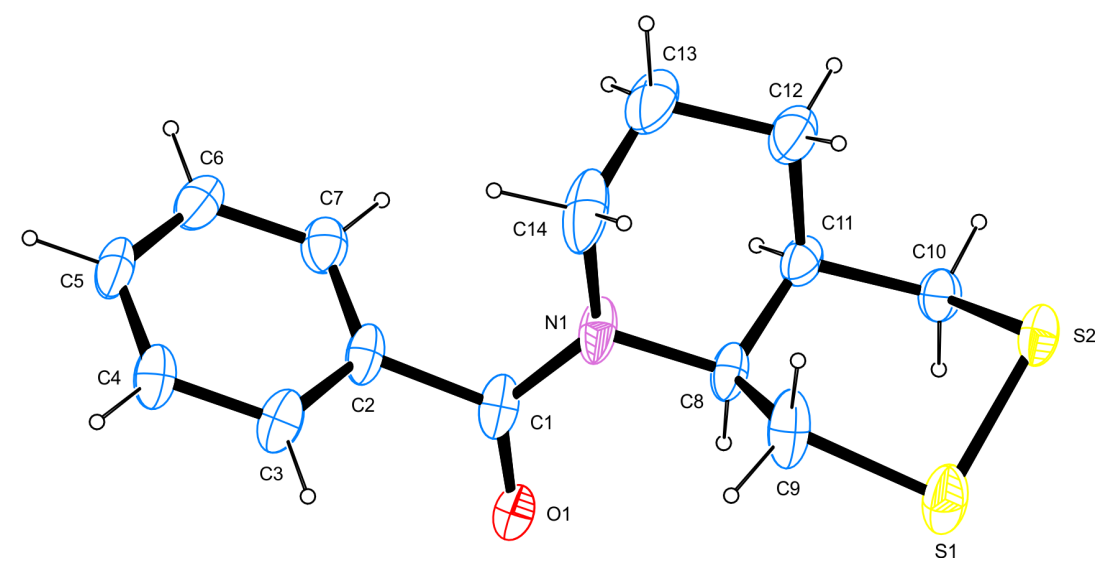

compound

CCDC number

net formula

$\mathrm{Mr}_{\mathrm{r}} / \mathrm{g} \mathrm{mol} \mathrm{m}^{-1}$

crystal size/mm

$T / \mathrm{K}$

radiation

diffractometer

crystal system

space group

alA

$b / \AA ̊$

$c / \AA ̊$

$\alpha /{ }^{\circ}$

$\beta /{ }^{\circ}$

$\mathrm{Y} /^{\circ}$

$V I \AA^{3}$

$Z$

calc. density $/ \mathrm{g} \mathrm{cm}^{-3}$

$\mu / \mathrm{mm}^{-1}$

absorption correction

transmission factor range

refls. measured

$R_{\text {int }}$

mean $\sigma(I) / I$

$\theta$ range

observed refls.

$x, y$ (weighting scheme)

hydrogen refinement

refls in refinement

parameters

restraints

$R\left(F_{\mathrm{obs}}\right)$

$R_{\mathrm{w}}\left(F^{2}\right)$

$S$

shift/errormax

max electron density/e $\AA^{-3}$

min electron density/e $\AA^{-3}$
66

2072441

$\mathrm{C}_{14} \mathrm{H}_{17} \mathrm{NOS}_{2}$

279.40

$0.080 \times 0.050 \times 0.030$

102.(2)

MoKa

'Bruker D8 Venture TXS'

monoclinic

'C $12 / \mathrm{c} 1$ '

18.5223(5)

6.4668(2)

24.1601(8)

90

109.8500(10)

90

2721.96(14)

8

1.364

0.378

Multi-Scan

0.92-0.99

20433

0.0360

0.0249

$3.360-26.730$

2543

$0.0236,7.1329$

constr

2865

163

18

0.0468

0.1059

1.179

0.001

0.336

$-0.354$

ISOR applied in ordert o improve the anisotropic displacement parameters of $\mathrm{C} 12, \mathrm{C} 13$ and $\mathrm{C} 14$. 
(S)-N-methyl-N-(1,2-dithian-4-yl)benzamide (SS60-Bz, 67)

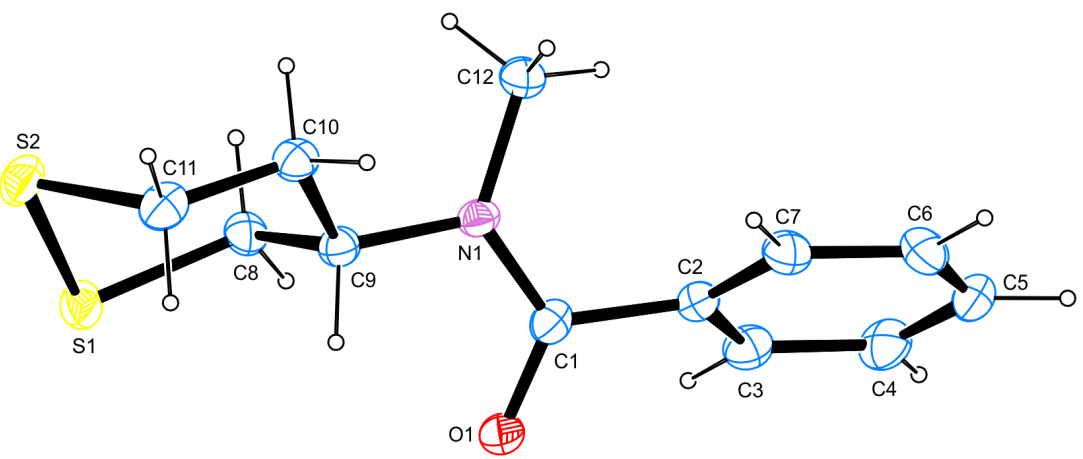

compound

CCDC number

net formula

$\mathrm{Mr}_{\mathrm{r}} / \mathrm{g} \mathrm{mol}{ }^{-1}$

crystal size/mm

$T / \mathrm{K}$

radiation

diffractometer

crystal system

space group

alA

$b / \AA$

$c / \AA$

$\alpha /{ }^{\circ}$

$\beta /^{\circ}$

$Y /^{\circ}$

$V / \AA^{3}$

Z

calc. density $/ \mathrm{g} \mathrm{cm}^{-3}$

$\mu / \mathrm{mm}^{-1}$

absorption correction

transmission factor range

refls. measured

$R_{\text {int }}$

mean $\sigma(I) / I$

$\theta$ range

observed refls.

$x, y$ (weighting scheme)

hydrogen refinement

Flack parameter

refls in refinement

parameters

restraints

$R\left(F_{\text {obs }}\right)$

$R_{\mathrm{w}}\left(F^{2}\right)$

$S$

shift/errormax

max electron density/e $\AA^{-3}$

min electron density/e $\AA^{-3}$
67

2072438

$\mathrm{C}_{12} \mathrm{H}_{15} \mathrm{NOS}_{2}$

253.37

$0.100 \times 0.100 \times 0.090$

107.(2)

MoKa

'Bruker D8 Venture TXS'

triclinic

'P 1'

5.4422(8)

5.9216(9)

10.5764(15)

88.173(5)

79.770(5)

65.997(4)

306.11(8)

1

1.374

0.413

Multi-Scan

0.85-0.96

6796

0.0356

0.0398

3.770-27.099

2479

$0,0.4159$

constr

0.09(14)

2538

147

3

0.0399

0.0949

1.150

0.001

0.442

$-0.336$

Refined as inversion twin, BASF 0.09. 


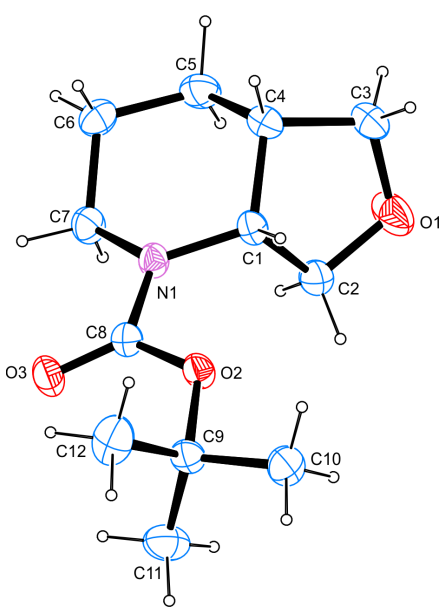

compound

5

CCDC number

2072440

net formula

$\mathrm{Mr}_{\mathrm{r}} / \mathrm{g} \mathrm{mol}{ }^{-1}$

$\mathrm{C}_{12} \mathrm{H}_{21} \mathrm{NO}_{3}$

227.30

crystal size $/ \mathrm{mm}$

$0.100 \times 0.060 \times 0.050$

$T / \mathrm{K}$

radiation

102.(2)

MoKa

diffractometer

'Bruker D8 Venture TXS'

crystal system

monoclinic

space group

'P $121 / \mathrm{c} 1$ '

al§

$b / \AA$

8.8168(4)

$17.0374(7)$

$c / \AA ̊$

$8.4751(3)$

$\alpha /{ }^{\circ}$

90

$\beta /{ }^{\circ}$

$Y /^{\circ}$

98.0550(10)

90

$Z$

1260.53(9)

4

calc. density $/ \mathrm{g} \mathrm{cm}^{-3}$

1.198

$\mu / \mathrm{mm}^{-1}$

0.085

Multi-Scan

absorption correction

0.96-1.00

transmission factor range

21814

0.0394

$R_{\text {int }}$

0.0230

mean $\sigma(I) / I$

2.706-27.097

2396

observed refls.

$x, y$ (weighting scheme)

$0.0358,0.5700$

hydrogen refinement

constr

refls in refinement

2772

parameters

148

restraints

0

$R\left(F_{\text {obs }}\right)$

0.0395

$R_{\mathrm{w}}\left(F^{2}\right)$

0.1024

1.060

shift/error ${ }_{\text {max }}$

0.001

0.254

max electron density/e $\AA^{-3}$

$-0.203$

min electron density/e $\AA^{-3}$

pace group. 


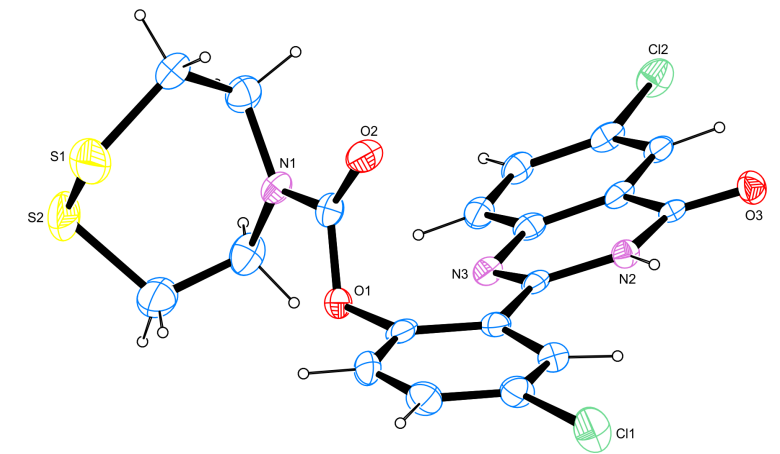

compound

CCDC number

net formula

$\mathrm{Mr}_{\mathrm{r}} / \mathrm{g} \mathrm{mol}{ }^{-1}$

crystal size/mm

$T / \mathrm{K}$

radiation

diffractometer

crystal system

space group

alA

b/Å

$c / \AA$

$\alpha /{ }^{\circ}$

$\beta /{ }^{\circ}$

$\mathrm{Y} /{ }^{\circ}$

$V / \AA^{3}$

$Z$

calc. density $/ \mathrm{g} \mathrm{cm}^{-3}$

$\mu / \mathrm{mm}^{-1}$

absorption correction

transmission factor range

refls. measured

$R_{\text {int }}$

mean $\sigma(I) / I$

$\theta$ range

observed refls.

$x, y$ (weighting scheme)

hydrogen refinement

refls in refinement

parameters

restraints

$R\left(F_{\text {obs }}\right)$

$R_{\mathrm{w}}\left(F^{2}\right)$

$S$

shift/errormax

max electron density/e $\AA^{-3}$

min electron density/e $\AA^{-3}$
75

2072439

$\mathrm{C}_{19} \mathrm{H}_{15} \mathrm{Cl}_{2} \mathrm{~N}_{3} \mathrm{O}_{3} \mathrm{~S}_{2}$

468.36

$0.100 \times 0.060 \times 0.020$

102.(2)

MoKa

'Bruker D8 Venture TXS'

monoclinic

'P $121 / \mathrm{c} 1$ '

18.2066(9)

6.1995(3)

18.0583(10)

90

102.270(2)

90

1991.71(18)

4

1.562

0.563

Multi-Scan

0.84-0.99

3611

0.0763

0.0366

$2.290-25.346$

3374

$0,4.3669$

constr

3611

272

0

0.0492

0.0992

1.172

0.001

0.283

$-0.329$

Refined as a 2-component twin. BASF 0.35. C2 along (100). S2 moiety disordered, split model applied, sof ratio refined to $0.95 / 0.05$, minor part refined isotropically. Minor part not depicted in figure above. 


\section{Supporting Information Bibliography}

(1) Hong, K.-H.; Kim, D. I.; Kwon, H.; Kim, H.-J. A Fluoresceinylcarbonate-Based Fluorescent Probe for the Sensitive Detection of Biothiols in a HEPES Buffer and Its Cellular Expression. RSC Advances 2014, 4 (2), 978-982. https://doi.org/10.1039/C3RA42935K.

(2) Lim, C. S.; Masanta, G.; Kim, H. J.; Han, J. H.; Kim, H. M.; Cho, B. R. Ratiometric Detection of Mitochondrial Thiols with a Two-Photon Fluorescent Probe. Journal of the American Chemical Society 2011, 133 (29), 11132-11135. https://doi.org/10.1021/ja205081s.

(3) Vrudhula, V. M.; MacMaster, J. F.; Li, Z.; Kerr, D. E.; Senter, P. D. Reductively Activated Disulfide Prodrugs of Paclitaxel. Bioorganic \& Medicinal Chemistry Letters 2002, 12, 3591-3594.

(4) Henne, W. A.; Doorneweerd, D. D.; Hilgenbrink, A. R.; Kularatne, S. A.; Low, P. S. Synthesis and Activity of a Folate Peptide Camptothecin Prodrug. Bioorganic \& Medicinal Chemistry Letters 2006, 16 (20), 5350-5355. https://doi.org/10.1016/j.bmcl.2006.07.076.

(5) Zhang, L.; Duan, D.; Liu, Y.; Ge, C.; Cui, X.; Sun, J.; Fang, J. Highly Selective Off-On Fluorescent Probe for Imaging Thioredoxin Reductase in Living Cells. Journal of the American Chemical Society 2014, 136 (1), 226-233. https://doi.org/10.1021/ja408792k.

(6) Kong, F.; Zhao, Y.; Liang, Z.; Liu, X.; Pan, X.; Luan, D.; Xu, K.; Tang, B. Highly Selective Fluorescent Probe for Imaging $\mathrm{H}_{2}$ Se in Living Cells and in Vivo Based on the Disulfide Bond. Analytical Chemistry 2017, 89 (1), 688-693. https://doi.org/10.1021/acs. analchem.6b03136.

(7) Lee, S. H.; Kohn, H. 7-N,7'-N'-(1",2"-Dithianyl-3",6"-Dimethylenyl)Bismitomycin C: Synthesis and Nucleophilic Activation of a Dimeric Mitomycin. Organic \& Biomolecular Chemistry 2005, 3 (3), 471-482. https://doi.org/10.1039/B414806A.

(8) Ziv, I. Pro-Drugs and Related Methods. WO2017017669A1, 2017.

(9) Li, X.; Hou, Y.; Meng, X.; Ge, C.; Ma, H.; Li, J.; Fang, J. Selective Activation of a Prodrug by Thioredoxin Reductase Providing a Strategy to Target Cancer Cells. Angewandte Chemie International Edition 2018, 57 (21), 6141-6145. https://doi.org/10.1002/anie.201801058.

(10) Zeisel, L.; Felber, J. G.; Poczka, L.; Scholzen, K.; Maier, M. S.; Arnér, E. S. J.; Thorn-Seshold, J.; Thorn-Seshold, O. Selective Cellular Probes for Mammalian Thioredoxin Reductase TrxR1: Rational Design of Modular 1,2-Thiaselenane-Based "RX1" Redox Probes. paper in submission 2021.

(11) Felber, J. G.; Poczka, L.; Busker, S.; Theisen, U.; Zeisel, L.; Maier, M. S.; Brandstädter, C.; Scholzen, K.; Becker, K.; Arnér, E. S. J.; Ahlfeld, J.; Thorn-Seshold, O. Cyclic 5-Membered Disulfides Are Not Selective Substrates of Thioredoxin Reductase, but Are Opened Nonspecifically by Thiols. ChemRxiv 2020. https://doi.org/10.26434/chemrxiv.13483155.v1.

(12) Laurent, Q.; Sakai, N.; Matile, S. The Opening of 1,2-Dithiolanes and 1,2-Diselenolanes: Regioselectivity, Rearrangements, and Consequences for Poly(Disulfide)s, Cellular Uptake and Pyruvate Dehydrogenase Complexes. Helvetica Chimica Acta 2019, 102 (2), e1800209. https://doi.org/10.1002/hlca.201800209.

(13) Houk, J.; Whitesides, G. M. Structure-Reactivity Relations for Thiol-Disulfide Interchange. Journal of the American Chemical Society 1987, 109 (22), 6825-6836. https://doi.org/10.1021/ja00256a040.

(14) Gromer, S.; Arscott, L. D.; Williams, C. H.; Schirmer, R. H.; Becker, K. Human Placenta Thioredoxin Reductase: Isolation of the Selenoenzmye, Steady State Kinetics, and Inhibition by Therapeutic Gold Compounds. Journal of Biological Chemistry 1998, 273 (32), 20096-20101. https://doi.org/10.1074/jbc.273.32.20096.

(15) Urig, S.; Lieske, J.; Fritz-Wolf, K.; Irmler, A.; Becker, K. Truncated Mutants of Human Thioredoxin Reductase 1 Do Not Exhibit Glutathione Reductase Activity. FEBS Letters 2006, 580 (15), 3595-3600. https://doi.org/10.1016/j.febslet.2006.05.038.

(16) Nordhoff, A.; Buecheler, U. S.; Werner, D.; Schirmer, R. H. Folding of the Four Domains and Dimerization Are Impaired by the Gly446Glu Exchange in Human Glutathione Reductase. 
Implications for the Design of Antiparasitic Drugs. Biochemistry 1993, 32 (15), 4060-4066. https://doi.org/10.1021/bi00066a029.

(17) Sheldrick, G. M. SHELXT - Integrated Space-Group and Crystal-Structure Determination. Acta Crystallographica Section A Foundations and Advances 2015, 71 (1), 3-8. https://doi.org/10.1107/S2053273314026370.

(18) Farrugia, L. J. WinGX and ORTEP for Windows: An Update. Journal of Applied Crystallography 2012, 45 (4), 849-854. https://doi.org/10.1107/S0021889812029111.

(19) Liéby-Muller, F.; Marion, F.; Schmitt, P.; Annereau, J.-P.; Kruczynski, A.; Guilbaud, N.; Bailly, C. Synthesis and Biological Evaluation of (-)-6-O-Desmethylcryptopleurine and Analogs. Bioorganic \& Medicinal Chemistry Letters 2015, 25 (2), 184-187. https://doi.org/10.1016/j.bmcl.2014.11.086.

(20) Sánchez-Sancho, F.; Herradón, B. Short Syntheses of (S)-Pipecolic Acid, (R)-Coniine, and (S)$\delta$-Coniceine Using Biocatalytically-Generated Chiral Building Blocks. Tetrahedron: Asymmetry 1998, 9 (11), 1951-1965. https://doi.org/10.1016/S0957-4166(98)00178-5.

(21) Niphakis, M. J.; Turunen, B. J.; Georg, G. I. Synthesis of 6- and 7-Membered Cyclic Enaminones: Scope and Mechanism. The Journal of Organic Chemistry 2010, 75 (20), 6793-6805. https://doi.org/10.1021/jo100907u.

(22) Lukesh, J. C.; Palte, M. J.; Raines, R. T. A Potent, Versatile Disulfide-Reducing Agent from Aspartic Acid. Journal of the American Chemical Society 2012, 134 (9), 4057-4059. https://doi.org/10.1021/ja211931f.

(23) Moss, N.; Beaulieu, P.; Duceppe, J.-S.; Ferland, J.-M.; Gauthier, J.; Ghiro, E.; Goulet, S.; Guse, I.; Llinàs-Brunet, M.; Plante, R.; Plamondon, L.; Wernic, D.; Déziel, R. Ureido-Based Peptidomimetic Inhibitors of Herpes Simplex Virus Ribonucleotide Reductase: An Investigation of Inhibitor Bioactive Conformation. Journal of Medicinal Chemistry 1996, 39 (11), 2178-2187. https://doi.org/10.1021/jm950825x.

(24) Baugh, S. D. P.; Yang, Z.; Leung, D. K.; Wilson, D. M.; Breslow, R. Cyclodextrin Dimers as Cleavable Carriers of Photodynamic Sensitizers. Journal of the American Chemical Society 2001, 123 (50), 12488-12494. https://doi.org/10.1021/ja011709o.

(25) Butler, D.; Iwamoto, N.; Meena, M.; Svrzikapa, N.; Verdine, G. L.; Zlatev, I. Chiral Control. WO 2014/012081 A2.

(26) Batisse, C.; Dransart, E.; Ait Sarkouh, R.; Brulle, L.; Bai, S.-K.; Godefroy, S.; Johannes, L.; Schmidt, F. A New Delivery System for Auristatin in STxB-Drug Conjugate Therapy. European Journal of Medicinal Chemistry 2015, 95, 483-491. https://doi.org/10.1016/j.ejmech.2015.03.047.

(27) Fulmer, G. R.; Miller, A. J. M.; Sherden, N. H.; Gottlieb, H. E.; Nudelman, A.; Stoltz, B. M.; Bercaw, J. E.; Goldberg, K. I. NMR Chemical Shifts of Trace Impurities: Common Laboratory Solvents, Organics, and Gases in Deuterated Solvents Relevant to the Organometallic Chemist. Organometallics 2010, 29 (9), 2176-2179. https://doi.org/10.1021/om100106e.

(28) Na, Y.; Kohn, H. Quinone-Cyclized Porfiromycins. Heterocycles 2001, 55 (7), 1347-1364. https://doi.org/10.3987/COM-01-9215.

(29) Ollivier, N.; Dheur, J.; Mhidia, R.; Blanpain, A.; Melnyk, O. Bis(2-Sulfanylethyl)Amino Native Peptide Ligation. Organic Letters 2010, 12 (22), 5238-5241. https://doi.org/10.1021/ol102273u.

(30) Li, X.; Zhang, B.; Yan, C.; Li, J.; Wang, S.; Wei, X.; Jiang, X.; Zhou, P.; Fang, J. A Fast and Specific Fluorescent Probe for Thioredoxin Reductase That Works via Disulphide Bond Cleavage. Nature Communications 2019, 10 (2745), 1-12. https://doi.org/10.1038/s41467-01910807-8.

(31) Aw, J.; Shao, Q.; Yang, Y.; Jiang, T.; Ang, C.; Xing, B. Synthesis and Characterization of 2-(2'Hydroxy-5'-Chlorophenyl)-6-Chloro-4(3 $H$ )-Quinazolinone-Based Fluorogenic Probes for Cellular Imaging of Monoamine Oxidases. Chemistry - An Asian Journal 2008, NA-NA. https://doi.org/10.1002/asia.201000025. 
(32) More, K. N.; Lim, T.-H.; Kim, S.-Y.; Kang, J.; Inn, K.-S.; Chang, D.-J. Characteristics of New Bioreductive Fluorescent Probes Based on the Xanthene Fluorophore: Detection of Nitroreductase and Imaging of Hypoxic Cells. Dyes and Pigments 2018, 151, 245-253. https://doi.org/10.1016/j.dyepig.2018.01.008.

(33) Fu, Q.; Li, H.; Duan, D.; Wang, C.; Shen, S.; Ma, H.; Liu, Z. External-Radiation-Induced Local Hydroxylation Enables Remote Release of Functional Molecules in Tumors. Angewandte Chemie International Edition https://doi.org/10.1002/anie.202005612.

2020, 59

(48), 21546-21552. 